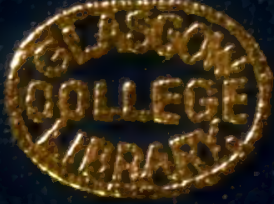




\section{STORE}

\section{Glasgow \\ Uníversity Tibrary}

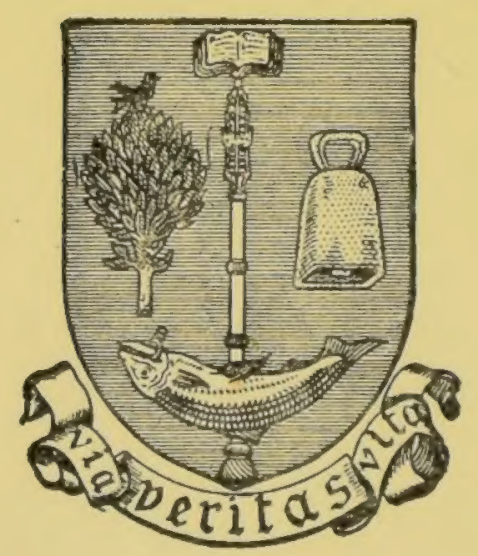

Store

HA11641

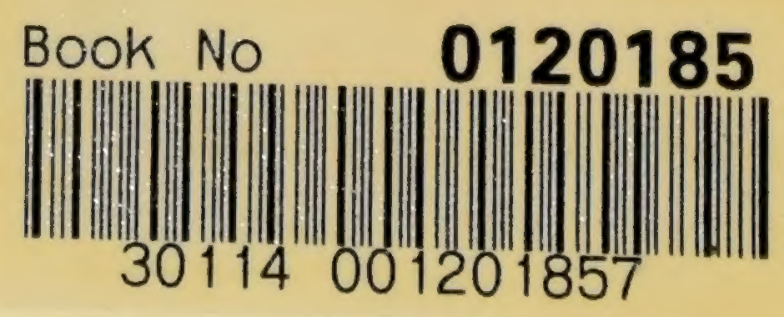


Glasgow University Library
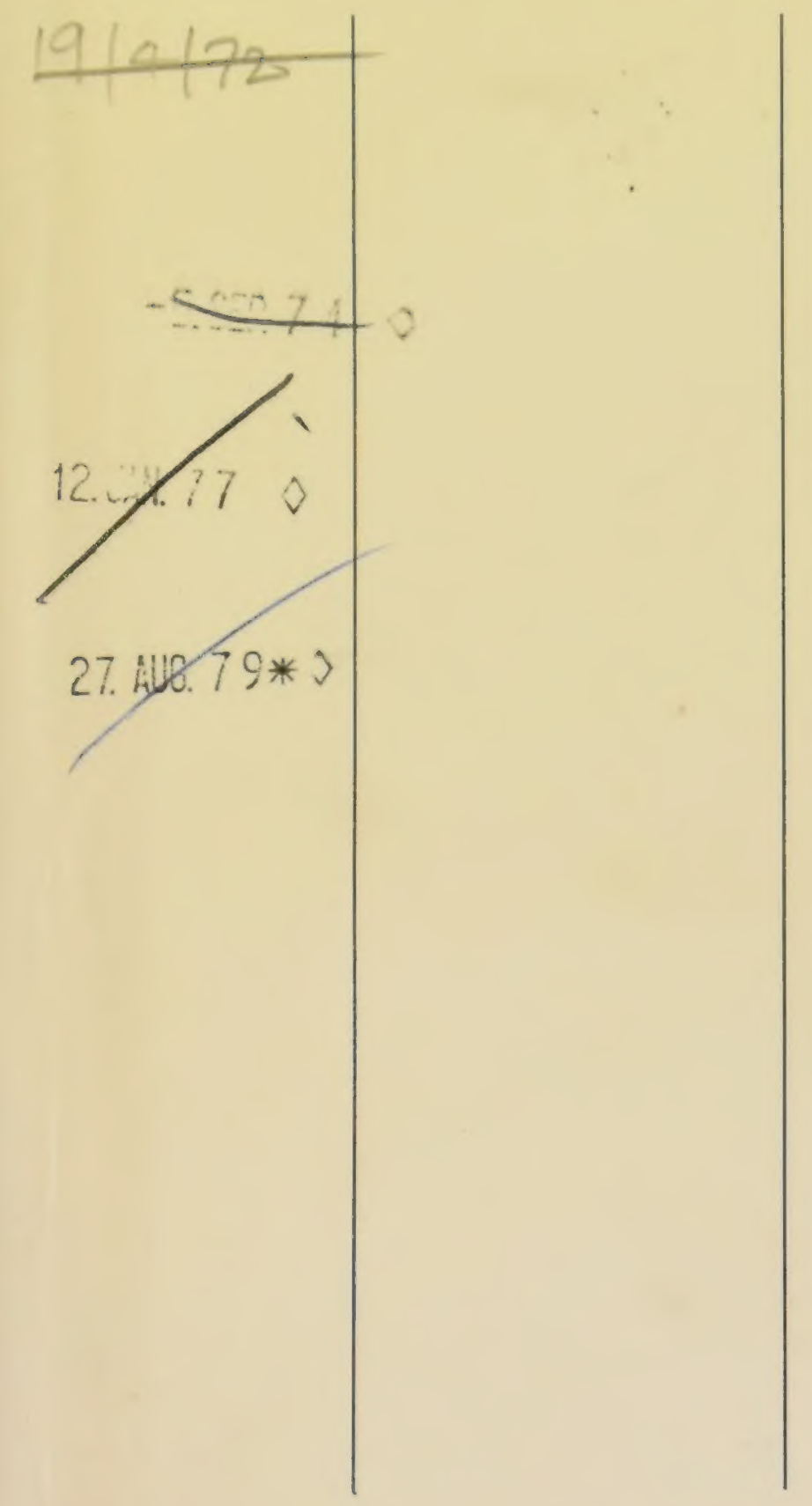

GUL 68.18 

Digitized by the Internet Archive in 2015

https://archive.org/details/b21470303 



\section{PART THIRD}

OF A

\section{S2RIมS}

OF

\section{ELEMENTARY LECTURES}

ON THE

\section{VETERINARY ART:}

WHEREIN THE

ANATOMY, PHYSIOLOGY, AND PATHOLOGY

OF THE

\section{HORSE,}

A R B

ESSAYED ON THE GENERAL PRINCIPLES

OF

\section{Stitedical Extemce.}

BY

\section{WILLIAM PERCIVALL,}

Member of the Royal College of Surgeons in London; Licentiate of the Society of Apothecaries; and late Veterinary Surgeon of the

$$
\text { Royal Artillery. }
$$

"Qui secat, sit anatomes peritus, quia sub hoc medico et artifice, omnia tutissime et felicisaime peraguntur,"-Fabricius ab Aquapendente.

"Morborum quoque te causas et signa docebo."-Virgil.

\section{LONDON:}

Published by

LONGMAN, REES, ORME, BROWN, AND GREEN, Paternoster Row. 


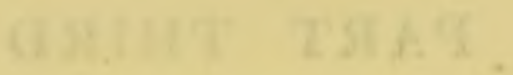

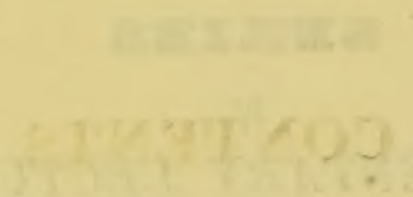

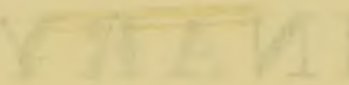

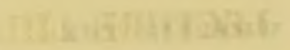

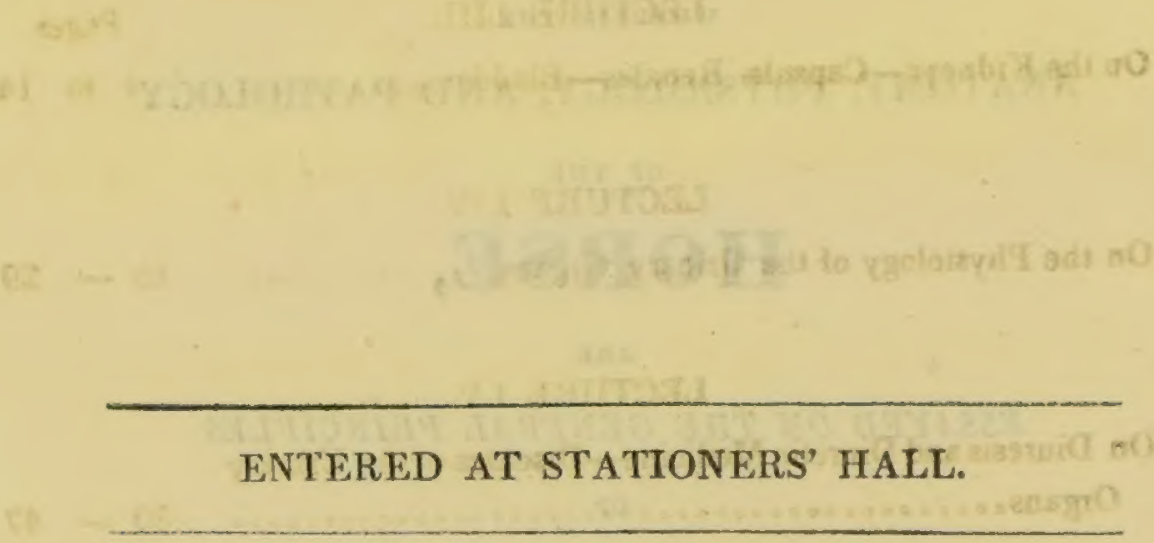

ENTERED AT STATIONERS' HALL.

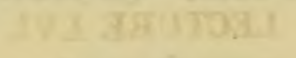

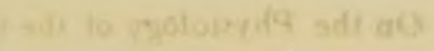$$
922-k .2
$$ 


\section{CONTENTS.}

LECTURE LIII.
On the Kidneys-Capsulæ Renales-Bladder................. 1 to 14

LECTURE LIV.

On the Physiology of the Urinary Organs ........... $15-29$

LECTURE LV.

On Diuresis and Diuretic Medicine-Diseases of the Urinary

Organs............................... $30-47$

\section{LECTURE LVI.}

On the Testes and Vesiculæ Seminales

$48-63$

LECTURE LVII.

On the Penis and Prostrate Glands .............. $64-76$

LECTURE LVIII.

On the Female Organs of Generation ............. $77-93$

LECTURE LIX.

On the Physiology of the Organs of Generation, Male and

Female .............................. $94-113$

LECTURE LX.

On Castration............................. 114 - 131 
vi

CONTENTS.

LECTURE LXI.

Pages

On the Appendages of the Eye............... 132 to 153

LECTURE LXII.

On the Eyeball $154-179$

LECTURE LXIII.

On Vision $180-202$

LECTURE LXIV.

On the Diseases of the Eye $203-236$

\section{LECTURE LXV.}

On the Dissection of the Brain and Spinal Marrow ..... 237 - 259

\section{LECTURE LXVI.}

On the Origin and Distribution of the Nerves

$260-276$

LECTURE LXVII.

The Subject continued $277-288$

LECTURE LXVIII.

The Subject continued $289-304$

LECTURE LXIX.

On the Distribution of the Arteries $305-321$

\section{LECTURE LXX.}

The Subject continued $322-335$

\section{LECTURE LXXI.}

The Subject continued 
CONTENTS.

vii

LECTURE LXXI.

Pages

On the Distribution of the Veins ............... 354 to 372

\section{LECTURE LXXIII.}

On the Distribution of the Absorbents........... $373-386$

LECTURE LXXIV.

On Fever

$387-405$

LECTURE LXXV.

On Mange and other Cutaneous Affections-Grease ..... 406 - 428

LECTURE LXXVI.

On Staggers-Strangles..................... $429-445$

LECTURE LXXVII.

On Glanders and Farcy (Professor Coleman's Opinions) .. $446-464$

LECTURE LXXVIII.

The Subject continued (Professor Dupuy's Opinions) ... $465-502$ 



\title{
VETERINARY LECTURES.
}

\section{LECTURE LIII.}

\begin{abstract}
Having concluded my observations on the chylopoietic viscera, I shall take up the consideration of those employed in the secretion and excretion of urine, the URINARY ORGANS as they are generally called, beginning with
\end{abstract}

\section{The Kidneys.}

THE KIDNEYS are two reddish ovoid bodies, situated in the lumbar regions, whose office it is to secrete the urine.

In consequence of the kidneys not receiving peritoneal tunics*, it is usual to regard them as extra-abdominal ; custom however appears to me to be somewhat capricious on this point, for they are certainly as much within the cavity as the pancreas, and may, anatomically viewed, with equal precision be included among the viscera of the abdomen: indeed, they cannot be

* Vide Part II. Lecture xlii. "Reflection of the Peritoneum." PART III. 
properly examined in situ but from within the abdominal cavity; where they are found a little further backward than the pancreas, immediately above (or concealed by, as the horse lies upon his back,) the small intestines. They repose, one upon either side, in beds of adipose and cellular substance, by which principally they are retained in their places, beside the bodies of the lumbar vertebræ, or rather underneath the psoæ muscles, to which they are firmly attached by this substance, as well as to the vertebræ themselves. Their anterior ends reach under the last ribs; posteriorly they come in contact with the cristx of the ilea; and to both these parts they are also tied by cellular membrane. They receive some support from the peritoneum as it passes under them, and are attached by it to the contiguous viscera; but their strongest connection is to the spine through the medium of their blood-vessels. The right kidney has peritoneal attachments to the right lobe of the liver, and to the head of the pancreas; the left has a similar connection with the blind pouch of the stomach, from the pressure of which in a state of distention it is pushed a little further backward than the right: the left is also tied to the pancreas and to the spleen.

The figure of the kidney varies remarkably in different subjects; nay, the right is commonly unlike the left, is less elongated and broader; in fact, it is a gland that does not appear to possess any very determinate form: generally speaking it may be said to represent an irregular flattened oval; but it is an imperfect oval, one side is interrupted by an inlet or deficiency of substance, technically called the notch, 
into which the blood-vessels are received. The mignitude of the gland, not les.s uncertain than its form, estimated by its weight, may be stated in round numbers to average forty ounces.

To enable me to be more particular and explicit in my description, I shall divide the kidney into its upper and under surfaces, its border, and its notch. The under surface, the part covered by peritoneum, under which is interposed a partial layer of soft yellow fat, is partially divided into two unequal lobes by a fissure running directly across from the notch towards the circumference; in many subjects the upper division is in part subdivided by a smaller fissure, which also proceeds transversely from the notch, into two portions: still it is one continuous or conglobate body, which is not the case in bears and in the cat tribe, where these lobes, being perfectly distinct, constitute it a conglomerate gland. The under surface has also a middle transverse fissure, but it is less in extent; sometimes we find two or three others, but they are small and unworthy of notice. This is the part bedded in adipose matter; but the quantity of adeps, though considerable in very fat subjects, is inferior to what is found in the herbivorous ruminant, in whom it concretes after death into a remarkably white, firm mass, well known by the name of suet. Now, that the gland is inverted, the notch is brought more into view : in some subjects this forms a considerable breach in the body of the gland, in others it makes only a part of the transverse fissure aforementioned; it is designed, like the porta of the liver, to give ingress to the bloodvessels and egress to the duct belonging to the gland. The border, thick and rounded, narrows and describes 
the segment of a circle anteriorly; broadens and extends into a larger arc posteriorly : by adverting to this circumstance, and attending to the distinguishing characters of the surfaces, the kidney of one side may be known from that of the other when they are both removed from the body.

The kidney has a capsule of its own which is every where adherent to its surfaces through the intervention of a very delicate cellular tissue, and this tissue may be traced in places into the glandular substance; at the notch processes also come from the capsule that accompany the renal vessels and nerves throughout their ramification, and serve at once as an additional tunic to them and a suitable connecting medium between them and other parts.

The interior of the kidney is most adeptly exposed to view by carrying a horizontal section through its middle in the line of its long aris. The surface exposed by this section exhibits two colors, which, though perfectly distinct, are shaded into each other by a broad intervening circle of dark red: the outer part has a brownish red tint, and is denominated the cortical substance, because it surrounds the medullary or tubular substance, as it is called, which has a carnation hue, growing lighter as it extends inward. These substances not only differ in color, they differ in consistence, in disposition, and in structure. The cortical part has an uniform appearance something like that of the liver, and possesses a texture comparatively soft and easily lacerable; the medullary is much closer and tougher in its composition, and evidently possesses a fibrous texture: they are not conjoined in any regular line, but dark-red denticular prolongations of cortical 
shoot between the lobulated portions of medullary substance.

Though the ultimate organization of the kidney cannot be demonstrated with absolute certainty, it would appear from the researches of anatomists in general that there is less speculation interwoven with the accounts commonly rendered of it than is blended with those of other complicated glandular structures. Injections sent into the emulgent arteries color the cortical substance, but are not to be detected in the medullary; a simple fact that has led to the conclusion that the former is principally composed of blood-vessels : at least this is the common result of the experiment. To pass over the detail of the means however whereby we have attained our present knowledge, the minute structure of the kidney appears to be this:-The several divisions into which the emulgent artery splits in penetrating the substance of the gland, end in a multiplied number of smaller arteries, and they form arches within the cortical substance from the convexities of which a still smaller set come off: these minute vessels proceed inward, few (if any) of them reaching into the medullary part, and end in little globular bodies that have been resembled to the acini of the liver and named the corpora globosa: a sort of arterial arrangement altogether that has been resembled to grapes as they grow upon their slender stalks. The corpora globosa were at first supposed to be cellular; but later researches afford us reason for believing that they are constituted of the vessels connected with them, which, instead of terminating in them, are prolonged and coiled into these globular forms. From the corpora globosa proceed inward in 
convergent radii, fasciculi of minute vessels named from their oftice the tubuli uriniferi; which fasciculi are so disposed in sets (commonly six or seven in number) as to admit of a resemblance to so many paps or dugs, and hence have been denominated the processus mammillares. These conical fasciculi, which take their rise in the cortical part, constitute the medullary substance of the kidney. The papilla or apices of the mammillary processes are received into little membranous sacs, varying in form and size, named the infundibula, into which the urine is distilled through the orifices of the tubuli uriniferi perforating the papillæ: the number of infundibula however is not regulated by that of the processus mammillares, for one sac may embrace two or even three papillæ. The infundibula, contracting in their course, converge towards a common centre until they open commonly by three canals into the pelvis, whose orifices are large enough to admit the small end of a common blowpipe. The pelvis is the common receptacle of the fluids transmitted through the infundibula; it is situated about the centre of the gland, almost surrounded by medullary matter, and consists of a dense, firm, membranous substance, forming an extended sac which is continuous towards the interior with the infundibula, towards the notch contracted into a small funnel-shaped outlet having one continued passage with the ureter: this continuity of parietal parts has led some to consider the infundibula, pelvis, and ureter, as one and the same extended structure; whether this be the case or not, they possess a common mucous lining that puts on the same aspect examine it in which of these parts we may.

The ureter, emerging from the pelvis, desecnds 


\section{The Kidneys.}

out of the notch and then suddenly turns backward under the posterior end of the gland, passing between it and the capsula renalis; it then takes its course directly backward, a little distance laterally removed from the bodies of the lumbar vertebræ, crossing obliquely the psoas parvus and afterwards the great iliac vessels; here it enters the pelvis by a sweep upward and outward (embracing within its concavity the vas deferens and ligamentum rotundum) and becomes included within the fold of the ligamentum latum, by which it is conducted to the lateral and superior part of the bladder; latterly, it runs in close connection from the middle of the bladder half-way to its neck and then imperceptibly vanishes between its tunics. Though we insensibly lose sight of the tube however it does not end here, for after having obliquely penetrated the muscular coat it travels onward for the space of an inch between that and the internal coat, and at length terminates by piercing the latter in the same oblique line of direction. The diameter of the ureter near its origin is equal to that of a large-sized black-lead-pencil, from which it so insensibly diminishes in calibre throughout its course that we are only assured of the fact by comparing the anterior with the posterior portion. That part of the tube not included within the broad ligament is invested with cellular and adipose tissue which binds it down in its course. It is composed of two tunics. The external one is thick, resisting, and longitudinally fibrous, and is believed to be muscular: the internal is soft and fine in its texture ; is commonly rugose lengthwise ; is loosely adherent to the other; and is constantly besmeared within with a mucous secretion to shield it from the urine. 
The emulgent arteries, right and left, arise in front of their respective glands from the sides of the posterior aorta: each enters the notch, and there divides into three, or four, or even five principal branches, which, unlike the trunk, bend in their course and then penetrate the glandular substance; the outer branches turning in contrary directions, one forward the other backward, to enter the cortical part at once; those in the middle traversing the tubular to arrive at it; in which they all subdivide and ramify as afore described.

The emulgent veins, which exceed in dimensions though not ordinarily in number the arteries, correspond in their ramification to those vessels: their principal branches, three, four, five or upwards in number, converging within the notch, there unite into one trunk which accompanies its artery and ends in the vena cava posterior.

The nerves are derived principally from the renal plexus, and very numerous they are: notwithstanding their numbers however, and notwithstanding the acute pain which accompanies active inflammation of it, the organ in the healthy condition is by no means remarkable for its sensibility.

\section{The Capsule Renales}

Are two small, elongated, irregularly-formed, brownish bodies, a right and a left, placed opposite to the kidneys, between those glands and the spine. The right, the longer one, lies in contact with the posterior vena cava, and reaches forward to the liver; the left, the broader one, and rather obliquely placed, is opposed to the aorta, and anteriorly is contiguous to 
the pancreas: they are respectively connected to these several parts by loose enveloping cellular substance, and are furthermore retained in their places by the peritoneum which covers their under surfaces. Their magnitude varies with age: in early fotality they are equal in volume to the kidneys themselves; their subsequent grow th however being less rapid than that of the kidneys, this equality in the course of time becomes destroyed. Their outward borders are partially cleft by several little notches which give them a lobulated appearance. They possess tunics of their own of condensed cellular membrane which penetrate beyond the surface and enter into the composition of their interior.

When lengthwise divided by a perpendicular section, the renal capsule shows a palish brown substance above and below, which is interspersed with vessels and nerves, is soft and uniform in its texture, and composes full two-thirds of its whole; and in the middle a longitudinal whitish part, which, though of a very loose texture, is evidently not a vacuity; for, after some yellowish fluid it commonly contains is expressed there still remains a loose or cellular structure. The fluid expressible from this part is said, to vary in its appearance and quantity with the time of life : in the foetus it is said to be red and abundant, yellowish and sparing in the adult, yellower still and more scanty as age advances. This body receives two or three small vessels from the emulgent artery or aorta or from both; and these have their full proportion of veins. They have also an adequate supply of nerves coming principally from the renal plexus. What the disposition or arrangement of these several component parts may be remains yet to be developed; M. GIRARD says, con- 
cerning them, that, although these bodies are plentifully furnished with blood-vessels and nerves, "ils n'offrent dans leur organisation aucune disposition qui puisse faire presumer une sécrétion particulière."

The physiology of the capsulæ renales remains to this day an unsolved problem. Not one fact has been related to light us in the investigation. All that HALLER's indefatigable research and penetrating mind could discover for certain about them, was, that they secrete a fluid more required in fotal than adult life, and that their functions are probably important since they are found in so many animals.

\section{The Bladder.}

THE musculo-membranous bag that receives the urine from the terminating orifices of the ureters.

The bladder occupies the middle and inferior part of the pelvis, takes the oblique axis of that cavity, and rests upon the symphysis pubis, having the rectum over it in the male, the vagina in the female. In the undistended state this viscus is wholly confined to the pelvic cavity; but when full its fundus advances before the pubes into the abdomen, the advancement being in ratio with the distention: in the foetus it rises still more into the abdominal cavity, as a necessary consequence of its proportionally greater development, and the narrowness and shallowness of the pelvis at that period.

In a state of distention the bladder is pretty regularly pyriforin, and its parietes are thin and semi-transparent; but when completely empty it assumes the spheroid figure, and becomes thick and has no vacuity intermally: in fact, it is in that state a little white, 
round, frm, and, I may add, solid body. Its capacity will vary much in different subjects; and this we have reason to ascribe principally, from a correlative fact in human anatomy, to the habit the animal had of retaining the urine; for it is found that women, whom we know from motives of delicacy are oftentimes compelled to restrain their inclinations to void their urine, have generally larger bladders than men: and now and then in such subjects bladders are found of extraordinary volume.

The bladder is connected by loose cellular adhesions to the pubes, the walls of the pelvis, and the rectum in the male, the vagina in the female; but it is balanced in its proper place and position by two broad productions of peritoneum expanded across the cavity from its sides to the walls of the pelvis, called its ligamenta lata. These are formed thus: the peritoneum being reflected upward from the recti muscles upon the body of the bladder about its middle, proceeds forward upon the fundus, then turns back again and covers still more extensively the upper surface; at the sides these (under and upper) layers of the membrane, meeting together, unite and afterwards proceed in union to the walls of the pelvis, where they once more split and take opposite directions. The middle portion of the upper layer of peritoneum is reflected upward from the hind part of the body of the bladder upon the rectum in the male, the vagina in the female, and thus a peritoneal pouch is formed between the two. In addition to these attachments, the neck of the bladder is connected to the pubes by a thin fibrous expansion, the ligamentum triungulure; and the fundus receives the insertion of two 
round cords which run within the folds of the broad ligaments, named the ligamenta rotunda.

The bladder may be distinguished in description into its fundus, body, and cervix or neck. The fundus is the round and prominent part presented forward, is completely covered by peritoneum, occasionally protrudes into the abdomen, receives the attachments of the round ligaments, and the insertion of the degenerated urachus; (which is in the fotus a urinary conduit continued along the umbilical cord to the membranes of the womb). The body is the bulky or capacious part of the bag; only one-third of its under surface is covered by peritoneum, nearly the whole of its upper; it is supported by the broad ligaments, has along its sides in the male the vesiculæ seminales and vasa deferentia; and behind grows suddenly contracted and ends in the neck. The cervix is the small, circular, posterior part of the bladder; it has a light-pinkish hue, and is comparatively thick and substantial to the feel; it has the triangular ligament fixed to it, and is united at the opposite end to the urethra.

The bladder has three coats : an external one which is derived from the peritoneum; a mucous membrane to which it owes its integrity and principal consistence ; and a diffuse fibrous texture interposed between the two which is of a muscular nature. The peritoneal coat, which forms but a partial covering, is smooth, polished, and moistened with serous exudation externally; but rough and pilous internally, when it is torn from its adhesions with the muscular: in a word, it in nowise differs in its properties from the peritoneal tunics of the abdominal viscera. It serves to hold the bladder in its proper place and position, and to a 
certain stretch to counteract preternatural distention of it.

The muscular coat, though it may be demonstrated through the pellucid peritoneum, is brought more distinctly into view by stripping off that membrane, to which it is closely and firmly attached by fine cellular tissue. Pale fasciculi are then seen running irregularly in a longitudinal manner; and, underneath them, others which are smaller, taking an equally irregular course in the circular direction : in the empty or halfdistended state of the orman both these orders of fibres assume spiral courses; this enables them to bear considerable extension without the risk of rupture, while their cellular comnections, being loose, admit of their being drawn apart with equal facility. The longitudinal fasciculi are thickest about the fundus, where they all converge to its central point; the circular are strongest around the cervix : the former exert a special contraction in forcing the urine towards the neck, constituting what has been called the detrusor urina; the latter, which are mingled with cellular tissue and some small veins, giving them the feel of greater substance than they really possess, form the sphincter vesica of those who (with no more reason in my opinion) make a separate muscle of this part. By the united and regular contraction of both orders of fibres the parietes of the bladder may be drawn towards one common centre so as pretty equally to diminish its capacity in every part, until the cavity is altogether annihilated.

The mucous coat shows itself exteriorly in the interspaces between the fasciculi of the muscular, covered only by the interposed cellular tissue which serves as the common uniting medium of all these tunics. When 
the bladder is empty the contractions of the muscular throw this coat into ruga, which in cases of extreme contraction assume rather a remarkable appearance: the cellular tissue interposed between this and the middle tunic is loose to admit of this, and, from its being pressed into a pretty regular layer wherein the vessels and nerves of the organ ramify, it is described by some as the nervous coat. The internal membrane is white, soft in its texture, and highly organized; besides which it possesses numerous follicles from whose excretory pores issue a plentiful mucous secretion to defend it from the acrimony of the saline inatters contained in the urine: this mucous matter being perpetually washed off the surface by the urine is kept continually renewed; and it is occasionally voided in considerable quantities, especially when any calculi or other irritants are present in the bladder. About an inch before the cervix, in the sides of the bag, are seen the mouths of the ureters, whose oblique insinuations in perforating the parietes operate in preventing any reflux of the urine at the time that the bladder is contracting, and thereby have completely the effect of regular valves. 


\section{LEC'IURE LIV.}

\section{On the Physiology of the Urinary Organs.}

THE kidneys are entitled to our earliest consideration as being the organs in which the fluid we call uirine is elaborated from the circulating blood. Were it required of us to adduce argument or evidence in support of so palpable a truth as this has become in physiology we need not seek long for either. The bare anatomical examination of the urinary apparatus altogether, conducted by one conversant with the general principles of the animal economy, is perhaps enough to raise suspicions of such a nature in the mind; but when the emulgent artery comes to be compared with such vessels as the hepatic, the coronary, and the bronchial, which are considered to be the nutrient arteries of the parts to which they are respectively going, and these parts come to be individually contrasted in bulk with the kidncy, it does not appear unreasonable to draw an indirect inference at once that secretion of some kind or other must be carried on in this body, since the blood sent to it far exceeds the quantity necessary from analogy for the purposes of nutriment and repair. Setting aside all hypothesis 


\section{On the Physiology of the Urimury Organs.}

and analogical deduction however, we have indisputable evidence of the facts, that urine is produced in the kidney, and that it passes thence through the ureter into the bladder. Diseases that suppress the functions of the kidney, caculous concretions obstructing the passages, and a fortiori ligatures upon the ureters, arrest the urinary discharges: during any such morbid or unnatural condition of these parts not a single drop of urine is found in the bladder; in the former case there is none secreted; in the two latter the fluid collects between the place of obstruction or ligature and the kidney, distending and dilating even to disruption the mammillary processes, infundibula, pelvis, and ureters.

In this manner may be demonstrated the secretion of the urine in the kidneys and its passage into the bladder; a knowledge no sooner attained by an inquiring mind than the questions, how and by what particular parts of the organ the secretion of it is effected, naturally follow in this interesting investigation. So long however as our knowledge of the minute anatomy of secreting organs remains so imperfect, so long as we continue unable to connect particular glandular structure and arrangement and action with the particular nature and composition of the secretion resulting therefrom, so long must these questions go unanswered; and though in the instance of the kidney, as I stated before, we have been more successful we think than in most similar researches, still the amount of this information does not appear to have been in the least enhanced by the circumstance. We suppose that the urine is secreted within the corpora globosa by the minute ramifications of the emulgent arteries, and that the tubuli uriniferi receive it from these bodies; and we 
know that afterwards the infundibula collect it from the mammillary processes and discharge it into the pelvis, whence it is carried by the ureters into the bladder.

The blood is here, as everywhere else, the pabulum of secretion; but it would appear from some facts touching the process and from the known composition of the urine that the serum was the part mostly expended in its production. Water forms the bulk of both these ftuids; and they contain saline substances of a similar nature. What are called the serous exhalations in the body, and especially that from the membrane lining the bronchia, influence to a certain extent the urinary secretion; hence it is that dropsical subjects, in whose systems there is such a disposition to serous effusion, make but scanty discharges of urine; and hence it is that by setting up a reversion of action in farour of the kidneys we get rid of the dropsy. But the most remarkable sympathy of function of this description exists between the skin and the kidneys, and it is onerthat also tends to show that urine is principally obtained from the thin or aqueous parts of the blood. In warm weather when animals perspire much there is very little urine voided, and the reverse of this happens in cold weather; men have an opportunity of remarking this in their own persons, and it is well known to coachmen and others in the habit of riding or driving that horses who sweat profusely seldom stop to stale on the road; indeed so sparing is the secretion in some instances that when such horses are put into stables to induce staling they void so small a quantity that they are occasionally imagined to labor under what is called "a stoppage." This then is what we understand by sympathy between 
the skin and the kidneys; in this manner is there a sort of equilibrium of function maintained between the emulgent and perspiratory vessels. Again, it is found, cateris paribus, that the quantity of urine will be regulated by the quantity of water drunk. The horse who drank within the space of a few hours thirty-eight gallons of water*, shortly after deluged his stall with urine; for, as I observed before, this prodigious ingurgitation was not followed by purging, though had he been taken out and sweated a considerable proportion of it would have passed off by perspiration; and this is a case that not only throws light on the aqueous nature of the secretion, but it also shows us what an enormous bulk of fluid may be excreted from the system in a very short space of time in the form of urine. We might a priori suppose that the serum would also vary its proportions with the quantity of fluid imbibed: Professor Coleman many years ago made this experiment to determine the point. He kept a horse for several days without any fuid whatever; and afterwards permitted the animal to glut himself with drink. Now the horse was bled during the continuance of thirst, and at different intervals after he had drunk five or six pails-full of water; and yet the Professor could discover no variation whatever in the proportion of the serum to the crassamentum. The urinary discharge was but little during the time of drought, but returned copiously on a sudden after the animal had satiated his extreme thirst: from which it appears that the watery constituents of the chyle are not permitted to continue in the circulating mass, but are 
speedily separated therefrom; and that the kidneys in cold weather, but the skin when it is warm, are the principal emunctory for its discharge.

The shortness of the intervals between the taking of a copious draught and the consequent augmentation of this secretion, and between the swallowing of food and the detection of that food by certain properties it happens to possess in the urine, has led some physiologists to think that there must exist a more direct passage to the bladder than the regular round of lacteals, circulation, and kidneys. No such special passage however has ever been discovered; and even if we iniagined that there were absorbents that could serve such an office the hypothesis must be supported by the very serious and responsible admission that there exists a power in the alimentary canal to form urine. Independently of all this however, the bare fact that obstructed ureters and an empty badder are consequent and unalterable conditions of those parts, though the evidence be but negative, is sufficient to refute the above conjecture.

This secretion is one that is constantly going on; by night as well as by day, during hot weather as well as cold, whether little or much or even no water at all be drunk, though its quantity will be considerably influenced by these circumstances, urine is continually passing from the kidneys into the bladder : of this we may at any time convince ourselves by leaving a catheter in the bladder, or by referring to those cases on record, both of malformation and disease, in which the ureters have opened upon the surface of the human body. It is not so with secretions in general; we have 
reason to believe that supplies of bile, semen, pancreatic juice, \&c. are only furnished as occasion requires.

Fear and anxiety of mind have a remarkable effect on this secretion in the human subject, and the same influence is equally striking among animals. It is by no means an uncommon occurrence upon a race-ground to meet with a racer and his jockey who are said to be "leaky before starting;" on which occasion I should argue that the feelings of the horse and man were not widely different. Again, what fox-hunter has not seen many horses in the field staling at the time that the hounds were finding in the thick of the covert? or who has not seen a cur do the same thing at the sight of a horsewhip?

Another distinctive character of this secretion is the extreme variableness of the product. In health this is too remarkable to have escaped the notice of the most casual observer; and in disease it may be ranked among those phenomena which remind us how little we yet know of primary or proximate causes. It may be observed in a general way that the greater the quantity of urine secreted, the paler, less acrid and odorous it is; and so far imbibition affects its quality as well as quantity: indeed the urine that rapidly follows a copious draught is in so diluted a state that it has the appearance of being little else but pure water, and so has been denominated crude urine or urina potus. But the urine which is voided a few hours after feeding, when the food has had its chyle extracted by the lacteals, or that which has been long secreting, is high-colored, acrid, and emits a pungent fetor; it is in fact strongly impregnated with the urinous 
properties and such as a chemist would choose for analysis were he about to examine its composition: this has been distinguished as the urina chyli or concocled urine. But, independently of this variation, which may be compared to simple dilution of it, the quality of the urine will be affected by the kind of food consumed by the animal, and in some instances in so remarkable a degree partake of its properties that we may determine the nature of the ingesta simply by examination of the urine. In the human body, garlick, onions, asparagus, carrots, and some other vegetable substances, impart to this fluid peculiar recognisable fetors; and beet root and madder alter its color: I do not know that any one has made any such observations among horses, but $\boldsymbol{I}$ should not hesitate to predict similar results were their food materially altered; horses in general are fond of carrots, and will feed upon Swedish turnips, beet root, and mangel wurzel, so that the experiment might easily be made. The effects of change of diet however are in no instance more strikingly illustrated than in diabetic persons when the plan of treatment consists in restricting them to animal food: Professor Coleman once kept a horse for many days upon flesh balls, but I am not aware that any particular notice was taken of the urine, since the experiment was made with other views than what engages us here. Certain medicinal substances also are known to affect the urine: aloes heightens its color, turpentine imparts to it a terebinthine odor, but what impregnates it with the most pungent and offensive fetor of any thing that I have ever given is the balsam of copaiba; during its exhibition the stable is rendered 
so obnoxious that any one who is acquainted with the balsam instantly recognises its peculiar unpleasant odor corrupted by the urinary and alvine excretions.

But we have yet to take notice of the greatest modifications to which this secretion is subject, those that are symptomatic of or consequent upon certain diseases. Some of these variations were particularly made the subject of observation by the ancients, and have in former days furnished empirics with tests of a very imposing nature of their pretended superior skill in curing many internal disorders; the same have also served to weave a cloak of ignorance and deception for our farriers in which they seldom fail to wind up their accounts to the satisfaction of their confiding employers: on the other hand, modern veterinarians in rejecting the popular notions of their predecessors of seeking for the cause or nature of the disease in the appearance of the urine, seem so completely to have laid aside the practice that they leave themselves unacquainted with what few morbid alterations the urine really does undergo. We know in the human subject that what is called the lateritious sediment denotes the decline of acute disease; that the urine is colorless in hysterical affections; that it is deficient in its earthy ingredient pending gout, but abounds in that substance where there exists mollities ossium: but I know of no such connected observations in veterinary pathology. In diabetes the urine of horses, as well as that of men, may be boiled down to a saccharine matter resembling molasses or treacle. Occasionally we meet with bloody urine : this may proceed either from excess of vascular action, or from injury to the glandular substance or 
any of the mucous surfaces over which the urme has to pass; and in cases where these passanes throw off more than ordinary secretion or run into suppuration, mucus or pus will be found mixed with the urinary discharges.

Having submitted these leading facts in regard to the process itself, and the variations that have been noticed in the quantity and quality of the fluid secreted, we now come to the consideration of the grand question, for what specific design in the animal economy such a secretion was instituted. Considering the aqueous constituent of the blood as the material by which the temperature of the system is kept down by evaporation from the surface of the body, the kidneys have been vicwed as parts performing a subordinate function to the skin, simply as a secondary or minor part of the cooling apparatus. The theory, and a very rational one it is, is this. The animal requires a certain quantity of perspirable fluid in the blood for the purpose of conducting heat from the surface during exercise, hot weather, or any other influence that may raise to cxcess the temperature of the body; but as this fluid is sensibly perspired only on such occasions, there required an emunctory to get rid of it when it is not wanted for this purpose, which emunctory is the kidneys. Moreover, it is urged in favour of this very plausible theory that this explains why the urinary secretion is not required in the foetus; namely, because the surface of its body is surrounded by fluid, and a fluid invariably of the same temperature. For my part, I look upon this as I do upon that opinion which ascribes the office of the spleen to be to prepare blood for the liver: I do not see in any other instances that such complicated and expensive means are pro- 
vided solely to bring about such comparatively simple ends, and I feel more disposed to dissent in the present case because another office, and in my opinion one infinitely more consistent with the indisputable importance of the organs, has been assigned to them. Is it in accordance with the other operations of the animal economy to set down such a complete organic structure with all its appendages as nothing but a waste-but to the skin, a part with which in structure it will admit of no analogy whatever, and between whose secretions there is just as little affinity? Is it likely that two organs should have been so intricately constructed and organized and placed where they are purely for the purpose of acting in subserviency to a part of such comparative unimportance and simplicity of structure as the common covering of the body? I should unhesitatingly answer, No! and rather say, with others, though their assumption may be one that does not directly admit of proof, that the principal use of the kidneys is to separate certain matters from the blood, under the form of urine, which have become excrementitious either from having served their ends and become worn out, or from their retention in the system being actually prejudicial. We know that there is a continual change of material going on throughout the body, that every part becomes renewed within a certain space of time, and that certain parts that grow useless become absorbed and carried into the system along with the old materials : conscquently there required, not merely a discharging channel, but an organ having the power to select these materials out of the circulating mass and elaborate them into a form convenient for expulsion. But does the urine afford any evidence of such a material? Yes ! it yields a substance named urea, which excecds in quan- 
tity all the other substances contained in it, to which its color, odor, and every other characteristic property it possesses is found to be attributable; and this urea we imagine to be the said excrementitious material so changed that it may be dissolved in water and without difficulty discharged through the urinary passages : at the same time this water in the blood, which serves to hold the urea in solution in the form of urine, is as occasion may require at the disposal of the skin. So far then I am ready to admit that the kidneys are connected in function with the skin; only let it be remembered that this is secondury to the important part they perform in their rank of complicated glandular structures.

Healthy urine, as every one knows, is a fluid of an amber color, of a sharp saline flavor, and of a peculiar, pungent, offensive odor; but its color, flavor, and odor will vary with the circumstances already mentioned which influence its secretion. Besides urea, of which the lateritious sediment principally consists, it contains albumen, commonly some mucus, and different salts ; all which, in extremely variable proportions, are held in solution in an equally variable quantity of water. According to M. GrRard, the salts found to predominate in the urine of the horse and ox are, carbonate of lime and soda, muriate of potash and soda, and benzoate of soda.

THE BLADDER, though not absolutely a necessary, is a very useful part of the urinary apparatus. The secretion of urine being incessant and its transmission through the ureters uninterrupted, if there had been no bladder the urine must have been continually dribbing from the animal, thereby keeping up a constant 
fetor, irritation, and excoriation, besides other annoyances, all which may be better conceived than described: malformations of this description are on the records of human medicine; but I have never met with any such accounts relating to the brute creation. The urine then as fast as it collects in the pelves of the kidneys from the terminations of the infundibula, is received by the funnel-shaped mouths of the ureters, conducted by those tubes into the bladder, and therein deposited by each of them drop by drop. As it continues to receive this guttatim influx of urine, the bladder insensibly increases in volume; its sides separate and dilate, the rugæ vanish from its interior, and the parietes of the bag grow thinner and thinner until they become stretched and attenuated even to a degree of transparency: this dilatation in the longitudinal diameter is equal to double what it is from side to side notwithstanding the former only takes place in one direction; for the cervix of the bladder being a fixed point will not admit of elongation, whereas the fundus being only loosely attached keeps gradually rearing itself forward, passes the pubes, and protrudes into the cavity of the abdomen. Urine that has once entered the bladder cannot possibly return: the oblique insinuation of the ureter between the fibrous and inner tunics forms so effectual a valve that even air cannot escape it, and what adds to the beauty and effect of this valvular contrivance, is, that the obstacle is strengthened in the same ratio in which the resistence caused by distention is increased. Whenever a certain quantity of urine is collected in the bladder a sense of uneasiness is created which the animal well knows is the warning to stale: if he does not obey this 
inward call, and particularly if his attention be diverted at the time by some unexpected incident, the feeling will die away and the interval of retention be protracted; but this remission of the act, although it admits of considerable latitude to suit the convenience of the animal, cannot be prolonged beyond a certain limit without inducing an irresistible impulse to yield to the efforts of nature. This desire to empty the bladder will not be regulated solely by the degree to which the organ may be distended; it will be considerably influenced by the habits the animal may have acquired of staling at longer or shorter intervals. It is therefore by a voluntary power that we expel urine from the bladder; in a state of health no involuntary discharge ever takes place; and the act is performed in this manner:-

The animal (supposing him to be in action) first stops and places himself in a certain convenient position; he then takes in a deep inspiration, by which the diaphragm thrusts the abdominal viscera towards the pelvis and keeps them pressing upon the bladder; lastly, still holding his breath, by the exertion of the abdominal muscles (particularly the transversales) this pressure is greatly increased, and by the joint-contraction of the muscular coat of the organ itself is rendered available in its evacuation. It would appear from what we have learnt by experiment and from pathology, that these powers acting singly are insufficient to the end: if the bladder be paralysed all voluntary efforts to discharge the urine are unavailing, and so are the contractions of the organ itself when the abdominal parietes are cut away. During the continuance of the act the impetus of the stream may be increased 


\section{On the Physiology of the Urinary Organs.}

at pleasure, or the current may be projected by jerks as it commonly is towards the conclusion : the former is the effect simply of increased abdominal effort, the latter is ascribable to the superadded action of the accelerator urinæ. But a few drops make their appearance or the stream is but small at first in consequence of the time taken up by the voluntary powers in overcoming the opposition at the cervix of the bladder; but, when once this part is dilated the urine flows in full stream and continues so to do without further effort, as is seen by the relaxation of the flanks and the recommencement of respiration, the operation being continued by the bladder alone. Towards the end of the act the stream becomes again diminished, and the last portions of urine are so feebly expelled from the bladder that their discharge from the urethra calls forth the contractions of the accelerator urinæ. Through this same muscle also the animal has the power of suddenly arresting or interrupting the stream during micturition, and of resisting an urgent impulse to stale; for it will so effectually compress the urethra all the way from the bulb to its termination that no effort the bladder can make can have any effect.

Whatever may be the amount of the contractile powers of the bladder they are to be considered as altogether without the pale of voluntary influence; there is an inherent or natural tendency in the organ to contract, as is plainly proved by leaving a catheter in it, and in cases in which there exists paralysis of its neck. We can understand how the urine is discharged from this organ by certain auxiliary forces, and how it is retained or the stream at pleasure arrested by similar means; but it does not appear so casy to demonstrate 
the contrivance whereby the urine is kept from flowing spontaneously, without admitting (what we have not been able to develop to our satisfiaction by the knifi) the existence of a sphincter vesice: if the part really does possess this faculty and is the sole and efficient cause of the retention, I would rather refer its power to the smallness of the neck and to its being made a fixed point, circumstances which must materially favor its action, than to any superaddition of muscle which does not admit of satisfactory demonstration. That it is no voluntary effort is proved by its unceasing constriction during sleep; that it is not attributable to clasticity is proved by its relaxation after death; that it is a muscular act is vastly more feasible in a physiological than an anatomical point of view : be it what it may however we must acknowledge its contractility or we cannot account for the closure of the bladder.

Finding that the urine voided at different intervals is dissimilar in its properties, physiologists in general believe that it undergoes some alterations during its stay in the bladder; I do not apprehend however that this is a change of any specific nature, but purely one referable to the circumstances under which it is therein lodged. The urina potus indeed appears to pass through the cavity the same in composition as it was when received from the kidneys; but that which is slowly secreted and is long retained in the bladder, becomes turbid, acquires a peculiar smell, and is found to be charged with animal matter: properties (as far as relates to the bladder) ascribable to some absorption probably of its aqueous parts, and the admixture of the secretions from its internal surface. 


\section{LECTURE LV.}

\section{SHALL commence this lecture with a few observa- tions on}

Diuresis and Diuretic Medicine.

$\mathrm{By}$ diuresis is meant discharges of urine recognised to be inordinate in quantity and in the frequency of their return: diuretics are the substances which promote these discharges. The secretion of urine may be augmented in two ways; either by producing an actual augmentation of that (the aqueous) constituent of the blood from which the bulk of urine is obtained, or by introducing a substance into the system which will turn out to prove a direct stimulus to tire kidneys themselves. I observed in the former lecture that liquids passed out of the system in three ways, by cutaneous perspiration, by pulmonary exhalation, and by urine; and that augmented drinking was commonly followed, as a natural consequence, by augmented urinary discharges, a fact that has given rise to a common practical observation, "that water is the best diuretic;" this however, it will be seen, can only hold true in a conditional sense, for if the cutaneous or even the pulmonary surface happen to have the 
determination of blood in its favor, the kidneys may make no more than the ordinary quantity of secretion though the imbibition have been considerably more than usual. This is but rarely the case however in horses. Man, we know, in warm weather throws off the largest proportion of fluid by the skin and thus wards off the heat from his naked surface : animals having hair dispose of their fluids principally through the other channels; the dog cools himself by pulmonary exhalation; the horse, who rarely perspires but under exertion or pain, has probably of all other animals the most susceptible kidneys.

Substances that promote the secretion of urine may be either of an alimentary or a medicinal nature. Mow-burnt hay, foxy oats, and all sorts of high-dried and fermented provender prove more or less diuretic to horses unhabituated to such food; and in a general way this forms one objection to their use, for nothing tends to debilitate a horse more than frequent and long-continued excitation of the kidneys. Diuretic medicines are both great in nymber and various in kind, but they require on our part the exercise of more precaution in the exhibition of them generally than judgment in the individual selection of them: this is not the case in the human subject, but it is a practical point to be minded by the veterinary surgeon if he would eschew those evils into which the farrier, the horseman and his groom, are every day the unknowing authors of. The neutral salts, particularly nitre; the alkalies, uncombined or as they exist in soap; the balsams, especially that of copaiba; the terebinthates in a remarkable degree; lytta in a violent degree; savin, oxy-muriate of mercury, nitrous œther, and 
some others, all possess diuretic properties; in ordinary practice however we seldom use any others but soft or hard soap, yellow resin, and common turpentine*. The prompt action of diuretics and the debility of system they have a tendency to induce (though that is an effect that can only be insured at the expense of violent derangement if not progressive disorganization of the secretory structure of the kidneys) have led to their use in cases of plethora, and in febrile and pulmonary affections; but they are chiefly employed in dropsies of all kinds. If a horse is brought to us purely for having anasarcous legs we prescribe for him a course of what we call diuretic medicine, and we know for certain that his legs in the course of a few days will become fine. Now, what is the theory of this? Is it to be explained by saying that the kidneys are stimulated by the medicine to secrete more than the ordinary quantity of urine, and that the absorbents, as a natural consequence, set to work to make up for this inordinate abstraction of the watery parts of the blood by taking up that deposited in the legs; or is it that this medicine, which we call diuretic, stimulates the absorbents, and that the increased action of the kidneys is ascribable to the augmented influx of lymphatic fluid into the circulating medium? A know-

- Re Saponis duri vel mollis 3ij.

Resin. Flav. Pulv. $3 ß$.

Ol. carui 3i. m.

Ft. Bol. omni nocte exhibendus.

R. Terebinth. Vulg. $31 \xi$.

Ol. Juniperi $3 i$.

Pulv. Glycyrrhizæ q. s.

M. ft. Bol. alternis diebus dandus. 
ledge of the bare fact will serve us for every purpose of practice; and therefore I shall not take up this lecture by launching into any theoretical solution of it; but proceed forthwith to the

\section{Diseases of the Uninary Organs.}

THE general abuse of diuretic medicine, coupled with the known susceptibility of the organ itself, renders the kidney the seat of frequent disordered function, and every now and then of violent and destructive disease. The disordered function is probably the result of increased action, which may or may not amount to inflammation; if it do not in the first instance the frequent repetition of the stimulus does not fail to give rise to it, most likely in the chronic form, and this languid inflammation, which within certain limits would still die away on a total discontinuance of the exciting cause, aggravated from time to time by the same injurious diuretic influence, becomes at length established, and then never leaves the part until it has even defeated its own progress by inducing disorganization, induration, schirrus, ulceration, and suppuration, \&c.: this is a subject however on which we are in the existing state of veterinary medicine wofully uninformed, and therefore we must content ourselves for the present with a knowledge of the more tangible form of renal disease.

\section{Nephritis.}

Acute inflammation of the kidney or kidneys I believe to be uncommon, viewed as a spontaneous affection; by which I mean, a disease arising without any apparent or assignable cause: its origin may gencrally be re- 
ferred either to excitement of the organ, or to some sprain or other injury about the loins; a prudent practitioner therefore will not fail in cases of this nature to inform himself of the circumstances under which the disease has made its appearance, and he will find that this knowledge will contribute vastly to lighten him on the road of subsequent investigation. The ordinary symptoms are--The horse stands wide and stiffly with his hind legs. In walking he shows an awkward straddling gait behind : this is more remarkable when he turns round or is put into a trot, actions he manifests unwillingness to perform. He probably expresses tenderness in the neighbourhood of the kidneys by shrinking from pressure upon the loins. The urinary discharges are suppressed altogether; or, should any make their appearance, they are frequent, sparing, high-colored or bloody, or perhaps mingled with pus: all which will depend on the stage and violence of the inflammation. The bladder is empty. The system is suffering from more or less febrile disorder. The pulse is small and very quick.

The common causes are : the frequent use of acrid diuretics, feeding with certain kinds of diuretic provender, or even the long continuance of certain mineral waters; overweighting, leaping, suddenly turning or pulling up upon the haunches during the gallop or fast trot, by which the loins are sprained or otherwise injured; lastly, the irritation excited by calculous matters within the pelvis.

The diagnosis, being a point of great importance in the treatment, ought to be carefully made out before we take any decisive therapeutic measures. We are most likely to confound nephritis with cystitis: here 
the particulars of the history of the calse may do mach for us: then we must advert to the peculiarity of posture and gait, and the totul suppression of urine or state ahnost equivalent thereto. Should there be constriction at the neck of the bladder, in which case also no urine will be discharged, that viscus will be found on examination prodigiously distended. I know of no sign that will lead us with any decree of certairty to infer whether one or both kidneys be affected; or, if but one, which of them : and it is of no great import further than, in the case of but one bcing affected, the circumstance will tend to puzzle us in our diagnosis.

I believe the most common termination of cystitis to be suppuration, not of the cortical part but of the mucous passages, the infundibula, pelvis, and perhaps the ureter; though it may end in abscess of the entire gland. Condensation, induration, and schirrosity of the glandular structure I havo seen specimens of in the state of preparation. I have heard of gangrenous and mortified kidneys, but I never saw one.

The indications in the treatment are, to subduc the feverish excitement in the system, and to combat as closely as we can with the inflammation in the gland itself by remedies particularly directed thereunto, and by withdrawing every thing that might have a tendency to aggravate or continue it. Free use ought to be early made of the lancet; from four to eight quarts of blood may be drawn. And that being done, we are to lose no time in administering a full dose of purgative medicine: during the interim that thes remains inactive we may (after the scylola have been removed from the rectum) inject at short intervals 
copious clysters, warmer than we are in the habit of using, which will have some beneficial effect as an internal fomentation and will probably accelerate the action of the purge. Hellebore will also be found serviceable in this interim, repeated every four or six hours until we have induced nausea, a very desirable state in this disease. The pulse having been weakened by the use of the lancet and diminished by the exhibition of hellebore, we may safely commence counterirritation. The common notion that blisters "do more harm than good" appears to be founded in error; for it has been (I may say I believe) proved that vesication precedes absorption; if, therefore, a blister be washed off as soon as it begins to rise we derive all the benefit without any of the ill effect that the absorption of lytta (which is an acrid diuretic) would be productive of. But, if this be questioned, the mustard embrocation may be spread upon the loins instead of the blister. The more diluent fluid taken (barley water, oatmeal gruel, linseed tea, or even plain water) the better; but I would not drench the horse with any : at the same time we should take care to get rid of as much of this fluid by the skin as possible by warm clothing; though it pass off by urine however (which most of it probably will when the inflammation is on the decline) it will still prove beneficial by diluting the salts and thereby diminishing the acrimony of the secretion. Scrupulous abstinence should be enjoined from the use of any food or water having diuretic properties.

\section{Diabetes.}

THIS is commonly classed among renal disorders, inasmuch as the urine is found to possess a particular 
sweet taste or saccharine quality, and to be voided not only in unnatural profusion but at more frequent intervals than in health. Though diabetes is an infrequent malady among horses we cannot make a plea of this circumstance for passing it over in silence: no one can tell how soon he may be called to treat a case of disease of rare occurrence, and if then he feel himself unprepared, though he may administer placebos to his employer, it is rather vexatious to have afterwards to reflect that his unfortunate patient, brute as he is, might have been saved had he fallen into other hands. Though the saccharine property of the urine and profuse staling are the circumstances upon which we must rely to lead us to the detection of diabetes, it appears that neither of these symptoms singly is essential to its existence; for, in the human subject the secretion has been known to be saccharine and yet remain unincreased, and to be augmented but inodorous and destitute of saccharine matter, a form of the disorder that has been distinguished from diabetes mellitus by the epithet insipidus: I am not certain however whether we are warranted or not in making a like distinction, and so I shall collect what information I can about the first of these disorders.

The urine is discharged so often and in such immoderate quantities that it has been known in the human subject to exceed in weight the ingesta (solid and fluid) altogether; and Dr. RoLlo, who has given an account of such cases *, detected a daily loss in the weight of these patients : indeed, some such notion seems to have been entertained by those who named the complaint diabeles. 
Thirty-six pints of urine it is said have been voided in one day by a diabetic man; I am not in possession of any similar particular's in regard to horses. The urine itself is limpid, light-amber-colored, peculiarly odorous, sweet in taste, and yields, by a process of evaporation, a saccharine matter possessing properties very similar to treacle or molasses. These alterations in the quantity and quality of the urine are accompanied with other symptoms, which, should those signs escape our notice, (or that of the groom rather) are very likely to call our attention to the presence of the malady: these are, remarkable voracity of appetite, intense thirst, tardy febrile action, dryness and roughness of coat, falling-off in condition, and dullness and unwillingness to move, followed by general emaciation, extreme debility, and even death. Its attack is imperceptible, its progress slow and commonly for some weeks continues unobserved, for the animal may have the malady for a considerable time without shewing any very evident signs of its existence. I do not know that any analysis has been made of the urine of diabetic horses; but Drs. BlACK and Rollo have found that zirs. of saccharine matter was afforded by a pint of that voided by a diabetic man; whereas, a pint of ass' milk yielded but 33. and the same measure of cow's milk only half that quantity.

To enter into the theory of this disorder in detail would take up too much of the present lecture; I shall therefore confine myself to one or two current observations. These urinary phenomena by some physicians are regarded as the result of morbid action of the kidneys, or of an inflammation in them of an indolent or specific character, ascribing to the organs a power of converting 
certain parts of the blood into saccharine secretion, the same as the mamma appear to do ; the answer commonly given to which hypothesis is, that if the kidneys can assume the operation of one gland, why not of another, and secrete bile and semen, \&c. The opinion more generally entertained at the present day is, that this saccharine secretion has its origin in some defect in the digestive or assimilatory process ; and this opinion is supported by the facts-that sugar is one of the principal and earliest products of digestion and that chyle is known to contain a considerable proportion of it; that the vegetable nature of diabetic urine is proved by its turning acidulous instead of running into putrescency as common urine does, and by its being fermentable with and sometimes without yeast; that a peculiar stomach-affection usually precedes and accompanies diabetes; that an animal diet or abstinence from all vecretable and sugar-yielding substances will speedily remove the characteristic symptoms; and that dissection has not developed any morbid alteration in the kidney but what may be referred to simply increased glandular action, viz. some enlargement of the gland, especially of the uriniferous portion of it.

The exciting causes of diabetes it appears we know but little about. If it can be proved to originate in deranged function of the stomach or other of the chylopoietic organs, we must look for the cause either in the nature, quality, \&c. of the provender, or in some kind or other of organic dyspeptic affection. On the other hand, if it be viewed as a renal disorder we must search among those causes that operate more particularly on the kidneys. It has been attributed to over-work, diuretics; \&c. but without any apparent or receivable reason. 
To the surgeon I can imagine the treatment of a case of diabetes to be an affair of some degree of perplexity; wavering as his mind probably is about the uncertainty of its seat and nature, and having before him the animal diet system of Dr. Rollo, and the antispasmodic, tonic, astringent, and antiphlogistic plans of other accredited physicians, he has nought to resort to but the result of his own experience, or, if that be too limited, those impressions which his mind may have received from the public or private practice of another. This is a dilemma from which the veterinary surgeon however is in part relieved: animal diet he cannot for obvious reasons have recourse to; indeed, I do not think myself that he can to much advantage diet the animal at all; his views therefore may be exclusively directed to medical treatment. Ammonia may be made trial of, on the principle of its being one of the principal components of animal matter; sulphur and the sulphurets, as having a similar influence and being aperient; alkalies, calcareous and testaceous substances, to correct acidity; oak bark and forge water as tonics; \&c. \&c. but, for my own part, I would rather proceed on the antiphlogistic plan. I would bleed, but not largely, if the pulse indicated increased action, and repeat the operation if any benefit seemed to result from it. I would give mild purges, and in the intervals perhaps hydragyrus $\bar{c}$ cretá. I would also institute counter-irritation upon the loins; if not by blisters, by the mustard embrocation or some stimulating ointment that possesses no diuretic ingredient.

THE DISEASES OF THE BLADDER may be comprehended under the heads of cystitis, paralysis, and calculus. 


\section{Cystitis.}

Inflammation of the bladder may attack the peritoneal, muscular, or mucous coat, or it may confine its action to the neck. I know nothing about the first of these affections but as a concomitant or extension of peritonitis ; in which case, in addition to the regular set of symptoms, there will be shown to be present irritability of the bladder, by frequent small discharges of urine: this however will require no material variation in the remedial measures. And I cannot discover any important therapeutical advantage to be derived from drawing a line of distinction, even admitting it to be at all times practicable, between inflammation of the muscular and mucous coats; I shall therefore proceed to treat of these affections together.

The prominent symptom of either of these forms of cystitis will be frequent and painful efforts to stale, in which the animal will void his urine in very small quantities; for, observe, the irritability of the bladder may be exalted from two causes, either from an increased excitability to action in the muscular coat, or from the irritation to which the mucous coat is exposed from the cessation or alteration of its secretion; and both these causes have their foundation in inflammation. Febrile symptoms will keep pace with the virulence of the disease going on in the bladder. Without adverting to other affections, renal as well as vesical, however, this solitary local symptom will not bear us out in our diagnosis. The supposed disease in question may be nephritis; though we may suppress our suspicions on this head providing there be, in the aggregate, much urine voided, and that urine unaltered in quality; 
and providing the peculiarity of posture and gait and the tenderness about the loins be absent. Inflammation of the neck may also give rise to the same symptom; but from this it may at once be discriminated by ascertaining whether the bladder is full or empty : this may be done by an experienced hand by pressure before the pubes, but the more certain mode is examination per rectum.

This is rarely a spontaneous affection. Almost always we may detect some local cause of irritation, such as calculous matter or some other irritant in the urine. Professor CoLeman relates in his lectures the cases of two mares that were the property of Gen. BRow NRIG, in whom the mucous coat of the bladder was intensely inflamed, in one indeed mortified, with erosions through the viscus, in consequence of the introduction of some acrid caustic substance by the coachman, who, it appeared, was extremely desirous that the mares should go to horse: the death of one prior to the arrival of the Professor threw light on the nature of the other's sufferings; and she was recovered by active depletion and the copious injection of warm water into the bladder.

The treatment consists in taking away from four to six or eight quarts of blood; in administering from eight to ten drams of aloes; in promoting diaphoresis to the utmost of our power; in affording an abundant supply of plain water or some mucilaginous drink; in giving one or other of the alkalies to soften the urine; in instituting irritation before the pubes, but not by blisters, unless they be carefully washed off as soon as vesication takes place; in the frequent administration of enemæ until purging has begun; and, in the female, 
in injecting from time to time some soothing, mucilaginous, alkaline fluid into the bladder.

INFLAMMATION OF THE NECK OF THE IBLADDER will give rise to the same frequent and painful strains to stale as denote the last affection, and these efforts may also from time to time be productive of sparing discharges of urine; for it often happens that a little fluid will make its escape from the bladder in this state of preter-natural distention. This throws a cloud of obscurity over such cases in horses which, as is justly observed by Professor ColeMAN, the surgeon has seldom to cross his way; for the case of a man having this disease admits of solution in a great measure by ascertaining the space of time since he last voided his urine, whereas in the instance of the horse this is a piece of information we ean seldom obtain, or, if given, rarely place dependance on the report. In fact, as a general rule of practice, in all vesical affections we ought early to ascertain per rectum the state of the bladder, and not trust to this or to that account, or even, to the neglect of this indispensable duty, to the symptoms themselves however well-marked and decisive they may appear to be.

It seems that we are quite in the dark about the origin of this disease. It has been referred to spasm. All that we appear to know about it, is, that it is an affection that creates expressions of great suffering; but fortunately one of infrequent occurrence.

The first step to be taken, and that without delay, is to relieve the distended bladder, or the consequence may be its disruption. We are told we must pass the catheter. Most unquestionably this is a very desirable measure and would turn out to be a very effectual one 
should it prove to be practicable; but this I apprehend it will not be found to be in every case. What is to be done then? The bladder must be tapped. Now there are two ways of proceeding in this operation: one consists in carrying a trocar into the rectum and plunging it through the gut into the prominent bladder below; the other in thrusting the trocar through the recti muscles immediately in front of the pubes, whereby the bladder will be penetrated behind its peritoneal covering, and consequently the abdominal cavity left uninjured. Considering the horizontal position of the animal, and knowing the comparative facility with which these operations are performable, there exists no question in my mind which ought to be preferred; indeed, I doubt (for I never ventured upon the high operation) that the urine would be expelled through the rectum; whereas the dependance of the opening made in the front of the pubes singularly favors its discharge. Having once introduced the trocar I would endeavour to confine it there by a contrivance of tapes and rollers until I could succeed in passing the catheter. In the mean time, the same remedies as are recommended for the other forms of cystitis ought to be put in practice in this.

PASSING THE CATHETER in the male subject is not the same simple operation in a horse that it is in a man. In the latter, an inflexible instrument having a certain curve may with facility by a skilful surgeon be passed down the penis through the urethra into the bladder: not so in horses, in whom the urethra of the male makes two such sharp and sudden flexures under the ischial arch that all such attempts must prove abortive. To surmount this obstacle the way in which 
we proceed, is this-We first pass a whalebone or common bougie as far as the ischial arch, at which place its extremity may readily be felt in the perineum; we then make an incision, guided by the end of the bougie, so as to lay open the urethra to the extent of three-fourths of an inch ; through which artificial opening we introduce a female catheter and easily push it onward (the canal from that part being horizontal) into the bladder.

I have nothing to offer about PARALYSIS OF THE BLADDER; indeed, I know nothing of its existence but as an occasional consequence of disease or injury of the spine, to which part in course our attention must be directed to afford permanent relief.

\section{Calculus*。}

URINARY calculi, rather an infrequent formation in horses, are mostly found lodged within the pelves of

* Mr. Sewell did me the kindness to call my attention to the following detail in time for insertion as this Lecture was going through the press :-

"Wells, Somerset, August 9, 1824.

"Tire following account of the extraction of a stone from a horse's bladder has been communicated to me by Mr. W. MogFord, who was some years ago my pupil and assistant, and is now a respectable practitioner at North Low, near Oakhampton, Devon. Should you think it worth the notice of your readers, I will thank you to insert it in your valuable Journal. I am, \&c. James Whrse.

"The horse is the property of James Vear, Esq. near Hatherleigh, Devon. When taken up to be broke, he was found very restive, kicking off most of those who attempted to ride him : in consequence of this he received very rough usage, and has since been ridden rather hard. When Mr. Mogrord was desired to attend 
the kidneys: the reason of which is, as the Professot has long told us, the horizontal position of the trunk:

him, he observed a peculiar stiffiness in the murement of the hind legs, urine of a high colour and pungent smell, and a dribbling of urine from the penis for some time after staling: pulse between 70 and 80, and hard. By blecding freely, clysters, fomenting and embrocating the loins, and a week's rest, he appeared sufficicntly recovered to be sent to grass. He soon leaped over the gate of the field, and, crossing the country, got back to some pasture where he had been usually kept. This exertion caused a return of his complaint, and Mr. Mr. was again desired to attend him. He found him in the same state as before described. Wishing to examine the bladder, he introduced his hand into the rectum for that purpose, and immediately felt a hard substance, which appeared to him to be a stone in the bladder. He communicaterl the circumstance to Mr. Fisher, surgeon, of IIatherleigh, who could not be persuaded that it was a stone, until he had made the examination himself, when he also felt it distinctly. Mr. M. then proceeded to the operation in the following manner:-

"Having drawn out the penis from the sheath or prepuce, he passed a rod of whalebone up the urethra, until the end of it could be felt in the perineum. He then cut down upon the end of the rod, and through the opening thus made in the urethra he introduced a director, and with a probe-pointed bistoury continued the opening as far as the left side of the anus. He then introduced his right hard into the rectum, and the two forc-fingers of his left hand into the bladder, and, without any difficulty, pushed the stone against the middle finger, by which he guided it to the neck of the bladder, and then easily forced it out through the opening in the urethra. The stone weighed rather more than four and a half ounces. Some parts of the stone appeared to have been broken off, and left in the bladder : these were easily removed by means of a bit of soft sponge tied to a whalebone probe, and some warm water. The wound quickly healed, except a small orifice, through which a part of the urine still passes; but the horse has worlsed hard since, and suffered no inconvenience from it. MI. M. has no doubt that a stone of seven or eight ounces might be thus extracted,"-Med. and Phy. Journal. 
did the ureters descend as they do in man there can be no doubt but that most of the stones would be found in the bladder; as it is, calculus in the biatder is a rare occurrence. LArosse, in his "Dictionaire d'Hippiatrique," gives an instance of stonc in the bladder which occasioned pain and trouble in staling, and frequent urinary discharges; this led to an examination per rectum, and the stone was felt, and afterwards extracted by an operation similar to the one parctised in human surgery. I have never had an opportunity of operating myself; nor am I in possession of the particulars of any recent operation of the kind. Many years ago the operation was about to be undertaken by my father at Woolwich; but the horse was suddenly seized with convulsions and died in the interim of suspense. The stone was found within the cervix, ulceration had made holes through the bladder, and two pails-ful of urinous fluid were measured out of the abdomen which had created intense inflammation of the peritoneum.

An unobjectionable and ready mode of ascertaining the presence of stone is, in my opinion, manual examination per rectum while the animal is thrown upon his back, this being the position in which the calculus will in the male impress the gut: in the female, the vagina is the channel of introduction. Were I however called to a case of this description I should not proceed by "cutting for the stone;" I should give the decided preference to the operation of DILATATION, one that, it strikes me, with an instrument properly constructed, is singularly applicable, not only in the female but in the male subject, to veterinary practice. 


\section{LECIURE LVI.}

\section{On the Male Organs of Generation.}

THE male animal, though his part in the business of procreation appears to be but simple when compared with the tedious and critical process of generation in the female, is nevertheless provided with an assemblage of organs whose individual structures and functions we may rank among the most complicated in the system. These organs consist of glandular bodies whose office it is to prepare the fluid necessary to impregnationthe testes; of canals and receptacles to conduct and contain the prolific fluid-the vasa deferentia and vesicule seminales; and of an instrument to transmit this fluid into parts formed to receive and dispose of it in the female-the penis and its appertenances.

\section{The Testes.}

The two testicles, the truly essential organs of procreation in the male, hang between the thighs within a bag called the scrotum: a part I shall commence my description with.

THE SCROTUM or purse consists of a loose production of the common integuments, laterally continuous 
with the skin covering the flanks and thighs, in from with the sheath of the penis, and behind with the perineum: a term under which may be comprehended the space between the scrotum and anus. The skin forming the scrotum is thin and soft in its texture, generally black, and is clothed with fine downy hair, long and bushy around its sides, short, scanty, and hardly perceptible about its inferior parts. The prominences of the testicles produce a longitudinal crease in the middle of the scrotum, named the raphe, of which a faint trace extends into the perineum: this crease denotes the line of attachment of the septum scroti. Prior to the appearance of the testicles the purse is comparatively small and insignificant, consisting merely of some loose folds of skin ; but during their descent from the belly it becomes gradually developed, and the wrinkles from extension by degrees disappear.

On cutting through the integuments of the scrotum we expose a pale, yellowish, fibrous layer of substance which is considered to be muscular; indeed by some it is described as a distinct muscle and named the dartos; by others it is regarded but as a continuation of the faschia superficialis abdominis. Its fibres, which run longitudinally, are strongest where they cover the testes; they are extended anteriorly into the cellular substance of the sheath, and posteriorly are continued upon the root of the penis. This substance loosely adheres to the skin by cellular membrane, and is still more loosely connected by similar interposition to the parts within. It is certainly distinctly fibrous in composition, but its fibres possess a density and toughness and a yellow cast which accord more with the pro-

PAR'T III. 
perties of ligament than muscle; and furthermore, I am acquainted with no physiological fact that warrants such an inference, for the scrotum of the horse does not corrugate from the application of cold or other stimuli as that of a man is known to do; we cannot therefore avail ourselves of the contractile power of the scrotum, at least not to the same extent as some human anatomists have done, to strengthen suppositions of the presence of a dartos. This fibrous layer is most loose and abundant along the middle of the bag, where its expansions from either side unite and are reflected upward in inseparable union with each other in the space between the testes, forming in this manner the septum scroti, which consequently extends from the raphe to the under and posterior part of the penis, and serves to prevent one testis from encroaching upon or interfering with the other.

The cellular membrane of the scrotum, which may be considered as the third layer of substance entering into its composition, is interposed in the greatest abundance between the fibrous expansion and the peritoneal coverings of the testicles. Being very long and loose in its texture, and destitute of any adipose matter, the parts it connects are extremely moveable upon each other and admit of considerable extension.

Continued from the faschia superficialis abdominis upon the spermatic cord, and thence into the fibrous texture of the scrotum (which, as I remarked before, has been regarded as a prolongation of it) is a thin, cellular, and fibrous expansion; and underneath this runs a layer of fleshy substance that has, when contrasted with the surrounding white parts, a remarkably red aspect: this is the CREMASTER muscle. Its 
Heshy fasciculi have a tendinous origin from the internal oblique and transversalis muscles at the internal ring, whence they pass obliquely backward upon the cord, are reflected forward and inward with it through the external ring, and end slantwise in a digitated margin, just as the cord joins the testicle; from these digitations several slender flat tendons descend and expand upon the inferior surface of the tunica vaginalis, with the substance of which they become so interlaced that we lose all further trace of them. These tendons add greatly to the firmness and resistance of the vaginal tunic, rendering it more efficient as an external covering and protection to the testicle. The muscle itself aids in the suspension of the testis, and in action will retract it as high as the ring: it is more especially called into action at the time of coition -at the moment probably that semen is passing from the organ into the urethra. This is a comparatively feeble muscle in the human subject, and is commonly an involuntary one; though there are persons that can draw their testicles up at will. In order to give some notion of the forcible action possessed by the cremaster in the horse, I would simply desire those who are in the practice of castration to reflect on the force required at the moment that the testicle is graspèd by the hand to counteract its retraction. When the testes hang low, and feel loose and pendulous within the purse, which is stretched and rendered thin and flaccid by their weight, it is according to a vulgar notion a sign of debility: the parts are found in this condition after the act of coition, and this circumstance probably has given rise to the above supposition; and so far reasonably enough, for then the cremasters become relaxed 
both from the fatigue consequent upon their long-continued and forcible contraction, and from a general sense of debility or dullness which is known after the act to take temporary possession of the system altogether.

The first or external covering of the testes, one common to it and the cord, is a borrowed one from the peritoneum, commonly known as the tunica vaginalis. The acquisition and reflection of this covering will not be clearly understood until the descent of the testicle has been explained; it may just be observed here, that it is a continuation of peritoneum formed into a loose vagina or sheath, which begins at the internal ring, proceeds with and envelopes the cord, and, lastly, covers in the same loose manner the testis and epididymis; so that if the bag be punctured and air or liquid be impelled into the cavity, the fluid will readily distend it and mount into the abdomen, inasmuch as both cavities have a free communication at the internal ring. The vagina however does not end in single investment of the testis; along the superior and anterior border of the epididymis we find it fixed but not terminated; for here it becomes reflected, first upon the epididymis, then upon the testis, and lastly upon the cord, so as to give them all a second covering, which only differs from the first in being everywhere in close adhesion with the parts it invests: this last production is called by way of distinction the tunica reflexa or tunica vaginalis TESTIS ; it must not be forgotten however that they are both peritoneal productions-one and the same continuous membrane. The vaginal cavity has a smooth polished surface, and this is constantly bedewed with a limpid, colorless, serous 
fluid, which in the operation for castration spirts out the instant the knife or cautery has penetrated the tunica vaginalis : it is an accumulation of this fluid that constitutes hydrocele, a disease that has no existence, I believe, in the horse abstractedly from abdominal dropsy.

THe TESTES, with their appendages, the epididymes, constitute the apparatus secretory of the male sperm or semen : the penis, as I stated before, being simply the instrument of transmission. We have already seen that these bodies, though pendulous, are not loose within the cavity of the scrotum; we have found them enclosed in peritoneal tunics, confined by cellular and fibrous envelopes, and fenced in their respective apartments by the septum scroti.

The general figure of the testis is that of an oval flattened at the sides; the magnitude, that of the egg of a hen: M. Girard says, that the left is generally a little larger and more pendent than the right. The testis is suspended within the scrotum by the spermatic cord; and in such a position that its long axis corresponds to that of the body; it has therefore anterior and posterior ends; to the latter is joined the termination of the cord, while the vas deferens departs from the globus major of the epididymis which is attached by a ligamentous band to the former: it has also an inferior or convex border, along which are seen the tortuous windings of the spermatic artery; $a$ superior or straight border to which is attached the epididymis; and two convex sides free and unconnected.

The spermatic cord, the substance through which the testicle is connected with the cavity of the abdomen and by which it is suspended within its scrotal 
apartment, is composed in the following manner:1st. It has four coverings; there is immediately under the skin the faschia superficialis; next, the cremaster; thirdly, the tunica vaginalis; and lastly, the tunica vaginalis reflexa: within the cavity formed by the vaginal tunics it is that the intestine is protruded in inguinal and scrotal herniæ; the hernial coverings, consequently, exclusive of the sac, are the faschia and the cremaster muscle. 2ndly. The component parts of the cord are-a. The ARTERIES which are two in number: the artery of the cord, a small branch of the external iliac, which ramifies and expends itself upon the cord; and the spermatic artery which, having arrived at the internal ring, enters the inguinal canal, runs within the posterior part of the cord, growing tortuous as it descends, serpentines along the superior border of the testis, between it and the epididymis, winds round the anterior end of the gland, and lastly reaches the convex border where it becomes extremely convoluted and where its branches are principally distributed. In its descent it detaches small unimportanit twigs to the adjacent parts; and as it approaches the testicle becomes surrounded by an assemblage of venous vessels. $b$. The veiNs, comparatively numerous, accompany their correspondent arteries; the spermatic veins indeed nay be said to make up the principal bulk of the cord, for they are not only numerous, but large and flexuous, and as they approach the testis form a sort of plexus which has got the name of corpus pampiniforme: these return their blood into the posterior vena cava. c. The NERVES, which are derived from the hypogastric plexus, also accompany the spermatic artery; they are small but sufficiently numerous: 
though the testis does not possess great sensibility in health, we may vouch for its being acutely sensitive in a state of disease. $d$. A Bsorbents are both large and numerous in the cord. They are readily found alongside of the venous trunks; and not infrequently may be filled by introducing mercury into the spermatic artery. $e$. The VAS DEFERENS, though commonly considered as a constituent of the cord, it must be remarked, takes at first a solitary course, remote from the blood-vessels; seeing that it springs from the summit of the head of the epididymis, which part surmounts the fore-end of the testicle, in order to go the nearest way to the cord, its course, which begins with a series of convolutions gradually unwinding as it proceeds, becomes necessarily oblique nearly as high as the external ring, where it joins the blood-vessels, and continues to accompany them posteriorly through the inguinal canal; at the internal ring it leaves them, turns inward and ascends into the pelvis, where we find it creeping along the side of the bladder infolded in peritoneum to get to the cervix, crossing under in its course first the umbilical artery and then the ureter; at length it terminates by rather a contracted orifice within the mouth of the duct of the vesicula seminalis, just behind a little eminence in the urethra - the caput galinaginis, about an inch posteriorly to the cervix of the bladder. Within the inguinal passage this duct is accompanied by the artery of the vas deferens, a long slender branch of the epigastric. Its canal, flexuous until the duct has joined the cord, but straight in its subsequent course, is not uniform throughout in calibre; the area of its tortuous part is large, but as it becomes straight it grows contracted; 
having entered the pelvis it gradually enlarges again, and acquires unusual volume in running along the side of the bladder; and the canal of the enlarged portion presents a reticulated structure, which gives its exterior an irregular, tuberculated appearance; the most contracted part is that in union with the duct of the vesicula seminalis, which is a comparatively small cylindrical conduit. The parietes of this duct are so remarkably thick and firm to the feel that we distinguish it at once by the fingers from the other parts of the cord: they consist of two tunics; the external one, in which its thickness consists, is white, fibrous, and approaches in appearance to cartilage; the internal one is thin and fine in texture, muco-membranous in its nature, and here and there incloses a reticulated structure. The different constituent parts of the cord are connected altogether by cellular substance destitute of fat; and from the circumstance of the parts in general being more bulky below the ring the cord increases in breadth and thickness as it approaches the testicle.

The close or intimate tunic of the testis is perfectly distinct from its vaginal coats, and from its conspicuous whiteness is called the tunica albuginea. Though it may be stripped off in places by the forceps, and the separation may be also affected by diffusing quicksilver into the cellular texture uniting them, the tunica reflexa so intimately and uniformly adheres to this tunic, that, the former being transparent, they appear to constitute but one and the same covering. The albuginea however is much denser and firmer in its nature, and much resembles the other fibrous membranes, particularly the dura mater, both in appearance and texture: like that membrane it is extensile and 
contractile, but neither suddenly nor greatly so, and imparts a compactness and firmness of feel to the inclosed structure which the latter, deprived of it, is not found to possess. Over the convex part of the gland this tunic is perforated by numerous holes for the transmission of the spermatic blood-vessels; internally it is connected by these vessels to the substance of the testis, also by a fine cellular web, and by numerous little processes that traverse the gland perpendicularly, serving to hold the internal parts together as well as aiding to preserve the form of the whole, and which are generally described as the septa or septulce testis.

On cutting into the testicle we find that it consists of a soft pulpy substance of a pale brown color, and that this by being nipped and drawn out by the forceps is extensible into numerous whitish threads of extreme exility which may occasionally be elongated to a considerable degree: it is believed that these are the seminiferous tubes. Like other secreting organs, the testis receives into its composition congeries of arteries, veins, nerves, and absorbents, the peculiarity of structure chiefly residing in the arrangement of the secretory and excretory parts of the apparatus; it is imagined, in the instance before us, that this consists in many vascular coils or complexures which are only separated from one another by the septulæ, and that these, independently one of the other, possess the power of secretion. After the spermatic trunk has dispersed its ramifications over the albuginea, numerous twigs are transmitted through that tunic into the interior of the gland: from these, capillary arteries spring, which, it is presumed, communicate wit's the tubuli seminiferi, 
but in what manner anatomists have not been able to detect; for in the old subject these tubes are constantly plugged with secretion and so foil those who make attempts to inject them, and in the young subject, before they begin to secrete, they are not sufficiently developed to admit of the examination. If quicksilver be suffered to pervade the arteries by its own weight it is found to return by the veins ; and in a preparation of the testicle of a dog at present before me the absorbents have been filled from the same source. Within the posterior end of the testis the seminiferous tubes assemble from the different parts of the interior and unite into a set of larger tubes of the same description, which are disposed after the manner of network, and hence have got the name of the rete; then, from the rete proceed another set of similar tubes still larger, about a dozen, I believe, in number, from the testis to the epididymis, constituting the sole medium of communication and the principal one of connection between the two, which are the vasa efferentia. In addition to these minutiæ, we may notice that the superior border of the testis is marked by a broad white line; this denotes the situation of a supposed canal, and is generally mentioned as the corpus Highmorianum.

The EPIDIDYMIS is extended along the superior border of the testicle, upon which it rests, and to which it is connected by the tunica vaginalis reflexa. Its ends are bulky in comparison to its middle : that receiving the vasa efferentia, the smaller one, is the caput or globus minor; the other, giving rise to the vas diferens, is the globus major - the part farriers call the nut. The interior of this appendage to the testicle exhibits a structure entirely vascular the vasa efferentia unite 
and re-unite until they form a single duct of whose numberless and very remarkable convolutions the globus major is entirely constitued: these tortuosities, which, when squeezed, freely emit semen, will admit of being unwound for a considerable extent so as to have the length of the duct calculated with very tolerable exactness from beginning to end, which has been found to amount to several yards. It is small at its formation, but grows imperceptibly larger in making its endless windings and turnings until at length it assumes the size of the vas deferens, in which it ends. Its various convolutions are connected together by cellular membrane, and are interspersed with a sparing supply of blood-vessels.

The course of the sernen then is this-It is secreted by the capillary coils of the spermatic artery, from which it is received by the tubuli seminiferi; these tubes convey it into the rete, and the rete discharges it through the vasa efferentia into the epididymis, from which it is conducted by the vas deferens into the urethra.

It is a singular fact, that the organs whose structure we have been investigating are originally formed in a situation remote from that in which they are destined to carry on their functions : "the colt has no testicles," is the common observation of the uninformed on these matters, and we know ourselves that the purse is without them, but we also know that they exist ready-formed within the abdomen, and that they will descend at a certain period of age into their proper receptacle, the scrotum. During the fotal state we find them more or less developed, tinged with a blush of red, lodged underneath the psor muscles, in contact with the in- 
ferior borders of the kidneys, covered and retained in their situations by peritoneum, and concealed by the intestines around them. Here the organ receives its arteries from the contiguous trunk-the posterior aorta; the vas deferens runs forward to meet it ; and the cremaster likewise turns forward instead of backward: there being at this time no such thing as a spermatic cord. Thus placed, the testicle may be regarded as one of the glands of the abdomen; indeed, it has considerable similarity to the kidneys in receiving its vessels from the same contiguous source, and in sending a long duct backward into the cavity of the pelvis; and there does not appear to be any conclusive reason why it should not perform the same office in that situation that it does in the scrotum, and particularly since it is known that in birds they remain and secrete there during life. From the part where the blood-vessels enter we find growing a whitish substance which is extended backward, diminishing in breadth as it recedes, through the ring, where the fibres of the cremaster may be traced upon it, and whence it is prolonged into the scrotum, growing still narrower until it vanishes: this substance, regarded by some simply as a ligament, was considered by Mr. HUNTER, as the gubernaculum or pilot by means of which the testis is directed in its passage from the cavity of the abdomen into that of the scrotum. Quitting the spot where it has been formed and matured, the testicle gradually retrocedes, guided by the gubernaculum, until it arrives at the internal ring, which, at this time (like every other part of the parietes) is closed by peritoneum; this temporary obstruction the testis overcomes by drawing the membrane with it through the ring and carrying the pouch made 
thereby down into the scrotum; the gubernaculum at the time undergoing a complete inversion. This accounts then for the production of the tunica vaginalis, and explains how that membrane comes to be doubled or reflected; for the testis receiving originally (as an abdominal viscus) a close and adherent peritoneal tunic, and acquiring another, which forms a loose covering, as it passes through the ring, must necessarily have two; and since both are derived from one and the same membrane, it follows that one must be a continuation of the other. These elongations of membrane, though in most places in constant contact, are prevented from adhering together by a continual exhalation of their natural serous secretion; any interval that may subsist between them in course communicates with the cavity of the abdomen through the ring, a part that remains open through life: this however is not the case with man; in his body the communication is cut off, after the testicles have descended, by a natural contraction and obliteration both of the ring and the inguinal passage. In many instances one, in some few both, of the testicles are known to have remained within the belly through life. As we are unacquainted with the immediate cause of their descent, so we are unable to give any rational explanation of this phenomenon. I have understood, that in many of these cases the glands have been found to be imperfectly developed : this however is not without exception.

Man and many other animals have their testicles within the scrotum at the period of birth : in the human fœetus they begin to move about the seventh month ; about the eighth they reach the groins; and before birth arrive in the scrotum. But, in the horse, they 
do not pass through the ring until the sixth or seventh month after birth, and are seldom let completely down into the scrotum before the expiration of the twelvemonth. In some cases, one testicle will not make its appearance for a considerable time after the other; and as the operation for castration is seldom long delayed after the first year, this will account for the rigs (as horses having but one testicle are called) with which we meet every now and then. Again, instances are not wanting in which one testicle has descended to the ring and there remained through life.

\section{The Vesicula Seminales}

Are two, oblong, membranous sacs, placed contiguously to the terminations of the vasa deferentia, which have been so denominated from a supposition that they were receptacles for the semen. They occupy the lateral intervals left between the bladder and the rectum with their internal prominences, while their external are opposed to the sides of the pelvis; they are principally sustained in their places by the bladder : in relation to each other they represent in situ the two sides of a triangle, for their posterior extremities are nearly in contact, while their bases diverge as they advance and leave a considerable breadth of interspace. The longitudinal channel between the bladder and vesicula is occupied by the vas deferens. The vesicula is confined in its situation by cellular connexions with the bladder and rectum, the walls of the pelvis, and the vas deferens.

These bodies have a near approach to the pyriform: anteriorly they present broad round bases which are elongated posteriorly into contracted circular necks, 
and the necks end in ducts of some length which may (to carry the resemblance on) be said to represent the stalks of the pears; they incline however to convexity superiorly and to flatness inferiorly, and their surfaces are rendered uneven by some tubercular eminences. The parietes of the vesicula are distinguishable into two textures. The external one, when cleared of the enveloping cellular substance, is white, and, though soft to the feel and not so thick as the outer tunic of the vas deferens, possesses considerable density and toughness, and in some parts, particularly around the base, assumes a fibrous texture: these fibres are described as muscular, and certainly the functions attributed to the vesiculæ appear to demand such a supposition were we unconvinced of its truth from anatomical inspection, which, I should say, we are. This tunic is lined with a membrane, whose surface is of the papillary description and everywhere presents to view (through a magnifying glass more plainly) numerous pinhole-like perforations which are the orifices of subjacent follicles furnishing the whitish, viscous, jelly-like secretion peculiar to these bodies. Their ducts are of considerable volume, not greatly inferior even to the urethra itself : they proceed backward in a convergent direction in union with the vasa deferentia, alongside of the membranous part of the urethra, and terminate behind the verumontanum. 


\section{LECTURE LVII.}

Penis.

THE penis or yard is an organ of large dimensions in the horse species, capable of projection and retraction, ordinarily concealed within a loose and pendulous doubling of skin at the inferior and posterior part of the belly. It is not only destined for the important office of impregnation, and made the seat of that enjoyment experienced by the male during the copulative act; but it serves the subordinate purpose of a convenient conduit for the discharge of urine.

THE SHEATH in which the penis is inclosed, and by which, unless it be in a state towards erection, it is concealed from view, is nothing more than a prolongation of the common integuments, having one part withdrawn within the other and puckered so as to form a sort of corrugated bag, corresponding to the prepuce in man, whose dimensions vary with the degree of retraction of the peris. Posteriorly the sheath is continuous with the scrotum; anteriorly it is cleft and has two large pendent folds of skin, proceeding from the sides of the vagina propria penis, with a broad deep furrow between them, and extending along the belly as 
far forward as the navel. In must horses these parts are dark-colored or black; in some they are clothed with fine, long, downy hair; in others they are inferiorly almost bare, having but a few scattered short hairs which are only perceptible on close inspection. But there is so much variety in all these particulars that any single description accurately and minutely drawn will not apply with precision to another individual. Near the borders of reflection of the sides of the sheath, in the ordinary state of the parts, grow two small papilla or teats resembling the dugs of the mare; they present, in a less perfect degree, the same internal structure and are perforate at their apices: they are not to be found however, I believe, in all horses, and it is the practice of some cutters to take them off at the time of castration; GIRARD observes with truth, that they are largest in the ass species. Internally, the sheath - the vagina propria penis, exhibits two large, circular, tegumental plications : the outer and larger one is formed by an involution of the external vagina, and exhibits internally numerous corrugations, which, though by no means regularly ranged, for the most part run in circles around the inner plication, the veritable preputium glandis penis, whose internal surface is also puckered into circular corrugations, but smaller, and still more numerous, and more irregularly disposed than those of the former. These plications are nothing but a continuation of the common integuments disposed after this mamner to admit of the projection and erection of the penis, a state in which they, together with their corrugations, are extended and annihilated; the skin composing them however is of a kind remarkable for its

PART III. 
fineness and softness of texture, for its black or marbled complexion, and for the excretory pores of myriads of little subjacent glands which are so closely packed that it is hardly possible to introduce the point of the scalpel through it without penetrating one or more of them. These involutions of skin are supported in their corrugated form and connected to the body of the penis by an interposed stratum of cellular substance which is so loose and abundant as to readily admit of their sliding over each other, and of the penis being protracted and retracted with the greatest facility.

From the inner plication the skin is reflected upon the penis, extending forward and giving a complete covering to the part named the glans penis. This part exhibits a very remarkable corrugated aspect which I cannot resemble to any thing so nearly as to the leaf of a curled cabbage : its cellular substratum is shorter and more condensed than that of the sheath, it not being designed to be protracted beyond the penis ; and like the inner plication it is destitute of those glands with which the outer is so thickly studded.

The glandula odorifere are those numberless little brown follicular bodies that are set in the internal part of the outer vaginal fold, and discharge a white caseous matter through their excretory pores upon the surface. This secretion, which has a peculiar odor, preserves the sensibility of the parts and facilitates their slipping backward and forward ; it is occasionally found collected in considerable masses between the outer and inner plications, even, it has been said, so as to plug the orifice of the urethra and cause retention of urine: knowing this, will put us upon our guard, and lead us 
to inspect the part in cases where the cause of retention is not apparent. The surfaces covered by the inner plication are lubricated by a mucous secretion.

There is yet another involution of the skin. This takes place at the extremity of the organ, where a little cul-de-sac is formed, in the centre of which protrudes the end of the urethra, a part also covered by a reflexion of this thin, hairless, corrugated integument. Around the end of the urethra is a little recess partially divided into two chambers, commonly containing concreted masses of a peculiar unctuous secretion: the use of this excavation is not very apparent.

The penis inferiorly receives a covering from the faschia superficialis abdominis, lateral portions of which descend from the flanks and unite along its middle : as we approach its root the faschia grows stronger, and exhibits a fibrous texture. The organ derives considerable support from this covering.

THE PENIS is constituted of five distinct parts, which appear to view without the aid of dissection or disarrangement: viz. the two corpora cavernosa, which form the middle and sides, the largest proportion of the organ; the glans penis or head, the anterior protuberance; the corpus musculosum urethre, making up the inferior portion; and the plexus venosus, surmounting the corpora cavernosa. But, before I proceed to describe these several parts, I shall point out the muscles belonging to the organ.

Erectores penis. A pair of short, thick, fleshy muscles covering the roots of the penis and the sides of the perineum. They arise by broad attachments from the inferior and posterior parts of the tuberosities 
of the ischia; mostly fleshy in sulustance, but blended with a thin expansion of tendon superiorly, they take a convergent course, upward and forward, enveluping the crura penis into which they are extensively inserted. Their office is to promote the influx of blood into the organ at the time that it is erecting; and when in that state more completely to distend its body by pressing still more blood into it from the crura.

Triangularis PENis is a thin, triangular, fleshy production, spread upon the upper surfaces of the small prostrates, extended from them to the large prostrate to which it is attached, and surrounding the intermediate or membranous portion of the urethra. Its fibres, which are palish, run across the botton of the cavity of the pelvis above the ischial arch from one ischium to the other, and have loose attachments to those bones through the intervention of the cellular substance in which they are invested. It will contract the membranous part of the urethra in action, and have probably some effect in discharging fluids from the prostrates.

Accelerator URIN瓜. This muscle is incorporated with and may be considered as forming a part of the penis itself. It springs from the middle and posterior part of the bulb of the urethra, where it is joined by some scattered fasciculi coming on each side of that body from the perineum. It takes its course along the under part of the penis, and may be followed for some short way upon the glans, forming throughout its extent a complete fleshy covering to the urethra. It will aid in the forcible expulsion of the urine; but it is principally exerted, I imagine, in the ejection of the semen. 
TIIE CORPORA CAVERTOSA PENIS, the principal parts in respect to bulk in the constitution of the organ, parts whose erectile capacity fits it as the instrument of copulation, extend from the ischial arch to the glans penis, insinuating their extremities for some little way under the flattened portion of the latter. They are attached by means of two branches, the crura penis, to the posterior ends of the tuberosities of the ischia, and to the sides of the ischial arch, where they are clothed by the erectores penis; from these attachments they converge along the branches of the ischimm and under the summit of the ischial arch form a union. The attachment of the penis to the pelvis is considerably strengthened just below this union of the crura by two suspen. sory ligaments which fasten the sides of the cavernous bodies to the pubes. These cylindrical bodies, then, conjoined in one common tunic, continue their passage forward to the glans penis within the substance of which they terminate by two rounded protuberances.

The corpora cavernosa are composed of a ligamentous sheath of unusual thickness and toughness, common to them both, whose fibres, running in every direction, are so interlaced and matted together that it not only defies our industry. to unravel it, but is even impenetrable unless when sharp instruments, and they with no ordinary force, are made use of. It is not everywhere uniform in thickness however: it is comparatively thin over the crura and where it is opposed to the urethra, those parts being sufficiently supported and protected by their muscular coverings. 'Their internal structure, spongy, reticular, or honeycomb-like in its arrangement, is found to involve three different textures. First, numberless little tendinous cords, which are processes from the ligancntous sheath, un- 
equal in size, run irregularly from one side of the interior to the other: these are crossed in the middle by a perpendicular set which are broader and stronger, and so arranged as to resemble the teeth of a comb; hence these last, which form the partition between the corpora cavernosa, have been collectively named the pecten. Thus, preternatural distention of the organ, and consequent liability to rupture, are guarded against both laterally and perpendicularly. Secondly, the interspaces are filled with a pale-red spongy substance in which may be distinguished cylindrical processes taking a longitudinal course: these M. Gira RD pronounces to be "évidemment musculaires;" and I see reason for so considering them, though I think it is a case in which we ought to seek confirmation of the fact from physiological or experimental inquiry and not entirely rely upon anatomical evidence. Thirdly, these cylindrical bands are surrounded and connected together by a cellular structure that would appear to result from some peculiar venous arrangement : at least, the small veins, which are here far more numerous than the arteries, have everywhere the freest communication with these apparent cells.

The cavernous structure is especially supplied with blood by a large vesisel, the internal pudic artery, a branch of the obturator artery, which enters the crus, giving off several small branches to the other parts of the organ. The pudic nerves, two in number, springing from under the ischial arch, are seen entering the corpus at the same place, one before, the other behind the artery. The internal pudic veins, which are large and numerous, divide here into two sets, one accompanying the arteries, the other the nerves, after they have quitted the penis. 
THE GLANS PENIS is the large, irregular, fungous protuberance, forming the anterior part of the organ, which is vulgarly called its head. This part puts on a very different appearance when distended from the state in which we find it in the dead animal: it then presents a broad surface anteriorly surrounded by a prominent inflected border, which is turned back for some distance above, but altogether deficient below, where there is a notch or division of the glans. The lateral portions are prominent, incorporated superiorly, separate and divergent inferiorly: posteriorly they are terminated by a contracted circular part, whose prominent border is the corona glandis. From the corona extends along the dorsum penis a flattened substance of an oblong oval figure, which is the posterior or terminating portion of the glans. In the middle of its anterior surface is a prominence that marks the termination of the corpora cavernosa; and below this is a circular fossa having in its centre the projecting orifice of the urethra. Altogether, the glans may be regarded as a part by which the penis is surmounted to enable the animal to direct the semen with certainty into the womb of the female, whose mouth is precisely its counterpart in form.

The internal corrugated production of sheath and the reflected portion by which the glans is immediately covered have already been described: underneath this last we find a fibrous case, of a ligamentary and cellular texture, holding together the internal structure. The interior exhibits a honeycomb appearance, is throughout composed of a soft spongy tissue, in a high degree distensible and elastic, which some are of opinion is nothing more than a congeries of veins: whether this 
be wholly or in part true, venous vesscls are found among the cells, and become large and more conspicuous towards the posterior portion, from which spring those veins that afterwards so suddenly enlarge and multiply upon the dorsum penis, forming there that remarkable, convoluted, venous assemblage, the

Plexus venosus penis. A structure that may be considered probably as a development of the glans; and one that, when distended with blood, in the erect state of the organ, constitutes no inconsiderable part of its volume. Though the veins composing it have free and frequent communication, yet, being furnished with valves, this communication is not such as will permit us to fill them with common injection contrary to the course of the blood: so that when we mean to distend them with wax we introduce the pipe into the substance of the glans. Towards the root of the penis these venous convolutions diminish in number and volume, and at length end in front of the symphysis pubis in three or four veins of ordinary size, which are here joined by the epigastric and superficial femoral veins and then proceed into the pelvis to arrive at the trunk of the internal iliac vein.

THE URETHRA is a membranous canal extending from the bladder to the extremity of the penis and affording a passage for the urine and seminal fluid. Arising from the neck of the bladder, the urethra, in its way to the outlet of the pelvis, runs at first horizontally backward with a slight curve downward between the lobes of the great prostrate, embraced superiorly by the portio media of that body, and arrives at the small prostrates, which are situated upon its sides: this intermediate portion is called the membrunous part; 
incorrectly however, for we find that it is encircled by some of the fibres of the triangularis penis. Behind the small prostrates it suddenly bulges or swells in volume, which part is named the bulb: it is here that the accelerator urinæ begins, the muscle that incloses the canal during the remainder of its course, and is in fact a part itself of the urethra. From the bulb the urethra suddenly curves downward and shortly after sharply forward, passing under the ischial arch, the tuberosities bounding it laterally, at which place it is joined by the crura and subsequently surmounted by the corpora cavernosa. At the extremity of the organ it is surrounded by the glans, and terminates in the centre of the anterior part of that body in a prolapsus of membrane covered with a reflection of fine skin by an open orifice which is insulated from the glans by the fossa running round it. In turning round the ischial arch the urethra has extensive cellular attachments to the bone; but it is principally retained at its curvature by the suspensory ligaments of the penis. The length of the urethra is nearly two feet, and it is a canal of considerable dimensions, so that I can with facility introduce the handle of the scalpel into the orifice, though that is the smallest part; for, after it has passed the bulb, it pretty regularly diminishes in calibre all the way to the orifice: the canal at the curvature measuring, when distended with wax, four inches in circumference; near the orifice, not more than two.

The interior of the canal exhibits a smooth, polished, lubricated surface, having a pale blush; in fact, it does not differ from that of any other mucous membrane, unless it be that its mucous follicles are larger and more numerous, whose orifices are here called lacunce. 
It is continuous, and appears to be one and the same texture, with the mucous membrane of the bladder. About an inch and a half from the neck of that viscus, within the membranous part of the canal, is a little eminence named the verumontanum or caput galinaginis, at whose root open the ejaculatory ducts: the use of this tubercle (which I believe has not been noticed) appears to me to be, to perform the office of a valve to the orifices of these ducts, preventing any influx of the urethral fluids. Upon either side of it are many small perforations through the membrane: these are the excretory openings of the ducts of the great prostrate. A little in the rear of the verumontanum, are several, small, papillary eminences, perforated through their centres, and longitudinally ranged in two distinct sets: these are the openings through which the ducts of the lesser prostrates discharge their secretion.

The bulb of the urethra is a longitudinal prominence beginning immediately behind the lesser prostrates and proceeding with the canal around its curvature; not indeed ending there, for it may be traced on, considerably shrunk in volume, even to the glans penis: the continuation of it being the part corresponding to the corpus spongiosum in human anatomy, a name it hardly merits in this instance. It has a cellular arrangement internally which we may regard in the same light as the spongy structure of the glans itself : indeed, if we might be allowed here to draw a comparison between these parts in a man and in a horse, the spongy body being more developed in the penis of the former, the glans in that of the latter, we should say that the glans was an expansion of the corpus spongiosum, and that the glans in the horse and the corpus 
spongiosum in man bore strong evidence of some peculiar venous arrangement.

\section{The Prostrate Glands.}

THESE bodies are three in number: a single large one situated at the neck of the bladder corresponding to the gland of the same name in the human subject; and two small ones which answer in situation to two little glandular bodies occasionally met with in man, named from their discoverer Cowsper's glands.

The great Prostrate embraces the neck of the bladder and incipient portion of the urethra, both superiorly and laterally, being placed between those parts and the intestinum rectum; consequently it is supported by the two former. It is firmly bound to these parts by cellular adhesion; and has also a cellular connection in front with the vesiculæ seminales, and above with the rectum. We distinguish in this gland two lateral lobes and a uniting middle portion, which in man Sir E. Houe has described as the third lobe, in consequence of its assuming frequently the lobular form when in a state of disease. The lobes are enveloped in incompact cellular cases, have exteriorly a conglomerate disposition, and are of a palish brown color. Internally, this body possesses a spongy or cellular structure, diffused through which are sets of thin membranous tubes, very large for the size of it, which are readily inflated, and then are seen to open, through orifices before described, around the base of the verumontanum. The gland secretes a whitish liquor resembling thin mucus, which mingles with the sperm at the time of its ejection, but for what particular purpose has not bcen satisfactorily determined. 
THE LESSE PROSTRATEs are placed at the sides of the urethra contiguously to the posterior part of the bulb, where they lie upon the branches of the ischium. Each of them is about equal in bulk to one of the lateral lobes of the large prostrate. They are included in loose cellular sheaths continuous with the substance of the triangular muscle. In figure they are flattened ovals. In color they are also a pale brown; but it is a much lighter shade than that of the large one. Their exterior is even and indicate their structure to be of the conglobate kind. The interior exhibits a more uniform appearance: a longitudinal section through the middle displays an arborescent membranous structure, which is seen by the use of the blowpipe to be the system of excretory ducts, whose papillary terminations in the urethra have been already pointed out. These glands also secrete a peculiar fluid which is ejected in commixture with the sperm. 


\section{LECTURE; LVIIT.}

\section{On the Female Organs of Generation.}

\section{T}

HE sexual apparatus in the female are usually classed for the convenience of description into those organs which are external and those which are internal: the former being constituted of certain parts that admit of examination without the aid of dissection; the latter of such as are so placed that they cannot be brought into view but by laying open the cavity of the pelvis. The external organs comprehend the vulva, labia, commissura, clitoris, and meatus urinarius.

THE VULVA or PUDENDUM is the broad slit commencing a little below the anus and extending downward between the quarters. A more correct term for it is the fissura magna or sinus pudoris, since the word vulva is not infrequently used to denote the external parts altogether, taken as a whole. This fissure is longest and most conspicuous in breeding mares, in consequence of some loss of tone sustained by the labia in parturition. The space between the anus and vulva is what we call the perineum in the female. The two lateral prominences forming the fissure are 
The labia PUDENDI. They are composed exteriorly of doublings of the common skin, which here exhibits a particularly fine and soft texture, is hairless and in most mares black, and is preserved moist and supple by a sebaceous secretion continually exuding from minute pores in their opposed surfaces. But the labia owe their bulk principally to adipose substance, and this accounts for the variations in regard to prominence they undergo in conformity with the age as well as the embonpoint of the animal. With this fatty substance is blended a fibrous texture, and so intricately that the one cannot be demonstrated separately from the other, nor can their mutual connection and arrangement be minutely made out: this fibrous texture gives the labia firmness, resistance, and elasticity. Between this mixed substance and the skin we find scattered fasciculi of pale fleshy fibres: these come from the perineum, grow larger and more conspicuous as they descend, and form a union below the inferior commissure with similar fasciculi coming from the opposite labium. Some consider these fasciculi collectively as forming a distinct muscle, and give them the name of sphincter vaginc. They will unquestionably perform such an office; but, I know of no occasion on which they appear to be so manifestly called into action as when a mare, just after staling, is ejecting the stagnating drops of urine from the vagina by repeated eversions of the labia and os vaginæ. The labia are lined by a mucous membrane (the same as that which lines the vagina) which is continuous with the skin covering them externally, has a smooth humid surface, a pinkish hue, (unless it be at the season of the vene- 
real ostrum, when it is deeply reddened,) and is perforated by the discharging mouths of numberless mucous follicles.

The Commissures are the parts uniting the labia above and below. The superior commissure is extended into a sharp angle and joins the perineum; the inferior is obtuse or rounded off, and is bounded by a hollow, the fossa navicularis of some, at the bottom of which is lodged the

Clitoris. This miniature resemblance of the male organ is brought into view by widely dilating the vulva; and not only in its appearance is it strikingly like the penis but it is found to bear the same close comparison in structure. Its extremity is invested in a doubling of thin and delicate skin which is hairless and commonly marbled, or, if not, black, or wholly white: this part corresponds to the prepuce of the penis. It is firmly attached to the pubes by two short ligamentous prolongations, forming its roots, which unite into a cylindrical body, from two to three inches in length in a state of distention, and this consists of two spongy canals separated by a pectinated fibrous partition and inclosed within a dense, firm, white tunic : in fact it is a structure altogether the counterpart of the corpora cavernosa penis. To this is added a bulbous end similar in form and composition to the glans penis ; and through it is a perforation out of which may be squeezed a sebaceous matter that serves to anoint the part and preserve its aptitude for that sensual enjoyment of which it (as well as the glans penis) is known to be peculiarly susceptible. To the clitoris belong a pair of muscles named singly the

Ereclor Clitoridis. They take their origin from the 
perineum, where their fibres are mingled with those of the sphincter ani, and are inserted into the upper and back part of the clitoris. Their office is to erect that body and protrude it into the vagina in the act of coition.

THE MEATUS URINARIUs may also be shown without the aid of dissection, and this is a stage of the inquiry in which we ought to make ourselves well acquainted with its situation; but, as it is united in structure with the vagina, I shall postpone its consideration until I have described that canal.

\section{The Internal Parts}

Are the vagina, uterus, Fallopiun tubes, fimbria, and ovaria.

THE VAgINA is a musculo-membranous canal of large dimensions extending from the vulva to the uterus.

It is situated within the cavity of the pelvis, having the bladder below and the rectum above it, to both of which it has broad cellular attachments in addition to the reciprocal connection of all three parts through reflections of peritoneum. To the rectum it is closely and firmly attached, by cellular membrane, along its upper side; to the cervix, and to the upper half of the body of the bladder, which is not covered by peritoneum, its inferior and anterior part is connected by a broader and looser cellular band; and the extreme parts of the canal in front, both superiorly and inferiorly, are applied against the peritoneal pouches formed between the uterus and rectum above, and the uterus and bladder below.

The figure of the vagina, when it is distended, is 
that of an oblony cylinder; but in the collapsed state its sides are in contact, and it will vary its form according to the full or empty condition of the bladder and rectum; and when they, especially the latter, are distended the long axis of the canal will be from side to side, with the exception of its orifice which will have the long vertical diameter preserved by its union with the vulva. The most capacious part of the canal is the posterior, there it even exceeds the dimensions either of the bladder or rectum; the part joined to the meatus urinarius is considerably contracted, but from thence it gradually widens to the outlet.

The length of the canal is about eighteen inches. Its course is horizontal : its axis however does not exactly preserve the straight line, it rather shows an inclination to the curve of the rectum.

The vagina at its commencement from the vulva is much thicker in its parietes than elsewhere, which is partly owing to a coating of strong muscular fibres, and partly to two cellular or cavernous membranous bodies, one upon either side, which have some resemblance in structure to the interior of the glans penis; they are inclosed in membranous covering's, surrounded by adipose substance, and show signs internally of having recently contained blood. I know of no appellation in use for these bodies: M. Grrard calls them "bulbes vaginals;" I see no impropriety in naming them the corpora cavernosa vagine.

The vagina in composition is partly muscular and partly membranous. The orifice of it is clothed in that strong, red, circular, fleshy band which forms the sphincter vaginæ, and the adjoining part of the canal is also encircled by some considerable fleshy fasciculi ;

PART III. 
but the part most compacted, and most thickly and regularly coated with muscular fibres, is that which is contracted-which receives the urine as it flows from the meatus urinarius, though the fasciculi hereabouts are not so red, nor so strong, as those blended with the sphincter. Farther forward than this the vagina is substantially composed of membrane; there are indeed colorless fibres taking various directions scattered over its surface, but they present no very unequivocal marks of muscularity though they are commonly considered to be of that nature: fibres apparently similar to these are also found underneath the red fasciculi posteriorly, but there they take a longitudinal course.

The membrane of the vagina, the part of which it is integrally constituted, is one of the mucous class, and one that possesses considerable density, extensibility, and resistance. Its exterior surface is rough and flocculent from the adhesion of muscle and other parts. Its interior is smooth and polished, humid from secretion, and has a very pale pinkish cast; unless the mare be under the influence of the venereal œstrum, and then its redness is considerably heightened and its secretion abundantly augmented. In the ordinary state this membrane is thrown into folds, larger in breeding mares than in others, technically called ruga, which proceed without much regularity from its outlet to its uterine end. Follicles furnishing the mucous secretion are scattered about underneath the membrane; and their orifices are generally most. conspicuous within the more capacious part of the canal.

Considerably in advance of the clitoris, altogether about four inches in the collapsed state from the entrance of the vulva, is an opening leading from the 
lower part of the canal large enough to admit with ease any one of the fingers: this is the orifice of the meatus urinarius, and it is a part with the situation of which we cannot be too familiar as upon the knowledge will principally depend our skill in passing the catheter. The conduit into which it leads is about two inches in length; and its course is downward and forward : it is surrounded below by the muscular fasciculi of the vagina, and covered above by its lining membrane. 'The instrument therefore (which should have more curve than is commonly given to it) must be introduced so as to follow this oblique direction, which will be accomplished by elevating the handle of it as soon as the point is pushed into the meatus. The orifice of the meatus is guarded by a doubling of the vaginal membrane, which hangs over it and serves the purpose of a valve : care must be taken to elevate this by means of the instrument; or, should there be any difficulty experienced in doing this, the left hand may be carried per vaginam to the part itself as an assistant.

The large and conspicuous cauliflower protuberance at the bottom of the vagina, is the cervix uteri : it will be described with the

UTERUS. The womb is a hollow, musculo-membranous organ, united to the anterior part of the vagina, and destined for the reception of the fruits of impregnation.

The uterus of the mare is one of a striking and peculiar figure; its body, which is the bulky, oblong part, bifurcates anteriorly into two cormua or horns: I hardly know what the whole is like in figure, unless the vagina be taken with it and then in a mare that has never been fecundated they very much resemble 
altogether an insect of the beetle tribe, the vagina representing the bottle or body, the uterus the head and neck, and the cornua the horns. But, during the period of gestation the womb undergoes an almost incredible augmentation in volume; and it never afterwards recovers its original or virgin state of contraction. We distinguish then in the uterus, body, horns, neck, and mouth.

The body is the oblong or cylindrical part, growing out of the anterior portion of the vagina, in the centre of which it is terminated internally by the os uteri, and is giving origin in front to the horns. Its upper and under surfaces are convex, most prominent towards the middle; its corners or angles are round; and it is evidently formed altogether to give capaciousness within. This part lies wholly within the pelvis, between the bladder and rectum, and is entirely covered by peritoneum.

The cornua or horns, having risen from the body at an acute angle with each other, take a progressively divergent course to the loins under the broad plates of the ilea; they are therefore not entirely confined to the pelvic bason. Their length and size will be much greater in breeding mares than in others, though their volume altogether will be less when compared with the capacity of the uterus: indeed in virgin mares the diameter of a single horn is little exceeded by that of the womb itself. In figure they are cylindrical; they bend upward in their course; and they terminate in round extremities, to which are loosely appended the ovaries, through the medium of the Fallopian tubes.

The cervix or neck of the uterus is the rugose portion protruded backward into the cavity of the vagina, 
which from its flower-like appearance the French have fantastically named "la fleur epanouie ;" it is a part however that can only be said to be demonstrable in a virgin uterus in the undistended state-inflate these parts and the cervix will disappear; and one that during gestation undergoes certain remarkable changes. The vertical slit or oval-shaped aperture in its middle is the os uteri or mouth of the uterus. The peculiar florulent aspect of the cervix is owing to its being enveloped in a prolongation from the lining membrane of the uterus, which is uniformly puckered into many rugk as it passes through the os uteri: around the sides of the cervix these rugæ become continuous with those of the vaginal membrane. This is a part that receives an especial abundant supply of mucous secretion.

Independently of its union with the vagina, the uterus is confined in its place by two broad productions of peritoneum which attach it to the sides of the pelvis, named the ligamenta lata uteri. They are formed in this manner-The peritoneum which is reflected from the bladder covers the under parts of the body and horns of the uterus, their upper parts receiving a similar covering from another portion of the same membrane derived from the rectum; the two portions then unite at the sides of the uterus and continue in union to the opposite parts of the pelvic parietes: this arrangement of the membrane in fact divides the pelvis into two cavities, or rather blind pouches, one existing between the uterus and bladder, the other between the uterus and rectum; at the same time it serves to connect these parts mutually to each other. The cervix consequently receives no peritoneal covering. These ligaments sus- 
tain between their layers the vessels and nerves belonging to the organ, preserve its equilibrium during gestation, and aid in retaining it in its proper centrical situation.

Underneath the peritoneal tunic of the uterus, united to it by a short cellular tissue, is a dense fibrous structure possessing a considerable degree of strength and extensibility, which is generally thought to be muscular; and the fibres have certainly that appearance, though their direction is very various, some running circularly, others deeper-seated longitudinally, and others again decussating these. But, about the cervix in particular, where the fibres are strongest, most collected, and often have a pale bloody tinge, the muscular structure is most assumed; though, after all, the presumptions of its muscularity scem rather to be drawn from a physiological than an anatomical source. During the period of gestation this tunic not only experiences considerable extension, but may be proved to undergo veritable accretion. Inwardly it is opposed and adherent to the internal tunic ; but their connection, which is also cellular, is looser than that existing between it and the peritoneal covering.

The cavity of the uterus, having the same irregular figure as the exterior, has three outlets:-one of considerable size communicating with the vagina, through the os uteri ; two others of very small size through the extremities of the cornua, leading into the Fallopian tubes, the situations of which are denoted by two little whitish eminences visible upon the internal surface. The membrane lining the cavity is one of the mucous description; it has a more vascular and villous appearance than that coating the vagina, but, like it, is thrown 
in the collapsed state of the organ into numerous rugæ, which pervade the cornua as well as the body and bear some comparison to an irregular network: these rugæ are larger and looser in mares who have bred several foals. The surface is everywhere copiously furnished with mncous secretion by subjacent follicles whose orifices are visible to the naked eye.

The Fallopian tubes are two trumpet-shaped or conical canals, having a remarkably serpentine course; munning within the folds of the ligamenta lata from the extremities of the cornua uteri to the ovaria.

The tube commences by an aperture in the cornu having an elevated whitish margin, which is scarcely large enough to admit a small silver probe; from this it proceeds forward, folded in peritoneum, and extremely convoluted until it reaches the ovary, to which it becomes attached; it then begins to enlarge in its diameter, grows less convoluted, and serpentines along the posterior side of the ovary, until it has got to the fissure of that body, at which part it ends in a fringed doubling of membrane, named the corpus fimbriatum, by a funnel-shaped irregular opening, which is turned backwards, large enough to admit an ordinary-sized black-lead pencil. The corpora fimbriata hang over the fissures of the ovaries, concealing them indeed from view, and are unattached and float about loosely with those bodies within the abdominal cavity.

These tubes, when stripped of their peritoneal ellvelopes, show a fibrous texture, and when opened exhibit a rugose, villous, mucous surface, very similar to that of the internal membrane of the uterus. They are amply supplied with blood-vessels which make their way to them between the layers of the broad ligaments. 
THE OVARIA or female testicles are two egg-shaped bodies, situated farther forward than the Fallopian tubes within the cavity of the abdomen, at the distance of an inch and a half from the cornua uteri. They receive close coverings from the anterior portions of the ligamenta lata, by processes of which they are loosely attached to the spine in their situation beneath the ilea and a little behind the kidneys: indeed the left ovary has a peritoneal attachment to the left kidney. So that if it were our intention to extract them, the incision should be carried along the crista of the ileum, and the hand introduced in a direction backward and inward.

These bodies are about the size of walnuts. They are not regularly oviform: they have deep fissures in their posterior sides which are occupied by the corpora fimbriata. Taking them and the Fallopian tubes together, they bear a striking resemblance at first view to the testicles and their ducts in the male.

Underneath their peritoneal coverings, the ovaries have whitish fibrous tunics, dense and inelastic in their texture, which warrant the comparison generally made of them to the albugineous tunics of the testicles. Internally the ovaries are composed of a whitish spongy substance, in which are in some instances found little vesicles containing a yellowish glairy fluid, in others one or more dark yellow or brownish substances named corpora lutea: the vesicles are the ova which from impregnation take on further development; the corpora lutea denote the parts from which vesicles have burst, and consequently only exist in the ovaries of those mares whose organs have been engaged in the generative process. Prior to the age of sexual intercourse 
these bodies are small and white; but as soon as the season of copulation is at hand, they grow large, redden externally, and present many yellow spots or streaks through their substance within.

The arteries supplying all these parts are-1. The vuginal artery, a branch from the internal iliac, which accompanies the obturator artery and then dips down upon the side of the vagina, upon which it branches out in various directions, sending some ramifications in its way to the rectum, and transmitting others to the uterus and bladder. 2. The uterine artery, a branch from the external iliac, coming off after the ilio-lumbar, and distributing its branches principally upon the cornu uteri. 3. The spermatic artery, coming from the aorta, is convoluted in its course, and supplies the ovarium, corpus fimbriatum, and Fallopian tube.

The nerves are derived from the hypogastric plexus. Both the vessels and nerves take their passage between the layers of the broad ligaments, insinuate themselves underneath the peritoneal covering, obliquely pierce the substance of the muscular tunic, and ramify extensively between it and the internal membrane.

Before I proceed to the physiology of generation I shall give the description of two glands, which, though unconnected with these organs in an anatomical point of view, are in function concurring to the same important end-the continuation of the species: these are the

\section{Mamma.}

THE mammæ, vulgarly called the udder, are two flattened oval-shaped bodies depending between the thighs from the posterior and inferior part of the belly.

In quadrupeds, with but few exceptions, this is the 
situation of the mammæ; in the human species and in the ape tribe they are attached to the breast. The advantages resulting to the quadruped, who has no power of erecting himself or handling his young, from this situation of the mammæ, are, that they are well protected by the trunk above and the thigh on either side from external injury, that they are conveniently placed for the foal, and that they do not in the least interfere with progression.

In fillies and virgin mares the udder is so small that there hardly appears to be any; and in mares who have not had more than one or two foals it also regains nearly its original flatness; but in this last instance the dugs are commonly left larger, looser, and more pendulous than formerly, in consequence of the reiterated extension of them by the foal: a circumstance on which we may rely with tolerable certainty for a knowledge of this fact. In mares who have had several foals the udder remains prominent or pendulous and has a flabby feel; and this is a state we cannot well mistake. Towards the latter end of gestation this part undergoes further evolution, swells and grows firm to the feel, and becomes distinctly visible as we stand behind the mare. Within a few days of parturition secretion begins and the udder grows turgid with milk; it does not however acquire its full distention until the foal has drawn it for a few days, from which time it maintains its volume with little variation during the period of lactation. Soon after the foal begins to forsake the teat the secretion of milk diminishes, and this is followed by a contraction of the bag which goes on gradually until it has resumed nearly or quite its former flatness. In a state of full evolution then the udder assumes a hemispherical 
form, and acquires a firm plump feel; at other times it is soft, flabby, more or less pendulous, and has no definite figure nor volume.

The outer covering of the udder is an extension from the common integuments of the belly; it is thin and fine in its texture, is commonly black, and is clothed with a few long downy hairs growing thinner as they approach the dug, immediately around which the skin is without hair. Underneath the skin, adhering to it by intervening cellular membrane, is spread over the gland a white, elastic, ligamentous covering, interspersed with several fibrous bands, which is derived from the faschia superficialis abdominis : this serves to give support and compactness to the glandular structure, and in consequence of detaching processes into the interior among the lobules to strengthen their interunion one with another.

The interior of the mamma has a light yellowish aspect, and evidently possesses a lobulated structure, which is held together by a fine cellular tissue here and there interspersed with granules of fat. It is constituted of glandular masses, irregular in magnitude and form and loosely connected one with another, each of which masses is composed of a number of lobules closely compacted and united together: a composition altogether that accounts for the loose or knotty feel which the gland is well known to possess. These insulated lobulous portions receive small arteries in numbers from the parent arterial trunk, from whose terminations arise (we do not precisely know in what manner) the tubuli lactiferi. These numberless little tubules, by repeatedly conjoining one with another, become at length several demonstrable canals, radiating from every part to as- 
semble in the teat, still uniting one with another in their course, and occasionally dilating into considerable reservoirs for holding the secretion. The lactiferous conduits are continued through the substance of the dug, wherein during its relaxation they are so serpentined or coiled that milk cannot spontaneously flow from them; but in the distended state of that body, or when it is drawn out in the act of suction or milking, these ducts are rendered straight, and the milk, either from internal or external pressure, is readily forced out.

THE PAPILLA or teat, which depends from the most prominent part of the udder, near its middle, is conical, black, and hairless. Its tegumental sheath is of the same description as that covering the bag, from which it is derived, only it is perforated at the apex by three holes to give issue to the milk; and underneath this is a second tunic which comes from the elastic ligamentous covering also of the gland. Through these openings, one of which is commonly conspicuous for its size, the milk is discharged from the conduits of the lactiferous tubes. When the udder becomes charged with milk it flows into the teat and distends it; and as the secretion is probably influenced in animals, as it is in the human subject, by anxiety for the young, the animal evinces this feeling by a state of distention of these parts. The dug, as has been remarked before, enlarges during the season of lactation, and it does not afterwards recover its original volume so nearly as the udder itself does, a fact, I repeat, that may lead, when the first sign is wanting, to the discovery of mares that have bred.

Sucking is apparently an operation purely mechanical. The teat is seized and so closely compressed by the lips 
of the foal that the imbibing effort which follows has a tendency to produce a vacuum in the lactiferous tubes now rendered straight from extension: this is counteracted by the pressure of the atmosphere upon the surface of the udder, and the consequence is, that the milk is forced from its reservoirs into the mouth in the same manner that water is impelled into the barrel of a syringe from raising the piston. 


\section{LECTURE LIX.}

\section{On the Physiology of the Organs of Generation, Male and Female.}

IN the course of our inquiries into the anatomy of these organs we discovered some remarkable points of correspondence between them in the female and in the male, to wit, the clitoris and penis, the ovaria and testes, and the os uteri and glans-penis; but now that we have come to their physiology we shall find that they are so mutually dependent for the exercise of their functions and conspire in such manner together to the common object of these functions, that they will conveniently and perhaps advantageously come into the same physiological investigation. This common object is no less than the devolopment and reproduction of new beings. It might be supposed that the part the male takes in fecundation is comparatively a very unimportant one; it must be remembered however, that the copulative act is the essential first cause, that therein the action of the organs is mutual and sympathetic, and that the result is the generation of a new animal bearing a likeness to one or both of the parents; from which it would appear, although the physical 
part of the male is simply to project the sperm into the female, who alone has the power of rendering it efficacious, that the influence of the sperm is much greater in the generative process than we seem to have any notion of, or at least than we have been able to reveal the nature of in physiology.

The office of the testes being to furnish the fecundating liquor (which we call semen or sperm) we consider them to be the most important part of the male apparatus. These organs do not descend into the scrotum for some time after birth, and it is not until the animal is considerably advanced in growth and strength that they carry on their glandular functions: this, the period of puberty, is denoted in animals as well as man by some remarkable changes both in body and propension. In man the pubes become covered with hair, the beard makes its appearance, the voice alters, the sexual organs undergo their full development, menstruation begins, and the mind is taken captive by a novel and irresistible influence. In quadrupeds this epoch is marked by luxuriant growth of hair, particularly of the mane and tail, by alteration in the voice, by the production of the crest or horns or both, by the emanation of a peculiar odor from the body, by the further evolution of the organs, and by the manifestation of the cestrum in the female and of an indomitable lustful passion in the male. All that we know about the secretion of the sperm, is, that it is elaborated in and by those capillary ramifications of the spermatic artery that have some indefinable communication with the tubuli seminiferi, and that these tubes are always to be found full of secretion; of which fact, in addition to the anatomical evidence formerly given, we have this 


\section{Un the Physiology of the Generative Organs.}

proof, that ligature of the spermatic arteries, though it does not mortify the testicles, destroys the prolific powers of the animal. In regard to the semen itself, it is a white viscous fluid, having a peculiar faint odor, and has been found to contain myriads of animalcula or vermiculi, as they have been also denominated from their supposed resemblance to worms; not only however have conjectures been broached about the form and size of these almost inconceivably minute corpuscules of vitality, but chimeras have found their way into print even of their sex and mode of generation. To chemical analysis, according to GIRARD, the horse's sperm yields four-fifths of a peculiar animal matter; the remaining ingredients being mucus, muriate of potash and soda, and carbonate and phosphate of lime.

The removal of the testicles not only deprives the animal of the power of impregnation but even renders him after a time insusceptible of any sexual appetite, and is attended with other enervating consequences. Sir A. Cooper has informed us, that a man from whom he took both testicles continued to feel venereal desires for some months afterwards, and to experience the usual emission and sensations in the performance of the coital act, but that both the power and inclination gradually grew weaker during the time, and that he became so perfectly emasculated at the expiration of a twelvemonth that he was not even capable of an erection. In like manner castration operates in horses, not only depriving stallions of their amorous fury but converting the most outrageous into meek and even spiritless geldings: in addition to which we have to notice this curious fact, that, should the operation be performed at the time that the animal is changing or about 
to change his coat, the process will be interrupted or suspended altogether until the return of the shedding season, and in the instance of the deer the horns will not be cast as usual during that year. But the loss of one testicle does not take away or even diminish (that we have any proof of ) either the procreative faculty or the sexual appetite: rigs appear to get foals as well and to cover as frequently as stallions, nor do we perceive any difference in men under such circumstances; we may therefore conclude that the popular notion of their comparative imbecility is not well founded. There is this remarkable difference however in the generative functions of animals in general and those of the human species, that the former only show themselves in vigour at certain seasons whereas the latter are in a continual state of activity; and this seems to go far to account for the comparative moderation and governable nature of the amorous passion in the latter: this periodical excitement has such an influence on the testicles in birds that they grow large in the coupling season, but shrink into very small size when they are no longer required to furnish secretion.

The use of the vesiculæ seminales has long afforded subject for disputation, and even at the present day admits of more than one explanation. The ancients conceived that they generated the semen. This erroneous notion was no sooner discarded than followed by the plausible one, that, although they did not form, they contained semen, and were to be considered as reservoirs for that fluid. Mr. HUNTER was the first to erect a doubt in opposition to this theory; and he ultimately adduced some ingenious experiments toge- 
ther with several well-selected cases to show that this opinion was not founded upon fact. Among other observations made by this distinguished physiologist, he discovered that these bodies contained to all appearances and tests the same fluid in geldings as in stallions; and that in men who had for a long time before death been deprived of one testicle there was no discoverable difference whatever in the properties of the fluids found in the rightand left vesiculæ. In fine, if $\mathrm{Mr}$. HuNTER's researches into their physiology be not of that character that would warrant us in coming to the same conclusion with him; at least they have led to a demonstration that the vesiculæ do themselves furnish a particular fluid of the nature or appearance of mucus, and that the semen, if it really does ebb from the direct channel into these bodies, is therein so mixed with their secretion that it evades the ordinary tests of detection; and further, that in this diffused or diluted state it is ejaculated from the penis. This doctrine-that no semen enters the vesiculæ, requires some satisfactory explanation of the production of seminal emissions in the very short space of time in which they are known to be demanded and furnished. Mr. HunTER supposed that the testicles were at the moment excited to action sympathetically with the passions of the mind; and we may add that the secretion which it is known they always contain probably requires but little addition to render it available: the extreme length, exility, and tortuosity of the seminal tubes however appear to weaken much the force of this supposition; while, on the other hand, the opinion that semen is collecting at other times within the vesiculæ 
receives no small weight from the experimental result, that fluids injected into the vasa deferentia find their way into these sacs.

The sperm receives further augmentation at the time of its emission from the prostrates. The secretion furnished by these glands is white, opaque, thick, and viscous, bearing considerable resemblance to mucus, and appears to be well adapted as a vehicle for the semen; the ejaculation of which it is believed to facilitate by the addition made to the bulk of the latter, and by the glibness it diffuses over the seminal fluid as it passes along the urethra. So far as a menstruum or vehicle is operative, it may be said also to contribute to the efficacy of the semen, injected, diffused, and mingled with other secretions as that fluid must be in the organs destined for its reception in the female.

The accomplishment of sexual congress requires that the penis be in a state of erection: the testicles at the time are drawn up close to the belly by the cremasteres, and the contractile powers of the vesiculæ and vasa deferentia, aided by the accelerator urinæ and levatores ani, are supposed to be exerted in the ejaculation of the seminal fluids. The distention of the penis with blood, which is the cause of erection, is occasioned by an increased action of the pudic arteries and the consequent accelerated influx of blood into the corpora cavernosa and thence into the glans penis, in which accelerated local action the veins do not participate from the interposition between them and their arteries of this spongy and vascular structure; the result therefore is congestion of blood within the penis, and this effect is considerably promoted as the distention increases by the mechanical obstruction to 


\section{On the Physiology of the Generative Organs.}

the return of blood occasioned at its root by the approximation of the organ to the pubes and the compression of the trunks of the veins there between it and the bone.

In the rutting or covering season animals are found to grow restless and unruly, and to be disposed to ramble from their pastures; indeed, unless permitted to seek their mates they will often refuse their food, and even undergo considerable privations, suffering extenuation of body partly from this cause and partly from nervous disquietude: so domineering and influential in the system is the periodical sexual passion. Numerous and remarkable are the instances in proof of this ardent amorous attachment among quadrupeds : the train of followers allured by a bitch at heat, the number of miles a dog-fox is known to ramble in quest of the female, and the implacable fury of the bull, are among the more familiar of them. No sooner does the stallion come to the knowledge of the horsy condition of the mare than he becomes fired with an energy and a courage unknown to him at other times; nothing will pacify him so long as he imagines she is within his reach, he grows impetuous and unmanageable and requires means of restraint unused before to confine him in his stable or prevent him from breaking loose whenever he is led out. At the time that he is suffered to approach the female his whole frame exhibits the height of animation: his nostrils are dilated to their utmost to gratify his lecherous sniffs; his eyes flash with nervous and passionate expression; his actions and carriage altogether, while they display gracefulness, manifest intense vigor; and he evinces no less irascibility towards any one who would thwart him in his 
impetuous course than lustful ardor for the object before him. The presence of this influence in the system is attended in some animals with a peculiar fetor of from the body, and a remarkable hardness and toughness of muscle which renders them unfit for food: the hegoat is a striking instance of these changes; but they are also manifested by the stag, ram, bull, and others. Absorption of accumulated semen has been offered by way of assigning a cause for these phenomena; and, granting the fact-one that the constant state of plenitude of the seminiferous tubes and the presence of numerous absorbents in the gland render highly probable, the rationale, as far as it goes, is very plausible, supported as we find it to be by the link in this chain of observations, that castration removes or greatly mitigates these effects.

By implanting such a vigorous and irresistible impulse into the sensual constitution of animals has $\mathrm{Na}$ ture excited the congress of the sexes and insured the perpetuation of their species; and so salutary is the moderate or seasonable performance of this act that animals are found to be improved both in health and strength by it: but the line of moderation is not to be stepped over with impunity; preternatural excitement of the organs-overstraining their functions, never fails to shorten the term of their capabilities to renew their offices, and, if carried to excess, to prove highly injurious and destructive to the system altogether. It is well known that bulls who have much gregarious intercourse seldom retain their lustful vigor longer than two or three years; and that stallions also who cover many mares in the course of the season suffer by it a. permancnt reduction or debility of stamina. 


\section{On the Physiology of the Generative Organs.}

Let us now pass over to review the functions of the organs belonging to the female. I have already made some allusions to what is called the covering or rutting season, by which is meant that period of the year during which the female parts are in a state of aptitude for giving due effect to the sperm conveyed into them by the penis of the male. This periodical condition of the organs furnishes us with a mark of distinction, as it relates to generation, between the human female and animals in general. Woman becomes at the age of puberty, and continues to be until the decline of life, the subject of a discharge from these parts which from its regular return with the moon or month has got the name of catamenia or menses; this flux has been found to consist of blood destitute of fibrine, and is supposed not to be a mere sanguineous effusion but to result from a sort of secretory process carried on by the mucous membrane of the uterus. The natural effect of this discharge is, to preserve the organs in a perpetual fit state for conception; for it is found that women who do not menstruate from the uterus do not conceive. Animals are not so by nature. Though there are some appearances, vulgarly denoted by the term heat or heats, that might lead us to an opposite conclusion, such as determination of blood to the parts, more or less mucous discharge, and that occasionally even tinged with blood, yet this is but an uncertain and infrequent return, much influenced by the season, by nomeans assuming that regular and determinate character of the menses in women, and is one that only continues during the influence of the sexual cestrum, and so long, but no longer, sustains the organs in a fecundating condition. Mares become the subjects of this salacious influence at the approach of 
On the Physiology of the Gienerutive ()rgans. 10:3

summer, when they are said to be horsing, and show it by redness and prominency of the vulva, and by the emission at intervals in considerable quantities of a whitish mucous fluid, the odor of which proves a peculiarly potent incentive to the male : these appearances are found to be accompanied with general turgescence, reddening, and increased excitability of the whole genital apparatus, and more particularly of the uterus which now opens its mouth, dilates, and disposes its cavity for the reception of the prolific fluid. Some mares will take the horse shortly after they have foaled; but the ninth or tenth day is the regular period of return of the œstrum, which ordinarily continues for four, five, or six days; after which it commonly returns at intervals of three weeks. Anong the circumstances, natural and artificial, that have any influence on the production or return of the œstrum, we may mention condition, temperature, and various artificial stimulants as the principal. Animals who are in the vigour of health, without showing much disposition to obesity, who live well, are not over-worked, and particularly such as are fed upon stimulating provender, are more so disposed than others: indeed mares low fed, ill treated, and hard worked, seldom show that they are horsing at all. Animals in general, particularly birds, copulate during the spring or summer; to which fact may be added another, though less notorious not less true, to demonstrate the operation of heat, which is, that animals transported from warm to cold climates seldom copulate and more rarely breed after their removal: mares are commonly found horsing in the month of May or June. There can be no doubt but that there are various substances promotive of this condition of the parts, and their effect appears to be simply that of excitation; certain of these will 


\section{On the Physiology of the Generative Organs.}

operate through the system, the others are mere local stimuli: some grooms, I am told, are in the habit of very roughly using such substances and occasionally inflicting thereby considerable local injury. Animals are also capable, other circumstances favoring, of having certain salacious sensations aroused in them by the male which proves a great promoter of the œstral condition : this is well known to breeders, who are in the habit at the proper season of having mares they are desirous to have fecundated frequently teased by the stallion. Age must also be taken into this account: mares are incapable of fecundation prior to the twentieth month or second year of their age, and become barren in advanced life.

The horse no sooner quits the mare after copulation than she rejects, it would appear, the greater part if not the whole of the prolific liquor she has just received; to prevent which it is a common practice with grooms to press the tail or a wisp of straw forcibly against the vulva so as to close the orifice. There is also a practice in vogue of dashing a pailful of cold water upon the loins or quarters, with a view of diverting the excitability of the genital parts. The female ass is often well beaten or spurred afterwards as a still more effectual diverter. GirARD tells us, that the Arabians fatigue the mare they intend to have covered in order that she may repose after coitus and so lessen the uncertainty of being fecundated.

Impregnation ordinarily puts an end to the œstral discharges by causing permanent closure of the os uteri; this however is not invariably the case, for some mares will receive leaps and appear to be horsing even at an advanced period of fecundation. It becomes a very interesting question in opening the abstruse subject 
On the Physiology of the Generative Organs. 105

of the physiology of female generation, to ask to what part in particular the semen is designed to be applied; for, since this fluid is no less than the primum mobile of generative action it cannot be an affair of indifference in what manner it becomes disposed of : it must either be deposited in the vagina and there remain; or be injected into the uterus and lodge there; or pass on through the Fallopian tubes to the ovaries. However simple this question may appear, hardly any physiological problem has given birth to more controversy. HALLER says, that he once found semen in the uterus of a sheep. Mr. HunTER discovered it there in a bitch that was killed instantly after copulation. Dr. HАIGHToN has made a series of experiments with rabbits to throw light upon this subject which have led to some curious and interesting results. He divided the cornua uteri, the natural consequence of which proved to be, complete obliteration of them : this not only destroyed the power of conception, but all inclination to employ the means. After this, he divided one tube only, which in many instances was followed by conception : corpora lutea, which, according to the Doctor, are never formed but after conception, were produced on both sides, but foetuses only on the sound side. But how came it that conception took place when the communication between the ovary and uterus was intercepted? The experiment goes to show, that it is not necessary that semen should be brought into actual contact with the ovary to produce conception, since impregnation was not permitted until forty-eight hours had elapsed from the time the tube was divided. But why did not fotuses also form on the intercepted side since it is presumed that conception actually took place there? In 


\section{On the Physiology of the Generative Organs.}

answer to this the Doctor gives us the experimental fact, that the division of the tube at any period after conception but before the germs have passed into the fimbrix, will equally prevent the appearance of foetuses ; but, if the rudiments of the new animal have already passed into the tube, the actions of the parts suffer no interruption. To what, then, are we to ascribe conception? The Doctor answers, to sympathy. The subject admits, I think, also of a glimmer of light from a review of the genital parts in horses especially : the comparative lengths of the vagina and penis, and the adaptation in form of the end of the latter to the os uteri, afford presumptive evidence at least of the design of Nature, that these parts should come together.

The injection of semen then into the uterus appears to be all that the female receives from the accession of the male: the notion that she herself emits any seminal liquor in the act is now dropped; it being pretty well ascertained that the said emission is no more than a mucous discharge from the vagina. A knowledge of this fact might lead one to inquire whether the office of the male could not be supplied by artificial means, supposing that we could obtain in every case the proper masculine liquor. SPALLANZAN I has proved that this is practicable: he tells us that "he has succeeded as well as if the male himself had performed his proper function;" and a singular corroborative case is given by Mr. Hunter; but my limits are too confined to do justice to these interesting facts in detail, and therefore I shall pass them over.

We have next to investigate the phenomena and appearances following copulation and conception. On these points Dr. HAIGHTON's accurate and connected 
observations * particularly merit our notice. In rabbits (with which his experiments were made) the only observable difference from the first to the ninth hour after coition, the animal being in high venereal condition, was a greater turgescence of vessels of the parts, as if preparatory to some important action. 2. In twelve hours after coition projecting vesicles were seen that had lost their transparency and become opaque and red; from which, when punctured, a fluid of the same color escaped. 3. At 24 hours the vesicles projected more evidently, and their thickened parietes, manifesting the formation of corpora lutea, had become more apparent. 4. At 48 hours the vesicles seemed to be in the very act of bursting, and a semitransparent substance of a mucous-like consistence was beginning to protrude from some of them. The fimbriæ were preparing to receive their contents, for they had embraced the ovaria to such a degree that only a small portion of the latter could be seen. 5. In five days and twelve hours the foetal rudiments had escaped; but the cavities of the ovarian vesicles had suffered but little diminution. Their ruptured orifices were manifest. 6. During the third, fourth, and fifth days there remain ed but little vestige of cavity, consequently the corpora lutea may be considered as perfectly formed.

We may consider then, as received facts, that the germs or rudiments of the future beings exist in the ovaria prior to impregnation; that they escape at a certain time subsequent to impregnation from the ovaria into the Fallopian tubes; and that they gradually pass backward into the uterus. No sooner has the œstrum ceased and fecundation is insured than the

"Vol. Ixxxvii. "Plilosophical Transactions." 


\section{$108 O_{18}$ the Physiology of the Generative Organs.}

turgidity and reddening of the ovaria (in common with the other parts) subside, and one of these bodies takes on a new action. A little, black, circumscribed tumor makes its appearance in this ovarium, which is the calyx or cup wherein is formed or at least contained the ovum or germ; after the expulsion of the ovum the calyx exposes a black cavity which gradually closes in consequence of a thickening and growth of its parietes and then includes a vesicle containing a yellowish fluid, and thus becomes what is called a corpus luteum: a certain mark of impregnation whether there be any discoverable embryo or not. It is a curious fact that the fimbriæ should spread out, advance, and grasp the ovarium at the period of the bursting of the calyx and that they both should be so consentaneous in their actions. Not however that this law is universally unerring ; for extra-uterine fœetuses are heard of, and these are believed to have fallen into the belly from defective co-operation, owing to some mysterious cause or other, in these parts; indeed, for aught we know this is a lapsus that may happen much oftener than is made sensible to us, and the fallen ovum come to nothing. Unless the ovaria exist then in a sound or efficient condition the female not only loses all amorous desires but no longer possesses the faculty of procreation : even the removal of one ovary was found by Mr. HuNTER to have a marked effect on the fecundity of the animal. Spaying therefore is an operation that reduces the female to much the same state to what castration does the male.

It has become a question of some magnitude among accoucheurs whether it be possible to impregnate a female a second time in whom fecundation has already 
On the Physiology of the Generative Organs. 109

taken place. In consequence of the season of amour being limited in animals it is not an inquiry that we can pursue to much advantage ; but instances have presented themselves in human medicine in which foetuses of different degrees of development have been discovered in the same female, and such are usually adduced as instances of super-foetation: these cases however have not been received as conclusive evidence, and so the point still remains uncleared up.

The primitive source of the new animal and the mode of its production are subjects naturally creative of more than common interest in the mind, and have proved to be the fruitful beds of speculations and hypotheses. Such of them however as are worthy of our attention we may include in the three following propositions. 1. That the fœetuses exist ready-formed in the sperm of - the male, and only seek a nidus for their evolution and growth within the body of the female. 2. That the germs pre-exist in the female, and only require the stimulus of the masculine liquor to dislodge and develop themselves. 3. That the production is a neutral or rather a mutual one, both sexes concurring in its formation.

After we have been engaged in examining the various parts of the complex apparatus destined for the purposes of generation in the female it is rather extravagant or at least unreasonable to suppose that these several organs should have been formed merely to afford a habitation or a nidus to a being already created and only requiring further evolution and growth; indeed, knowing as we do that the embryo arrives in a very indistinct state of existence and at a very early period or being within the uterus, were this notion well founded 


\section{On the Physiology of the Generative Organs.}

I do not see what functions there would be left for the ovaria and Fallopian tubes to perform at all! This is an hypothesis therefore that has deservedly met with but few supporters. The opinion that germs pre-exist in the ovaries and only require impregnation to develop themselves, the ostensible father of which is the indefatigable SPALLAN Zan I, who first discovered pre-existent germs in a species of frog, has met with advocates among the most celebrated physiologists; though there are some formidable difficulties in the way even here. The semen being proved to be indispensably necessary to impregnate the germ or ovum, where or how does this operation happen? Dr. Haighton has shown that the marks of conception, corpora lutea, may appear though all communication between the uterus and ovary is cut off; but that foetuses will not be produced unless the ova have been received by the tube. This would appear to show that the ova are rendered prolific by the semen within the uterus; but, then, how comes it that foetuses are now and then brought to perfection within the Fallopian tubes and even within the cavity of the belly? since they can not receive any such seminal contact? It has been ascertained, if a bitch (say a greyhound) be lined by several dogs of various breeds that she will not bring forth more whelps than if her intercourse had been confined to one, but that she will probably produce one of each race: the explanation given to which is, that the ova arriving in succession from the ovaria in the uterus, fall into the seminal fluids of the different dogs. Again, in birds we know that a single coition is sufficient to fecundate a great number of eggs though they are produced at different and even remote periods. That the foetus then 
On the Physiology of the Generalive Organs. 111

comes from the female in the beginning there appears but little reason to doubt, especially after we have perused the mass of evidence brought in support of this opinion by SPALLANZANi both from the animal and vegetable kingdoms; but, still, the subject is open to have shadows of obscurity cast over it by some few facts no less embarrassing in their nature than they are notorious for their truth, among which may be mentioned - the likeness the offspring bears to its parents, both male and female; the transmission of ingenerate diseases, idiosyncracies, and discriminative conformations: and, to perplex us the more, it is observed that these peculiarities do not commonly transcend to the succeeding generation.

Then again, there is the hybridous production to add to this chain of mysterious facts. We well know that certain different species of animals will breed together and that the progeny will partake not only of the physical characters but of the dispositions of both; and the next wonder is, that this hybrid animal is not itself generate: such is the mule commonly so called. There are also hare-rabbits, wolf-dogs, fox-dogs, \&c. but the most curious productions of all are the jumarts, the offspring of the bull and mare, the ass and cow, and the bull and she-ass, an account of which has been rendered by Bourgelat. Some hybrid animals too have been known to propagate their kind; and by fecundating such for several generations from the seed of the male of the same species, animals have been produced bearing no resemblance whatever to the race from which they have originally sprung.

Lastly, I have to make mention of an occurrence which is calculated to throw a fresh light altogether upon 


\section{On the Physiology of the Generative Organs.}

this department of physiology. It has long been regarded as a physiological axiom, that impregnation is essentially necessary to the production of new beings, and we have already seen that it is the most prevalent opinion at the present day that such productions originate with the female. Refraining however from any comment on this doctrine I shall give the particulars of the occurrence alluded to. It has always been customary with my father (who has now served thirty years as Veterinary Surgeon in the Artillery) to inspect the abdominal and thoracic cavities in particular of horses destroyed for glanders. In one of these inspections a tumor, about twice the size of the testicle itself and of an oblong ovoid figure, was found four inches behind the left kidney, suspended by a slender fibrous band six inches in length. The tumor proved on section to be composed of a white, dense, fibrous capsule, approaching in its texture to cartilage, which in some places exhibited patches of the true osseous character. Its cavity is divided by septa crossing each other, of the same fibrous and patchy-osseous composition as the capsule itself, into nine unequal and irregular but perfectly distinct cysts. One of these cysts contains an ounce or more of common yellow fat; two others are filled with substances bearing strong marks of resemblance to cerebrum and cerebellum; in two more are contained two fangless molar teeth, displaying in perfection both bony part and enamel, and surrounded by unconverted pulpy matter; the sixth cyst shows an incisor nearly perfect; the seventh lodges a bony structure, an imperfect representation of the os maxillare superius together with its sinus and alveoli; the eighth contains medullary matter, and gives growth to a small cuspidatus or 
On the Playsiology of the Generutive Organs. 113

Lusk; the ninth encloses numerous long light-brownish hairs whose roots are implanted into globules of adipose substance. Two small arteries were discovered within the substance of the connecting band going to the tumor; but these unfortumately were not traced to their origin. The horse from whom this tumor was laken was to extemal appearance a gelding, was seven years old, and otherwise presented nothing remarkible.

Many years ago Dr. Barlue published in the Philosophical Transtictions the case of a girl who was not apparently more than $1: 2$ or 13 years of acge, in whom the hymen was perfect and the uterus undeveloped as it usually is at puberty, the other marks of which state were also wanting, whose ovarium was converted into fat, hair, and teeth: this girl, he presumed, could not have received impregnation, and the case led the Doctnr to depart from the received axion in physiology and offer a conjecture, that impregnation was not necessary to sich productions. A proposition that afterwards received corroboration from the discovery of a tumor containing hair and teeth in RuYcu's collection, which the Doctor informs us, is said by the celebrated collector to have been found in a man's stomach. The veterinary case howerch ahove detailed leaves no doubt of the fact, or in the Doctor's own words (for he has also published it) "fully extablishes the truth of my opinion." I do not mean to aver that this tmmor might not have been a degenerated or converted testicle; but whether it was or was not, the production is no less conclusive evidence in this point of view, and must be regarded as a phenomenon both singular in its kind and inexplicable in its origin. 


\section{LEC'JUE LX .}

\section{On Castration.}

THE lustful propensities of the stonehorse and his intractability under such influence have long rendered the practice of castration general in this country, where horses are in the habit of being stabled together and where the use made of them is such that they have frequent intercourse with one another. The stonehorse is in many respects an animal of exalted condition when compared with the gelding: his noble crest, the richness of his coat, his general gracefulness of form, his majestic and animated action, his tuneful and deep-sounding neigh, his hardihood, his vigor and incurbable mettle at the sight of the object of his appetence, all proclaim him to be in courage, constitution, and stamina, a superior animal to the comparatively mild, docile, and tractable gelding. Superiorities of a similar description are evinced by the bull over the ox, the ram over the wether, the boar over the barrow; but these entire males manifest in the season of concupiscence a formidable ferocity and intolerance of approach that we are not accustomed to see in the stonehouse, and which oftentimes render the manage- 
ment of them rather a perilous undertaking. But there are animals in which certain of these qualifications seem to be reversed, among which the geldings appear to enjoy the advantages of greater corporeal development; for after emasculation they are found to grow to an enormous size, to far surpass both in stature and bulk the uncastrated males: of this nature are the rabbit and capon in particular. Even these animals however are inferior to the perfect males in energy and spirit, and turn out to be comparatively meek and hamless creatures. Before attempting to unriddle these facts I shall bring forward one other which will go some way to reconcile their apparently contradictory results; and that is, that animals who are castrated prior to the age of puberty grow to a large size, but that after secretion has begun in the gland such effects are considerably less marked. Now, two explanations are offered in solution of these phenomena. It is said, that the influence this secretion is seen to have on the nervous system, and the vital energy expended in its production, will sufficiently account for the unequal growth and development of the body. There is no proof however of this consumption of material in the seminal secretion, and though it were allowed to be true, still, as the secretion at the early period of life is not commonly expended but, we believe, absorbed, no loss would thereby be sustained: indeed, there seem to be some reasons for believing that these effects are ascribable to the absorption of semen into the system, as I hinted before in speaking of the physiology of generation.

The common practice with breeders in this country is to have their colts cut as soon as the stones can be 
felt at the bottom of the purse, which can generally be done about the expiration of the first year; though there are veterinary writers who inform us, that castration is sometimes performed at three months: I should apprehend that the operation must prove an arduous one, and a perilous one too, because the testicles at this early period are, as we full well know, within the cavity of the abdomen. Occasional exceptions are made by breeders to this rule of cutting early, where particular individuals are defective about the crest and are of sufficient value to pay for this superadded chance of improvement. The hope is certainly not ill-founded; but it is too much, as is too commonly the case, to expect its fulfilment in the course of the following year; for, if the characteristic features of the stonehorse are referable to the absorption of semen, how can we reasonably look for their development before there is any sperm secreted?

The operation of castration in itself is one the successful performance of which gains us but little credit though its unfortunate issue may irretrievably lose a man his professional reputation. Why the scales should be so unequally and unfairly balanced is a question I will leave to those persons to decide who mete out praise and censure with characteristic arbitrariness and inconsideration, and who would heartlessly blast the professional name of a man because a favorite colt happened to sink under his knife, though that knife were handled never so dexterously, as if they themselves were all publicans in the temple and unsearchable and infallible in their undertakings! No man of liberal ideas and sentiments would think of attaching blame to a medical practitioner unless he had good 
reasons for believing that professional ignorance or inattention had occasioned the grievance; and no practitioner who is thoroughly master of his art and is conscious of having done his duty, has any reason for listening one moment to the reproach of his employer when that reproach is given vent to without either reason or reflection. What has not a little augmented this evil, is, that there are men existing in diminished numbers denominated gelders, who will take upon themselves the accomplishment of the operation at a small premium, and with whom the attached responsibility is an affair of little or. no consideration, since they are callous to all conscientious feelings, and enjoy a celebrity of too itinerant a nature to be injured by any local misdemeanor. Some of these gelders certainly operate with considerable adroitness; but this need not cxcite our surprise when we hear of the great practice they have, a practice exclusively confined to castration, not only of horses but of domestic cattle in general; watch them however when any thing happens contrary to the ordinary course of events, either during the operation or subsequent to it, and they will be seen confounded, at a loss what step to take, or flying at random to expedients and rapidly sacrificing at the shrine of ignorance a life that would have been perfectly safe had it been intrusted to the hands of onc acquainted with the rationale of his proceedings.

The object we have in view in the emasculation of horses is not to deprive the animal of the procreative faculty so much as to render him insusceptible of sexual excitement; though to accomplish the one we must naturally effect the other. Two operations are in practice for this purpose. One consists in so disorganizing 
the secretory structure of the testis that no semen can be ever afterwards secreted; the other in extirpating the gland: it has indeed been proposed to intercept the supply of blood by tying the spermatic arteries, but I have understood that this will not incapacitate the animal from experiencing amorous desires. A writer on castration in the human subject says, "the effect on the physical constitution of man is the same, whether the testicles be injured by contusion so as to break down their natural texture; whether the spermatic vessels be obliterated, leaving the testicles entire; or whether these organs be wholly renoved by excision."

Before any operation be unclertaken, particularly on horses who have passed the adult period, and more especially on such as have hunted or raced, it is a proper precaution to examine the scrotum in the erect posture in order to ascertain that it be free from hernia; for its presence is by no means an uncommon occurrence, at the same time it is one that ought to make us pause before we come to a determination to operate, if not deter us altogether from the operation. In several such cases the intestine has presented itself on the vaginal cavity being laid open, and this has been followed by such volumes of gut as to baffle all efforts to restrain or return them; for, soon after the escape of intestines, they become distended with air, red and turgid with blood from constriction at the ring, and these consequences would end in mortification did not humanity prompt the destruction of the animal before his sufferings experienced such dreadful aggravation. When manipulation proves unavailing in such a case, aided by the liberation of the contained air by puncturing the bowel with a pin, should that be deemed 
advisable, Professor Coleman proposes to make an opening into the belly, near the umbilicus, large enough to admit the hand, and through its introduction to draw in the protruded guts; were $I$ in such an unfortunate dilemma however, I think I should perfer dilating the abdominal ring, the scrotal opening too if necessary, proceeding in fact as I would in operating for hernia. Should we succeed in replacing the bowels we are to give up all thoughts of extracting the testicle; for, when we have strongly united the lips of the external wound by the quill suture we shall find that the testicle will materially assist in blocking up the passage and preventing the descent of the bowel.

The season of the year, should the operator not be confined to any particular time, is also to be taken into consideration. I would not operate during the season of changing the coat, nor even just prior to that period, for fear of interrupting the process or checking it altogether; neither would I choose frosty nor sultry weather; but above all it is advisable to suspend the operation when the files abound. If the subject have passed the colt period of age I would recommend a dose of aperient medicine before the operation be undertaken, unless he should be already living on green food and then this preparative is unnecessary. He may also be kept fasting the night before.

THE OPERATION, as I stated before, has for its rationale the incapacitation of amorous excitability, and this object may be carried into execution, by effecting a permanent cessation of the secretory functions of the testicle or by the entire extirpation of the gland. The practice in some parts of the Continent is to place the testicle between two hard substances and bruise it in such 
manner and degree as to destroy its texture; at least, this is the object of the bruiser, though the veritable cffect is to excite inflammation which commonly ends in the effusion of adhesive matter and ultimately converts the glandular structure into a shrinking mass of callus or schirrus. I should be under apprehensions myself however of the supervention in some of these cases of a suppurative or even a gangrenous termination, and of an accompanying peritonitis or febrile commotion of system that might end fatally.

I have heard of excising the epididymes without the testicles, and portions of the vasa deferentia with them. There can be no doubt but that the operation would be followed by a loss of the faculty of procreation; but the question to us is, will it reduce the animal to a state of imbecility or placability? because, unless it have such an effect it will not answer our purpose.

Various modes have been put into practice of removing the testicles. The simplest, and I may add the barbarous one, is that of tearing them out. The stone is first laid bare by incision, then firmly grasped by the hand and torn from the bag. In young animals this operation is not found to be followed by any hemorrhage of consequence: the cord is the part that riptures, and the licerated arteries retract within its fringed ends and become plugged with coagulum until they are sealed in the ordinary way by effusion. Lambs, pigs, rabbits, fowls, and other young or small animals, and I understand in some parts of the country yearling colts, are thus castrated. Professor CoLeman has seen this operation (which may be literally called extraction) tried on an old stallion with success: one testicle was torn out without any subsequent alarming hemorrhage; 
and after the lapse of a few days the other was extracted: had both cords been ruptured in the first instance, for obvious reasons fatal hemorrhages are likely to have ensued.

Excision has been practised : the same operation that is performed on the human subject. An incision is extended from the abdominal ring nearly to the bottom of the scrotum, in a line with the cord, which is thereby laid bare. The cord is then freed from its surrounding comnections, and a ligature carried through it between the vas deferens and the blood-vessels so as to include only the latter, and tied with sufficient force to stop the circulation. Next, the cord is divided half-an-inch below the ligature; and lastly, the testicle is dissected out of its cellular bed. The lips of the external wounds are brought together by suture, and the ligatures upon the cords are suffered to hang out between the stitches. The chief objection to this mode of castration in horses is their disposition under such circumstances to peritonitis, which arises from the abdominal ring remaining open: inflammation is readily propagated along the cord, from the scrotum into the abdomen, and it appeirs more likely to follow the application of ligature than that either of the potential or actual cautery.

Before I enter on the consideration of the mode of extirpation generally adopted in this country I shall give some instructions for managing, casting, and fettering the subject for the operation. Let us take by way of example a colt that not only remains up to this time unbroke but one that has not hitherto been haltered. The first thing to be accomplished is to contrive to slip upon his head a flat hempen halter, with which it is advisable, should he prove very refractory, to tie him up for a time that he maty have an opportunity of abatine his 
repugnant spirit by hanging back and tugging at the halter : in some instances it proves an excellent quietus to fatigue him by lunging in a fallow field or upon a dunghill. As soon as he has become tranquil or sullen enough to bear approach, an attempt may be made to put a twitch around his nose or his ear; not however that this is always necessary or even recommendable, for I have known colts prove more manageable without any such check. Having led him to the place where we intend to cast him it becomes a question with what means we are provided for this purpose. If we have hobbles and we can manage to put them on, they are certainly the preferable means of security; and the best mode of using them is this-Cast the horse in the ordinary way, and turn him when cast upon his off side; then pass round the near hind cannon a piece of broad web made for the purpose, or, if that be not at hand, the hobble of a side-line, and having released it from the hobble connecting it with the other legs, slip the web into the heel, and draw the foot forward and confine it against the shoulder by passing the web or rope around the neck and a second time around the leg or through the ring of the hobble, after which the web or rope may be made fast, though it is the safer practice to have it held by two men : one man will be strong enough to maintain the extension of the other three legs. A fourth man will be required at the head, whose duty, the moment the animal is cast, is to place his knee upon the neck so as to cushion it in the hollow behind the ear; at the same time he ought to raise the nose with the halter from the ground in order to prevent obstruction of the nostrils, and every time the animal struggles force the head backward, which he will find will add considerably to his counteractive power in 
keeping it from rising. But it may so happen that hobbles are not obtainable, or that they cannot be buckled round the legs of a wild and unruly colt: what is to be done in either of these cases? A rope is to be provided about thirty yards in length and two inches in diameter - a cart rope will answer the purpose very well and can generally be procured; this is to be equally doubled and formed in the middle by a double knot into a loop sufficiently large to admit the head through and hang upon the neck and shoulders with the knot turned downward after the manner of a harness-collar. The two ends of the rope coming from the knot in front of the breast are then to be passed between the fore legs, extended obliquely backward, carried round the hind fetlocks within the hollows of the heels, brought forward again on the outside, passed under the collar rope, and a second time carried backward on the outward side of. all, and extended to its full length in a direct line behind the animal: having thus fettered him it will be found that his hind feet may be drawn under him towards his elbows with the advantages of a double pulley. Prior to the pull being made it is a good preparative to place his hind feet, if we dare venture to handle them, in advance, so that less work may remain to be done by force. Two pullers are required for each rope, and the men ought to be stationed directly behind the colt as near as convenient to his quarters, it being now impossible for him to kick; and in pulling they ought to exert themselves all at once, and no less forcibly and simultaneously than suddenly, so as to take the animal by surprise : this will prevent much retrocession. The moment the animal is thrown the man at the head must knee it down in the manner 
afore-described, while the pullers are continuing to haul the legs forward: when the feet are drawn near to the elbows the pull must be steadily maintained until the rope has been twisted once or twice more round the fetlock; one rope is then to be carried backward, the other forward, and both are to be firmly kept by assistants in a state of extension. 'The colt may now be turned upon his back and propped in that position by a bundle of straw placed against either side of the body. Thus will every facility and perfect safety be afforded to the operator.

A part of the old practice consists, as a preliminary to the operation, in unsheathing and drawing out the penis in order to cleanse it and afterwards lubricate it with oil or lard; the end proposed in doing which is, I have a notion, to render the protraction of the organ more facile to the animal in the event of much subsequent tumefaction: if $\mathbf{I}$ be right in my rationale an I shall remark about the preparative is, that I doubt the fact and consequently cannot reasonably be expected to become an adopter of the practice.

The first step the operator takes, after having provided and conveniently arranged his instruments and whatever else he may be likely to require in the course of the operation, is to place himself immediately behind the animal and proceed to handle the scrotum in search of one of the testicles; for, strange as it may seem to one who has not been in the routine of such practice, in consequence of the testicles not having completed their descent, the power the cremaster has over them, and their irritability at early age, it often happens that they are not very readily found, and when found with some difficulty retained within the imper- 
fect grasp of the hand: should this prove to be the case the operator may include the testicle together with the skin covering it between the clams*, taking care however not to make use of more compression with the clams than is necessary simply to prevent its escape. The scrotum is now to be laid open by extending a longitudinal incision in the course of the inferior border of the testicle from its anterior to its posterior end; which incision may be made either with a scalpel or the actual cautery : those who use the knife aver that less inflammation ensues; those who employ the iron affirm that this increase of inflammation is more than compensated for by the opening remaining longer unclosed, and by the suppression of some trickling hemorrhage which is apt to confuse the progress of the operator. It is not a point of great importance; but, for my own part, I decide it in favor of the knife. Should the first incision have not penetrated the tunica vaginalis, this membrane is now to be divided in the same manner as the skin has been; this is no sooner done than the testicle springs from its sac, an event commonly announced by a violent struggle, during which the cromaster exerts its utmost to withdraw the testicle: of this circumstance the operator should be aware, for, unless his grasp or clams be firmly maintained at the moment, the testicle will escape and recede to the ring; and this is a slip that not only evinces a want of foresight and dexterity on the part of the operator, but is attended probably with considerable inconvenience and protraction in the operation. Not however by any means that the operator is to make any active opposition to this convulsive effort; his duty is firmly to maintain his hold and keep up

* The clams in common use have not sufficient curvature. 
a steady and obstinate resistance, patiently awaiting the remission of the struggle which will be followed by relaxation of the cremaster and will give him the entire command of the testicle. This over, the clams (which may or may not have been preparatively lapped with a broad skein of fine tow) are to be applied upon the naked cord; but they are not to be closed for compression until the vas deferens has been divided and liberated from their teeth, a precaution that will save the poor brute a great deal of suffering, and therefore one, I feel convinced, I have no occasion to inforce on any other grounds, though I might do so on the principle of its admitting a more effectual compression of the blood-vessels. Also, before the clams are finally closed the place of section of the cord must be determined. No invariable rule of direction can be given for this : if it be left too long it is apt to hang out of the wound afterwards and retard the process of union; indeed I have known the end of it swell into a fungous excrescence which greatly embarrassed the practitioner to destroy; on the other hand, if it be cut very short and the arteries happen to bleed afresh after it has been released from the clams, the operator will find it no easy task to recover it from the retraction of the cremaster. The natural length of the cord, which will mainly depend on the degree of descent of the gland, is our only guide. Having marked the place of section, the clams are to be shut sufficiently close to stop the circulation and hold the cord between their teeth without any risk of its slipping : at this time they may be fastened or not as it happens to suit the convenience of the operator.

The actual cautery employed may be either the common firing iron with rather a sharper edge than is required for ordinary firing, or it may be an iron with a 
straight instead of a curved edge and made additionally thick at the back in order to retain the heat longer: I have been in the habit of operating with the former myself, and I must confess I do not see any reason for changing it. The cautery should be heated to that degree at which it begins to turn red; irons in general in veterinary practice are used too hot, the common notion being the hotter the iron the greater the benefit conferred by it: a farrier's aphorism which the sooner we get rid of the better. The cauterization is to be made about three quarters of an inch from the surface of the clams; for, should secondary hemorrhage ensue, this spare piece of cord will be found particularly serviceable; but the cord is not to be severed by one continued application of the iron, no! reiterated searings are to be made with a quick sawing movement of the hand, and not twice together precisely in the same place unless hemorrhage require it, so as to leave the cord, when it is completely divided, with a fringe-like extremity: the withdrawment and re-application of the cautery must be regulated by the smoke arising from the searing, which may from time to time be puffed away by the operator. When the testicle has been removed, providing there be no bleeding; the clams may be very cautiously and slowly opened in order to ascertain whether the hemorrhage be completely staunched or not; should it not be, the places of eruption are to be seared again, and may be so with a fresh heated iron, or the heel of the one in use; but, if the bleeding be already effectually suppressed surely there can be no good reason for the re-application of the cautery, a practice much too prevalent, or for subsequently sprinkling and melting upon the part powdered resin: I deny the as- 
sumption that this resin renders secondary hemorhage less likely to happen, for reasons I have no time to enter into here, and I consider the practice injurious in as far as such a brittle asperous material is calculated to become a source of internal irritation. Should the spermatic artery break out repeatedly afresh after several partial cauterizations, which is every now and then the case, especially in old subjects, a new surface should be exposed by shifting the clams a little higher, and burning off the spare pice of cord : this will submit the vessel anew to the operation of the iron. As soon as the hemorrhage appears to be staunched, the cord is not. to be released at the moment, nor all at once, nor carelessly: let all remain quiet for a minutc or so, squeeze from a sponge some cold water over the parts, and afterwards with slowness and caution dilate the clams so as to liberate the cord little by little.

The operation concluded, I recommend that the animal be housed. Turning out afterwards, even supposing that the season, both from the absence of fles and the congeniality of weather, permit, I am decidedly averse to: I know very well that it is the practice of several respectable and experienced veterinary surgeons, but it is one from which I have witnessed some untoward results and therefore one 1 have reasons for discountenancing. A roomy, loose, ventilated box in my humble opinion is the best apartment for him, and green meat when it can be procured the most suitable provender; the surface of his body should be kept warm; and a purge may with prospective benefit be administered to him. For my own part, to speak conscientiously, I believe that the less that is done over and above this the better for the future welfare of the 
animal: I know that it is the common practice to "open the holes" the second day after the operation, and that there are practitioners who are in the habit of introducing digestives as they are pleased to consider them; and I am perfectly well aware that the sloughing extremities of the cords must come away and that if the openings close immediately after the operation they cannot be discharged; but, in reply to all this I say, that where the iron has been employed the wounds cannot unite by the first intention, and that they rarely or never do after the employment of the knife because they are left unconfined by suture or any means purposely that they may not. Not that there can be much harm done in simply breaking down any new adhesions that may have formed in the openings within the space of forty-eight hours; but, what I object to, is, the irresistible propensity to do something when all is doing that ought to be doing by the spontuneous operations of the parts themselves. If a healthy purulent discharge make its appearance on the secund or third day, and the swelling of the scrotum, extending more or less along the belly, be moderate, the case may be regarded as proceeding favorably; if, on the other hand, there should be no issue, the parts much and extensively tumefied, or there be no swelling at all without issue, pus is most likely confined and requires vent giving to it by insinuating the finger through the openings and detaching the lips of the wounds from their surrounding adhesions, whicl may either be caused by some inspissated blood or other discharge, or be the result of the adhesive inflammation.

There is yet another operation that has been devised for removing the testicles, and one that does not want

PART III. 
advocates: I mean that accomplished by means of the potential cautery. Some years ago certain obscure person or persons, of whose names I am not advised, obtained considerable celebrity for this operation, which they declared surpassed all others in the safety of its performance and the little subsequent inconvenience and pain experienced by the animal. The caustic they used was, and, for aught I know, remains a secret: one however has been experimented of late years and appears to answer the end extremely well. The usual method of operating is this-

Provide four fluted pieces of wood, each about four inches in length: four such pieces of elder from which the pith has been scooped, about half an inch in diameter, will completely answer the purpose. Fill the channels of them with a paste composed of oxymuriate of mercury finely pulverised and mucilage or any soft, mouldable, adhesive substance that will not affect the virtue of the caustic; and afterwards fasten the ends of them together in pairs so that they may be opened and shut to a certain limit like a pair of clams. This done, the animal is to be cast and secured in the ordinary manner, the testicle bared, and a pair of these caustic clams applied upon the cord at the determined place of section, in the same manner in which the metal clans are put on; after which the loose ends are to be brought together and tied with force enough to compress the blood-vessels of the cord and prevent the clams from slipping. The other cord being similarly served, both testicles are left dangling out of the scrotum, which is prevented from descending over them by the sticks, and the animal is suffered to rise. In this condition he is turned loose into a box. Twenty-four 
hours afterwards the testicles are to be amputated with a common scalpel below the clams, and the clams to be taken away. The parts are left then much in the same state as after the application of the actual cautery; I therefore need not say anything about aftertreatment.

This operation is said to be followed by less inflammation and its consequences, swelling and discharge, and they to be of shorter duration, than what are produced by the actual cautery; and for this reason to be safer in its result and particularly recommendable for stallions who have arrived at or passed the adult period: it is one that $I$ have not practised myself, but I have this report of it from unquestionable authority. I once made a similar experiment with potassa fusa and soft soap on an ass. On feeling the testicle an hour afterwards, for only one was experimented on, it dropped into my hand, and the animal died on the fifth day following of peritonitis. 


\section{LECTURE LXI.}

\section{On the Eye.}

$\mathrm{W}_{\mathrm{E}}$ have now the pleasing task before us of inquiring into the conformation and texture of that curious organ to which the animal owes the extraordinary faculty called vision. The parts constituting the visual apparatus being both numerous and various, I shall make a classification of them into those parts immediately concerned in the production of vision, and those which are but auxiliary to the function; in other words, into the EYEBALL and its APPENDAGES.

\section{The Appendages.}

REGARDING their several offices in a general view it will be found that the eyeball was furnished with the parts I am about to describe for the purposes of protection, motion, and abstersion; so that, as we shall discover in the course of our investigations, though no one of them can be taken away without manifest damage to the organ, they are but accessary not absolutely necessary to the operation of vision.

In viewing the exterior of the eye in man what characteristically attracts notice is the SUPERCILIUM 
or eyebrow, that ornamental arched eninence, clothed with hair, upon the superciliary ridge of the frontal bone, which forms so striking a feature and marks such expression in the human countenance, while it serves as a shade to protect the eye from descending glares of light and from foreign bodies coming in the same direction. Although eyebrows are not allowed to the horse by veterinary writers, the elevations formed by the orbital processes of the frontal bones have similar relation to the eyes to what the same parts have in man; and these eminences in addition to their common pilous coverings are furnished with many long hairs, which, though they are rather stragglingly planted, for the most part slant outward and are disposed in arches: call them therefore by what name we may, they are cvidently designed to intercept vivid rays and foriegn matters descending upon the eye.

THE PALPEBRE or eyelids are those moveable curtains in front of the eye that occupy the space comprehended within the circular ridge of the orbit. There are two of them:-an upper and a lower lid. The upper being much the larger and more moveable one, will cover a proportionably broader segment of the eyeball. Both being capable of retraction and expansion we find them in the former or ordinary state, particularly the upper, drawn into wrinkles which run in curves and have some effect in the expression of the eye: in general these wrinkles are more strongly marked in old horses. The lids are separated by a transverse aperture or slit, at the extremities of which they have angular junctions denominated the cunthi or angles of the eye: the superior or temporal angle is sharp, is situated in respect to the other in the direc- 
tion of an oblique line drawn upward and inclined outward, and has a loose and moveable attachment to the tarsal ligament; the inferior angle is extended and rounded off, and is firmly fixed to the tendon of the orbicularis. The palpebral aperture exposes more or less of the eye in different animals and individuals: in man and in the pig much of the white of the eye is seen; but in the horse and in quadrupeds in general the transparent part only is exposed, unless when the eye happens to be turned to one side. The diameter of this opening will also account for the impression made upon us that one individual has a large or full eye, another a small or sunk eye; for I believe that the magnitude of the eyeball itself will be found to vary but inconsiderably, or not sufficiently to explain this apparent diversity. The loose portion of integument entering into the composition of the upper lid is prolonged from the skin covering the forehead; that of the lower lid is derived from the face. Many short horse-hairs, but not more than three or four long ones, are planted into the upper lid, growing in the same direction as those upon the orbital prominence above; but from the lower lid stand erect six or seven hairs of considerable length, having no regular arrangement, which appear to be stationed there as sentinels to give the alarm to the organ of approaching danger; for, if by any accident one of them be touched by an insect or any foreign body in its way to the eye, the lids are thrown involuntarily into a convulsive twinkle with such alertness as almost for a certainty to shield the organ from impingement. The internal surfaces of the lids are shaped into regular concaves to adapt them to the globe of the eye with which they are everywhere 
in exact appositior. They are lined by the conjunctive membrane. The hems of the lids, the ciliary borders as they are called, are considerably firmer and something thicker than other parts, but this augmented thickness diminishes towards the temporal angle: they limit our view of the eyeball, across which the upper border swells into a curve, while the lower proceeds nearly in a straight line. Each of these borders has two facings or margins: the anterior margin is set with a row of horse-hairs denominated eyelashes; the posterior is hairless and smooth, broadens as it approaches the inferior canthus, and slopes a little inward in such manner that when the lids are closed a triangularshaped conduit is formed by their approximation, by which the tears are conducted into the puncta. The edge of the lid, the boundary line between the two margins, presents a series of pinholes, which are the orifices of the ciliary glands: they give issue to a sebaceous secretion that serves to prevent adhesion of the lids during sleep, also the escape of the tears as they run along the triangular canal.

The cilia or eyelashes are those long, fine-pointed horse-hairs growing from the anterior margins of the lids. They are generally black, unequal in their lengths, and are implanted in ranks, two, three, and four deep. They are much longer and their ranks are more regular in the upper than in the lower lid: in the former they grow thin and are discontinued altogether towards the upper canthus; in the latter the same thing happens in approaching the lower canthus. Now, it requires but little discrimination to perceive that this is the most advantageous arrangement they could have in both lids: it is obvious from the position of the head 
that light coming from above will dart upon the tem $~$ ple, while that which is reflected from the ground will be directed to the nasal canthus; consequently, the cilia are suitably disposed to intercept rays in either direction.

The eyelids in composition are cuticular, muscular, cartilaginous, and membranous; also, I may add, glandular, vascular, and nervous. These several parts and textures are most developed in the upper lid. The skin covering and composing the lids, is thin, fine, soft, and very extensible in its texture; it becomes more attenuated as it approaches the ciliary borders, around which, to a broader extent in the lower lid, it is destitute of hair. This marginal portion, which is commonly black, in some horses pied, is pierced by the eyelashes, perforated by the ciliary holes, and turns in to join the conjunctive membrane from which it can only be distinguished along the line of union by their respective colors.

The orbicularis palpebrarum consists of a broad subcutaneous stratum of fleshy fibres encircling the lids, which are most numerous and strongest in the lower lid and around the inferior angle. These fibres are attached to, indeed they may be said to arise from, through the medium of a dense firm substratum of cellular texture, the bones forming the rim of the orbit; they have also an origin from a little ligament at the inferior angle which attaches the caruncle there to the os nasi. Around the superior angle the fibres coming from above decussate those that are ascending. They are extensively inserted by cellular tissue into the skin of the lids around their borders. The use of the orbicularis is to approximate or shut the eyelids; and if it 
do this forcibly it will at the same time throw them into wrinkles: its action is most manifest on the upper lid. Although this is the only muscle employed in the closure of the lids it does not appear to be in action during sleep; in that state it seems that the lower lid is perfectly stationary, and that the upper slides into apposition with it from a natural tendency, referable to elasticity and conformation, which it has so to do when it is released from the action of the levatores, the same as what we see happen when those muscles are paralysed. Similar reasoning appears to apply to dormant animals, at least the length of time they continue asleep seems to favor it; for it is contrary to analogy and unphysiological to suppose, that the orbicularis, which at other times is seen only to be roused by volition or excitement to the eye, should maintain its action, and for such a duration, after both these stimuli have been withdrawn. In voluntary action the lower lid is seen to be raised a little to meet the other; but it recedes afterwards by its own elasticity. The fibres are so disposed at the nasal angle that in action they will also compress the lachrymal sac.

Underneath this muscle is found a dense, tough, fibrous expansion; and interposed between the two is a long, loose, cellular tissue which admits of very free motion between them. This fibrous substance is the expanded tendon of the levator palpebra superioris : a muscle that will be described hereafter. It may be traced completely over the tarsal cartilages; indeed we only lose sight of it when we come to the border of the lid.

The tarsus or tarsal cartilages are the substances imparting the firmness of feel and the elasticity to the borders of the lids which I have already had occasion 
to notice. There are two of them, a superior and an inferior: the superior tarsus is the broader and more convex cartilage; indeed they correspond nearly in shape and size to their respective lids. They are convex outwardly; concave, to clasp the eye, inwardly. They have thick and firm ciliary margins, but grow thin as they recede from the borders of the lids and end in fibrous expansions which connect them to the rim of the orbit, and hence have got the name of the tarsal ligaments. The tarsus is fibro-cartilaginous in texture; but it is so intimately united with the tendon of the levator and beset with the ducts of the ciliary glands that it can scarcely be said to be demonstrable in a separate and perfect state: in short, the tarsi are the flexible shapes upon which the other soft parts are stretched, preserving by their elasticity the form of the lids, keeping them in constant apposition with the eyeball, and serving to approximate them when not counteracted by muscular force. At this stage of the dissection we gain a view of

The ciliary or Meibomian glands. These are so many, little, white, follicular bodies, whose canals are large enough to admit a pin, vertically ranged in parellel lines like the pipes of an organ along the borders of the lids, within grooves made for their reception in the concave part of the tarsal cartilages. They are distinctly visible when the lids are everted through the thin lining membrane. They vary somewhat in calibre, but more in length : they are longer in the upper than in the lower lid. Viewed through a microscope, each row, upper and under, is found to consist of a congeries of very small spheroid bodies, every one of which is considered to be a distinct gland and to have an excre- 
tory duct. They secrete an unctuous or a sebaceous matter which may be squeezed out from the mouths of their ducts, the ciliary orifices, in taper portions resembling small white worms. The several textures we have been examining, including the lining membrane yet to be described, are all connected together by a fine cellular tissue entirely destitute of fat: adipose substance here would not only have proved burdensome and inconvenient to motion, but by accumulation must inevitably have permanently closed up the eyelids; the same as what occasionally happens from serous effusion, to which they are from their loose contexture particularly permeable.

The tunica conjunctiva is the membrane which lines the lids and from them is reflected upon the eyeball: hence the derivation of the epithet conjunctiva; it is also called the tunica adnata from a notion that it arises from or grows to the globe of the eye. To convey a familiar notion of its extent and reflections, I may remark, that if I introduce a probe or my finger between the lid and the eyeball and attempt to push it to the back of the orbit, I find that I am suddenly arrested, and that I cannot effect my purpose, try where I may, in consequence of meeting with a doubling of conjunctiva; nay, more! I find I am unable to touch any part but what is covered by this membrane. For, after it has given a complete lining to both the lids, even to their edges where it is pierced by the ducts of the Meibomian glands, and after it has given coverings to the membrana nictitans, caruncula lachrymalis, and puncta lachrymalia, it leaves the lids and becomes reflected upon the globe, to the anterior hemisphere of which it also gives a complete coating; so that it is the 
angle of reflection of this membrane that forms the boundary between the shut and open cavities of the orbit, between the tangible and intangible parts of the globe, and that prevents my probe or finger from reaching the bottom of the eye. Being the common investing and connecting membrane of such moveable parts, we find it running into loose folds in passing from one part to another, and these folds are furnished with abundance of cellular tissue. To trace its connections -along the line of the cilia it forms a union with the common skin; in passing backward it adheres closely to the tarsi, and afterwards has a loose connection with the tarsal ligaments; it gives the membrana nictitans a close covering but is thrown into folds around its base ; lastly, it adheres but loosely to the opaque cornea but is intimately and inseparably united with the transparent part: indeed, so perfectly is the membrane incorporated with the latter that the existence of such a covering has been doubted; the continuation however has obtained of late years the passport of our best human anatomists, which, with the corroboration of some remarkable circumstances connected both with health and disease, appear to have set the question at rest. The conjunctiva is not thrown into wrinkles as the skin is when the lids are opened; the elasticity of its cellular connections in general, and particularly that which it has with the adipose matter at the bottom of the orbit, which from being stretched will recede again, effectually prevents subsequent corrugation. The conjunctiva presents two ample surfaces: the adhering one is rough, lax in texture, and flocculent; the loose surface is smooth, glossy, and humid with secretion. In texture, it is no more than a finer sort of cellular 
inembrane condensed so as to be rendered firm and resisting, in which are distributed numerous blood-vessels that give it a carnation hue. There are some striking varieties however in its organization, notwithstanding it is everywhere one and the same continuous membrane : e. g. the conjunctiva palpebralis possesses numerous blood-vessels from which, as we know, it appears commonly red; the conjunctiva scleroticæ is comparatively thick and is pulpy in texture, only shows a straggling red vessel or two, and from the nature of the part it covers ordinarily appears immaculately white; whereas, the conjunctiva cornece is particularly thin and dense, and is transparent, and in a perfectly sound state has no appearance whatever of vascularity; indeed, as I said before, vessels were some years ago denied to it altogether, and their existence probably would be disputed now did not inflammation of the part occasionally afford us ocular demonstration of them.

The eyelids receive their vessels from the orbitofrontal, facial, and temporal arteries. Their veins terminate in the corresponding venous trunks. They derive their nerves from the fifth pair, and are very sensible and irritable parts.

Comparing the eye to a window, the lids may be regarded as the shutters; their uses being to exclude light and to protect the organ from all violence or accident. Light being its natural excitant, exclusion puts the organ into a state of repose, and enables it to recruit those energies which intense or long-continued vision may have weakened or exhausted. During sleep the lids are closed for this end; not however that this is absolutely necessary, for if the nervous excitability be withheld or suspended, the organ is 
equally less in a state of repose, as is the case with those individuals who sleep with their eyes open. Even the act of winking has a refreshing effect from intercepting the light but for an instant.

THE MEMBRANA Nictitans, by farriers called the haw, is a concavo-convex cartilaginous body, lodged behind the inferior canthus, between the eyeball and the side of the orbit. In a perfectly healthy condition of these parts only the cuticular margin of this substance is visible, and that preserves the line of the transparent cornea; but in a morbid or preternatural state of sensibility it is protruded forward and encroaches more or less upon the transparent part of the eye. In a detached state it approaches in figure to an extended triangle, of which the short side is turned forwards, the lengthened angle backwards. The anterior part is thin, very elastic, and is bounded by a crescentic edge, terminated by two salient angles, by which it is shaped to the inward third of the circumference of the cornea; it increases in substance but grows narrow posteriorly, and there ends in a thick, obtuse, conical point which is sunk into the adipose substance at the bottom of the orbit: thus it covers the inner and inferior side of the globe, about one-third of its whole superficies. Inwardly, it is evenly concave to adapt itself to the globe; outwardly, it is unevenly convex and clothed with adeps. With the exception of the posterior end, this body is enveloped in conjunctiva, which, though it adheres closely to the thin, so loosely invests the thick part that very free and extensive motion is admitted. The crescentic margin is edged with cuticle commonly black, sometimes pied, and this is the only part that is visible externally. 
Though it has got the name of a membrana it has a thin piece of cartilage for its base, to which it owes its shape: this cartilage is thin and pliant, becomes thicker and more resisting posteriorly, and is interposed between two tough brownish substances of a ligamentous nature, the outer of which is double the thickness of the inner.

Writers on comparative anatomy treat of this body as a third eyelid. In birds it certainly answers this purpose in the ordinary way, and in some quadrupeds too; but it appears to have been given to these animals for a purpose which the lids alone could not accomplish, or at least not perform with the same ease and effect. If you make a feint to strike the eye of a horse you will perceive that the convulsive twinkle of the lids is accompanied by the momentary sliding of the membrana nictitans over the eyeball, the same as a man would oppose his arm, or a shield if he had one, to ward off the blow : in this case therefore it affords greater protection to the organ than what the lids offer. But its chief operation appears to be one for which the lids are by no means well adapted. If a foreign body happens to lodge in the eye of a man, the lids frequently rather tend to retain than dislodge it; and, were it not for his hands, it would occasionally remain and create dreadful irritation: so it would be with a horse were he not provided with a membrana nictitans, which here serves him in the place of hands in performing an office to which the lids in a general way are of themselves inadequate. Why was the monkey not furnished with a membrana nictitans? Because he can make use of his fore paws after the manner of hands. The eye of a man therefore, as well as that of a monkey, is, ab- 
stractedly considered, an imperfect organ compared with the eye of a horse! What supervenes upon the admission of any insect or foreign substance into the eye, a rare occurrence among horses, is this-The convulsive twinkle consequent upon the accident commonly carries the foreign body underneath the lid, where it soon creates irritation and pain; tears are then shed in profusion, and the membrana nictitans during the time is repeatedly slid across the eye : in one of these efforts the irritating substance is dislodged from its place, and in the next probably withdrawn upon the convex part of the membrane, from which it is subsequently washed away by the tears. But, as this part has no muscle whatever in its composition nor any attached to it, how is it put into motion?. In this manner-The space at the bottom of the orbit unoccupied by the globe is completely filled with adeps, the globe therefore cannot be retracted without displacing some of this fat, that being, as we well know, liquid in the living subject and consequently incompressible; and as the globe, when it is retracted, is drawn with an inclination inward, it is the fat next the nose or that in which the membrana is cushioned which will give way. It is quite evident what the result of this must be. The fat is forced against the membrana, and this moveable substance is projected, guided by the eyelids and confined by the conjunctiva, over the transparent cornea, which it more effectually covers from the circumstance of the globe being at the same instant turned inward. The retracting muscles relaxing, the adeps from its own inherent elasticity slips back into its place and draws the membrana nictitans along with it.

THE LACHRYMAL GLAND. To obtain a full view of 
this part in situ the orbital arch must be removed. Underneath this process of bone we find the gland, covered by the common aponeurotic lining of the orbit, supported by the eyeball, cushioned upon the levator palpebræ in fat and adipose membrane by which it is confined in its situation. It is a pale, yellowish, delicate mass, of an irregular figure, moderately convex superiorly in accommodation to the bone, inclining to the concave inferiorly to adapt it to the globe, of whose upper and outer surface it occupies the summit. Its thickest and broadest part is turned forward ; it grows thin and narrow behind. It is a gland of the conglomerate class, being constituted of many lobules, resembling externally those of the salivary glands, connected together by a delicate and easily lacerable cellular tissue; and these lobules are themselves composed of granules, which receive the terminating ramifications of the supplying arteries; from the granules also spring the radicles of the excretory ducts, and they by their interunion with one another form a set of tubes that open upon the surface of the conjunctiva lining the upper lid, not far from the superior angle, by seven distinctly visible orifices, large enough to admit an eye-probe, whose margins are marked by slight eminences upon the conjuctive membrane. The office of this gland is to secrete the tears; and they are conveyed and poured by its ducts upon the surface of the conjunctiva, where they become diffused, partly by their own weight and partly by the motion of the lids, over the transparent part of the eye. The tears consist of a watery fluid, having a brackish flavour from some saline impregnations they contain, and from the same cause possessing slightly irritating 
properties, so that when long or often discharged upon any bare part they are very apt to occasion excoriation.

The CARUNCULA LACHRYMalis is a little black or pied tubercle, whose magnitude varies somewhat in different horses, lodged within the inferior canthus, between the eyelids and the eyeball. Only that part of it is black or pied however which is visible without disturbing the lids, and this is owing to a covering it receives from the skin, which turns in for that purpose at the canthus. Its base is clothed in conjunctiva, and this is continuous with its cuticular covering. From its cuticular surface grow several very fine, short hairs. It is glandular in its composition, being constituted of a structure which it is said may be resolved into mucous follicles: be this the fact or not, it is found to yield a mucous matter by expression after death, and it is not uncommon during life to find a little mucus collected within the canthus. It appears to serve the purpose of directing the tears into the puncta lachrymalia as they flow against it along the triangular canal.

The PUNCTA LACHRYMALIA are two small circular holes, large enough to admit the end of a comron silver probe, more distinct in the living than in the dead subject, one through the inward margin of either lid near the root of the caruncle. The superior punctum is ordinarily smaller than the inferior, and the latter is nearer to the caruncle. The puncta are the openings of two little canals, named the lachrymal conduits, that are formed within the substance of the lids. The superior of them is somewhat longer than the inferior.

They are both lined with conjunctiva, which assumes a finer texture at the entrance of the puncta. They 
take an oblique direction inward to terminate in the lachrymal sac; the superior one slanting downward, the inferior also inclining a little downward but at the same time bending forward.

THE LACHRYMA SAC is a small membranous bag lodged within the funnel-shaped bony hollow that leads into the canal in the os unguis, behind and rather below the little eminence upon the orbital ridge of that bone, which forms a very convenient guide to find it. Our English veterinarians have roundly denied the existence of this sac; and such contradictions as the one here present must exist so long as people will not give themselves the trouble to look before they venture upon description. The front of the sac is crossed by the fibres of the orbicularis, which operate in discharging its contents; and it has also a connection with the tendon of that muscle. The posterior part of the sac adheres firmly to the os unguis. It is composed of a dense, white, fibrous membrane, and this is furnished with a lining from the conjunctiva. The sac corresponds nearly in figure to the funnel-shaped hollow in which it lies: it is broad and capacious above, where it is perforated by the lachrymal conduits; but contracted below, where it is joined to the ductus ad nasum. The internal surface is uneven, and is lubricated by a mucous secretion. This sac is the reservoir into which the tears flow from the lachrymal conduits; and from which they are pressed, in the action of winking, into the ductus ad nasum: gravitation also in the ordinary position of the head may asssist their defluxion.

The Ductus AD NaSUM is a long membranous canal, beginning from the contracted bottom of the lachrymal sac, running within the brony passage through 
the os unguis, then along a canal in the os maxillare superius, between that bone and the os turbinatum anterius, to terminate at the inward and inferior part of the nostril, upon the cuticular surface there, by an oblique oval orifice, which, from being constantly open, conspicuously strikes our view whenever we dilate the nostril. The precaution of its termination upon the cuticular part was evidently taken to prevent the tears from trickling upon and irritating the membrane of the nose. In the first part of its course the duct diminishes a little in its calibre; from about its middle however it begins to enlarge again and soon after acquires its former diameter. After having emerged from its bony passages the duct makes several flexures which though inconsiderable are sufficient to render the introduction of a probe through it at this part an affair of some difficulty. The membrane composing the duct appears to be a continuation of that which lines the sac: in its passage through the bones it is strengthened by a fibrous sheath. Its internal surface is shielded from the salineness of the tears by a mucous secretion. Its office is that of conveying away the tears contained within the sac and of discharging them at the external nostrils.

The tears, as they issue from the ducts of the lachrymal gland, are dispersed over the front of the eye, for the purpose of preserving the transparent part of it humid and bright, by the operation of winking; and as they make their way to the bottom of the eye by gravitation they naturally run along the triangular canal to the inner canthus and become directed by the caruncula into the puncta, which, it is thought, imbibe them by some peculiar vital action and not by any supposed capillary attraction. When the tears overflow their 
proper channels and trickle down the side of the face, the eye is said to be watery: this is commonly the condition of the organ on the approach of opthalmia; it arises also in states of common mechanical or chemical irritation; and in the human subject weeping is induced by excessive pain, and by certain emotions of the mind, particularly grief. Augmented secretion of tears may be the effect of inflammatory action in the gland itself; but it is more frequently perhaps the result of sympathy.

THE LEVATOR PALPEBR SUPERIORIS yet remains to be described. The horse has three muscles properly belonging to the eyelids: viz. the epicramus, which was put into the cranial region; the orbicularis; and the one now before us. This is a pale, long, slender muscle, spread upon the top of the globe something after the manner of a fan, within a bed of fat, with the lachrymal gland above and the sclerotica below it. It derives its origin from the very bottom of the orbit, above and rather to the inner side of the four straight muscles of the eyeball, from the inner and upper part of the foramen opticum, by tendinous adherence. 'The inuscle broadens from its origin as it proceeds over the globe, and passing anteriorly between the lachrymal gland and conjuntiva ends in an aponeurosis which is implanted into the whole breadth of the border of the lid. Its office is to elevate the upper eyelid. The act of winking is rather the effect of alternate relaxation and contraction of this muscle than of any antagonizing action of the orbicularis : though I do not mean to deny but that the latter may slightly assist the operation.

\section{T'he Muscles of the Eyeball}

ARE seven in number: four are denominated from 
their course recti; two, obliqui; one from its office, retractor. As the straight muscles arise from the same place, take similar courses, and have corresponding actions, we may include them all four under one description. Their names are the

Rectus Superior vel Levator Oculi,

RECTUS INFERIOR vel DEPRESSOR OCULI,

Rectus Externus vel Abductor Oculi,

Rectus Internus vel Addcuctor Oculi.

These muscles are found between the globe and aponcurosis of the orbit, concealed by the fat which fills the interspaces between them. They have contracted tendinous and fieshy origins from the circumferent parts of the foramen opticum, the optic nerve taking its course in the midst of them from which they gradually recede as they approach the eyeball. Diverging a little from one another in their course, they run at nearly equal distances in direct lines across the globe to the anterior part of the tunica sclerotica; where they are inserted by short expanded tendons at opposite points into the globe. The rectus superior will elevate the eyeball; the inferior will depress it; the externus will turn it outward; the internus, inward. The simultaneous action of all four muscles will retract the globe within the orbit. The combined action of any two of them contiguous, will turn the globe in an oblique or intermediate direction.

ThE OBLIQUUS SUPERIOR vel Trochlearis traverses the inner and upper parts of the orbit. It also comes from the horder of the foramen opticum, but it takes a circuitous course and exceeds any of the recti in length. It first makes for the inner side of the cavity, running between the globe and its aponeurosis, where it passes through a cartilaginous pulley of a particular 
construction; it then turns round upon the summit of the globe, crosses under the tendon of the rectus superior, and becomes at length inserted into the upper and outer part of the sclerotica, near to the place of insertion of the rectus externus. It will give the globe a rotatory motion, in a direction inward and downward.

ThE OBLiquUS INFERIor is a shorter muscle and one probably of more power than the last-described. It has an oblique situation at the inferior and anterior part of the orbit. It takes its rise from a little pit in the os unguis, behind the lachrymal opening. Its course is oblique, outward, upward, and backward, and it thickens in substance as it proceeds. It crosses under the tendon of the rectus inferior and becomes finally fixed into the under and outer part of the sclerotica, close to the junction of the white with the transparent part of the globe. This muscle will also give the eyeball a rotatory motion, but in a contrary direction to the action of the superior oblique. To illustrate the reciprocal action of the oblique muscles and its effect on the eye, Mr. Hunter used to direct a person to look at a windmill when its sails were in motion. Both acting together they may perhaps advance the globe a little; such as appears to be the case when vision is very staring or earnest, as in fright.

The retractor oculi. In the midst of the straight muscles, surrounded by fat, at the back of the globe, we find this, the seventh muscle, one that is peculiar to animals. It arises from the edge of the foramen opticum both by tendinous and fleshy fibres. After this it becomes divisible into four portions, which are united by cellular tissue, having in the very middle of them the optic nerve. Its fibres spread outward as 
it advances, and are afterwards implanted in an expanded form into the posterior part of the globe, occupying about one-third of the superficial sphere. The retractor, as its name indicates, will draw the eyeball backward; in which operation it will be assisted by the recti when the four contract altogether. It is this retraction that puts the membrana nictitans in motion. The retractive force exertible by the eye is probably much greater than we suppose it to be: Professor ColEMAN in the course of his experimental operations some years ago for cataract, found himself necessitated to employ a counteractive force estimated at no less than $20 \mathrm{lbs}$. in order to keep the eyeball from receding. The several divisions of this muscle may act singly also and thereby co-operate seemingly with any one of the recti individually.

In addition to the contents of the orbit already spoken of, viz. the lachrymal gland, the levator palpebræ superioris, and the seven muscles belonging to the eyeball, there are, besides the globe of the eye itself, some vessels and several important nerves met with there. There is the optic nerve, sheathed in dura mater, in the middle, entering at the foramen opticum and penetrating the back of the globe; the nerve of the third pair supplying three of the recti, the inferior oblique, and the levator palpebræ, besides contributing to the formation of the lenticular ganglion; the nerve of the fourth pair running to the trochlearis; the nerve of the sixth pair to the rectus externus; and the opthalmic artery whose ramifications are distributed to the globe and the various parts connected with it; and the opthalmic vein also which returns the blood into the cavernous sinus. 
These several parts are cushioned and packed in an abundance of soft fat which serves to connect them together, and at the same time accurately fills up the interspaces. Being in a state of semi-fluidity during life this substance readily accommodates itself to the various movements of the globe, which ordinarily it bolsters forward in front of the orbit, though it is every now and then forced to one side and then drives before it the membrana nictitans. That this is one of its principal purposes is shown by there being comparatively much less of it in man. In cases of extreme emaciation part of this fat becomes absorbed, producing that appearance called sunk-eyed.

The orbit is lined with a dense, firm, fibrous sheath, having the appearance of dura mater, of which it is said to be a continuation. It has fibrous attachments to the bony inequalities and foramina at the bottom of the orbit, and by several threads is fastened to the back ur rough part of the zygomatic arch. It is thickest and offers most resistance at the outer and upper parts of the cavity where bony parietes are wanting. It serves to hold the contained parts together and to protect them from external injury; and it offers resistance to the impulse of the fat whenever the eyeball is retracted. 


\section{LECTURE LXII.}

\section{The Eyeball.}

THE ball, globe, bulb, or apple, of the eye, so variously denominated from its spherical form, may be regarded as an optical instrument of complex but singularly beautiful construction into which the rays of light are received and by which they are in their passage in such manner refracted and inflected as to be collected into focal points and thereby to represent a correct image in miniature of the object from which they are radiating.

In order that we may perfectly understand the position of the eyeball in the head, the manner in which it is sustained in this position, and the various motions it is capable of, and thereby form correct ideas of the axis and range of vision, it is necessary for us to renew our acquaintance with the orbits. The orbit is an imperfect socket formed by unequal pieces of bone coming from the ossa frontis, malæ, unguis, temporis, ethmoides, et sphenoides, in the following manner and proportions-Of the external ridge: the superior and anterior part, about two-fifths of the whole circumference, is formed by the orbital process of the os frontis; 
the inferior and anterior part, about one-fifth, by the os unguis; and the remaining two-fifths by the ossa malie et temporis, in the ratio of three to one. Internally, the floor is constituted of the orbital plates of the ossa unguis et malie; the side by that of the os frontis and by the os planum; the back parts by the ossa ethmoides et sphenoides. The socket is of an irregular figure : looking into it in front it has the appearance of being of a conoid or pyramidal form, but then on examination we find that the roof and one side are deficient, that the eyeball is guarded in those places only by bony arches, which, though they are firmly stayed and well placed to ward off' attacks in the most dangerous directions, seem to leave the organ exposed in others. This, however, is far from being the case. In the recent subject, not only a considerable part of the vacuity behind is occupied by the condyle of the posterior maxilla, but the remainder of the space, posterior to the orbital sheath, is filled with adeps: and this serves as a bulwark to the globe behind, while it freely admits of the motions of the jaw. But, what principally demands our attention here is the sheath lining the orbit. We have lately examined this and found it to possess considerable density, firmness, and resistance, and to be thickest at the external and superior parts; and we now perceive that it was made so because there it has to supply the place of bone. The socket formed by the sheath is described by some as conical, by others as pyramidal; perhaps it bears the nearest approach to the latter form, though, like other anatomical comparisons to geometrical figures, it will be found not to possess mathematical precision: for, the basis of the pyramid, instead of being equidistant at every point from the 
apex, is obliquely truncated in a direction outward and downward; to which may be added, there is a prominence of the boundary edge next the nose which contributes with this truncation to give the eye a sunken appearance on that side, while the outer part of the globe projects a little beyond the socket. The orbital ridge has a quadrilateral figure; the external side is the longest, the inferior one the shortest, the vertical to the transverse diameter being in the ratio of seven to six : the line of the internal side is interrupted by a notch. The axis of the orbit directs a horizontal line more outward than forward, more forward than downward: this line intersects another horizontal line drawn directly forward at an angle of about $70^{\circ}$. and one drawn directly outward at about $20^{\circ}$. : the inclination downward however will of course vary with the position of the head. The axis of the eyeball is not exactly that of the orbit, though it also takes a direction more outward than forward; for, in the ordinary position of the head, it is perfectly horizontal, and, in consequence of the prominence of the eye next the temple, inclines more forward than the orbital axis, probably by $10^{\circ}$.: the motions of the eye will in course vary its axis considerably and especially in the lateral direction. In fact, the eyes, placed as they are in the head, command nearly a whole sphere of vision: the horse therefore is amply provided with visual means for seeking food and avoiding danger.

The eyeball is placed within the anterior or capacious part of the orbit, nearer to the frontal than the temporal side, with a degree of prominence peculiar to the individual and within certain limits variable at his will. In front the ball is suspended by the eyelids, laterally 
and posteriorly it is slung by its seven muscles, and posteriorly it is also retained by the optic nerve; which muscles and nerve, together with its blood-vessels, are, as we have seen, enveloped in fat; and this fat forms a bolster for the globe, as has also been pointed out, maintaining it in a due state of advancement, and materially assisting in preserving its proper position and giving steadiness to its movements.

The magnitude of the globe varies something in different individuals. Its figure is a compound of two spheres of different diameters, united in front by an elliptical line. The small sphere, or rather hemisphere, projects in front from the large one, and is transparent; the large sphere, the less imperfect one, is flattened posteriorly and prominent around its sides, but is opaque: the former, from its apparent resemblance to horn, has been named the cornea, or, to distinguish it from the latter which is sometimes called the cornea opaca, the cornea lucida vel transparens. The diameter of the globe, according to GIRARD, exceeds its axis by about a line-and-a-half : (trois millimétres:) this is the reverse of the relative dimensions of the human eye, of which the axis exceeds the diameter by nearly a line.

The visual organ is composed- 1 st. of certain membranous parts, mostly opaque, called coats, investing and protecting the contents of the globe; 2ndly. of a series of diaphanous parts or refracting agents; $3 d l y$. of a nervous lining which receives a representation of the object seen and transmits the impression along the optic nerve to the sensorium.

\section{The Coats or Membranes}

ArE, the tunica sclerotica, choroides, retina, cornea, 
and the iris. The three first of these membranes form the opaque case containing the transparent parts, and are, properly speaking, the corts of the eye, being concentrically arranged one within another like the layers of an onion; the cornea is the sight of the eye, the watch-glass, as it were, fitted into this case; and the iris is the coloured partition internally between the light and dark compartments of the organ, with a hole through its centre by which they communicate.

The TUNICA SCLEROTICA, so named from its hardness or firmness, composes the white part of the globe, extending from the insertion of the optic nerve to the cornea, and forming about four-fifths of the whole superficies. Behind, it receives the insertion of the fleshy fibres of the retractor, and in front it is strengthened by the implantation of the tendons of the four recti, which have been erroneously supposed to expand over the part covered by conjunctiva and account for its shining whiteness, whence the expansion has been named the tunica albuginea; but, in point of fact, no such expansion exists, the remarkable whiteness of the part being referable to the transparent glossiness of the conjunctiva itself. In other places the surface of the sclerotica is padded with fat. Its union with the cornea is so neat and perfect that one has been said to be a continuation of the other; but the sclerotica manifestly differs from the cornea, not only in being opaque, but in its texture and organization, and also in its diseases. Moreover, either maceration or putrefaction disengages one from the other, and then it is demonstrable that the surrounding margin of the elliptical opening of the sclerotica is bevelled off for the purpose of overlapping and nicely fitting the edge of the comea 
which is also sloped off, but in the contrary direction. The sclerotica is thinner upon its nasal than its temporal side, though it terminates in front in a thickened border all round; but it is thickest at its posterior part, near the centre of which is a small roand aperture for the admission of the optic nerve : this opening is situated towards the inner and inferior part of the globe, and appears, when examined internally, as a white cribriform spot. The internal concave surface of the sclerotica is in close contact with the tunica choroides, from which it commonly receives a dusky stain, the natural effect of transudation after death: these coats are weakly connected together by a very delicate cellular tissue, as well as by blood-vessels and nerves which find their way in great numbers to the choroid coat through pinholes in the sclerotica most numerous round about the entrance of the optic nerve and in the vicinity of the corneal border in front. The sclerotica possesses this peculiarity over other ocular textures, that it retains its figure in the detached state equally the same that it does when distended and connected with other parts, and that it will regain its shape after deformity or distortion immediately the pressure (should that have been the agent) is removed; even small excised portions manifest the same elastic properties: so far is this from being the case with the other component parts of the eyeball, that, we shall find, there is but one of them but what is directly or indirectly dependent upon the sclerotica for the preservation of its form, and that one (the chrystalline lens) is unable to recover itself after its shape has once been destroyed. Some anatomists consider this tunic to be an expansion, either a laminated or an entire one, of the sheath of the optic 
nerve: whether one or other or neither be the case, we find their union to be intimate, and continuity of fibre to be the uniting medium. Its fibres, which are dense, firm, and elastic, and remarkable for their whiteness, are of the same nature apparently as those that compose ligaments and tendons; they take every variety of direction, and are so strongly interwoven and matted together that no force the fingers can exert will lacerate it: indeed, they are found to decussate so generally throughout the tunic that the opinion of its being divisible into layers seems to be destitute of anatomical evidence. The principal use of the sclerotica is to give configuration, support, and protection to the formless and delicate parts encased within it. It also affords attachment to the muscles moving the globe.

THE CORNEA, also called the transparent cornea, is the part completing the spherocity of the eyeball in front by filling up the elliptical vacuity left by the sclerotica, forming rather more than one-fifth of the whole superficies. In ordinary vision in quadrupeds (not overlooking that notable exception, the hog,) this is the only visible part of the eye; they have not therefore, according to the common meaning of the term, any white to their eyes; at least none is apparent but on such occasions as when the animal, without turning his head, looks behind him, a glance that generally betokens slyness, if not viciousness: and this, I imagine, is the origin of the vulgar assignation of vice to a horse who is in the habit of shewing the white of his eye. The convexity of the cornea, which varies somewhat in different individuals, is greater than that of the sclerotica; it being, as was said before, the segment of a smaller sphere let into the segment of a larger one. The figure 
of the circumferent outline of the cornea is horizontally elliptical; not a perfect ellipsis however, for the part turned inwards is bounded by an arc of larger sweep than that turned outwards: a remark that will serve us, so long as we remember that the optic nerve pierces the inner and inferior part of the globe, to distinguish a right eyeball from a left in the detached state. The vertical diameter of the cornea bears the same relative ratio to its longitudinal that five does to eight. Its margin is cut aslant to fit that of the sclerotica, by which it is overlapped; in this manner the surfaces of apposition are considerably broadened and their union so much the more strengthened: indeed, their union is so firm that as much resistance is opposed to their mechanical separation as to the laceration of either of their textures. Its convex surface is covered by conjunctiva, continued over it from the sclerotica, upon which its limits are marked by a black elliptical line relieved by a dusky shade: this part of the membrane however is so condensed and attenuated that it is perfectly diaphanous, a circumstance that has given rise to doubt and even denial of the continuation; the fact however is now admitted to receive indubitable evidence after long protracted maceration. At first view we might suspect that the cornea itself, being so pellucid a part, was thin and delicate in its texture; but, in point of fact, it approaches in density, firmness, and resistance to the sclerotica; for neither one nor the other are we able to rend with our hands, even with the aid of the forceps. If an incision be made across the cornea, lamina after lamina may be stripped off; and while we are doing this we cannot but notice that the surface is continually freshly bedewed with a limpid serous fluid oozing from

PART 111. 
the cellular interstices: some suppose that this watery exudation is pent in cells during life, and that its escape and diffusion after death is the cause of the filmy obscurity of the cornea which so speedily follows the extinction of life. If the cornea be macerated, it swells, turns opaque, and becomes soft and flabby, and in this condition it is very readily separable into its component laminæ, in consequence of their cellular adhesions, which in the recent eye are dense and compact, being now lax and filled with fluid; indeed, the laminæ may be felt by squeezing them between the fingers, sliding one over another: for all this however we cannot correctly determine their number, but we can find that the anterior layer is evidently continued from the conjunctiva scleroticæ. When closely examined in this state the laminæ show signs of a fibrous texture; and the density and toughness of the cornea favour this idea, as also does the circumstance of its blood-vessels being both small and scarce. In the healthy condition they are too minute to admit red blood, though red vessels are seen commonly enough in opthalmia. Nerves have not been traced into its substance, nor do its laminæ appear to be sensible, with the exception of the anterior one, which has the same feeling as other parts of the conjunctiva : this is a strong fact to show their identity. Since, then, this is a part that possesses such firmness and mechanical resistance, we must consider that it was made so tough to complete the eye-case in front; at the same time, from being transparent, it is perfectly permeable to light, and as such is operative in the production of vision:

THE IRIS, so denominated from the brilliancy of its aspect and the variety of its tints, is that part from the 
appearance of which is vulgarly assigned the color of the eye. In order to examine it we must excise the cornea, behind which it is extended, like an internal eyelid, for the purpose of regulating the quantity of light going to the bottom of the eye. Its terminating edge being fixed all round just behind the cornea, they will both have the same figure in outline; but as the cornea is concave and the iris flat an interval is left between them, deepest in the middle, and this is called the anterior chamber, to distinguish it from another space behind the iris denominated the posterior chamber. It has a perforation horizontally elliptical through its middle which forms the pupil, or what is vulgarly called the sight of the eye: the aperture is rather nearer to the inner than the outer, to the upper than lower side of the globe, and this in course is attended with a corresponding variation in the breadth of the iris at these places. The diameter of the pupil will vary, and so indeed will its figure occasionally, according to the condition of the organ and. the quantity of light to which it is exposed. The outline of the pupil, both above and below, is interrupted by several, little, black, pendulous bodies, which are the corpora nigra.

We distinguish in the iris two flat faces, and two elliptical margins. The anterior face, the veritable iris, is noted for its brilliancy, and for its color which varies in different individuals. It is a remarkable fact that this variety of color of the iris corresponds with that of the hair: bay and chesnut horses have hazel eyes; brown horses have brownish eyes; and very dark brown or black horses, eyes of a still darker dusky-brown shade. This correspondence is also observable in human eyes, but the diversity of colors of the irides of 
men is infinitely greater than what we see among any one species of animals. Cream-colored and milkwhite horses have wall-eyes; and Albinos have red eyes: in both which instances the iris is destitute of any coloring matter whatever. Both eyes are commonly alike in color and shade; though now and then it happens that one is a light, its fellow a dark eye. Upon the anterior face are visible two broad elliptical belts, the inner of which may be distinguished from the outer by being a little darker-colored; and upon the latter are discernible several plicæ, of which two are more elevated and conspicuous than the rest; the inner of these two plicæ forms the boundary line of the belts, and casts a shade upon the inner belt that gives its

- surface the effect of distance when viewed in the living eye.-The posterior face of the iris is covered with a thick stratum of black mucous matter derived from the pigment, which, from imparting to it the color of a grape, has got the part the name of uvea; and it would appear that the colour of the iris (the nature and production of which yet remain a mystery) is essentially dependent upon the uvea; for if the pigment be washed off the iris is rendered colorless. In wall-eyes, in which the uvea is wanting, the iris is white; so it is in Albinos, in whose eyes there is the same deficiency. This surface has likewise a division into two by a prominent elliptical fold in the middle : the larger one has numerous convergent striæ upon it which run in radii from the outer margin, where they look almost like continuations of the ciliary processes, and terminate in this prominent fold; the inner and lesser division is a plane oval surface, apparently without striæ, perforated in the centre by the pupil.-The 
greater or ciliary margin of the iris is embraced by the ciliary circle, and has a broad attachment to the choroid coat besides, through the continuation of the pigmentum nigrum. - The lesser or pupillary margin, rendered black and prominent by an edging from the uvea, hangs loose and floating in the aqueous humor, constitutes the boundary line of the pupil, and gives attachment to the corpora nigra.-I'he pupil, then, is nothing more than a hole in the iris, oblong or elliptical in the same direction as the cornea, whose diameter varies with the intensity of the light to which the eye is exposed. There are animals however in which the pupil does not ordinarily correspond in figure with the cornea, in consequence of its varying its relative dimensions as well as its size under such circumstances:e. $g$. in the cat, the cornea is circular, but the pupil during the day is elliptical in the vertical direction, though at night it is circular also; and, indeed, the pupil of the horse, widely dilated from amaurosis or the influence of belladonna, likewise assumes the circular figure.

All that we know about the structure of the iris, is, that it is apparently a fibrous membrane divisible into two layers, which are abundantly pervaded by arteries and nerves and thickly coated behind with uvea; and that this division of it cannot be carried through the pupillary margin, there its layers becoming inseparable. Its fibres are believed by some to be muscular, the quick and free motions of the part forming the principal grounds of their argument; for the fibres by their own admission are too minute to admit of anatomical demonstration. Professor Coleman found them well marked in the eye of a greyhound that had been long 
inured to cumning coursing in a rabbit warren. Not only however do these fibres elude all anatomical test of their nature, but they are insensible to the common mechanical and chemical excitants of other muscles, and even to galvanism.--The arteries of the iris come from the long ciliary, and are arranged so as to form two circuli arterosi upon its anterior face, corresponding to the two prominent plicæ thereon: from these, minute branches are detached which are so numerous that they of themselves appear to constitute the basis or essential part of its texture. The ciliary nerves lavish their branches upon the iris, but on account of their minuteness they defy all attempts to make out their distribution.

The motions of the iris consist in contraction and dilatation, and the dimensions of the pupil are conversely regulated thereby: when the iris is contracted the pupil is dilated, and vice versâ; the pupil however is never shut so completely as to exclude light altogether. Its motions are involuntary, excited by the presence of light, and, cœteris paribus, are regulated by the quantity admitted or the suddenness with which it is admitted into the eye. This action however is not the effect of direct stimulus, but is a secondary or sympathetic result; for light has no visible operation on the iris itself, the impression is made upon the retina: in gutta serena (paralysis of the optic nerve) the pupil becomes dilated and the iris remains motionless; and when the pupil is closed so that light cannot get to the bottom of the eye, the pupil is likewise fixed. Furthermore, the motions of the iris are influenced by the magnitude and distance of the object of vision: when the eye is intently viewing a near ob- 
ject or one very small, the pupil becomes contracted; but, if the object be removed to a distance, or it have a wide surface, the pupil becomes dilated. During sleep the pupil is contracted. After death it remains in that state, either contracted or dilated, in which it happened to be at the instant of the last expiration, and cannot by any means we may employ be re-excited to action. The efficient cause of these motions remains undeveloped.

The corpora nigra. I have given this name to the little, globular, black bodies which are attached around the pupillary margin of the iris; and I have done so because I am not aware that any appellation has been assigned them by the veterinarians of this country: GrRARD, speaking of them, says, "que l'ouverture pupillaire offre communément quelques tubercles noirs, sortes de prolongemens frangés, replies en dehors, et nommés fungus;" but the term fungus sounds to our ears so much like disease that I consider this a sufficient reason for not adopting it. By this laconic description of GIRARD's (for this is all he says about them) it would appear that they are not unexceptionably present: I have not myself remarked their deficiency unless where the pigment was wanting. There are commonly three of them, about the magnitude of peppercorns, ranged en masse along the upper margin of the pupil, something nearer to the outer than the inner corner, and the largest is placed outward-most, unless we reckon a very small one indeed which is generally found beside it. Along the under edge there are likewise three, sometimes four of them, but they are comparatively inconsiderable, not being larger than pins' heads. The corpora nigra superiora 
hang more or less over the pupillary opening in front, the corpora inferiora project just enough to interrupt the regularity of the pupillary line. I am inclined to regard them as productions of the black pigment : they have similar fringed or velvety surfaces, and appear to be resolvable into the same mucous substance, and to be continued from the uvea - to be in fact uveal excrescences. Professor Colemam has seen these bodies as large as the largest garden peas without their seeming to interfere at all with vision.

THE TUNICA CHOROIDES is the black, soft, delicate texture immediately covered by the sclerotica. It extends from around the termination of the optic nerve, by which it is perforated, in accurate contact with the internal surface of the sclerotica, as far forward as the edge of the cornea, where it ends in the ciliary circle : and the sclerotica and it are connected by a very fine cellular web, by intercurrent blood-vessels, and by the ciliary nerves. - If that part of the sclerotica in union with the comea be removed, we shall expose, immediately behind the vanishing edge of the latter, a whitish circular belt about two lines in breadth: this is named the orbicularis ciliaris, ciliary circle, or ciliary ligament. It forms the medium of union or line of demarcation between the choroides and iris; and this is the place where the sclerotica has the firmest connection with the choroides, and through that connection also an intermediate one with the iris. The basis of the ciliary circle is nothing but condensed cellular membrane; but the ciliary vessels and nerves in their course pervade it and form a sort of vascular and nervous plexus, which circumstance has given rise to some other names for it. The ligament may be stripped off altogether with the 
forceps; it allheres however with most tenacity to the choroides. Fontana has described in the human oye a triangular canal in the cellular tissue between the sclerotica and this ligament; but, for my own part, I cannot find any such cavity in the eye of the horse.The inner surface of the choroides is accurately applied in every point to the tunic internally placed, the retina; though so closely applied however they nowhere adhere, either by vascular or cellular connection. Externally, the choroides presents everywhere a black surface; internally, only the anterior parts are black, its posterior concave, about one half of the entire internal superficies, being of a brilliant variegated green tint: the black part is called the nigrum pigmentum; the green, the tapetum lucidum. The basis of the structure of the choroides consists in vascularity : arteries and veins in countless numbers enter into its composition, and these are connected together by a fine cellular tissue. Though this fact has been ascertained however, the arrangement of the vessels is a question that does not seem to this day to have been satisfactorily determined: some being of opinion that there are two strata or sets of vessels, an external one of veins, which they call the vence vorticose, and an internal one of arteries, to which has been given the name of tunica Ruyschiana; while others place the veins upon the inside. - "Les artères," says GrRARD, "distribuées à la surface externe forment une couche première, unie à une lame intérieure, dans laquelle se rendent les veines." It is perhaps sufficient for us to know that this tunic is, with least difficulty in the situation of the tapetum, separable into two layers; that these layers consist almost entirely of blood-vessels, which cross and inter- 
cross one another so as to form a vascular network very difficult if not impossible to unravel; and that its arteries come from the ciliary, and its veins pour their blood into the opthalmic vein. In order to examine the structure of the choroides it should be freed from the coloring matter adhering to it, and this cannot be done without suffering it to remain some days in water which is very apt to impair its texture. This adventitious substance is spread over both its surfaces, but the inner layer is thicker and more consistent and more determinable in its limits and disposition than the outer. The outer, the entirely black one, the veritable nigrum pigmentum, begins just behind the ciliary ligament and appears to be uniformly continued over the whole of the posterior hemisphere; the inner one, partly black and partly green, lines the dark chamber, fills up the interstices between the ciliary processes, and coats the posterior face of the iris. The colored part, which is called the tapetum or carpet, or from its brightness the tapetum LUCIDUM, is not found in the eyes of men, birds, or fishes, and in quadrupeds it is seen of different colors : in the horse, it is pea-green; in the cat, it is yellow; in the stag, it is blue. In all however its relative situation is the same: it makes a circular sweep to a certain extent around the entrance of the optic nerve; in the horse, it spreads wider superiorly than inferiorly, occupying the posterior half of the dark chamber, that part which is within the axis of vision. In a perfectly fresh eye, the tapetum is bright and its limits are distinctly marked; indeed, with pains, it may be stripped cleanly off the sclerotica, and even without staining the fingers in so doing: but putrefaction destroys its verdure and consistency, and converts the whole pigment into a thin, 
sooty, semi-fluid matter, readily diffusible in water, and which leaves a dusky stain upon the sclerotica and other parts in contact with it. The pigmentum and tapetum, apparently the same substance, differing only in color, are supposed to be a peculiar secretion from the vessels of the choroides, a secretion of a mucous nature, only that it is contained within a very fine, soft, and delicate cellular tissue by which it is held together. Mr. HunTER compared it to the rete mucosum; and there would appear not only to be a similarity in composition but also some mysterious relationship existing between them in regard to their formation; for, in Albinos, purely white and cream-colored horses, white rabbits, ferrets, \&c. in whose eyes the pigment is deficient, the rete mucosum is also wanting: this accounts for the delicate whiteness of their skins, and for the redness of their eyes, in which the blood circulating in the vessels of the choroid is seen through the pupil unobscured by coloring matter. Leaving the ciliary circle, the choroides makes a sudden inflexion inward behind the iris, and then turns intensely black; this inflexion considerably contracting the space the tunic was formerly spread over, there results a superabundance of substance, which we find to be disposed in puckers or plaits, arranged in parellel lines like radii around the circumference of the chrystalline lens : regarding the doubling and plaits together as a whole, the part is named the corpus ciliare; but the plaits by themselves are called the processus ciliares. These processes behind repose upon the soft bed of the vitreous humor wherein they make counterimpressions; the membranula corona ciliaris (which will be described hereafter) being interposed. The corpus ciliare is coated with pigment of the blackest dye, 
the interstices of the processes in particular are thickly bespread with it: this explains its remarkable blackness at this part, and accounts for the radiated circle of black stria marked upon the surface of the membranula ciliaris, after the processes have been stripped off. In order to examine the structure and connexions of the corpus ciliare the pigment should be washed off; it will then be found to be continuous at the ciliary circle with the choroides, to possess the same vascular basis as that tunic, and in fact in nowise to differ from the choroides but in its disposition and arrangement. The internal part of the choroides at the place of inflection presents to minute inspection, or through a magnifying glass, numerous delicate and faintly-marked stripes, arranged in parellel radii, which are the ciliary stria; and every two or more of these striæ unite afterwards to form a single plait or ciliary process, which runs direct to the chrystalline lens, growing broader as it proceeds, adhering in its way to the membranula ciliaris, which is behind it, and terminating with an angular point against the capsule of the lens, with which, though in contact, it has no connection whatever. The best view we can have of these processes is obtained by making a perpendicular section of the eye and looking at them through the remaining portion of vitreous humor: a broad, black, well-defined line will then be seen, showing their union with the choroides; and in consequence of their posterior edges having a greyish cast we are enabled to distinguish their interstices filled with black pigment; they are also seen to vary in length, but not perceptibly in breadth.

THE RETINA, so called from its supposed reticulated texture, is the third or innermost tunic. The 
optic nerve, having reached the inner and inferior part of the globe, pierces the sclerotic and choroid coats, and in its passage through them grows somewhat contracted in its diameter; having arrived at the internal part the nerve swells into a little, white, eminent papilla (appearing as a white spot in the living eye) from whose circumference issues in white radiating lines a medullary pulp which spreads over the entire surface of the dark chamber, sustained internally by the vitreous humor, accurately applied to the choroides externally, but having no adhesions whatever until it bas reached the corpus ciliare. In an eye perfectly recent and unprepared in any way for the examination, the retina appears to terminate on a sudden, by a defined edge, at the black circle upon the ciliary body, by adhesion there to the tunica vitrea; it is asserted however by some anatomists that it does not end but only grows much thinner here, afterwards extending almost imperceptibly between the ciliary processes and tunica vitrea and proceeding to the capsule of the lens; and this part, they say, in consequence of being much compressed, puts on the appearance of nervous striæ: whereby, they add, its connection with the iris becomes established.-During life the retina is transparent, the choroides shines through it and gives color to the pupil; but speedily after death it loses its transparency and exhibits a dead or opaque whiteness : immersion in spirits of wine produces this opacity at once and gives to the membrane a little firmness. It appears merely to be an expanded mass of nervous pulp; but, scraping the surface of it as it floats in water shows it to be resolvable into a pulpy or medullary part which becomes dispersed in the fluid, and an 
extremely delicate membrane whose texture has been found to be almost entirely vascular : the latter forming the basis or vascular network upon which the former is spread, and by which it is nourished and supported. This membrane is intimately united with the edges of the opening that gives passage to the nerve, and receives its vessels from the central urtery of the retina which takes it course through the axis of the optic nerve.

\section{The Humors}

Are three in number, viz. the aqueous, chrystalline, and vitreous; and they occupy in succession the spaces in the anterior, middle, and posterior parts of the globe.

THE AQUEOUS HUMOR escapes as soon as the cornea is punctured, and the cornea itself falls afterwards into wrinkles, showing that the tension of the one is owing to the presence and pressure of the other. It fills the interval between the cornea and chrystalline lens, insulating the iris by which the whole space is divided into two cavities, named the anterior and posterior chambers : these chambers consequently communicate through the pupil ; but they are very unequal in their dimensions, the posterior being nothing more than a very narrow chasm between the uvea and the lens, surrounded by the ciliary processes whose points may be said to project into it. The aqueous humor is a bright limpid fluid, and in its properties bears a great resemblance to the vitreous, in the state in which the latter drops from its cellular case : they are both compounded of albumen, gelatine, and muriate of soda, suspended in a watery menstruum. This humor, as well as the others, has a capsule of its own; at least, 
we infer so from analogy, or rather from the separaljility of a thin transparent lamina from the concavity of the cornea which has not the fibrous character of the other corneal laminx, and which some have been able, they say, to trace upon the iris; though no one, I believe, has seen it enter the pupil : this membrane is supposed to secrete the humor; and, if we may judge from the rapidity with which the fluid is reproduced after it has been let out, its secretion is by no means either a difficult or expensive process.

The chrystalline Lens, though a solid body, is always considered as one of the humors; it is named chrystalline from its resemblance to chrystal, and lens from its shape. It is lodged between the aqueous and vitreous humors, the latter presenting in front a hollow bed for it, surrounded by the ciliary processes and parted from the iris by the posterior chamber. The lens is inclosed within a capsule of its own, by whose attachment to the tunica vitrea it is retained in its place, assisted by the membranula corona ciliaris, This capsule, the tunica chrystallina of some, is denser, firmer, and more resisting than the tunica vitrea; it cannot be shown to have any adhesion to the lens itself, there being between them an aqueous moisture, which some regard merely as a post mortem exudation, while others consider it as necessary during life to prevent adhesion of the contiguous surfaces, and call it the aqua vel aquula Morgagni, from its discoverer. The anterior part of the capsule is thicker, stronger, and more elastic than the posterior part; though the latter is something strengthened by the membranula ciliaris, and is closely, but not inseparably, united by a fine cellular web to (and therefore is not, as some have 
supposed, conlinuous in substance with) the tunica vitrea. Successful injections show the vascularity of the capsule; and those who believe in the existence of a liquor Morgagni, suppose it to be a secretion from these vessels, which themselves are derived from the central artery of the rentina.

The lens in figure approaches more or less to a sphere; it is not uniformly spherical however, but is composed of the segments of two unequal spheres, the posterior of which has more convexity than the anterior. Though perfectly transparent, it has this peculiarity in its composition-that it is soft extemally but gradually increases in firmness and density of substance from the superficial to the central part, which latter is denominated its nucleus. It appears to be placed as it were in a state of insulation within the capsule; for we cannot detect any cellular, vascular, or other direct connection between one and the other, and we constantly find both their surfaces moist with the liquor Morgagni, which renders them so slippery that whenever the capsule is wounded the lens readily makes its escape. - The nature and composition of the lens is a subject still open to inquiry: as it is in other doubtful and obscure cases, where anatomical tests forsake us hypothesis is ready to supply their place, and, as in the present instance, now and then furnishes us with some ingenious and plausible conjectures. Dr. Young* thought that at one time he saw in the lens of a bullock's eye muscular fibres with intersecting tendons, to which he ascribed the power of augmenting its sphericity: indeed Mr. HuNTER had a notion that

"Young "On Natural Philosophy." 
it possessed a power of varying its figure, and had made some allusions to a fibrous structure. These suppositions, however, of a fibrous texture, have all sprung from examinations of the lens in an opaque and altered state: so long as it remains pellucid, though its substance certainly does come away in spherical lamina, there appear no grounds for ascribing a fibrous composition to it. Notwithstanding the physiological ingenuity therefore of these hypotheses, all that we are absohutely warranted in offering upon the subject of its composition is rather the result of chemical inquiry than any demonstrable knowledge we possess of its organization. In the living animal, it is as transparent as the clearest chrystal : after death it gradually loses its pellucidity, and more rapidly when immersed in water, by which it is converted into an opaque pulpy mass. Even if it is simply squeezed between the fingers it turns opaque : a change ordinarily explained by saying, that we have destroyed its organization and extravasated its fluids. Acids and alcohol have the same effect that boiling water has upon it; they convert it into an opaque, white, firm body, such as we see it in the head of a boiled fish. It is found to be chemically composed of albumen, gelatine, and water; and this seems to be the sum and substance of our present knowledge of its conformation. Neither blood-vessels nor nerves have ever been traced into it; nor are we certain that it is organized at all, unless we receive as proofs the ordinarily assigned tests of organization - the phenomena of growth and morbid changes.

THE VITREOUS HUMOR fills the posterior concavity of the globe, the dark chamber, nearly four-fifths of its whole interior. It is of the consistence of thin

PARTII. 
jelly, and from its pellucidity and glassy appearance has got its name. It is moulded to the form of the cavity containing it, and lies in contact everywhere with the retina, though there is no connection between them unless the ciliary artery can be considered one. Beyond the boundary line of the retina it is covered by the corpus ciliare; and in front is scooped into the form of a hollow bed for the reception of the lens.-If this tremulous mass be pricked or otherwise wounded, a transparent fluid drops from it, very like water, leaving behind a thick and gelatinous part which at length becomes condensed into a membranous substance: this residue, which contained the humor, and is said to be disposed for that purpose in small cells, varying in figure and size, is called the tunica vitrea vel hyaloidea. Opposite to the corpus ciliare this membrane is double: one part of it lines the hollow containing the lens; the other advances under the ciliary processes, for whose reception it is arranged into an equal number of radiated longitudinal folds, with depressions between them, which proceed to the border of the lens and adhere to the anterior circumference of its capsule. The portion of black pigment between the ciliary processes and these plicæ adheres to both; so that when the processes are stripped off the plicæ are seen marked with a regular series of black striæ forming a radiated circle around the lens, which appearance has obtained the name of membranula corona ciliaris for this folded portion of membrane. This being the arrangement of the divisions of the hyaloid membrane, it follows that there must exist a triangular interval or canal around the capsule of the lens, which forms the base of it, with indentations in its front side : this is 
the canal of Petit or canalis Petitianus. This canal has the breadth of the corpus ciliare, behind which it is readily inflatible. It has no communication with any other part.-The fluid which escapes from the punctured mass, though of greater specific gravity, is much the same in appearance as the aqueous humor, from which, I believe, it only differs in the proportion, not in the number or natnre, of its ingredients : viz. albumen, gelatine, common salt, and water.

The arteria centralis retince takes its course through the middle of the vitreous humor to the capsule of the lens; but we cannot discover any distinct signs of vascularity or organization in this delicate tremulous mass. 


\section{LECTURE LXIII.}

\section{On Vision.}

\section{AVING made himself acquainted with the anatomy}

of the eye-with the composition, texture, organization, relative situation and connections of its several constituent parts, the student may regard himself in the situation of an optician who possesses an exact practical knowledge of the construction of a telescope or microscope without being able to give the rationale of its operation: the latter looks through the instrument he has made, and pronounces the work to be well executed; the former is struck with the apparent simplicity of structure of the visual organ, contrasted with its wonderful powers, (with which he has been practically acquainted from his birth though he has never perhaps contemplated their sublimity until now,) but, like the optician, after he has finished his examination, still remains unqualified to expound the philosophy of vision. The reason is obvious. Light is absolutely and indispensably necessary for vision-no animal could see in a state of (were there such a one as) total darkness: the phenomena of vision are all produced through the medium of light, and consequently all op- 
tical results must have the same origin; it follows therefore, that our knowledge must not remain limited to the structure of the organ or instrument itself, but be extended to the nature of light and the laws by which it is regulated before the mind can be prepared to comprehend the theory of optics and philosophy of vision.

Various hypotheses, characterised by their respective depths of ingenuity, have been framed on the nature of light. The immortal $\mathrm{N}_{\mathrm{E} w \mathrm{~T} \text { ov }}$ taught, that light consists of subtile particles emitted in straight lines from luminous bodies in every direction with great force and inconceivable velocity. Of luminous bodies there are three classes, or, in other words, there are three sources from which we derive light: the great fountain of light is the sun, the illuminator of the universe; secondly, light is artificially produced by combustion; thirdly, there is phosphorescent light, which is either natural or artificial, and is of many and various kinds. Light derived from one or other of these sources does not appear to differ in regard to the properties we are about to discover in it; it is therefore unnecessary for us to seek for any distinction between solar and terrestrial light. Considering light as material, the minuteness of its component particles or corpuscules beggars all description, and not only sets language at defiance to convey, but baffles human conception to form, any idea of its state of divisibility: to what can the imagination compare that which is said to be considerably less than one millioneth part of one millioneth of an inch! or, that of which it has been computed that a candle while burning emits every second of time a thousand times a thousand million of times a: many 
particles as the grains of sand the whole earth contains, supposing every grain to measure one-hundredth of an inch in diameter, and the light emitted to be a component part of the burning body!

With such incredible, nay, inconceivable velocity does light move that its motion has been thought to be instantaneous; and so limited is the extent of vision upon the face of our globe that it would not probably have enabled us to detect the fallacy of this conception; astronomy however has not only furnished us with proofs that its motion is progressive, but even with data at what rate it travels. Now, it has been averred, that Flying Childers (a celebrated racer) could run at the rate of a mile in a minute; a shot from a cannon would have travelled in the time about six miles beyond the goal; the sound of the cannon whould have reached about six miles further during the minute; whereas, light! darts through a space of 200,000 miles while the pendulum of a clock makes one vibration!

I have said that light emanates in straight lines from luminous bodies in all directions : a candle placed in the middle of the room equally illumines everything around it, and the light which my eye or any object in the room receives, may be regarded as so many straight lines, consisting of series of particles of light, drawn from it to the flame of the candle, which lines are technically called rays; unless when they shoot in radiance directly from the sun, and then they are named beams or sunbeams. If a ring therefore be held between the eye and the flame of the candle, it is evident that the rays passing through the ring must collectively form a perfect cone of light, of which the eye is the 
base the candle the apex or emanating point. Supposing that the rays meet with no interruption they continue to diverge to their farthest extent ; their divergent tendency however is found to diminish in the long run in inverse ratio to the distance, insomuch that at a great distance their divergence is so trifling that it is not cognizable by the laws of optics; and so the extreme rays have been called, to distinguish them from those nearer to the source of light, parellel rays. Again, the light immediately emanating from the candle is intense and effectual; that proceeding from the same body at a distance is faint and weak: this is attributable to the rapid dispersion of the particles, and to the lengthening of their intervals, which latter, it is found, bears an exact ratio to the square of the distance.

When a ray of light impinges any body it is either reflected, or absorbed, or transmitted. By reflection is meant, the recoil of a ray from the surface of a body which it has impinged but cannot permeate: it is similar in result to the rebounding of a ball or any elastic body (for reflection is peculiar to clastic bodies) from the surface of a wall against which it has been thrown: the ball meeting with resistance recedes or turns back, not, it is supposed, from a continuation of its former force but in consequence of its elasticity. Hence, reflecting bodies are called opaque, in contradistinction to others which give passage to light, and which are therefore said to be transparent. It is by reflection of light that objects are rendered visible to us, and that they appear to us of this or that color. Metals possess the property of reflection in the highest degree, more especially when their surfaces are made smooth 
and bright: hence arises the employment of them in common lanterns. Glass is another equally if not still more potent reflector: the representations in a mirror are formed by reflection; its quicksilvered back refuses transmission to the rays, which consequently become reflected, recoil and strike the eye of the observer. Lastly, respecting reflection, it is a law, that the angle of reflection is ever equal to the angle of incidence: this is evinced when I behold an object in a mirror though neither the object nor my eye is placed opposite to it; for, it will be found, that a straight line drawn from my eye to the glass will form the same angle with its surface that one drawn from the object does. This is the principle on which the camera obscura is constructed.

Light, in impinging some bodies, in permeating others, becomes absorbed or extinct. Bodies of every composition and color possess this property in a degree; but some vastly more than others. Opaque absorb much more light than transparent bodies; and black more than any other color: white, least of all.

Those bodies, I repeat, which light freely permeates are distinguished as transparent; they are also named media, since they transmit the fluid from one place to another. In entering a transparent medium whose density is dissimilar from the one it has just left, a ray turns from the rectilinear line it was pursuing before and takes another inclination, tending either towards or digressing from the perpendicular according as the new medium be more or less dense than the former one was: the perpendicular being that ray which falls perpendicularly upon the surface of the mediunı, and one which, for that reason, proceeds in the same undeviating 
straight line in which it entered. This deviation of the ray from its former course is called refraction, which literally signifies broken; and the ray actually is bent or broken at its very point of entrance intu the new medium. The familiar illusion of immersing a stick in a pail full of water, by which the stick appears to be bent or broken at the surface of the water, and the immersed part to be more elevated than it really is, is a good illustration of this; but, raise the stick upright and it will appear perfectly straight to the bottom of the pail. It is not the stick, we know, that is bent; it is the rays of light reflected from the part under water which become broken in shooting into air, and which consequently produce in our vision the optical illusion of a broken stick: were it possible to place the eye of the spectator under water the portion of stick standing out of the pail would appear bent, but, then, the bend would be in the contrary direction. Facts and experiments akin to this one have led to the induction of two of the general laws of refraction. 1. A ray of light, in passing from a rarer to a denser medium, is drawn towards the perpendicular. 2. But, in passing from a denser to a rarer medium, it is turned from the perpendicular. To which may be added, in a general sense, the denser the medium the greater the refraction. There is yet another law which deeply interests us towards the understanding of the theory of vision. And that is-a ray impinging a curved surface of another medium will be refracted after the same manner as if it fell upon a plane placed in contact with the curve at the point of incidence: in other words, the effect of a dense transparent body of a convex figure will be to collect and unite the rays all into one common point, 
which we call the fucus or focal point; while that of at dense concave body will be to separate and disperse them. What is meant by a focus is practically shown by the use of a burning-glass, as it is commonly called : this, we know, requires to be held at a certain distance from the object destined to receive the effect, in order that the rays may all be refracted as they pass through and collected afterwards into one lucid spot; and this spot is denominated the focus from the circumstance of its being found to impart heat. Glasses having convex or concave surfaces are called by opticians lenses; and there is a variety of them in use to answer different purposes; withuut, however, inquiring what these several glasses and their respective uses are, it is absolutely imperative upon us to prosecute to a certain extent the analysis of the common lens or we cannot fully understand the optical operation of the chrystalline of the eye. The axis of a lens is determined by a right line drawn through it perpendicularly to its two surfaces, and which, when both these surfaces are convex, necessarily passes through both their centres: from which it is manifest that no lens, unless it be perfectly spherical, can have but one axis. Now, if a ray of light strike in the line of its axis, it will pass through the lens without experiencing any refraction; but, if an oblique ray impinge, it will be refracted, and, providing the lens be a convex or a plano-convex, refracted inward so as to intersect in some point the line of its axis, should both be extended, or, what comes to the same thing, so as to form a focal point with a ray coming in a similar manner from the other side: the oblique rays being refracted in lines drawn perpendicularly to tangents carried through the points of refraction. All the rays 


\section{On Vision.}

however falling upon its surface will not have one and the same focus; those which impinge farthest from the axis will meet soonest after refraction, and this effect is increased, cateris paribus, in direct ratio to the thickness and convexity of the lens. This cursory view of the nature of light and the laws of reflection and refraction will serve to prepare the mind for the consideration of the

USES OF THE DIFFERENT PARTS OF THE EYE. The contents of the globe we have found to consist of a series of diaphanous parts of various forms and densities which are freely permeable to light, and whose combined operation may be stated summarily to be, to collect the rays, reflect them in their passage, and bring them to a focal point upon the retina: the share of refrangibility each part singly possesses can only be roughly estimated until their respective configurations, relative densities and proportions, shall have been accurately ascertained; data that can alone enable us to make this calculation with mathematical correctness.

Rays of light radiating from any object of vision form cones whose apices are the points of radiation and whose bases impinge the cornea: the rays that chance to fall upon the sclerotica or any other opaque part are reflected, as are also those that strike the cornea in a very oblique direction, beyond a certain angle, and this reflection it is that diffuses the lustre around the transparent part of the eye which is so characteristic a feature of animation, and one that the painter's art can but very imperfectly imitate.

THE CORNEA serves then to collect the rays and to refract them towards the ocular axis: it is, metaphorically speaking, the glass or window of the eye, the part of the case formed to admit the light; for it sup- 
plies the place of the sclerotica in front, in containing the aqueous humor and resisting external injury. Its size and figure, and the position of the eye in the head, give the horse a commanding lateral view. The horse enjoys a much wider range of lateral vision than man, and he can direct it backwards which the latter is unable to do; this extensive and varied view serves to guide him with more certainty of success in his rambles over ample plains of pasturagre in quest of food, and must prove a great advantage tu him in the season of danger; indeed the faculty of retrospection seems to have been given him with the same design that the retroaction of the ear was, to enable him the better to elude pursuit: it is said that the hare in the chase will often turn one ear backward to gathes the sounds of her pursuers while she keeps the other presented forward to avoid perilous encounters in her course; if this be true, may she not possess the power of thus advantageously disposing of her eye-sight? That hares can and do direct their view backwards is proved by the fact of some being known in the course to run blindly against trees or posts chancing to stand in their way, and dash themselves to death. But there is an additional reason why a horse should possess retrospection; for, since his principal weapons of defence are his heels, with what degree of certainty could he have directed their stroke without this faculty?

THE AQUEOUS HUMOR maintains the convexity of the cornea, and gives steadiness and support to the iris in motion, keeping it at proper distances from the cornea and lens, and preserving the figure of the pupil: it also refracts the rays of light. In the human subject, the cornea is said to have too much convexity in some 
mancess from mal-conformation, the effect of which is to comverese the rays to a focus too early-before they have arrived at the retina, and consequently to render such persons near-sighted: I am not clear in my mind about the existence of this preternatural convexity in horses, though there are googrole-eyed staring countenances occasionally seen which might reasonably raise such suspicions, especially if such horses proved to be very shy. A man has it in his power to correct this imperfection in a great measure by the use of concave glasses; but, in horses, should it ever show itself, it must be regarded, I fear, as irremediable. The eyes of old persons commonly suffer some diminution of their general tension, the natural consequence of the permanent activity of the absorbent system through the decline of life, and this detracts more or less from the refractive force of the organ; the consequence is that these people grow long-sighted, $i . e$. the rays do not converge early enough, and therefore they require convex lenses as additional refractive agents.

THE IRIS plays a very important part in adapting the eye to vision. Being extended like a curtain through the middle of the aqueous humor, it will limit the quantity of light admitted, compelling those rays that do gain admission all to pass through the very middle, the densest and most convex part, of the lens, or permitting others to accompany them through the superficial and softer parts of that body, according as it is at the time in a state of dilatation or contraction. In a weak light the pupil becomes dilated in consequence of the contraction of the iris, in a strong light the reverse of this happens; and, if the eye be exposed on a sudden to a glare of light after having been kept closed or ob- 
scured for a time, so dazzling and irresistibly exciting is the effect that vision is altogether intercepted for the moment by the complete closure of the pupil: however, the organ speedily accommodates itself to the intensity of light, the iris again contracts, though very limitedly, and distinct vision is restored. It has also been observed, that the iris was dilated in looking at a near object, but contracted in viewing a distant one; and this observation has been brought forward to show, that the iris has the power of adjusting the eye to distances : the fact itself however has by no means been accurately examined, and the inference has turned out to be a very vague and insupportable one; for, in looking at the sun the pupil is greatly contracted, and it will be much more contracted in viewing a small than a large object, though both be placed at the same distance from the eye. Indeed, the facts altogether seem to bring us back to our former induction, and to be only reconcileable on the principle, that it is by the quantity of light that the iris regulates its motion, which is paramount to distance, though the latter will influence them when the magnitude of the object and the light by which it is viewed remain unaltered. A remote object requires more light to render it. visible than a near one, and the pupil is contracted in viewing the latter, not that it may adjust the eye to the distance, but that it may exclude the light which is now superfluous and which would consequently operate against distinct vision. The effect of light on the iris is not so striking in horses as in a man's eye: were a surgeon called on to examine the iris of the former he would pronouuce it to be sluggish in its motions. Any attempt to frame a reason for this would necessarily involve us 
in the unsettled question-What is the efficient cause of these motions? It is certainly not any inherent susceptibility to light in the iris itself, as has been imagined; for, if a strong light be thrown upon its surface while the pupil is closed, no action whatever is produced; admit the light however to the interior of the eye and the pupil will instantly vary its dimensions according to the intensity or quantity of the light. " This very naturally engendered an opinion, that the iris moved in sympathetic connection with the impressions light made upon the retina, whose exquisite sensibility, it was said, required an internal guard to moderate the light admitted; but, if this were the true explanation amaurosis ought to be accompanied by a motionless pupil, and the activity of the one ought to be regulated by the susceptibility of the other; which, though generally, is not unexceptionably the case, for instances now and then present themselves, in human medicine particularly, in which the iris is found to retain its moving faculty and activity even though the eye itself is amaurotic. This notable exception however, though it tends to increase the perplexity of the subject, does not put aside the result of all our ordinary observations, that the iris is considerably influenced by the condition of the retina and by the effect that light has upon the nerve. Independently of this physiological connection, a mutual sympathy constantly exists between the iris of one eye and that of the other: though one pupil be permanently closed and impenetrable to light yet will the iris of that eye, providing it preserve its soundness, undergo the same movements as that of the healthy eye; and this is a fact that we should bear in mind at the time that we are examining eyes. Mr. LAWRENCE has observed, 
that during sleep, in that quiescent state of the organ which accompanies the repose of the external senses and the sensorium, and the general relaxation and rest of the voluntary muscles, the pupil of the human eye is greatly contracted; and, to add to this list of irreconcileable and inexplicable phenomena, the same distinguished surgeon informs us, that he has seen two cases in which all the parts supplied by the third pair of nerves-the three recti, the obliquus superior, and the levator palpebræ superioris, were paralysed, so that the lid could not be raised and the globe was drawn to one side, and yet, in both cases, the pupil was largely dilated; not from the optic nerve being insensible to light as might be supposed, for, when one of the patients looked through a small opening made in a card, producing what may be called an artificial pupil, vision became perfect.

By what power or agency the iris moves, has not, no more than the nature of the excitation required to produce motion, been yet discovered. It was reasonable to ascribe its motions to muscularity, and it was natural to regard the fibres said to exist in its texture as the muscles themselves; but, anatomy has not furnished us with indubitable evidence on this point, and there are some strong objections to be raised against any arguments that may be built upon analogical deductions. Light, which according to this doctrine is the natural stimulus of these muscular fibres, appears to produce the very contrary effect to what stimuli do on other muscles; the stronger the light is the more dilated or relaxed is the iris; and in this same condition we find it during sleep while the voluntary muscles are all relaxed: moreover, mechanical and chemi- 
cal stimuli of all kinds, and even galvanism, fuil to excite any action whatever in it after death.

The CORPORA NIGRA, I am of Professor ColeMAN's opinion, serve as shades to the pupil; with this advantage, that they not only intercept but extinguish light. When the pupil contracts into the form of an oblong slit, leaving an aperture in the middle only, these pendulous bodies are seen to project in front of the aperture; on the contrary, when the pupil is dilated they are elevated, and so much so when the dilatation becomes extreme that they are scarcely visible. Their operation appears to me to be this - When the animal is grazing, in which dependent position of the head solar rays would be particularly annoying to him, the corpora nigra superiora are projected over the pupil to intercept and extinguish them, whereby the pupil can be kept in a state of comparative dilatation to admit light from the pasture immediately around him; with the exception of that place which he has just passed over, the vays reflected from which will strike so obliquely that they also will be arrested by the corpora nigra inferiora.

THE CHRYSTALLINE LENS is the part that possesses the refractive property in the highest degree. And the rays in their passage through it undergo a decussation which is in perfect accordance with the principles of optics: hold the lens up and view the candle through it and the candle will appear inverted, the same as it would through a common lens. The chrystalline, in fact, is an optical instrument for converging the rays and bringing them to a focus upon the retina; when therefore it is removed for cataract it becomes necessary to supply the deficiency by a highly convex glass with- 
out which the patient can enjoy no useful vision. This, after all, is but an imperfect substitute for it however; for it must be remembered that the lens diminishes in density from the central to the superficial parts, softening nearly to the consistence of the aqueous humor on one side, to that of the vitreous on the other: a peculiarity in its composition which, it is believed, serves to correct the tendency to aberration * consequent upon the sphericity of the cornea. Another advantage arising from this structure is, that the lens will transmit the rays without reflecting any of them from its anterior surface, or causing any to be reflected from its posterior side, which, according to the laws of optics, it must have done had the superficial parts possessed greater refractive force than the aqueous and vitreous humors contiguous to them : an effect that would have weakened the retinal picture. Thirdly, opticians reckon that this peculiar structure renders the eye achromatic or colorless; i. e. that it refracts the pencils of rays without decomposing them into their primitive rays, which are colored : an effect that requires considerable optical skill to avoid in the construction of telescopes and microscopes by duly proportioning the radii of the curvatures of the different lenses, and one that

* Aberration, in optics, is this-Rays of light impinging the surface of a lens will not all meet in one and the same focus, if the lens be convex, nor will they have the same virtual focus should it be concave; those rays which strike the surface of the former most distantly from its axis will converge the most, and the difference will be greater in proportion to the thickness of the glass and the extent of its spherical segments. This compels the optician to make his lenses as thin as possible ; even thereby however he cannot obtain one common point of convergence, nor is it in his power unless art can imitate the structure of the chrystalline. 


\section{Un Vision.}

in the eye must have been productive of indistinctness or confusion in vision. In fine, the chrystalline lens transmits the rays without reflection, and refracts them, whatever be their degree of obliquity, in 'such ratio to their different points of transmission that each pencil forms a focus with uniform precision, be the distance of the object of vision what it may, upon the concavity of the retina.

THE VITREOUS HUMOR appears to be for the purpose of giving sphericity to the dark chamber of the eye, in order that the retina may be spread smoothly and uniformly over its concavity, and thereby display ample surface for the representation of objects of vision: at the same time, being perfectly pellucid, it offers no interruption to the rays of light in their passage to the retina.

'I'he dark chamber I have described to be the cavity filled by vitreous humor. It may be compared to a camera obscura, the pupil representing the aperture through which light is admitted into that optical machine; for it appears no less necessary to produce vivid representations that reflected or superfluous light should be extinguished in the eye than that the room of the machine should be in a state of total darkness. The nigrum pigmentum of the choroides and ciliary processes, and the uvea, may be said to constitute the darkening apparatus; for, these parts being perfectly black absorb the rays that strike against them. This, in man, is the case with every part of the choroid surface; for, though the pigment is rather a brownish than a jet black, it reflects probably little or no light: but, in the horse and other quadrupeds the part of the pigment light and brilliant, which we call the tapetum, instead of ab- 
sorbing the luminous rays striking through the retina, reflect them back upon the membrane; an effect that, though it probably weakens the picture in a strong light and renders the eye less tolerant of such rays, produces a more distinct retinal impression in a feeble light; thus giving these animals the advantage by night, the time when they are prowling about in search of prey or food. In the horse the tapetum is a variegated pea-green, a color probably selected from its harmonizing with that of his pasture, and so establishing a congeniality between the incident and reflected rays, a circumstance that would seem to favor this particular kind of vision, and enable him to graze at night as well as by day; and this description of vision is still further promoted by the greater extension of the tapetum over the upper than the under part of the chamber. Prudently, therefore, does the benighted traveller act who commits himself to the guidance of his horse when his own sight can no longer penetrate the darkness he is journeying through; his faithful steed will pick out the road home when his rider can hardly see the animal's head before him : and this is an advantage the horse mainly derives from the reflection of the tapetum, though the comparatively large dimensions of the cornea, and correspondently dilated pupil, must also be taken into the account, in consideration of the quantum of light admitted. It is this reflection of light from the bottom of the dark chamber that gives the strikingly beautiful illumined appearance to the eyes of cats in the dark, and the sea-greenness to the pupils in horses. Those animals in whom the pigment is altogether wanting, see in the dark with still more distinctness : ferrets, who seek their prey in 
dark holes under ground, have eyes of this description. The rays then that fall so obliquely that they pass through to the nigrum pigmentum become absorbed; while those that impinge within the sphere of vision and strike the tapetum, become reflected, the more direct through the pupil diffusing through it a beautiful lustre, the more oblique against the uvea and ciliary body where they also become extinguished

THE RETINA is the part upon which the object of vision is depicted, and the optic nerve conveys the impression made thereby to the sensorium. A question has arisen, whether the retina be susceptible of impression in every part. Excepting the place where the optic nerve enters we have no evidence that it is not; but, since a perfect image can only be formed upon that part which is within the axis of vision, and since the mind is intent upon that image alone, other objects, imperfectly formed from being without the visual axis, are not recognised by the sensorium : the farther objects are painted from the axis the greater this imperfection, until rays enter too obliquely to produce any effect at all. Though the limits therefore to the admission of light are only bounded by the pupillary opening, distinct and perfect vision is confined to that part of the retina upon which the oblique rays meet in focal points with those that strike the middle of the cornea at right angles and proceed in straight lines through the centres of the pupil and lens to the bottom of the eye, which points are called the visual or optic axes. Most objects of vision being situated below the line of the head, these axes in general fall above the porus opticus; did they strike upon that place, it is said, 
they would take no effect; in proof of which is brought the following experiment-Hold two objects at arms' length in the same lateral line, near to each other, directly in front of one eye, and then shut the opposite eye; this done, move one of them in a direct line outward while the view is kept steadily fixed upon the other: it will be found that the one in motion will disappear for an instant; and this is explained by saying, that just as it disappeared the visual axis struck upon the porus opticus. Perfectly distinct vision requires that the object, as well as the organ itself, should be fixed; when anything is seen in motion, the image, though perfectly made, is of too fleeting a nature to make that impression upon the nerve which the sensorium requires for accurate and minute examination. Calculations have been made about the time of duration of the impression, particularly in regard to luminous objects of which it is the most effective and lasting: the familiar optical illusion of the circle of light produced by whirling round a lighted stick proves that the impression really does remain after the object has been removed.

Of the refractive operation of the lens the effect is, on the principles of optics, that those rays which impinge the upper part of the cornea strike the lower part of the retina, and vice versá, in consequence of their intersecting one another in their passage through the lens; it necessarily follows therefore, that the image painted upon the retina must be an inverted one. And to prove to demonstration that it is so; take a fresh cye, carefully dissect off the posterior parts of the sclerotic and choroid tunics so as to bare the retina in an entire state, over which, if it be found necessary 
to support the humors, may be laid a picee of thin oiled paper; this done, present the cornea to the flame of a candle, and a miniature picture of the candle will be seen upon the retina, burning downward. Now, what is the reason of our seeing objects in their true position, since the retinal picture is indisputably an inverted one? This has long been a problem of considerable curiosity and interest in vision. Some philosophers have contended that we really did see objects turned upside down, but that we detected the illusion by touch, and by habitual experience at length corrected in the mind the visional deception: this might seem to deserve some attention in the human subject, but it is obvious that animals must behold things reversed all their lifetimes had they no other means save the sense of touch to rectify their view; but, in truth, there is no reason to believe that infants are so deceived; and we know that persons born blind see objects in their right position the moment their sight is restored. Besides, if this were true, even those who are experienced ought to see things reversed for the first time; whereas we know that they are not thus misled. The most plausible rationale of this phenomenon, is, that it is not the picture painted upon the retina but the object itself that the sensorium takes cognizance of ; that certain sensations are excited in the brain by the impressions made by light without any reference to what part of the retina may have received them: were this not the case we should be constantly subject to mis-conception in the form and position of things; for it is quite irreconcileable with every rule of perspective and painting to suppose that objects could be represented, such as we see them, in the eye. 
There is another phenomenon in vision that has exercised the inventive genius of the philosophical world; which is, there being two eyes, and consequently two retinal pictures, why is not vision double? With reference to what has just been said in explanation of the inverted image, it may be answered, that, as it is not the picture but the object itself that is mentally seen, though two impressions are made upon the nerves but one sensation is produced in the sensorium; and this rationale has been pursued further, by ascribing the cause of only one sensation being excited to the impressions being made upon correspondent points of the retinæ; in support of which is adduced the child's trick of pushing the eyeball to one side with the finger, which we know causes double vision, arising, it is said, from altering the visual axis of the impressed eye. If this be sound theory, it may naturally be asked, why do not persons who squint see double then, since the visual axes in them certainly cannot accord? To this query it is replied, that, as squinting in most cases arises from defective vision in one eye, the other alone is used for sight; and from habit the impressions received by this eye are so strong compared with those taken by the imperfect one that the latter pass away unnoticed.

How much two eyes extend the view, and what advantages are thereby obtained I need not expatiate on ; but I would call attention to a disputed point connected with this part of our subject. It is said that in intent vision we only exert one eye, and that the object consequently is not diminished in lucidity and distinctness by closing the other. It appears to me however that this only holds true of persons who have acquired 
the habit of employing one eye on such occasions more than, or to the total exclusion of, the other; and that I can myself perceive objects plainer with both eyes open than with only one. It is argued against this, that vision then ought to be doubly strong, since the object is equally seen by both eyes. But, we do not find that double impressions excite doubly strong sensations in instances of common feeling, and why should we look for such a result in this particular sense? The loss of one eye does not enfeeble sight because the other acquires greater energy, though it much contracts the field of vision. It is said to render the conception erring; and the case of mis-judgment of distances is the one commonly brought forward to show this. All I can say on this point, is, that the best hunter I ever possessed, a horse gifted with extraordinary powers for leaping, was a one-eyed horse; and this animal carried me through a hunting season, principally with Lord DERBY's deer-hounds, without, to my recollection, making a single blunder in leaping: he had a cataract when I bought him, and therefore I cannot say how long he had lost his eye-sight.

That peculiar beauty in the structure of the eye by which it adjusts itself to the distance of the object of vision, forms a subject yet open to inquiry. The two prevailing theories of the day are rather to be considered as propositional than as superseding the necessity of future investigation. One hypothesis is, that the focal distance is regulated by alteration in the figure of the lens, which seems to involve the supposition of its muscularity. The partisans of the other theory contend that this cannot be wholly true, or clse, how could the eye preserve this faculty after the operation for 
cataract? 'They say, the organ is adjusted by the combined actions of the four straight muscles, which, by compression of the globe, lengthen the axis of vision, push the lens and ciliary body forward and thereby increase the curvature of the cornea. To say the least of this latter theory, it is a very ingenious one, and, though with the other destitute of proof, carries with it a verisimilitude that the former cannot boast of. 


\section{LEC'TURE; LXIV.}

\section{On the Diseases of the Eye.}

\section{$\mathbf{T}$}

HE diseases to which the eye of the domesticated horse is obnoxious, when compared in number with the many set down by opthalmic writers to the organ in man, are certainly very few; but there is one among them that has proved in all ages of veterinary medicine so pestilential, and that even at the present day so obstinately pursues its end in spite of all remedial measures, that this of itself, in my humble opinion, is a sufficient reason for us to become well acquainted with the anatomy and physiology of the eye, and pay more than ordinary attention to it in a state of disease. But there is another reason, not less cogent, one that is ingeniously urged by Professor Coleman in his lectures, why we ought to cultivate this knowledge, and be vigilant in the season of disease, which is thisIf a man have disease or defect of his eyes and a surgeon is only able in part to relieve him so that he regains but an imperfect vision and that vision through the aid of glasses, he is grateful and goes away satisfied; but, if a horse be brought to us whose eyesight is lost and we cannot restore it to a state of, or ap- 
proaching to, perfection, without any assistance from the optician, our art has proved of little service to the horse, and probably has been productive of consequences mischievous to his owner; for, so long as the animal continued blind, under the judicious governance and guidance of his rider, he proved himself to be still very useful; but now that he can perceive glimmers of light and imperfectly view the objects in his road, he mistakes one thing for another, shys at what he was familiar with formerly, and turns out a very troublesome servant. There is yet another motive for possessing this acquisition. The community finding how indispensable it is that the horse's eyesight should be perfect, are in the habit of consulting us on the soundness or healthiness of his eyes. But how, I would ask, is that veterinarian prepared to answer this question in all its bearings and relations who professes merely to know practically a sound eye from an unsound one? Such a man may silence the groom and very possibly gull his master; but the very first time he encounters anybody whose estimation is worth seeking, he will be set down as an ignorant pretender. And since this inquiry is a very interesting one I shall make here an observation or two concerning it.

The horse whose eyes become the subject of inspection is ordinarily placed in such a situation that light may fall upon the eye but in one direction, and to insure this being in the axis of vision, the face should not be presented to the rays (which is the common practice) but the side of the face ought to be turned with a certain degree of obliquity so as to confront the eye directly with the light: not however that this is often required by those who are accustomed to such 
inspections, nor even expedient in every case, for there are eyes that can be viewed plainer in the open light. To an experienced veterinary oculist the aspect of health is so familiar, and the ordinary imperfections so striking, that he pronounces the organ at the first glance into its interior to be sound or unsound; and here the inquiry too frequently ends: there is perhaps some unnatural appearance in the eye, no matter what or how it originated, and a certificate is granted vouching for its unsoundness. But, should the horse have been brought for the purpose of having a detailed and correct opinion given of the nature, cause, duration, and probable termination of the malady, or to be subjected to prompt and decisive treatment, then does the mere man of practice wallow in his ignorance, while the accomplished professional man unravels every link in the chain of disease, fully and satisfactorily explaining its nature, boldly and effectually administering his remedies. There is no more ready mode of qualifying oneself for this important part of one's duty, the discrimination between soundness and unsoundness, than that of accustoming oneself to the appearances of eyes in perfect health: judgment itself is but comparative, the better therefore we become acquainted with the signs of health the more nicety shall we be able to exercise in the detection of disease. In these inspections we should not depart satisfied with barely looking into the organ: we ought to compare one eye with the other; mark the prominence of the membrana nictitans; the transparency and convexity of the comea; the pellucidity of the aqueous humor; the color and brilliancy of the iris; the color, figure, and size of the pupil; the magnitude, blackness, and prominence of 
the corpora nigra ; and last but not least of all, repeatedly mark the activity with which the pupil alters its dimensions on suddenly admitting light to the eye. These are the leading features to be borne in mind in the inspection of either sound or unsound eyes: what states and appearances of parts denote health I need not recapitulate; and those that characterize disease will be detailed hereafter.

The eye is liable to INFLAMMATION, common or specific in its nature and consequences, to CATARACT, and to AMAUROSIS: but, before I proceed to analyse these affections I must be permitted to make a few remarks on some opthalmic maladies to which the horse is not subject. This may at first appear a strange and irrelevant digression; but, I must defend the charge by informing the student, that there are published accounts abroad of the diseases of the eyes of horses with authority enough attached to them to mislead the tyro; and I hold it to be no mean acquisition to us, as travellers upon the high road of fact and truth, to become acquainted with the by-roads of fallacy and misrepresentation*.

* It is very natural for a man to sit himself down in his study and imagine, since he finds the same description of mucous, serous, and fibrous textures - of muscular, glinnlular, and nervous structures in a horse's that he does in a man's eye, that the one should be affected with many, if not all, of the disorders to which the other is known to be olmoxious; but, in point of fact, the human cye, according to I). Row LEy, who has written a work entitled "A Description of 118 of the Principal Discases of the Eye," is liable to disorders exceeding in number the horse's maladies al together; and there not only exists this wide difference in regard to number, but there is une opthalmic disease to which the horse's eye is exccedingly prone which is not to be found at all in the long 


\section{Common or Simple Opthalmia.}

\section{Though no distinctions are made either in the na-}

catalogue of those affecting the human eye, nor equalled by any one of them for its irremediably destructive tendency. Here is then a memorable example to show the folly and danger of erecting a nosological fabric upon an analogical foundation. We appear to trace the signs or effects of inflammatory action, variously modified, in almost every individual texture of the eyeball and its appendages in man, and no one could rationally deny the possibility of such vccurrences among horses; but, if I am to be guided by such deviations from health as are herein seen in the course of practice, I must exclude the nominal affections I am about to notice, for which some writers have with their pens inoculated the horse.

Affections of the tarsus, in which, either from defective or morbid secretion in the Meibomian glands, the eyelids are found adhering together after having been kept closed all night, the horse is exempt. from: I do not even remember to have seen a single case of opthalmia in which this was a concomitant symptom. Of what is called fistulu lachrymalis, a very troublesome disorder consisting in obstruction of the nasal duct whereby the sac becomes distended and the tears run over the face, I never saw an instance of mysclf; though Professor Coleman mentions a solitary case in his lectures. The disease known by the name of purulent or puriform opthalmia is unknown in veterinary practice: either in the form it assumes in children, when it produces such excessive tumefaction of the lids that the surgeon opens them with the utmost difficulty, or is compelled to relinquish his effurts altogether, to gain a sight of the conjunctiva, which is found to present a wrinkled and villous surface, and to pour farth a thick yellow matter in surprising profusion; or in the contagious form in which it attacks the adlult, called the pumilent, contragious, or Egyptiun opthalmia, when the eyelids are also so tumefied that it is exceedingly irksome and painful to force them open, and a thick yellow matter is discharged over the face which will communicate the same disease if applied to a healthy eye, and which, if bold and effectual measures be not taken early, will speedily occasion ulceration and rupture of the cornea, and thus end in the total destruction of the organ. 
ture or tendency of common inflammation seated in the same textures, yet there are two remarkable points in which this affection circumstantially differs in the eyes of men and those of horses. First, as it attacks the human eye it is considered as a spontaneous or idiopathic affection, and has been so named-opthalmitis diopathica; but the affection in horses has, I believe I may say unexceplionably, some apparent or assignable cause. The animal appears to be exempt from this affection on the same principle that he is less subject to idiopathic disorders in general, not by nature perhaps any more than man, but from a less irregular mode of living, a mode that art can do little to subvert: were it possible to induce the horse to indulge in the excesses of luxurious fare, and to lead altogether such an indolent and intemperate mode of life as passes with too many of us for refinement, the same disordered states of the chylo-poietic viscera would probably alloy his pleasures, the same morbid excitement and its pernicious consequences pervade his system as is never absent from theirs who spend their days so contrarily to the designs of Nature, so ruinously to themselves. Secondly, robust persons whose habits of life dispose to plethora are especially liable to an inflammation attacking at one time both the external and internal tunics of the eye-an opthalmia characterised by a general scarlet redness of the organ; by chemosis; (a deposition of lymph underneath the conjunctiva ;) by violent pains in the eye, shooting through the head, and often by a sense of the globe, from actual enlargement, being too large for the orbit; by a general tumefaction of parts ; and by the inflammatory fever consequent upon such violent local symptoms. In horses, on the other hand, the seat of common inflammation is the conjunctiva: 
umless in an event of direct injury to the parts I do not remember to have seen a single case of common internal opthalmia.

The seat then of common opthalmia is the conjunctiva, and its causes are external; its nature, on the principles of inflammation, is simple, and its svmptoms are few and characteristic. The principal symptom is redness, by the extent and intensity of which is mainly to be reckoned the violence of the attack; for it may vary in degree from simple vascular congestion, a condition denoted by a vivid carnation hue of the palpebral conjunctiva and the appearance of some red vessels upon the sclerotic, to the state of acute inflammation, evinced by the bright scarlet tint of the lining of the lids, by a thick vascular network covering the opaque cornea, from which numerous red vessels shoot into and obscure the transparent part: these states of the membrane are only to be correctly estimated by evert. ing and closely examining the lids. Chemosis is rarely or never seen. There are in general no signs of any considerable pain: the animal feeds as usual, seems undepressed in spirits, and appears to be unaffected constitutionally; a circumstance that harmonizes well with the yielding texture of the membrane, as well as with the little pain felt by ourselves when similarly affected. There is commonly a flow of tears over the face, attributable to participation in the increased action by the lachrymal gland. The eyeball itself being free from inflammation, aversion to light is not one of the primary symptoms, though it may supervene as a casual one should the ocular vessels sympathize with those of the lids; notwithstanding that, however, the lids will continue contracted and the haw protruded so. 
long as any cause of irritition remains; a circumstance that is apt to induce us erroneously to think that the eye is also affected. Vision is impaired only in the most violent of these affections, when the transparency of the cornea is diminished or destroyed by vascular plethora or the effusion of adhesive matter. The upper lid being ordinarily the seat of injury may be more or less tumified, and such a case may end in abscess of the lid; but these parts seldom or never swell spontaneously.

In regard to the causes of common opthalmia, I have already averred that they are local and mostly apparent, not constitutional : Professor CoLeman and our best modern veterinary writers all concur in this etiology; and I may add, that the disease would never probably have appeared in print in any other form had the subject not fallen into hands that have shown themselves unqualified by experience to pen any very correct or useful information about the matter. The ordinary exciting cause is mechanical irritation: a blow from a whip, a bite from another horse, the insinuation of a hayseed or other irritant underneath the lid, are the common excitants; though it rarely happens that foreign matters lodge in the eye, the consequent flow of tears and the reiterated action of the membrana nictitans being commonly effectual in their removal.

The treatment of these cases is in general very simple. Many of them get well spontaneously after the irritant has been removed: others have an inflammation excited which requires immediate and active antiphlogistic measures, such as will suggest themselves when we come to detail the treatment of specific opthalmia. Should there be present any signs of the 
retention of the foreign body, ine most likely situation to find it, is the concavity of the upper lid; by elevating which from the globe or everting it by means of a probe, we shall probably discover a hayseed or particle of dirt sticking in the conjunctiva.

I may remark here, that it every now and then happens that a horse gets his eyelid, commonly the upper one, torn from a bite or from hinging it in the point of a nail or hook that may chance to be fixed near him in the stable. I have had two or three such cases, in which the border of the upper lid holding the cilia was so rent in the longitudinal direction that it hung merely by a strip of skin over the front of the eye. These accidents afiord the veterinarian an opportunity of displaying a skill and neatness in the use of the needle, which may turn out no little to his credit. The torn lid requires to be nicely adjusted and sewn up with a small needle and a single thread; and the sutures should be cut and removed twenty-four hours afterwards. The part may be kept wet with cold water; unless the horse be very shy about the head, and then this had better be dispensed with. Should tumefaction of the lid and redness, \&c. of the eye ensue, the case must be treated as one of simple opthalmia.

\section{Specific Opthatmia.}

THе moon-blindness of the farrier's nosology, so named by them from a belief that its relapses were influenced by the changes of the moon and from its almost certain termination in blindness, is the specific opthalmia of our veterinarians; but what the latter mean by specific, I have not been able to make out, unless it be used as an epithet to denote that this is a 
disease whose nature or theory is not understood: at least such appears to be its interpretation in the instance before us and in glanders and farcy which are also called specific diseases. Professor ColEMAN, who at an early period of his professional career made this disease the peculiar object of his investigations, has put to flight the incorrect accounts in which its medical history was involved together with the absurd and mischievous practice grounded thereon, and he has set the subject in a light reconcileable with that of modern pathology; but, after all, though we may have got rid of palpable absurdities, have we advanced in our knowledge of its nature or devised any effectual mode of treating it? I fear not; however, let the sequel of this account be the answer.

This disease, it would appear from the ordinary account of the case, generally makes its attack at night. The groom discovers in the morning that the horse has got a "bad eye," and he seldom fails to report either to his master or the veterinary surgeon in attendance, that the animal must either have received a blow or some foreign matter in the eye in the course of the night. And indeed the eye itself betrays appearances externally that seem to favour this fallacy of the groom's ; for the upper lid droops, giving the organ a weak or gloomy aspect, the haw projects, and tears flow over the under lid and bedew the cheek: but, when we come to inspect the interior of the organ we find the cornea so dull that the iris can hardly be seen, and the eye so impatient of strong light that considerable efforts are made by the lids and haw in opposition to any attempts we may make to distract the lids by force. The most effectual mode of obtaining a full view, is to turn. 
the head from any direct or strong light, and then the animal will open the eye sufficiently to admit of inspection. The pupil will be seen more or less narrowed, to contract with unusual celerity and force when turned to the light, and to have a hazy aspect through the dull filmy cornea; the iris also will have greatly suffered in brilliancy and perhaps will show some partial or a general appearance of discoloration. As the disease proceeds the cornea continues to lose its lustre and pellucidity until we can no longer distinctly see through it, which may be the consequence of its increased vascularity or of some interstitial effusion; even the aqueous humor, I believe myself, grows turbid, so that from both these causes we can obtain but a very imperfect view of the iris: there are cases however in which the iris turns of a dead amber hue, from a coating of adhesive matter, before the cornea becomes oloscured. The corpora nigra also after a time lose their jetty blackness, and the pupil becomes so contracted that we can hardly see through it at all. These internal marks of disease are attended generally with conjunctival inflammation : in some cases the membrane becomes intensely reddened, red vessels clothe the opaque and crowd around the margin of the transparent cornea across which numbers of them shoot in serpentine lines; in other cases the conjuntival affection is less violent. It is to the tumid condition of this membrane that the fullness and tenderne's of the lids and the apparent enlargement of the haw are owing, and that the eye itself seems shrunk and more retracted than under circumstances of health. In the more advanced stages hypopyon is often present : and the case may, I am told, terminate in suppuration of the whole wlobe and consequent disruption; but I have 
not met with the occurrence in my own observation. Light opaque spots are occasionally seen upon the corpora nigra, showing that they are also participators in the morbid alterations. But the common ending of all this inflammatory disorganising action is cataract: as the inflammation abates the iris gradually contracts again, disclosing a hazy greyish pupil which would appear to result from the effects of disease in the substance of the lens, and which we know practically to prelude to a cataract; for, I repeat, ultimately, almost all these cases proceed to the formation of cataract. The pupil at the same time may or may not become contracted and rendered a fixture from adhesions : very commonly, where the other eye is sound the pupil of the cataractous one moves in unison with its fellow. The iris is more or less permanently altered in aspect and color. The cornea may have recovered its pellucidity or be clouded with a white and permanent inter-laminal deposition. These are in general the only visible alterations of structure; but it seldom happens, I believe, that the retina and vitreous humor, not to mention other less important parts, are free from organic impairment. Occasionally we meet with eyes which are nothing but bulbous masses of disorganization and alteration of structure. The cataract perhaps has been thrust through the pupil into the aqueous humor; or it has driven the iris before it, and that has grown to the transparent cornea; perhaps the vitreous humor too has made its escape into the front chambers, whereby all the parts are so cemented together that scarcely a vestige of healthy aspect remains. In some cases I have found the lens in the dark chamber, floating about in the vitreous humor; and in others, both the lens and its capsule on examination 
have proved to be partly converted into bone. But to particularize the infinite varieties of states and appearances to which the organ is reduced by this destructive malady, would far exceed my present limits and do so to very little purpose.

Some writers regard the conjunctival affection as the principal, others as the primary disease. To the former it is scarcely needful to reply. The latter, I am inclined to believe, rest their opinion upon the conspicuousness of the more determinable sign of inflammation, viz. redness, in that part, beyond other signs perceptible only on close and accurate inspection in the interior of the eye. But, the irritable iris, the contracted pupil, and the general dullness and muddiness of the transparent parts, together with their subsequent change of color and aspect, are symptoms that denote morbid action within as strongly as redness and swelling do without the organ : it argues therefore, to say the least of this opinion, a narrow view of the case to reason upon such slender premises though they be undeniable facts. If the disease were primarily conjunctival why should it so invariably extend to the globe? Common inflammation we know hardly ever does so. And why should not the disease destroy also the texture of the conjunctiva if that be its primary or principal seat? So far from doing this however is it, that the membrane we know seldom or never sustains any injury. It might with infinitely more reason, in my opinion, be designated iritis than considered either principally or primarily as an opthalmia conjunctivœ.

It must not be inferred from the description here given of its ordinary or regular course that the disease. proceeds rapidly and without intermission to the total 
destruction of the organ; on the contrary, it is comparatively tardy in its progress, and effects this final destruction by relapsing after considerable intervals of apparently restored healthy action: the paroxysm commonly continues for a month or six weeks, and then subsides altogether; leaving the eye, should it have been the first and not a very violent attack, to appearance unimpaired, only that it may evince rather more susceptibility of light than the sound eye, shedding perhaps a tear or two and twinkling a little when suddenly exposed, and having its haw rather more irritable and prominent than the opposite one. The disease is seldom seen in both eyes at the same time: a paroxysm will run through its course in one eye, and in a month or two afterwards a fresh attack will show itself in the other; or, should that not prove to be the case, almost to a certainty a relapse will invade the eye first attacked; passing through certain inflammatory stages, and afterwards leaving the organ more or less impaired in structure and of course in function. Its ordinary mode of proceeding is to attack alternately first one eye and then the other until both have experienced such morbid alteration of structure, the most conspicuous and common mark of which is cataract, that the animal is left permanently and incurably blind. This general and complete disorganization is ordinarily followed by a cessation of the paroxysms; not invariably however, for there are cases in which they continue to return even after vision is totally lost. Rarely both eyes, I repeat, show this disease at the same time; but I have seen many cases in which I have not been able to detect it in the opposite eye, though it has pretty regularly relapsed in and ultimately destroyed the other. In short, in regard to the violence 
and duration of the paroxysms, the length of the remissions, and the relapses and alternations, the disease is not reducible to any known laws, either physiological or pathological.

More or less lingering febrile action invades the system during the continuance of the paroxysm; but it is too mild seemingly to be productive of any indisposition, for the animal retains his appetite and spirits, though at the same time his pulse denotes febrile disorder, and his secretions and excretions perhaps manifest the same indication : should he be kept at work however during the opthalmia an inordinate disposition to perspire may probably be remarked, together with consequent dullness or unusually early symptoms of lassitude. If it be a local affection therefore it is one that makes some impression upon the system at large. But, Professor Coleman views it as a constitutional disorder; and I think I shall be able to show that this opinion, so far from being erroneous, is one that we ought to keep steadfastly in view inasmuch as it may be regarded as a beacon to direct us to the discovery of its origin : it has certainly taught us practically a pretty certain mode of warding off its attack. Some of our modern practitioners are in the habit of drawing an analogy between this disease and gout, and so they call it "the gouty opthalmia." These disorders assuredly both very commonly make their attack during the night, and both disappear after an uncertain duration, and return at irregular intervals; but, here I apprehend the comparison (if such it can be called) must end : so far as we are acquainted with their etiology, they arise from very different causes, and are seated certainly in very different parts; while one is in a great measure controllable 
by art, but the other pursues its course with but little variation or intermission in spite of all our boasted efforts.

In the course of his researches into the origin or production of this opthalmia, Professor Coleman from extensive observation was led to believe that the same contaminated atmosphere which is known to engender glanders and farcy, sows the seeds of disease in the present instance. Horses running wild and breathing the untainted open air are never thus affected, "at least, I never saw such a case," says the Professor; and of those that are domesticated, farm and coach and post horses are the common subjects of opthalmia; and so were formerly the cavalry horses, when they stood in ill-paved, ill-drained, unventilated stables. Some writers suppose that it never attacks mules or asses; but this is a mistake: the mules belonging to the army in the Peninsula were quite as often the subjects of diseased eyes and glanders and farcy and mange as the horses were; indeed, it struck me forcibly, at the time I was serving with the British Artillery there, that the mules especially suffered from malignant diseases contracted in ill-constructed filthy stables. Shall we say, by way of a summary rationale, that the effluvia arising from the breath, perspiration, urine, dung, \&c. generate an animal poison, and that this, being in a gaseous form, is diffused through the atmosphere of the stable, and with it carried into the system through the medium of the air-passages, skin, or alimentary canal, most likely by the first but possibly by all of them, wherein it breeds disease in the circulating fluids, which breaks out, under a local form, in the lungs, skin, nose, eyes, \&c. ? or, shall we say, that these parts are affected lo- 
cally in the first instance and subsequently contaminate the system? Let us postpone the consideration of an answer until we come to include glanders in the same family of poisonous influences. Professor Coleman adduces as proofs that it is not a local affection.-1st. The constitutional derangement, which he says may often be traced to the digestive organs and skin, and is in some respects unlike simple irritative disorder. 2dly. The character of the inflamination, which is neither so acute nor so rapidly progressive as simple opthalmia often is, and yet the one readily yields to proper treatment, while the other obstinately continues its course. $3 d l y$. The eye may be locally exposed to volatile ammonia or any animal effluvium we can obtain by artificial means, and no such effect will be produced.

The most rational indication in the treatment of a disease is that founded on the solution of its causerestimatio causæ sæpe solvit morbum, is a maxim which it would be well for those veterinarians who are so given to stumbling upon cures to have their prescription-books indorsed with: it might save them much fruitless labor and anxiety and perhaps no little credit in the course of their well-meant but ill-judged investigations. It may be said, that we have skipped over the proximate cause of this disease. Had I anything satisfactory to offer upon the subjct I should cheerfully embrace this opportunity; but as I have not, I must leave it in the hands of those who gave the opthalmia the epithet specific. It is certainly a species of inflammation, and is apparently constitutional in its origin though local in its consequences; we may therefore betake ourselves to the adoption of antiphlogistic means, and apply them constitutionally 
as well as locally, though we shall fail to make that impression which we do in simple phlegmon, and ultimately have the mortification to learn, that our efforts, though they may have delayed, have not prevented the fatal issue, permanent and irremediable blindness. It is the common practice to draw blood, and now and then we appear to do so beneficially: the evacuation should not be sparing, and the jugular vein of the same side as the affected eye will be opened with the concurrent advantages of local and constitutional depletion. The temporal artery is sometimes chosen, but in my opinion without prospects of equivalent, effect, since none of its branches run to the eyeball. The angular vein is opened with still less benefit. Blood may be locally drawn also by leeches and by scarification: the former, generally speaking, are too expensive for veterinary purposes; the latter may prove beneficial in simple opthalmia when there is considerable vascular turgidity of the conjunctiva, but cannot be expected to do much good here. But to show how very ineffectual the abstraction of blood occasionally proves, I may state here, that Professor Coleman, after having drawn as much blood as the system could withstand, has placed ligatures around the carotid and facial arteries; nay! he has even taken the pains to excise from the front of the eye one by one the red vessels that were seen shooting from the opaque into the transparent cornea, and seared the parts around the line of separation afterwards, and yet this has only put a temporary check to the inflammation; after a short lapse of time the paroxysm has returned in all its former violence and obstinately persisted in its destructive course. If 
bleeding do mitigate or shorten the duration of the paroxysm, and I conceive that it generally does so myself, it is much more efficacious in the early than the late stages; and in order to derive the full effect we must draw blood until we have sensibly diminished the force and frequency of the action of the heart: this is the only chance we have of bleaching the eye by venesection. Doubt seems to hang over the practice of purgation. I have certainly seen purges relieve the vascular tension, particularly in the early stage; but I must add, that I have occasionally looked in vain for the same apparent relief: Professor Coleman informs us, that he has attended cases in which the paroxysm, though abating at the time, was evidently renewed by purgation; and he believes that the debility consequent on this operation is likely to pre-dispose the animal to a fresh attack. I have often remarked, especially towards the decline of the inflammation, that the eye became considerably clarified by blisters, applied upon the temples or cheeks; they are not very cleanly remedies unless one be washed off before another be applied, and then there can be no such objection made to their use: I do not advocate the farrier's practice of rubbing a liquid blister indiscriminately over the eyelids and parts adjacent; it stiffens and disfigures the lids, and runs into the eye, and often aggravates instead of relieving the disease. Setons in the vicinity may be productive of some benefit in the chronic stages. Professor Coleman has exhibited calomel and the hydrarg. oxy-murias, and I have pursued his steps, but without the desired effect: the mouth may be rendered sore in the course of three or four days, so sore that the horse will cud his 
food, and this effect may be carried to salivation, and consequent starvation from inability to masticate, and yet the disease continue unabated and apparently uninfluenced. Various tonic and other medicines have been administered at random in hopes of drawing a prize: but they have one and all tarned out blanks. The cupri sulphas was started as a specific when I was a pupil at the Veterinary College; but it has proved to be only "a nine days" wonder." I have made trial of the liquor arsenicalis, the sulphate and subcarbonate of iron, and various preparations of barytes; they have all however failed to arrest or even check its progress. The advocates for the locality of this affection, those who build their hopes of cure upon various eye-powders and eye-washes have been equally foiled in their attempts: I do not mean to deny the occasional utility of them as co-operative with constitutional measures, but of themslves they are next to valueless. The vinum opii and the liquor plumb. subacetat. dropped into the eye will often afford temporary relief, even in the primary stage; but the astringent metallic solutions, particularly those of zinc and copper, should be studiously abstained from so long as any active inflammation continues. Simple, poppy, and chamomile fomentations are recommended to us by medico-veterinary writers: they are very harmless remedies, but the evil is, they are occasionally very teasing in their application; for a horse is that refractory sort of animal that, when he has a sore eye, has no notion of holding his head still to have it played with. He may probably without much trouble permit his eyelids to be wetted occasionally with cold water or the lead wash, and I believe that will answer 
the purpose quite as well. Light and air being stimulants to the organ in health, it nust undoubtedly ought to be shielded from both in disease; and this is a part of the treatment commonly too much neglected: the animal should be placed in a dark but properly ventilated stable, or winkers or shades ought to be worn by him: blinds are apt to do more harm than good by confining a heated atmosphere around the eye.

By the judicious employment of some or all of these means we commonly do, or are apt to think we do, hasten the departure of inflammation and ward off or mitigate some of its consequences; but an experienced practitioner is too well aware of the liability and formidable certainty of a relapse to repose any faith in the duration of a truce so obtained; and therefore his ordinary advice to the owner is to dispose of his horse during the interval of remission. Knowing, therefore, as we do, that such horses are continually changing owners, it is a qualification of the first importance to us in practice to be able to discriminate between an originally sound eye and one that has been visited with this malady: vision may possibly be unimpaired in the latter, but we are not to suffer our opinion to be lulled to rest upon such a slippery pillow as this. Any single one of the following appearances may, unless its origin can be satisfactorily traced to simple opthalmia, excite our suspicions; but a combination will certainly warrant an unfavorable prognostic-A sunken or gloomy aspect of the eye altogether, compared with the other; prominence of the membrana nictitans; vascularity or pinkness of the conjunctiva; a watery state of the eye ; dimness or cloudiness of the cornea, particularly around its margin; dullness or discoloration of the iris; cor- 
pora nigra yellowish or whitish or spotty; pupil smaller than the other, perhaps hazy or milky, or containing a minute white speck in its centre, which is an incipient

\section{Cataract.}

As opacity partial or complete of the lens or its capsule or both, is called a cataract: and this is one, and the most common one, of the effects of internal or idiopathic opthalmia. A grey or white speck is first perceivable in the very centre of the pupil which slowly and gradually enlarges until it quite blocks the aperture: vision grows less and less distinct during its formation; but, even prior to the speck being visible it is found that human vision is something disturbed. The growth of the incipient speck during the time that the organ is suffering from opthalmia is commonly rapid; but, as soon as the inflammatory action has subsided its progress becomes comparatively slow, and now and then it appears to remain quite stationary. For, as soon as cataract has formed the inflammation generally begins to decline, and the eye seldom receives another attack: there are however, as I stated before, some exceptions to this. And it is also, I believe, an ascertained fact, that, not only does the inflammation permanently leave the cataractous eye, but, should the other have remained free from the disease it is in less danger of being attacked; under such circumstances therefore a cataract is hailed as a favorable omen: this is explicable seemingly on the principle of sympathy which is known to have great influence in opthalmic pathology, and the circumstance has been considered weighty enough to recommend the artificial destruction of one eye in disease to preserve the other in health. 


\section{C'retaract.}

It is found that persons who have imperfectly formed cataracts see best in weak lights, the pupils being then dilated and giving admission to more rays through the circumferent and unobscured parts of the lens: no doubt, the same thing happens with horses, but when once their vision becomes confused they grow so shy and troublesome that they would prove more serviceable perhaps in a state of total blindness. The presence of cataract in the eye of the horse is not so manifest as in that of man; in the latter the defect is clear and determinable, in the former it is often hazy and has occasionally given rise to dispute of its existence even among professional men: the rationale of which is, that the natural French-greyness of the pupil of the horse makes but a weak contrast with the greyish white speck when compared with that formed by the dark pupil of a man. In order to descry an incipient cataract (for one fully formed is seen plain enough) view the eye in various lights and directions, compare the pupils, and if one appear smaller than the other look very narrowly into the centre of the former, and there will probably be perceived a speck scarcely larger than the head of a pin: but, should no speck be discernible and yet the pupil be small and have a hazy aspect, examine the activity of the iris, the result of which examination, though it must not singly be considered as determinate, may serve to increase your suspicions, and, coupled with other appearances and considerations, lead to a pretty correct conclusion.

Cataract may either be capsular or lenticular; or it may be both, and then is termed mixed; and a lenticular cataract may be firm, fluid or milky, or caseous in consistence: the first is the common variety among 
horses. Thongh there may be other causes for its production in the human subject, in whom cataract, it is said, may be of scrofulous, arthritic, venereal, hereditary, congenital, or local origin, I believe that it will be found to be invariably in horses one of the consequences of opthalmia; for in cases where no signs of increased action have attended its apparent formation, they may be generally discovered to have existed at no very remote period preceding it. I do not mean to assert that it never happens without inflammation: I have heard, and so far I believe, that it does; but it certainly is comparatively a very rare occurrence. Since we are professedly ignorant of the structure or composition of the leus, since we cannot even take upon ourselves to say for certain whether it be an organised body or not, it is not reasonable to expect that we should be able to unfold the nature or formation of cataract; but it certainly appears, at least in horses, rational to view it as an effect of inflammation, and having from facts and circumstances come to this speculative deduction, here our knowledge of the subject may be said to end*.

* In the human su'jject, cataract, it is said, has been cured by internal remedies. Be this worthy of credit or not, there have certainly been well-authenticated cases of spontaneous cures happening in various ways: surgeons however at the present day place no reliance upon these doubtful and casual results; they have recourse at once to an operation which has for its ohject the removal of the opaque body out of the axis of vision. There are threc modes in practice of effecting this. One, termed couching, consists in depressing the lens into the dark chamber, where it cannot interfere with vision. This operation is performed with a needle of a particular make, which the surgeon introduces through the sclerotica, about two lines distant from the margirs of the cornea, and one line below the transverse diameter of the globe; he directs its point 
Possessing a smattering of these proceedings, persons often come to us and ask, why such operations are

to the summit of the cataract, atud then gradually brings it round in front of the lens, behind the ciliary processes; which done, it becomes visible through the pupil; the point is now raised again, its flat side is applied to the upper part of the lens, and by a gentle sweep of the needle the cataract is deeply buried in the vitreous humoi". There is another operation with the needle, but it is one commonly contined to cases of congenital cataract. In this instance, the needle is thrust into the transparcut cornea near its junction with the sclerotica, and carried with its flat side opposed in the iris parellel with and close to that membrane, taking especial care not to wound it; here, the ubject is to lacerate and destroy the capsule only, to an extent equal to the circumference of the pupil, and then simply to sink the needle into the body of the lens; or, another method is, to penetrate the sclerotica with the ncedle about une-eighth of an inch from its union with the cornea, push the point almost to the centre of the lens and then turn it forward through the pupil, and afterwards backward again, so as to break both the lens and its capsule into many pieces and drive them before the needle into the aqueous humor: the principle of both these methods being to expose the substance of the chrystalline to the aqueous humor, which is beliered to be a solvent for it, whereby in process of time the cataract becomes dissslved and ahsorbed, and the other parts ultimately recover their original integrity and transparency.

But the most finished operation is extruction. A spear-pointed knife, varied a little in make according to the conceptions of the operator, is thrust through the cornea junt above its transverse diameter and before its union with the sclerotica, and is pushed, with its side parellel with and close to the iris, through the same portion of cornea opposite, until about one-third of the blade is protruded from the eyc; after which it is pressed slowly downward that it may cut its way out below: this makes a section of rather more than one-half of the cornea, and on the neatress and cleanness of this section mainly rests the credit of the operator. This done, a needle, made of nealed gold (to render it the more pliahle) is in- 
not performed on horses. In answer to this inquiry we cannot go the length to say that such an operation is impossible, but we may with truth declare it to be impracticable; and add to this, that, were it practicaable, it is one that, the least reflection would convince these querists, can never prove serviceable to such an animal as a horse. Professor COLEMAN informs us, that, after contending with difficulties that rendered the performance extremely hazardous and the event almost to a certainty ruinous, he did succeed in completing the operation for extraction, and the way in which he proceeded was this-He was compelled first to fix a double tenaculum into the opaque cornea to resist the action of the retractor, which resistance he calculated to be equal to half the force exertible by a man's arm: by this means the globe was kept advanced and tolerably steady, and at the same time the membrana nictitans was retained within the orbit. Should the eye break loose during the operation, it will be so forcibly withdrawn that the vitreous humor will be driven through the section; and the same thing is likely to happen if the globe be set at liberty suddenly or incautiously. But, even granting that the operation has been managed so as to afford some prospect of success, the compression occasioned by the joint-operation of the retractor and membrana nictitans squeezes out the aqueous humor just as fast as it is secreted; or,

troduced through the pupil, and the capsule of the lens is punctured and torn by means of it: in consequence of this, the lens immediately escapes and falls against the pupil, and is afterwards forced out, through the aperture, by a little gentle pressure upon the globe. 


\section{Cataract.}

should union eventually take place, the iris is almost for a certainty implicated in the adhesions : and thus it happens that all our hopes are defeated and all our argument silenced by such a string of unfavorable and disastrous events. The Professor therefore has wisely and humanely relinquished such attempts.

These however are only the difficulties (perhaps not all of them) met with in performing the operation : let us now inquire into the obstacles to its practicability at all. Every man who pretends to a knowledge of optics must be perfectly aware that after the loss of the lens the eye requires that art should in some way or another supply the deficiency, furnish the organ with another refractor, or how can the rays be concentrated upon the retina? Glass is the material used for this purpose, and it is variously fashioned into spectacles and other forms convenient for our use; but I should entertain no hopes myself of the services of a spectacled horse, much less should I be a volunteer to ride him! But, even suppose for a moment that we could contrive a pair of spectacles for him, I should like to know how we are to ascertain what particular lens was adapted to his sight? and which glass suited him for near vision, and which for distant vision? Independently however of all this, there is yet another objection to the operation, one that the practitioner should constantly bear in mind. Cataract in the eyes of horses being, as I stated before, almost invariably a sequel of inflammatory action, and various other parts being at the time likewise the seat of disease, it is rarely unaccompanied with morbid alterations in other textures: in fact, it too often happens that there is a total disorganization of the whole contents of the globe; unless, 
therefore, we can perform miracles as well is operations, and restore all these altered parts to their pristine condition, we had hetter never think of handling a cataract-knife.

\section{Opacities.}

WE meet in practice with two kinds of opacities or films upon the cornea; and it is of importance that we should he able to make a distinction between them, because one is very often removeable, the other never is.

The permanent opacity is the result of that interstitial alteration of texture which is one of the sequelæ of the specific opthalmia. It has a yellow or brown tinge when it first makes its appearance; but afterwards it changes to a diffused muddiness over the whole cornea, thereby completely destroying both its lustre and pellucidity. We must take care however not to confound this with the turbid condition of the cornea resulting from vascular distention and concomitant effusion : this is one of the symptoms of the opthalmia itself-is only present with the inflammatory action; the other is a sequel of it, remaining the same in the interval of remission, and consists in fact in an organic change of the corneal texture.

The other species of opacity has been distinguished by Mr. Travers by the term, nebula; and the cornea has, in fact, a truly clouded appearance. This is a conjunctival affection: its superficiality is evident on a lateral glance of the eye; and it is moreover distinguishable from the other by its remarkably white aspect, and still more certainly (when we can obtain the information) by its origin. The common forerunner of 
the nebula is a blow or bite; though it may result from a violent attack of conjunctival inflammation : in one case it consists of adhesive matter deposited to fill up the breach; in the other it resolves itself into a thickening partial or entire of the conjunctive membrane. It is not uncommon to see the whole cornea obscured in this way in the course of a few hours in consequence of external injury. So long as the adventitious matter remains unaltered or unorganized, so long I apprehend there remains a probability of its being removed; but, when once it has become organized and assimilated with the corneal substance, I should conceive that it must be viewed as a permanent blemish : the elapse of time therefore becomes an important inquiry and consideration in our prognosis.

Should appearances denote in Hammatory action, that ought to be first subdued by proper antiphlogistic means; for it very often happens that with its subsidence disappears the opacity. Should that turn out not to be the case, we may next make use of some escharotic or stimulating application in order to excite increased vascular action afresh, and thereby arouse that of the absorbents. The agent employed for this purpose should fall short of an escharotic; but even caustics may be used provided they are reduced down to this point. There is an endless variety of collyria and eycpowders recommended for these cases. Farriers are very fond of blowing finely powdered glass into the eye, which operates simply as a mechanical irritunt. Calomel, similarly used, has a chemical effect also. I prefer myself an eye-water to an eye-powder : it is applied, I think, both with more facility and effect. Une. 
or the other of the subjoined collyria* will commonly answer our end: it should be injected with a small syringe introduced into the outer canthus. Laxative and diuretic medicine exhibited at intervals will often prove serviceable.

\section{Amaurosis.}

Mork commonly called gutta serena, the glass-eye of farriers, denotes that state of the nervous structures immediately concerned in vision in which they are partly or entirely insensible to light. The characteristic symptoms of this disorder are, a preter-naturally dilated and motionless pupil, which, so far from betraying any visible defect, has an unusually lucid or glassy aspect. Though this is the case commonly in the human subject, yet, in man, there are many notable exceptions; such as the pupil retaining its proper dimensions and being capable of motion, or even becoming actually more contracted than in health : and there are cases on record in which the activity of the pupil has become increased. I know of no such varieties myself among horses: in them amaurosis is simply a state of nervous insensibility, referable in every case that has hitherto come to my knowledge to injury or disease of the brain. The effect can also be at any

* Re Hydrarg. Oxy-Mur. gr.j.

Aquæ Distillat. ₹j. M.

or this,

R Argenti Nitrat. gr. iij.

Aquæ Distillat. 3 j. M.

Fiat collyrium simel in die injiciendum in canthum externum oculi affecti. 
time artificially produced by the application of belladonna to the eye. Professor Colmun relates the case of a horse of his own who received a blow upon the side of the head from falling backward. After the fall, hemiplegia invaded the opposite side of the body, and the corresponding eye became amaurotic. Here, then, the brain, not the eye, was affected. Bleeding, purging, and stimulants to the insensible eye, recovered this horse. The case probably would not have terminated so favorably. had both eyes become paralytic. There are cases of amaurosis, I am told, to be met. with in which there is no discoverable cerebral affection; I do not doubt it, but, for my own part, when there was no other cause apparent I should always direct my curative means principally to the brain; and they should be of a depletory description. But, as this is a disease of rare occurrence, and one about which our knowledge is yet very limited, I shall not waste time in speculation and hypothesis. In conclusion, it falls to my lot to broach an interesting subject which seems either entirely unknown to our veterinarians, or, at least, to this day, has remained unnoticed by them: I mean

\section{Worm in the Eye.}

My own attention has been so recently called to the subject that I have not had time to investigate it, or even give it much consideration; all I profess to do here is, to state some particulars relating thereunto, the perusal of which probably may gratify those who are curious and desirous of any information contributive to our stock of professional knowledge. In a letter, dated June 24th, 1825, from my cousin, who is station- 
ed with his regiment at Meerat, in the northern part of India, I received the following communication.

"In low humid situations in India, where fogs are prevalent or where there is stagnant water, especially after an unusually wet season, worm in the eye is a very common occurrence; it is also seen occasionally in other parts during the cold months, from the begimning of October to the latter end of February, and particularly during the continuance of an easterly wind. And what seems very remarkabie, is, that these horses are frequently affected at the same time with what the Indians call kummiree, which means wealiness in the loins. I have had to treat two cases since I joined the regiment (1824) both of which occurred within the cold season. The first was shewn to me the very day after I arrived by the farrier-major, who reported the horse to have received some injury, and the eye to have been bad for the preceding fortniglit. At this time the lids were shut, the conjunctiva was highly inflamed, the cornea was obscured from common nebulous effusion, and its vessels were filled with red blood. On close inspection I could discern a small white worm floating about in the aqueous humor, at one time rising to the superior, at another sinking to the botton of the chamber. The following morning I determined on giving this little imprisoned intruder its liberty. To this end I punctured the cornea close to its sclerotic margin; which done, the worm escaped with the humor, and was received upon a napkin. It resembles a piece of fine white thread, and belongs to the genus filaria; it measures about one inch and a quarter in length and grows finely pointed at either extremity. Viewed through a microscope it has the appearance of catgut, and five luminous 
marks are distinguishable upon one end of its body which we suppose to be the head; farther backward there is an irregular luminous spot, and from this two brilliant lines run parellel to the other extremity. After the operation I employed simple antiphlogistics, and the horse was sent to his duty six weeks afterwards with an opaque patch upon the cornea which resisted all our ordinary remedies.-The second case unfortunately I did not see, owing to indisposition, until the third day after its reported attack, at which time the eye had the same general aspect as the first; though the serjeant-major informed me that it had appeared dull for some days before, but that the corneal opacity had formed in the course of one night. I treated this case as I did the first. In the first fortnight the upper half of the cornea became clear again, but the remaining nebula did not disappear for two months after that; however, I effected its entire removal in the end, and I think I should have been equally successful in the former case had its origin been of more recent date. If the worm is suffered to remain, the case terminates in blindness."

"I have been favored lately with some observations on the presence of these worms in horses by W. TwINING, Esq. (Surgeon to the Commander in Chief) who has written on the subject in the Medical Society's Transactions at Calcutta, from which I will now give you an extract.-He says Sir E. Home mentions (in his ' Lectures on Comparative Anatomy,') that the arteries of the ciliary processes in the horse are comparatively very large; and that Sir $\mathrm{E}$. is led to believe that the two species of worms, the strongylus and filaria, found alive in the aqueous humor of that animal in India, ob- 
tain access through these arteries; and this opinion is the more probable since similar worms are known to be occasionally present in the blood. Mr. T. also observes, that worms of the same kind have been found in the stomach and intestines of horses by Drs. Giв a and Hartman, Superintendants of the Company's Studs; also, by the latter gentleman, in the cellular membrane, about the loins in particular, in great numbers, and to their presence there Mr. T. ascribes the kummiree: so that what has hitherto appeared to be a strange and unaccountable sympathy between the eyes and the loins, is probably nothing more than the same affection prevalent in the system generally. Mr. T. adds, that several circumstances concur to render it probable that the ova of the filaria equi are received into the stomach with the food, and that these ova, taken up by the absorbents, flow with the blood into various parts of the body favorable to the development of the worm, which having been accomplished, its further progress may be impeded by its augmented size. Sir A. Cooper's observations on worms found within the cœliac artery of the ass may be considered corroboratory of all this; though it must be confessed the subject yet lacks elucidation. Dr. GiB B, who has now had the management of one of the Company's Studs sixteen years, has had on an average twenty of these cases annually. I have got the two worms I extracted preserved in spirits, and look forward to the pleasure of depositing the preparation before many more years have elapsed in my uncle's museum.

"Charles Percivali, "V. S. 11th Light Dragoons." 


\section{LECTURE LXV.}

\section{Dissection of the Brain.}

IN my lecture " on the brain and nervous system*," I defined the brain to be, "that soft white mass which fills the cavity of the skull." I therein observed, that the brain in horses was but small when compared to the bulk of the body; and that it was divided into three portions the cerebrum, cerebellum, and medulla oblongata. I also stated, that it had three coverings or membranes; the dura mater, pia mater, and tunica arachnoides. I then proceeded to speak of the texture and organization of the brain and nerves, and concluded with some general observations on their physiology. It now remains therefore to inquire into the particular anatomical conformation of the brain, and to make ourselves acquainted with the appearances and relative position of its several constituent parts, not only that we may be able to assign particular places of origin to the nerves, but that we may have clearer notions of the seat and nature of cerebral diseasest.

* Lecture x. Part I. which had better now be read over again.

t In order to expose the brain for dissection, after having denuded the anterior and lateral parts of the cranium, first, the 
In raising the bony cap we meet with considerable resistance from the connections between the bone and dura mater, which, I stated formerly, are of a cellular, fibrous, and vascular nature: when these have been torn through, the surface of the membrane shows here and there bloody spots, which are the ruptured mouths of the blood-vessels. In describing the texture and disposition of the dura mater in the lecture referred to, I made mention of certain expansions or processes, and canals or sinuses belonging to it: I shall now proceed to point them out.

Thi plocesses are formed by duplications of the membrane, and are two in number: the falx and the tentorium. The falx or longitudinal process, so named from its being resembled to the blade of a scythe, and indeed the comparison is a striking one, is that expansion of the membrane which descends for a short distance betwen the lobes of the cerebrum. It takes its rise from the crista galli, including that process between its laminæ, from which it curves forward and upward, adhering in its way, first to the frontal, then to the entire length of the sagittal suture, growing broader as it proceeds; at length it terminates upon a thin transverse plate of bone, a process of the os occipitis anterior, where its lamina split, diverge, and disappear in continuity with the tentorium. Whatever may be the use of the falx it seems to be a part whose presence can be dispensed with; for I have a cranium now be-

frontal bones should be sawn through transversely, in a direct line with the summits of the orbital arches; the saw should then be directed longitudinally, on either side, from the extremity of the transverse section, through the temporal muscle, so as to carry the incision ahove the condyloid process of the occiput. 
fore me in which it may be said to be deficient; at least, all such appearance consists in a small triangular piece of membrane which does not project more than an inch from the tentorium: and this cranium belonged to a young horse who was perfectly fiee from any phrenetic disorder. The falx is commonly said however to stay one lobe of the cerebrum from falling or pressing upon the other when the head is inclined to one side.

The tentorium or trunsverse process is extended, like an arch, from the cerebral plate of the os occipitis along the sides of the cranium to its base, whence, greatly diminished in breadth, it may be traced onward to the body of the os sphenoides, where it vanishes in the common covering of the dura mater. It is composed of two lamina: one is continuous with the falx; the other joins that portion of the membrane which covers the cerebellum. The tentorium is equally divided by the falx into two lateral portions. There is some variation to be seen in different subjects in the figure and extent of this process, but I have not remarked that it is ever deficient. It forms a transverse intersection within the cavity of the cranium, or partial septum betweeen the cerebrum and cerebellum, and appears to be of service in fencing these important parts in their proper places, and keeping them, during the various motions and positions of the head, from pressing upon each other.

THE SIN USES most worthy of notice are-The superior or longitudinal sinus, which runs within the duplicature of the falx, along its superior border. It begins in a narrow channel, gradually widens and assumes a triangular figure as it proceeds backward, and terminates with the falx at the cerebral process, where the lateral 
sinuses and it all conjoin their canals. Internally, this sinus presents to view an uneven surface upon which the veins are seen terminating by large open orifices that discharge their blood in the same direction which the stream takes within the sinus itself; here and there also are some slender cords crossing the canal from side to side, which serve to strengthen its parietes and prevent over-distention.

The two lateral sinuses are formed within the duplicature of that part of the tentorium which is attached to the temporal and occipital bones; one extending to the right side, the other to the left side. They begin at the cerebral process, where the longitudinal sinus ends, and they terminate at the foramina lacera basis cranii in the jugular veins. Their canals are also triangular, and are intersected with fibrous filaments. They receive veins both from the cerebrum and cerebellum.

The cavernous simuses, so named from the cavernous appearance of their interior which includes a structure having some resemblance to the corpus cavernosum penis, are formed out of the dura mater upon the body of the os sphenoides. These cavities are remarkable from receiving some important nervous trunks in their passage from the brain, and for lodging the terminations of the internal carotid arteries. They commonly communicate with the

Sub-occipital sinuses. These are also of membranous formation, and are found upon the cuneiform process of the os occipitis, running longitudinally to the foramen magnum. They receive veins from the cerebellum. and posterior parts of the cerebrum.

The dura mater removed, we expose the membrana arachnoides. I have but little to add to the description 
already given of it. It is with difficulty demonstrable from its close and uniform application to the pia mater; we may now and then however succeed in raising it by inflation, and the most favorable places for the experiment are, the base of the medulla oblongata, and the fissures made by the lobes of the cerebellum. From its extreme tenuity and total apparent want of bloodvessels, it is so transparent that the parts underneath appear to be without any such covering : at least, this is its aspect in health; but, in the human subject, in whom these parts are more developed, it is said in a state of inflammation to betray some appearance of vascularity. Between this membrane and the dura mater is exhaled a vaporous secretion.

In regard to the pia mater, I may remark, that the processes detached by it into the substance of the brain are only in contact with the cortical parts; and that the numerous blood-vessels which are seen ramifying upon it, are for the purpose of transmitting others of smaller size into the interior of the cerebral substance, which, though they here appear to pervade only the cortical, are ultimately destined for the supply of the medullary matter. This membrane not only clothes the convoluted exterior of the cerebral mass, passing from one hemisphere to the other, over the surface of their uniting medium, the corpus callosum, but it also gains admission into the ventricles and lines them, and envelopes and confines in their proper places the plexus choroides.

\section{Cerebrum.}

THE largest portion of the cerebral mass, and that which presents itself to our view on raising the skull-

PAR' III. 
cap, is the cerebrum. It is lodged in that capacious elliptical chamber which is formed in front by the parietal bones, behind by the ethmoid, sphenoid, and posterior occipital bones, laterally by the temporal bones, inferiorly by the roof's of the frontal sinuses, and by the ethmoidal plates, and superiorly by the tentorium. The visible surface of it may be said to represent a convex semi-oval, of which the broader end is turned upward. It is equally divided by a longitudinal fissure along its middle, into which the falx cercbri descends; and its divisions, which are perfectly symmetrical, both internally and externally, are denominated hemispheres.

In parting the hemispheres, so as to admit light between them, by which a few small inter-passing vessels and some weak cellular connections are lacerated, we perceive a white, longitudinal body, covered by pia mater, uniting the hemispheres at the bottom of this fissure: this is the corpus callosum. Each hemisphere, separately regarded, has an outward waved or convoluted surface, which is adapted to the undulated concavity of the skull-cap; an inward perpendicular plane, which is opposed to the opposite hemisphere; and a base, divided into lobes, the description of which I shall postpone until the interior parts have been examined.

In the dissection of the brain I shall pursue the customary method of the schools of anatomy, and in describing its several parts preserve the names used in human phrenology so long as I find them applicable to the correspondent appearances. The first step consists in making a clean horizontal section of the brain, parellel to its present convexity, by laying the knife flat upon the surface of the corpus callosum and making a sweeping cut, with its edge first inclined a little up- 
ward then a little downward, through one hemisphere at a time. There being no distinguishable parts in the abscised portions, they may be cast away; but the surface which their removal has exposed being one that presents the greatest superficial extent of medullary substance that can be shewn at one view, the appearance altogether has been described as the centrum ovale*. The form and disposition of the corpus callosum are now demonstrable. It is placed in the centre of this medullary convexity, with which it is continuous in substance; it is narrower below than above; it unites the medulla of the two hemispheres in the middle; inferiorly, it passes between the hemispheres, turns backward and terminates at the base of the brain, in the crura cerebri; superiorly, it joins the fornix, and spreads upon the hippocampi; and anteriorly, it may be said to form the roof of the lateral ventricles. Running along its surface are two prominent lines of medullary matter, between which is a longitudinal linear depression named the raphe.

By making a longitudinal incision through the corpus callosum, on either side of the raphe, we penetrate a cavity in the innermost part of the brain, which, with its fellow within the opposite hemisphere, we distinguish as the lateral ventricles. Having freely opened it, we find a watery fluid within, which serves to keep its parietal surfaces constantly wet, and to prevent adhesion of them. The figure of the ventricle, both from

* The cortical matter however cannot be all pared away-it will intrude in places and interrupt the uniformity of the surface: indeed, the horse's brain will less admit of being carved into such an aspect in consequence of the proportionably grcaler quantity of cortical substance in its composition. 
its construction and from the inequalities of its contents, is extremely irregular. It extends as far forward as the firont incurvation of the corpus callosim, from whence a winding canal is continued forward and downward to the base of the anterior lobe of the cerebrum, where it ends in a small circular conduit thatleads into the ethmoidal ventricle: this canal, from its being represented to wind in its course like a horn, has got the name of the inferior cornu. From the superior part of the cavity, another flexuous passage, smaller than the former one, taking a direction outward, then downward, and lastly forward, leads to the base of the brain, through the substance of the middle lobe, and there has a blind termination; from which circumstance, and from its cylindroid figure, some call it the digital cavity, others the superior cornu. The ventricles are lined (and the parts within them covered) by an extension of pia mater, which finds its way into them along with the vessels forming the choroid plexus. By this membrane, whose vascularity appears to be lower than that of the pia mater elsewhere, the watery fluid, it is supposed, is secreted: unlike serous cavities in general however, the ventricles appear to hold water during life; for, if an animal is put to death suddenly, and these cavities are opened with all possible expedition, water, and not vapor, is uniformly found in them.

We now come to what are considered as the contents of the lateral ventricles. They are-the corpora striata, the hippocampi, the plexus choroides, the fornix, and the thalami nervorum opticorum. But, a view of all these parts cannot be obtained until we have reflected the corpus callosum; prior to which being done, that body should be cautiously raised in order to show the 
Septum lucidum, the translucent medullary partition between the ventricles, which extends perpendicularly along the corpus callosum in front, and is attached to the fornix behind. It is broad inferiorly, grows gradually narrow superiorly, where it ends in a point, at the angular junction of the corpus callosum with the fornix. It consists of two thin lamine of medullary matter, included between and supported by the membranous linings of the ventricles, in the middle of which is a fissure or small cavity, by some described as the fifth ventricle, but generally known as the fossa Sylvii. Sometimes this fissure is very demonstrable and glistens interiorly with moisture; at other times it is hardly perceptible.

When the corpus callosum is turned back, four large and remarkable eminences present themselves, two upon each side of the septum lucidum. The two inferior, and most bulky, are the corpora striata. They rise out of the lower and back parts of the ventricles, project. into the middle of those cavities, where they become broad and approach the septum, growing narrow and receding from each other above, below extending along the anterior cornua. Externally, they have a thin lamina of medullary matter, but this is somewhat obscured by the cortical substance of which their interior is exclusively composed.

The two superior eminences, smaller and whiter than the last, whase anterior extremities lie between the posterior of the corpora striata, are the hippocampi. These bodies, remarkable for their prominence and whiteness, occupy the superior spaces of the ventricles, where they lie in contact with the septum; but, in proceeding backward, they diverge, stretch first outward, then 
downward, and lastly forward; in fact, they descend into the superior cornua, and there end in bulbous extremities, called the pedes hippocampi. This descent or elongation, which may be denominated the crus hippocampi, is continuous above with the crus fornicis, but below it gets to the outward part of the crus fornicis, and gives off a thin, plaited, medullary border, which may be said to be an extension of the corpus fimbriatum. Their medullary covering appears to be continued from the corpus callosum; their internal cortical substance, which is interspersed with medullary strix, originates from the very middle of the hemispheres. If sliced, they will be found to consist of alternate laminæ of medullary and cortical matter.

Deeply lodged in the channel between the corpus striatum and hippocampus, lies a red, soft, vascular substance, consisting of a plexus or collection of minute blood-vessels, invested in an extension of pia mater, called the plexus choroides. This plexus first makes its appearance from behind the fornix; inferiorly, it ends abruptly in a round bulbous mass; superiorly, it sends down a process into the superior cornu. The arteries composing it come from the posterior arteries of the cerebrum: they enter the interior of the brain along a fissure, which in one place is a complete canal, existing between the posterior lobes of the cerebrum, and gain admission into the ventricles around the sides of - the fornix. Its veins assemble and form a large branch, the vena Galeni, which branch unites with a similar one coming from the opposite plexus; the two conjoined make a single trunk, and that proceeds upward, along the above mentioned fissure.

The fornix is that part which receives the posterior 
border of the septum lucidum. It is extended, after the manner of an arch, between the corpora striata below and the heads of the hippocampi above, where it forms a junction with the corpus callosum, which it meets at an acute angle. It has four processes or crura. The two inferior crura spring from the corpus albicantium, at the base of the brain; in their course forward from which, they approximate and unite into one main crus or pillar; thus united, they wind upward, describing an arc, and, as soon as they make their appearance within the ventricles, constitute the body of the fornix. The superior crura, which are comparatively slender, proceed from the upper end of the fornix, wind upward and then descend into the superior cornua of the lateral ventricles, where they grow tapering and at length end in sharp-pointed extremities. Their anterior or concave edges, which are thin, unattached, and somewhat uneven, have been named the corpora fimbriata ; their posterior borders are continuous with the crura hippocampi : along their fimbriated edges run the lateral processes of the plexus choroides. The middle part or arch of the fornix is unattached; but its superior or broad part is contiguously applied behind to the thalami, and adheres to them through the intervention of a fine membrane, investing the vessels of the choroid plexus which are ramifying hereabouts, named the velum interpositum: from the circumstance of the back of the fornix being imprinted by these vessels with many minute linear grooves, mostly running obliquely, this surface of it is commonly described as the lyra, psalterium, or harp.

Having divided and reflected the fornix, and turned back the heads of the hippocanıi, we bring into view 
the thalami nervorum opticorum. These bodies are also said to be in the lateral ventricles: more properly speaking, they form the upper and back parts of those cavities. They are white, conoid in form, narrow and approximated inferiorly, where they lie between, and rather behind, the corpora striata; broad and directed opposite ways superiorly; in front they are opposed to the fornix, which they in a measure support; and behind they contract into medullary bands-the tractus optici, which turn round the crura cerebri to the base of the brain. Upon these bodies the separate portions of the plexus choroides unite into a single vascular cord, which takes its course in the canal between them, and makes its exit from the brain in the fissure left between the posterior lobes of the cerebrum. The thalamiare firmer in their composition than the corpora striata; like those bodies however they are cortical within, thinly striated with medullary matter.

In the groove between the thalamus and corpus striatum, partly covered by the plexus choroides, runs a conspicuously white, medullary band, designated the centrum semicirculare geminum, vel tania semicircularis.

The contiguous parts of the thalami, flattened and closely applied, are united in one broad circular place by pulpy cortical matter, which union is called their commissura mollis.

Immediately behind the fore part of the fornix, runs transversely a firm medullary cord, which is extended in a curved direction, on either side, into the substance of the corpus striatum : this forms a band of connection between the hemispheres, and takes the name of commissura inferior cerebri.

Superiorly, above the commissura mollis, is another 
medullary cord, similar to the one last mentioned, but shorter and smaller, which is the commissura superior cerebri.

Just over the commissura inferior, between it and the commissura mollis, between and in front of the optic thalami, under the arch of the fornix, is a triangular hole, called the foramen commune inferius. Now, as the lateral ventricles communicate under the arch of the fornix it is evident that both of them must open into this foramen; and as this foramen leads into the third ventricle, it follows that this cavity and the lateral sinuses must reciprocally communicate by means of it.

Between the commissura mollis and the commissura superior is another foramen, which would also be one of like communication were it not covered and closed by the velum interpositum, which is here spread over the surfaces of the thalami, and traversed by the vessels of the choroid plexuses.

The third ventricle, as it is called, is nothing more than the fissure which exists between the thalami, in consequence of their partial divergence, below and behind the commissura mollis, in the medium line of the brain. The floor or back part of this narrow oblong cavity, is constituted of the crura cerebri.

Leading from the inferior part of this ventricle is the infundibulum, a passage that is small at its commencement, but gradually enlarges to its termination, which is in a blind extremity, in front of the corpus albicantium, at the pituatury gland. Superiorly, under the superior commissure, is a passage leading from this cavity into the fourth ventricle, the iter a tertio ad quartum ventriculum, which is situated in the cerebellum. 
Over the third ventricle, deeply lodged between the summits of the thalami, above and rather before the superior commissure, we find the pineal gland-a little, conoid, greyish body, marked by a slight depression along its middle, enveloped in pia mater, which it derives from the vessels of the choroid plexus, and attached by that membrane to the thalami, and the tubercula quadrigemina, which are placed behind it. From its base or inferior part proceed two little peduncles or foot-stalks, which run along the top of the superior commissure, and are implanted into the crura cerebri. The structure of this little organ remains unknown. It is possessed of considerable firmness; it is deeply reddened and highly vascular within; but I have not remarked that it contains earthy matter, as is the case so frequently with that of the human brain.

Above the third ventricle, behind the pineal gland, and immediately over the iter a tertio ad quartum ventriculum, are four considerable eminences, the tubercula quadrigemina - or rather bigemina, since they are divided into the rwo nates and Two testes. The nutes, the foremost and by much the largest of these bodies, separated by a groove from the testes, and by a deep perpendicular fissure from each other, present semi-oval exteriors, of a mixed composition, cineritious and medullary, and are in intimate union with each other and with the testes. The testes, much smaller than the nates, are also ovoid in figure, but their long diameters are placed contrariwise-transversely. In their composition they are alike also, as well as in their connections, both being joined to the crura cerebri, by which they are supported. 


\section{Cerebellum.}

Is order to guard against a common misconception of the relative position of this part, I shall repeat here what I took occasion to lay some stress upon in a former lecture*-that the three divisions of the horse's brain are similarly lodged, in regard to their cranial case, to what the correspondent parts in man are, but that when we view them as the animals naturally stand, their relative situation is altogether altered. The cerebellum (as in man) occupies that compartment in the basis of the cranium which is formed above and behind by the anterior occipital bones, laterally by the petrous portions of the temporal bones, and superiorly and anteriorly by the tentorium; that septum being deficient inferiorly and anteriorly to give passage to the crura cerebri to form a junction with the cerebellum, while the vacuity admits also of the projection of the anterior vermiform process, which is the only portion of the latter that does not rest upon the tentorium.

In volume, figure, and aspect, the cerebellum is at once distinguishable from the cerebrum. It is only one-sixth of the size of the cerebrum. Its figure is irregular-it has two oval ends, placed transversely, united in the middle by a broad and prominent vermiform belt: its lateral dimension exceeds its longitudinal. Its aspect is lobular or convoluted; but it is everywhere so striated with deep transverse fissures that its appearance is different from any other part of the organ.

The cerebellum is superficially divided into three oblong lobes:-a middle loles, and two lateral lobe. The 
lateral lobes, which are opposed to the posterior lobes of the cerebrum, are small and ovoid; the middle lobe is prominent, arched, and subdivided into lobuli by several sulci, conspicuous for their depth, crossing its upper and under surfaces. The anterior and more prominent part of this lobe, which is received into the great fissure of the cerebrum, is denominated the anterior vermiform process; the continuation of it, along the summit of the head,forms the posterior vermiform process.

In the cerebellum, the disposition and proportionate quantities of medullary and cortical matters, are reversed, with regard to the cerebrum. Here the cortical substance exceeds the medullary in quantity, and, instead of forming the outward parts, pervades the innermost. Slice the cerebellum horizontally, and these substances present a laminated appearance; make a vertical section of it, and they are so disposed as to give the surface an arborescent representation, usually denominated the arbor vita.

Within the cerebellum, or rather between it and the tuber annulare and medulla oblongata, which last form its back part, is situated the fourth ventricle. Beginning above the testes, where it is perforated by the iter a tertio, it extends upward and backward to the extremity of the medulla oblongata, where it ends in a sharp angular point, which, with a groove running from it along the floor of the ventricle, anatomists have resembled to a pen, and thence called the calamus scriptorius.

Extended across the inferior and anterior part of the ventricle, closing the cavity between the testes and lower extremity of the anterior vermiform process, is a mem-

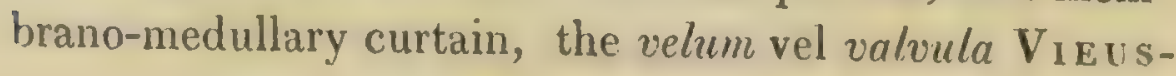


SENII. It is supported at the sides by two medullary productions, remarkable for their whiteness, the processus a cerebello ad testes, which some regard as the columns of the valve.

The choroid plexus of the cerebellum is found, lying crosswise, in the posterior part of the fourth ventricle, between the cerebellum and medulla oblongata. It nowise differs in its composition from the plexuses of the lateral ventricles. It is distributed into three principal divisions: one lies in the middle of the calamus; the other two, lateral, are lodged in fissures in the cerebellum, occupying the spaces between it and the tuber annulare.

\section{Posterior Surface of the Brain.}

THE base or posterior part of the cerebrum is divided into six lobes: two inferior or anterior, and two middle, which rest upon the alæ of the ethmoid bone, and two superior or posterior, which are lodged in the fossæ of the squamous portions of the temporal bones.

Upon the middle lobes are two broad prominences whose surfaces are remarkable from being perfectly smooth: these are the bases of the corpora striata, the parts from which the olfactory nerves are seen taking their origin.

Above these, in the middle of the brain, are two oblong medullary eminences intimately united together: these are the crura cerebri. They rest upon the alæ of the sphenoid bone. They take their rise from the inferior and middle lobes of the cerebrum, and are continued into an ovoid protuberance above them, named the tuber annulare. Though medullary without, the 
crus, when cut deeply into, exhibits a cineritious hue, and the part that is the most darkly shaded has been called the locus niger.

Seated between the crura is a small, hemispherical, medullary eminence, distinguished as the corpus albicantium. In the human subject this body is double; and there is some appearance of a depression along its middle in the horse. It is medullary without, but has a tinge of cortical matter within.

Winding obliquely downward, around the crura, proceeding from the terminations of the thalami, are the tractus optici: they are to be regarded as the roots of the optic nerves, with which they are continuous. They are entirely medullary.

Higher up and more outward than the crura cerebri, are the crura cerebelli-two stout, cylindroid, medullary cords joining the lateral lobes of the cerebellum to the tuber annulare. These parts consist of a prolongation of the medullary substance which forms the trunks of the arbor vitæ.

The tuber annulare vel pons VAROLII, whose situatiun now I need not point out, may be said to be constituted of the united substances of the crura cerebri and crura cerebelli : it is, in fact, the common termination or medium of junction of the cerebrum and cerebellum. It is supported by the cuneiform process of the posterior occipital bone. Its surface is marked by a shallow depression along its middle, which gives it the appearance of having lateral eminences. Internally, the tuber exhibits a mixture of cortical and medullary matter.

Above and below the tuber, centrically situated, are little round depressions or blind holes, which are ge- 
nerally described as the foramina caca, superior et inferior. They serve as places of lodgment for small plexuses of blood-vessels.

Still higher than the tuber is placed the

\section{Medulla Oblongata.}

THE smallest division of the cerebral mass. It rests upon the cuneiform process of the occipital bone, and is continued upward and backward to the foramen magnum. Regarded in an excised state, it is of an oblong, quadrilateral figure. It has a deep fissure along its middle (in which runs the basilar artery) that divides it into lateral portions. Contiguous to this fissure, rising from the sides of it, are two longitudinal eminences, called the Eminentic pyramidales. From the lateral parts project two other eminences, named the Eminentice Olivares. The upper surface of the medulla oblongata forms, with the tuber annulare, the floor of the fourth ventricle. Though, like the tuber annulare, it is composed internally of a mixture of the cortical and medullary substances, its shade is lighter than that body.

Lastly, remains to be considered the

\section{Pituatury Gland.}

A REDDISH body, of an ovoid form, seated upon the sella tursica, within a fold of the dura mater. It has a membranous capsule, surrounded by cellular adhesions, by which it is firmly retained in its place, whence it is not dislodged even though the cerebral mass be taken out. It has the appearance of being highly vascular; it is however of a lighter color internally than externally, and puts on a mottled aspect in places.-The ancients entertained a notion that it received a fluid from the 
brain, which they called pituita, through the infundibulum, and transmitted it to the nose; but we find that these parts have no conmunication whatever : in short, its real use is totally unknown.

\section{Medulla Spinalis.}

Tн е spinal marrow is that extended cylinder of brainlike substance which is continued from the posterior part of the medulla oblongata through the entire length of the spinal canal. Lodged within a cavity whose form is continually undergoing more or less variation, it neither completely fills nor precisely fits that cavity : in somè places it swells in size, in others it grows contracted; everywhere it has the appearance of having shrunk from, or of being disproportionably small for, the canal in which it is contained.

It is inclosed in the same membranes that envelop the brain; but, in addition to them, the superior ligament of the spine serves as a covering and defence to it below. To this, and to the periosteum lining the canal, its proper theca is loosely attached by cellular, adipose, and gelatinous matter. Its dura mater is derived from that which covers the brain : in being continued through the foramen magnum the membrane is contracted into a cylindrical sheath which loosely incases the marrow, and is generally described under the denomination of theca vertebralis. About the beginning of the sacrum, the theca narrows; it recedes from the sides of the canal, and, having inclosed the termination of the marrow, is extended into a point and lost upon the cauda equina. The dura mater is connected to the membranes underneath, the membrana arachnoides and pia mater, by a fine cellular tissue; and these coverings have the same relation to the marrow that the same membranes have to 
the brain, of which they may be considered prolongations.

The spinal marrow, stripped of its membranes, is cylindroid in form, flattened along its upper and under surfaces, each of which is marked by a longitudinal linear fissure: these fissures, of which the inferior is the more conspicuous one, are continuations of those that traverse the medulla oblongata, they equally divide the marrow into two cords, and serve as convenient tracts for the spinal blood-vessels. In places, also, some transverse fissures or little wrinkles are apparent, which are evidently for the purpose of admitting of certain degrees of elongation and contraction, in order to guard against extension or laceration of the marrow under any motion or altered position of the spine. The marrow varies in its dimensions at different parts; and also somewhat in its form. At its beginning, from the medulla oblongata, it is large, from which to the fifth cervical vertebra it gradually grows less in circumference; here it swells again, but again diminishes in entering the first dorsal vertebra; along the back it is small and nearer approaches the form of a perfect cylinder; but in the loins it grows flatter than ever, and, a third time, increases in size, until it reaches the last lumbar vertebra, from which it begins to taper, and continues so to do until it at length ends in a pyramidal point within the second piece of the sacrum. Around its termination come off several large nervous cords, which are continued along the canal, and extend, some of them, into the coccyx: altogether they have been resembled to a horse's tail, and thence denominated canda equina.

In the year $1808 \mathrm{Mr}$. SEWELL conveyed in a letter PART III. 
to Sir E. Ho.nE, which was afterwards read to the Royal Society, the particulars of what he conceived to be a canal running from the fourth ventricle through the whole length of the spinal marrow, containing a limpid colorless fluid, and lined by the membrana arachnoides or a membrane very like it; but, since this, M. BELLENGERI, of the College of Medicine of Turin, has proved, that this supposed canal is in truth a median furrow dividing the spinal marrow into six bands; he has also shown that the roots of the spinal nerves, both anterior and posterior, are triple. This discovery has opened an entirely new field of physiology, and is leading rapidly to very important results affecting patho$\log \mathrm{y}$.

The nerves given off by the spinal marrow are twenty-nine pairs. They originate, the same from either side, by superior and inferior fasciculi or bundles of filaments, which pierce the theca vertebralis in several places, from which they derive cellular sheaths in their egress. The cervical and dorsal nerves, for the most part, arise from the marrow opposite to the foramina through which they make their exit from the spine; but the posterior lumbar and sacral nerves proceed obliquely backward before they turn out; and some of the latter, and all the coccygeal nerves, run longitudinally down the canal before they quit it. The filaments composing the fasciculi are themselves small; they bear a proportion in size and number to the nerves of which they are the roots. Threads of communication may be seen, oftenest in the neck, running from one set of fasciculi to another. The superior fasciculi are separated from those which arise below, by a strong, white, fibrous band which runs along the side 
of the marrow, and has a puckered attachment in one particular place to the theca, between every pair of nerves, on either side; inwardly, it is intimately joined to the pia mater: this band, which is denominated from its several pointed connections the ligamentum denticulatum, prevents that injurious extension of the nerves, when the spine is forcibly flexed, which might occasion laceration of their medulla. In the space between the vertebræ, the superior fasciculi converge and form a ganglion, from the opposite side of which many filaments arise and proceed with the inferior fasciculi and join them, and thus form the trunk of the nerve. 


\section{LECTURE LXVI.}

\section{Origin and Distribution of the Nerves.}

I.T has been observed, in speaking of the nervous system in a former lecture, that there are in all forty-six pairs of nerves: ten coming from the brain, are distinguished as the cerebral nerves; thirty-six from the spinal marrow, denominated the spinal nerves. The nerves, being symmetrical in number and distribution on either side of the body, take their origins by pairs, and these pairs are numbered, and so distinguished from one another, according to the order in which they arise, proceeding from below upward within the head, from before backward within the spine. In addition to this distinction of ordinal number however, every pair of cerebral nerves has obtained a particular epithet significant of the specific purpose they serve, the part they supply, or else some peculiarity in their division or distribution. With these remarks, I shall commence with the origin and distribution of the

CF, EBRAL NERVES.

First Pair or Olfactory Nerves

ARISE from the corpora striata, along the posterior 
borders of which bodies the medullary bands or roots of them may be traced as high up as the middle lobes of the cerebrum. These are the largest of the cerebral nerves, are bulbous at their origin, pulpy in texture, and exhibit, when cut into, comparatively to their size, large cavities, which are walled in by a layer of medullary matter, inclosed within a thinner one of cortical substance. These cavities, which some erroneously describe as ventricles of the brain, since they evidently belong exclusively to these nerves, are conical in figure: from being capacious inferiorly, and generally distended with fluid, they contract upward into small circular canals, which lead through the trunks of the nerves into the anterior cornua of the lateral ventricles. GirARD calls them the ethmoidal sinuses; but, as this would create confusion in our nomenclature, it would be better to name them the olfactory sinuses. This, the tubular portion of the nerve, is sheathed in dura mater. From its abrupt or truncated termination against the cribriform plate of the ethmoid bone, are transmitted numerous, soft, nervous filaments, which pierce the foramina of this bony plate, and enter the nose, where they undergo a further subdivision, and are at length widely dispersed over the schneiderian membrane.

\section{Second Pair or Optic Nerves.}

If we may be allowed to estimate the importance of nerves by their magnitude, the optic will hold the third rank among those of the brain, being excecded in size only by the 1 st and 5 th pairs. They take their roots from the posterior, contracted, and incurvated parts of the thalami nervorum opticorum. At first. 
they wind forward and downward, around the crura cerebri, which radical parts of them have obtained the name of tractus optici; in doing this they approach each other, (in which relative course they are singular, all other pairs diverging) and at length form a junction -an intermixture, some say a decussation, of their fibres, (in which they are also singular) just below the corpus albicantium. Then, they become again two separate nerves, proceed downward and forward through the foramina optica, and enter the cavities of the orbits. Here each nerve continues this oblique course, surrounded by the muscles of the eye, and penetrates the inner, inferior, and posterior part of the eyeball, within the interior of which it expands and forms the retina. In its whole course, it is inclosed within a sheath prolonged from the dura mater, forming one more peculiarity in its distribution.

\section{Third Pair or Motores Oculorum}

$\mathrm{T}_{\mathrm{AKE}}$ their origin, by several filaments, from the inward parts of the crura cerebri, about their middle. The trunk of the nerve, thus formed, first runs obliquely outward, across the back of the crus, then turns downward and enters the cavernous sinus, wherein it is covered by a sheath of dura mater, and continues its course through the foramen lacerum orbitale, inwardly placed to the other nerves, into the orbit. In entering the cavity, the nerve divides into two branches. The smaller is generally received by a single muscle-the levator oculi. The larger branch subdivides into several others : the longest of these runs round the eye-ball and penetrates the obliquus inferior; the two or three others run to the adductor and depressor imuscles. 
Upon the outward side of the optic nerva, between it and that part of the motor oculi from which the branch nerves spring, is situated the OPTHAMIC: GANG IION. This little body is principally constituted of branches from the third pair; but it also receives a filament or two from the sixth. The nervous threads transmitted by the ganglion surround the sheath of the optic nerve, and pursue their course over it, penetrate the globe of the eve, and run to be dispersed upon the iris.

\section{Fourth Pair or Pathetic.}

Thess, the slenderest of the corebral nerves, take a filamentous origin from the summits of the testes, whence they proceed outward between those bodies and the crura cerebelli, creep round the crura cerebri, and are seen at the border of the tentorium entering the cavernous sinus. Having coursed the anterior boundary of the sinus encased in dura mater, it takes refuge again in a small bony canal, constructed for its passage, in front and rather to the outward side of the foramen lacerum, which conducts it into the orbit. Its destination is the superior oblique muscle of the eye, to which it exclusively distributes its ramifications.

\section{Fifth Pair or Par Trigeminum.}

TнesE are the largest nerves of the brain. They take their beginning by a multitude of filaments from the crura cerebelli, and forthwith run for security into the cavemous sinuses, where they are covered and protected by distinct sheaths of dura mater. Each nerve within the sinus suddenly swells in bulk, and thus is said to form a ganglion: and certainly the enlargement is darker-colored than the nerve itself, hut, it does not 
put on the common appearance of, nor is it of the same texture as, those little knots or swellings in other parts which we are in the habit of calling ganglia. Waving this remark however, and considering it as a ganglion, we say that three nerves depart from it; and these we denominate, from the parts which they are principally destined to supply, the opthalmic, the anterior maxillary, and the posterior muxillary nerve.

THE OPTHALMIC NERVE, the smallest of the three divisions, proceeds for a short way down the sinus in union with the superior maxillary; inclining forward however it soon leaves that nerve, and takes its passage through the foramen lacerum orbitale into the orbit. As it emerges from this opening it splits into three branches:- the lachrymul, the supra-orbitar, and the lateral nasal branch.

The lachrymal branch, at its origin, comprehends two divisions. One is a long single nerve that ascends behind the muscles of the eye, through the fatty matter at the bottom of the orbit, winds over the angle formed between the zygoma and the frontal orbital process, and ends in subcutaneous and anastomosing branches about the forehead. The other consists of several filaments which run forward over the fatty matter enveloping the cye, and are distributed to the lachrymal gland, conjunctiva, and ciliary glands of the upper eyelid.

The supra-orbitar branch takes the same superficial course across the roof of the orbit to the inner angle of the orbitar ridge, where it passes through the foramen supra-orbitarium, and afterwards ramifies upon the skin covering the forehead. It gives off a twig or two to the adipose matter of the eye, and sends a branch to the upper lid. 
The lateral nasal branch, the largest of the three, makes a sudden turn inward, and runs between the levator and retractor oculi, and after having detached a considerable branch to the membrana nictitans, it enters again the cavity of the cranium through the foramen orbitale internum, from which it takes its passage, by one of the holes in the cribriform plate, into the chamber of the nose. Herein, it creeps along the top of the anterior turbinated bone within a bony and membranous canal, immediately covered by the more prominent part of the os nasi, and sends its ultimate branches to the false nostril and wing of the nose. Near its origin, this nerve sends a fine filament or two to the opthalmic ganglion.

THE sLCond DIriston or anlerior maxillary nerve, much larger than the opthalmic, leaves the cranium through the foramen rotundum of the sphenoid bone, and takes its passage through the canalis infra-orbitarius, whence it emerges, covered by the levator labii superioris, upon the face: here it splits into several large branches, denominated the facial nerves; or, altogether, from their being imagined to sprout from the trunk after the manner of the claws of the foot of a goose, the pes anserinus. But, prior to its entering this canal, it detaches several branches of importance. 1. A branch which pursues the same course, along the floor of the orbit, to the inner canthus, where it sends filaments to the under eye-lid and lachrynal duct, and disperses its remaining ramifications upon the contiguous skin. 2. Several long filaments which descend upon the tuberosity of the anterior inaxilla, penetrate the bone, and furnish twigs to the antrum and the two superior molar teeth. 3. The largest branch is the spheno- 
palatine or lateral nasal nerve, to which the foramen spheno-palatinum gives passage into the nose, wherein it divides into two sets of filaments. One of these fasciculi is spread over the lateral parietes of the nasal cavity; the other ramifies over the sinuses, and transmits a filament of considerable length along the posterior border of the septum which passes through the foramen incisivum superius to the roof of the mouth. 4. A slender branch which goes to the velum palati. 5. The palato-maxillary nerve is a considerable branch which traverses the canal of that name in company with the palatine blood-vessels, and disperses the majority of its ramifications over the soft palate; though some of its longer ones may be followed through the foramen incisivum inferius into the upper lip.

The terminating or facial branches of this division, which remain to be described, in general comprehend, at their exit from the foramen infra-orbitarium, two large and two small nerves. The principal branch descends in a straight line upon the side of the face, and, about midway between the foramen and front of the lip, shoots forth into many smaller branches, which are dispersed over the side and surrounding border of the upper lip. These nerves receive communicating filaments from the anterior facial branch of the portio dura, and with them form an intricate and important plexus upon the fore and lateral parts of the face. The branch next in size to this takes a similar course, anteriorly to the last, and distributes its ramifications to the nares and muscles clothing them externally. It detaches one long filament which turns round the smooth crescentic border of the anterior maxillary bone, and goes to the muscles internally situated. One of the small 
branches, which takes its course anteriorly to all the others, runs directly to the levator labii superioris. The smallest branch of the four is one of communication with the portio dura.

THE THIRD DIVISION, the posterior maxillary nerve, the largest of the three, leaves the cranium through the lowermost nook in the foramen lacerum basis cranii, and runs forward, defended by the branch of the posterior jaw, across the middle of the pterygoideus, and enters the foramen maxillare anterius. Its branches are- 1 . A reflected branch which runs up in front of the parotid gland and joins the portio dura as the latter turns round the border of the masseter. 2. The buccal nerve, which pierces the superior portion of the pterygoideus, crosses behind the tuberosity of the superior maxilla, penetrates the buccinator, and buries itself in the substance of the cheek, through which it may be traced into the lower lip. 3. Twigs to the pterygoideus. 4 . The gustatory nerve, a branch nearly equal in size to the trunk itself. This, which by itself may be considered as a trunk, and one of importance too, descends by the side of the tongue, penetrates that organ about its middle, and vanishes in its tip. $a$. Its first branch is a long one that takes the same direction as the main (maxillary) trunk, and enters a medullary foramen in the branch of the jaw. $b$. The gustatory then gives off the dental, a long slender nerve which courses the side of the posterior maxilla, concealed by the submaxillary gland to which it sends twigs, as far as the symphysis, where it enters a small foramen and sends its ultimate ramifications to the roots of the incisive teeth. $C$ Many twigs which are detached laterally by the nerve as it courses the substance of the tongue: they take an 
oblique direction, and are destined for the supply of the gustatory papillæ.

The posterior maxillary nerve itself, having entered the interior of the bone, takes a longitudinal course close to the roots of the teeth, into the fangs of which it detaches branches as it passes them, while others ramify in the diploe. Through the foramen maxillare anterius, the remaining portion of this nerve emerges from its bony conduit, and then splits into two branches; of which the anterior subdivides into several smaller ones which ramify upon the integument of the under lip; the posterior runs for some way without giving off a single twig, it then ends in four or five long filaments which expend their ramifications in the interior of the lip.

\section{Sirth Pair or Abducentes}

Arise, by means of filaments, from the medulla oblongata, by the side of the fissure along its middle, near to its junction with the tuber anmulare. This nerve penetrates the cavernous sinus behind the fifth pair, and therein, running between them and the carotid artery, it meets with the opthalmic nerve and accompanies it through the foramen lacerum into the orbit. Here, it gives off two or three filaments to the retractor oculi; but its principal destination is to the abductor, among the fasciculi of which its ramifications are equally distributed.

\section{Seventh Pair or Auditory Nerves.}

THIs pair includes two separate nerves on either side : one, fron its remarkable softness, is denominated the portio mollis; the other, in contra-distiuction, the portio dura. 
'T'нЕ poltro morus takes its rise from the Hoor of the fourth ventricle, by the side of the crena of the calamus scriptorius. It turns round the medulla oblongata, and then passes directly outward and enters the foramen auditorium internum. Having gained admission into the vestibule of the internal ear, it resolves itself into divers pulpy filaments which pierce a perforated bony plate, and ramify over the various parts of the labyrinth

THE PORTIO DCRA arises from the place of union of the medulla oblongata, tuber annulare, and crus cerebelli; and takes its passage through the foramen auditorium internum, inclosed in the same sheath of dura mater with the portiom ollis. At the bottom of the meatus auditorius internus, it detaches two slender but important branches. 1. The chorda tympani, a long slender filament which proceeds into the cavity of the tympanum, crosses therein the neck of the malleus, makes its exit again by a small foramen, and, descending to the root of the tongue, forms a remarkable union with the lingual nerve. 2. The other also runs in the vicinity of the tympanum, but then it pursues afterwards the course of the Eustachian tube. Deeply seated under the os temporis within the fauces at its exit, its branches become distributed over the parts in the immediate vicinity.

The portio dura then departs from the internal ear, issuing from the bone by the spiral canal, deeply buried under the parotid gland, and gives off the auricular nerves, of which there are three principal divisions. 1. The anterior auricular nerve ascends over the fleshy root of the anterior part of the ear, where it detaches some branches which unite with others coming from 
the lachrymal branch of the first division of the fifth pair and form a sort of plexus-the anterior auricular plexus. Its remaining branches are dispersed upon the skin thereabouts. 2. The posterior auricular nerve, less in size, mounts the back of the ear, in company with the blood-vessels, distributing branches to the muscle about its root, and ending in vanishing ramifications upon the skin of the ear. 3. The internal auricular, the least considerable of the three, enters the concha for the supply of the inward parts of the external ear. 4. Purotideal branches, two, three, or four in number, ramifying within the substance of the gland, and transmitting other smaller filaments to the covering skin. Against the articulation of the posterior jaw, the portio dura is joined by a considerable branch from the posterior maxillary nerve. Augmented in size by this union, the portio dura emerges from the substance of the gland, and turns round the cervix of the jaw along the outward side of the temporal artery. Having mounted upon the masseter it divides into two principal branches. The posterior one obliquely crosses the muscle, a little anteriorly to its middle, enveloped in cellular membrane, detaching many filaments in its passage to the fibres of the muscle, but more and larger ones to the skin, and some few of communication with the anterior branch, and then ramifies subcutaneously upon the muscles of the lower lip, and upon the side of the lower jaw. The anterior branch descends over the fore part of the masseter, a little posteriorly to the zygoma, likewise clothed in cellular substance, furnishing in its course muscular, cutaneous, and communicating filaments; having left the masseter, it inclines forward, undiminished in size from the ramifications it 
hils already produced, and shortly after ends in an arborescent expansion over the inferior and anterior part of the face. It sends some filaments to the nuscles thereabouts, and to the upper lip; but its principal ramifications go to join those of the auterior maxillary nerve.

\section{Eighth Pair or Par Vagum.}

THIs pair is constituted of two nerves on either side, so that it may be said to be double:-the accessory or additional one is denominated the glosso-pharyngeus, the other is the proper par vagum. They arise by many filaments from the corpora olivaria, and make their exit through the foramina lacera basis cranii, in company with the nerves next to be described. Having left the skull, we find them lodged, secure from injury, with the nerve of the ninth pair, in a hollow space to the inward side of the condyloid process of the occiput.

The glosso-pharyngeus, by much the smaller of the two, now quits the par vagum, and, turning round the internal carotid artery, runs forward and downward upon the side of the pharynx, insinuating itself among the constrictor fibres, whence it proceeds to the root of the tongue and there disappears. Its branches are1. At its origin, a reflected branch which joins the portio dura just before that nerve pierces the parotid gland. 2. Two branches to the constrictors of the pharynx. 3. Before it reaches the tongue, the nerve splits into three or four branches which ramify and vanish in the base or root of that organ.

The proper par vagum, having disunited froin the glosso-pharyngeal nerve, proceeds downward and backward to join the carotid artery, in the same cellular 
sheath with which, to the outward side of the vessel, it takes its course along the neck to the chest. Its cervical branches are-1. Two or three fine filaments to the superior cervical ganglion. 2. The pharyngeal branch, which is reflected upon the side of the pharynx: whose filaments are- $a$. To the esophagus. b. One received from the sympathetic. $c$. The continuation of the nerve, which is expended in the substance of the pharynx. 3. Two slender branches to the carotid artery, upon the coats of which they ramify, unite, and ultimately split into twigs which form a sort of plexus around the vessel at its bifurcation, from which other filaments are sent along the parietes both of the external and internal carotids. 4. The laryngeal branch, which crosses above the carotid artery, then winds downward upon the side of the pharynx, in its way to the larynx, and enters a perforation through the posterior ala of the thyroid cartilage.

At the posterior part of the neck the par vagum inclines upward, and is found above the carotid artery, between which ressel and the axillary artery it continues its course, through the space between the two first ribs, into the chest. Having entered the thoracic cavity it runs within the superior mediastinum; but it has a somewhat different relative situation on one side from what it has on the other: the right nerve adheres in its passage to the side of the trachea, crosses above the root of the right lung, alongside of the esophagus, and gains the under surface of that tube before it leaves the chest; whereas, on the left side, the nerve accompanies the anterior aorta, and crosises the root of the posterior aorta to reach the esophagus, to the left and upper side of which it pursues its course as far as the the diaphragm. 
Its branches within the chest are-1. Filaments to the trackeal plexus, which consists of an assemblage and intercommunication of nerves, mostly from the sympathetic, around the lower part of the trachea, within the space between the two first ribs. 2. Two or three smaller branches to the cardiac plexus. 3. A single branch, of considerable importance, denominated the recurrent nerve. 4. Branches to the anterior pulmonary plexus. 5. Reflected branches to the posterior pulmonary plexus. And, on the right side, in addition to these, the nerve furnishes a still larger branch which is directed forthwith to the heart; in its passage however it subdivides into two cords, and these branch out as they approach the base of the organ, and penetrate the parietes of the auricles.

The recurrent nerve of the left side originates from the par vagum by the side of the anterior aorta, and coils round the root of the posterior aorta, including that vessel in a sling as it were; but, on the right side, it leaves the trunk as the latter passes the first rib, winding forward within the angle formed between the anterior aorta and its first large branch-the posterior cervical artery. The recurrent nerve, so denominated from its retrograde course, then takes the first part of its reflected passage above and outwardly to the par vagum; by degrees however in running up the neck it approaches the trunk, gains the inner side of it, and along the anterior half of the neck is found between the carotid artery and the trachea. Ilaving reached the top of the air-tube, it spreads into many fine terminating branches, several of which run to the muscles of the larynx, though the principal of them creep along the sides of the thyroid cartilage and end 
in ramifications upon the membrane of the glottis. Its branches are-1. Filaments to the pulmonary plexus; and, on the left side, some also to the cardiac plexus. 2. Filaments to the posterior cervical ganglion. 3. Long slender branches both to the esophagus and trachea in its passage up the neck.

The pulmonary plexuses, inferior and superior, consist of networks of nervous filaments, some of which are of large size, surrounding the great vessels constituting the roots of the lungs. They are coniposed of branches and threads of nerves from the par vagum and recurrent of one side, and of similar ramifications from the opposite side: the inferior plexus clings to the trachea at its division, and spreads under the bronchiæe and pulmonary ressels; the superior, which receives several reflected branches from the par vagum, lies behind the roots of the lungs, and is less considerable than the inferior. From these plexuses the numerous mervous filaments are derived which cling to the ramifications of the bronchiae and pulmonary vessels for the supply of the parenchymatous and vascular structures and lining membrane of the lungs.-Moreover, the pars vagum, in the chest, detaches many twigs to the esophagus, which interlace its muscular coat by uniting with others from the opposite nerve: in this manner another plexus is produced which is named the esophageal.

The par vagum having entered the abdomen, clinging to the sides of the esophagus, the nerve on one side is differently distributed to that on the other. They both run to the stomach; but the left nerve, prior to reaching it, divides into two sets of branches. One of these pursue their course backward and spread 
over the upper part of the organ, sending filaments to the cardia, and others along the small curvature which communicate with the ramifications of the right nerve; the other crosses above the stomach to the left side, taking the course of the gastric artery, and joins the great semilunar ganglion. The right nerve, smaller than the left, as soon as it reaches the cardia, splits into many branches which traverse the small curvature, where they form conmunications with the left, and spread their ramifications upon the under part of the organ: some of them run to the pylorus also, and others go to join the hepatic plexus.

\section{Accessory Nerves to the Eight Pair.}

THESE nerves are considered as accessory to the former in consequence of their being found in close connection in issuing from the cranium. The accessory nerve itself is formed within the vertebral canal, by the concurrence and union of several filaments derived from the side of the medulla spinalis, one or two of which may generally be traced down to the place of origin of the fourth or fifth cervical nerve. In its course into the head it receives many other fine threads from the marrow, and in the cranial cavity joins the par vagum, with which and with the nervus glosso-pharyngeus, all inclosed in one and the same sheath, it descends through the foramen lacerum basis cranii. Beneath the atlas, the accessory nerve leaves its companions and splits into two divisions. - The anterior division runs downward and forward, and penetrates the belly of the sterno-maxillaris, among the fasciculi of which it may be followed, transmitting numerous lateral twigs in its course, down to the very origin of the mus- 
cle. The posterior division turns round the transverse process of the atlas, and takes a waving course between the levator humeri and splenius, obliquely across the side of the neck to the top of the scapula; where it plunges into the rhomboideus brevis, and vanishes in the muscular substance. This division receives filaments of communication from several of the cervical nerves, and furnishes ramifications to the muscles over which it passes. - The branches of the trunk of the accessory nerve are-1. To the par vagum, at its exit from the skull. 2. Some small filaments to the anterior cervical ganglion. 3. A filament of communication with the sub-occipital nerve.

\section{Ninth Pair or Linguales}

Arise, behind the eight pair, from the corpora olivaria, pass out of the cranium through the foramina condyloidea anteriora, and are first found, in company with the par vagum, against the inward sides of the coronoid processes. This nerve takes its course obliquely downward, along the branch of the lower jaw, runs between the pterygoideus and the larynx, and then insinuates itself between the muscles forming the root of the tongue. Having penetrated the substance of this organ, the nerve pervades its middle, accompanied by the lingual artery, and ends in a ramous expansion in its tip. The branches of this nerve are-1. Some small ones in its course, to the hyo-glossus longus; one of which, remarkable for its length, runs to the termination of that muscle, detaching many filaments in its way. 2 . Lateral branches, which come off in a half penniform manner, within the substance of the tongue, and ramify extensively among the fibres of the other lingual muscles. 


\section{LECIUURE LXVII.}

I $\mathbf{N}$ the last lecture I gave the anatomy of those nerves which may be said properly to belong to the head: the nerve next to be described is one that divides its branches, pretty equally, between the head and the neck.

\section{The Sub-occipital Nerve,}

$\mathrm{By}$ some writers considered as the first of the cervical nerves, has a filamentous origin from the place of junction of the medulla oblongata with the medulla spinalis, and passes out through a foramen in the anterior part of the body of the atlas. It then splits into a superior and an inferior division. The superior division, larger than the inferior, we find deeply buried in muscle upon the transverse process of the atlas, where it spreads into several small branches, of which two only are worthy of remark, the others being quickly expended in the contiguous muscles. One of these is a deep muscular branch, which penetrates the mass of muscle upon the occiput; the other ascends over the vertex, and ramifies upon the inward and back part of the ear.

The inferior branch, the longer one, turns round the transverse process of the atlas, and runs through a fo- 
ramen in the anterior wing of it; it then winds down towards the windpipe, and transmits its ultimate ramifications to the thyroid gland, summit of the trachea, and some lymphatic glands thereabouts, and to the sterno-maxillaris and sterno-thyro-hyoidens. Its filaments are- $a$. Some to the decp-seated muscles in front of the atlas. $b$. Two or three to the anterior cervical ganglion. $c$. One of communication with the lingual nerve.

\section{Cervical Nerves.}

I HAVE already shown in what manner these nerves, which consist of seven pairs, originate from the cervical portion of the spinal marrow, and how they fass out on either side, through the foramina which are formed by the articulation of the vertebræ: I may add here, that each pair of nerves are numbered, by way of distinction, after the vertebra immediately behind which they make their exit; and that every nerve, as soon as it has issued from the spinal canal, becomes divided into a superior and an inferior fasciculus, which are directed to the correspondent parts of the neck.

\section{First Cervical Nerve}

Makes its exit from the spinal canal by that large intervertebral gap which exists between the atlas and vertebra dentata. The superior fasiculus of it consists of several branches; I shall only notice one however which is larger and longer than any of the others, all of which soon penetrate the contiguous deep-seated muscles. This branch is reflected backward upon the side of the second vertebra, and is contimued obliquely upward under the complexus, to which muscle its ramifi- 
cations are distributed. It sends backward a ramus of communication to the second cervical nerve.-I'he inferior fasciculus is composed of two principal branches. The superior branch, more considerable than the inferior, turns forward and becomes superficial. It crosses the transverse process of the atlas, and there sends a subcutaneous ramification along the panniculus, to the angle of the jaw. The main branch is then continued upivard, detaches another-a posterior ramification to the panniculus, and ascends to the root of the ear ; here it ends in several small branches, the shorter of which supply the muscles of that part, while the longer creep up the concha and ramify upon its outward surface. In its course, it communicates with the accessory nerve. The inferior branch passes downward and forward upon the longus colli, to which it sends filaments, leaves that muscle to ramify in the levator humeri, and ultimatcly disappears among the fibres of the panniculus. This brancl has also a communication with the

\section{Second Cervical Nerve.}

THrs is a nerve whose distribution is involved in a degree of complexity in consequence the number and diversity of its ramifications. The superior fasciculus includes three or four branches of considerable size. 1. Is found ramifying over the inward surface of the splenius. 2. Runs to the spinalis colli. 3. To the complexus. 4. To the first cervical nerve, and to the complexus minor, extending to the occiput.-The inferior fasciculus furnishes mostly superficial branches. 1. One turns forward and communicates with the accessory nerve. 2. Winds backward and ramifies superficially over the splenius. 3. Pierces the levator humeri, and expends 
itself in subcutaneous ramifications. 4. Creeps along the under surface of the levator humeri, sending off one or two long subcutaneous filaments. 5. Is a filament of communication with the

\section{Third Cervical Nerve.}

Turs is also a nerve whose muscular branches are numerous and intricate. The superior fasciculus is split at its exit into several large and some small branches. 1. Some short twigs to the spinalis colli. 2. A large branch to the splenius. 3. and 4. Long winding branches, which run upon the ligamentum subflavum and vanish in the substance of the complexus. 5. A single branch to the spinalis colli. - The inferior fasciculus is composed of two principal branches. The inferior branch turns round the articulation formed between the third and fourth vertebre, and spreads its ramifications upon the longus colli. The superior branch winds upward for a short way, and subdivides into three smaller ones, of which two run to the levator humeri, and the third pierces that muscle and ends in subcutaneous ramifications: from one of the former branches a filament of communication runs to the

\section{Fourth Cervical Nerve.}

Irs superior fasciculus turns round the vertebre and separates into three or four large branches which supply the contiguous parts of the spinalis colli, splenius, and complexus. - The inferior fasciculus, the more considerable and important one, gives off at its origin some twigs to the longus colli; it then runs down a short distance and detaches-1. A large branch to the splenius. 2. A small one to the longus colli. 3. A lones 
filament which passes backward, covered by the levator humeri, to assist in composing the phrenic nerve. 4. Several long terminating branches, which may be traced upon the levator humeri, over the point of the shoulder. This nerve communicates with the fifth cervical by a small circumflex filament, and sends a considerable branch backward which also joins it.

\section{Fifth Cervical Nerve}

MAKES its appearance between the fifth and sixth vertebre. Its superior fasciculus forms a set of deep muscular branches which are mostly destined to the splenius. The inferior fasciculus comprehends three principal branches. 1. Runs to the point of the shoulder, where its ramifications are principally expended in the levator humeri : at its origin it sends a twig to the longus colli. 2. A large branch to the serratus magnus. 3. The most important of the three, takes a dcep-seated course to the point of the shoulder: there it sends off- $a$. A small branch to the phrenic nerve. $\ell$. One of communication to the sixth cervical nerve. c. One that descends obliquely outward to contribute to the formation of the humeral plexus.

\section{Sixth Cervical Nerve}

Aт its exit, divides. The superior fasciculus, comparatively small, turns round the articulation, runs upward and penetrates the serratus magnus. - The inferior fusciculus, renarkable for the number and large size of its branches, passes backward under the transverse process of the seventh vertebra. It furnishes-1. Some twigs to the longus colli. 2. A long filament to the posterior cercical ganglion. 3. A filament to the phrenic 
282 Seventh Cervical and Diaphleragmatic Nerves.

nerve. 4. Large branches running to the formation of the humeral plexus.

\section{Seventh Cervical Nerve.}

THE last of these nerves comes out between the seventh cervical vertebra and the first dorsal. Its superior fasciculus, very inconsiderable, consists only of a small branch which is expended in the serratus magnus. -The inferior fasciculus comprises a large, flat nerve, which is directed backward to join the humeral plexus. In its way it forms a broad union with the first of the dorsal nerves, and detaches a branch to the sympathetic nerve.

\section{The Diaphragmatic or Phrenic Nerve,}

Principally formed by the union of branches from the fifth and sixth cervical nerves, and generally by the addition of a small filament from the fourth, takes a solitary course down the neck, along the inferior border of the scalenus, enters the chest just above the root of the axillary artery, and traverses the side of the pericardium to reach the tendinous centre of the diaphragm, where it is divided and spread out into many divergent ramifications.

\section{Dorsal Nerves.}

Or these nerves there are eighteen pairs. In issuing from the marrow they are directed obliquely backward. Like the cervical nerves, they are regularly numbered in succession; and present, at their exit, superior and inferior branches; but these branches are less in size, and evolve fewer ramifications. The inferior branches, commonly called the intercostal nerves, run, in company 
with the intercostal blood-vessels, along shallow furrows in the posterior edges of the ribs, between the internal and external intercostal muscles, and direct their course to the sternum.

The superior branches turn immediately upward between the transverse processes, pierce the intercostal muscles and mount upon the back; here they proceed obliquely upward, deeply buried in muscle, at length grow very small and become superficial, and end in subcutaneous ramifications upon the faschia of the back. These nerves grow smaller as they are given off' more posteriorly. The anterior divisions of them furnish branches to the muscles of the dorso-scapular region; the middle and posterior, to the muscles of the dorsal region: the hinder nerves also send a few filaments to the loins.

THE INTERCOSTAL NERVES require that we enter a little into particulars. The

First nerve is of large size, and its principal portion is destined to the humeral plexus. The proper intercostal nerve is but a slender branch. It is remarkable for its solitary course: the other intercostals, for the most part, accompany the intercostal vessels, whcreas this maintains a middle course between the first and second ribs, and ramifies upon their lower extremities. It is also distinguishable from all others but the second, in not furnishing any cutaneous ramification.

The second nerve detaches, near its origin, a considerable branch which describes an arch downward in its course over the belly of the longus colli, and afterwards joins the first nerve. Its intercostal branch, which is rather larger than the first intercostal nerve, runs for some distance along the muscular space, and then 
accompanies the intercostal blood-vessels, and, like the first, ends in ramifications near the sternum.

The third, fourth, fifth, sixth, and seventh intercostal nerves, furnish, each of them, a long cutaneous filament which ramifies among those muscles of the costal region exterior to the ribs; they then pursue their course downward, incline forward between the cartilages, and expend their extreme ramifications among the muscles of the sternal region.-The other intercostal nerves, with the exception of the last, having reached the lower ends of the ribs, do not continue their course between the cartilages, but quit the intercostal spaces and traverse the under surfaces of the cartilages to the muscular parietes of the abdomen, where we find them pursuing their way in parellel lines, nearly at equal distances from one another, between the transverse and internal oblique muscles, to the rectus, in the substance of which they branch out and are lost. From the thirteenth nerve, and from each of those posterior to it, before they leave the intercostal spaces, comes off a large cutaneous branch, which, deviating from the continuation of the trunk, takes an oblique direction backward, and ramifies extensively upon the aponeurosis of the external oblique muscle.

The eighteenth or last intercostal nerve forms an exception to this general description. It quits the last rib at its origin, and stretches obliquely backward, passing close under the extremity of the first lumbar transverse process. Soon after this it splits into two branches: the smaller one, which is directed backward across the abdomen, at first passes between the external and internal oblique muscles, but afterwards insinuates itself among the fibres of the former, and 
ends in subcutaneous ramifications; the larer one traverses the flank and extends for a considerable way between the internal oblique and transverse muscles.

\section{Lumbar Nerves}

CORRESPOND in number to that of the lumbar vertebra. They issue through the spinal foramina in the loins, and are, at their exit, distinguishable into superior and inferior divisions. The superior divisions, of minor importance, are reflected upward and sent into that mass of muscle which forms the loin; and having distributed the majority of their branches to it, they pierce the faschia lumborum and terminate in subcutaneous filaments. The inferior divisions require to be distinctly considered.

The first nerve runs obliquely across the transverse process of the second vertebra, under the sacro-lumbalis, and at the end of the third transverse process divides into two branches. The inner one makes directly for the anterior spine of the ilium, over which it passes, and disperses its last filaments in an arborescent manner upon the iliacus, covered by its faschia. The outer branch takes a sweep outward, between the external and intemal oblique muscles, around the spine of the ileum, and extends along the anterior border of the tensor vaginæ nearly to the stifle, and there ends in subcutaneous ramifications upon the faschia lata. Its other branches are-1. To the last dorsal nerve. 2. To the sympathetic. 3. To the second lumbar nerve.

The second nerve, though generally double from its origin, is smaller than the first. It has communications with the first nerve and sympathetic, and sends 
back a small branch to the formation of the crural nerve. It also sends twigs to the psoæ. Its principal divisions are two. One is stretched along the iliacus outwardly, and continues its passage along the fore part of the haunch, where it becomes subcutaneous and ramifies over the stifle. The other division crosses the ilio-lumbar artery, just below its origin, and takes nearly a similar course to the inward part of the haunch, where it disperses its ramifications upon the skin and faschia: in its way, it detaches a considerable branch -the spermaticus externus, which passes through the abdominal ring, and sends twigs, in the male, to the scrotum, and a filament along the cord to the testicle; in the female, filaments go from it to the uterus, udder, and external labia.

The third nerve is almost wholly consumed in forming the crural nerve. It sends'a small branch to the sympathetic, another to the psoas, and a third backward to the obturator nerve.

The fourth nerve detaches a filament of communication to the sympathetic, and then joins the others in contributing to the production of the crural nerve; but it also sends a considerable branch to the obturator nerve.

The fifth nerve communicates with the sympathetic, and then subdivides into three considerable branches: one contributes to the formation of the crural, another goes to the obturator, and a third splits and sends one portion to the gluteal but a much larger one to the sciatic nerve.

\section{Sacral Nerves.}

OF these nerves we reckon five pairs. The anterior 
nerves are of very large size; the two last are much diminished in volume: they all take an oblique direction backward, and traverse the lateral parietes of the pelvis.

To facilitate their description, we may consider these nerves also as consisting of superior and inferior fasciculi.

The superior fasciculi make their exit through distinct foramina upon the upper part of the sacrum, and are there buried under a thick mass of muscle: a few filaments from them pierce the faschia above and become subcutaneous, and hence extend and ramify upon the outer part of the haunch.

The inferior fasciculi, remarkable for their large size, are of much more importance to us. The first nerve largely contributes to the origin of the sciatic; but it also sends a considerable branch to the gluteal nerve. It communicates with the sympathetic and second lumbar nerve. The second nerve communicates with the third and sympathetic, and then sends off two large branches. One joins the sciatic; the other continues backward and disappears among the coccyreal muscles. The third nerve has similar connections, and also joins the sciatic; but the major part runs backward, along the side of the pelvis, and penetrates the muscles of the haunch. The fourth nerve, being similarly connected, is principally destined to the supply of the bladder and rectum, and muscles of the anal region. The fifth nerve runs backward and plunges into the heads of the coccygeal muscles.

\section{Coccygeal Nerves.}

Generally speaking, we find four pairs of them. 
Like the sacral, they issue from the spine by two sets of foramina, and are therefore best considered as divided into superior and inferior sets. The sympathetic not extending thus far, they have no connection with it. In other respects they do not importantly differ from the other spinal nerves.

The superior fasciculi pass out obliquely backward, communicate with the last of the sacral and with one another, distribute many muscular branches, and end in subcutaneous filaments. From the two or three last originates a considerable nervous cord, which penetrates the erector coccygis, and may be traced among its fibres to the extremity of the tail.

The inferior fasciculi likewise communicate with one another. The first nerve receives a branch from the last sacral, and detaches one to the perineum. Posteriorly, they'all end in one common nervous cord, somewhat larger than that above, which runs between the depressor and curvator coccygis, branching out in its course, and ends in filamentous ramifications at the point of the dock. 


\section{LEC'TURE LXVIII.}

\section{Nerves of the Fore Extremity.}

W ITH the exception of some cutaneous filaments ramifying over the point of the shoulder and extending thence to the arm, the fore extremity receives all its nerves, which are comparatively large and numerous, not to add complicated, from the axillary, better named the humeral plexus; and this plexus is constituted, in a manner that has already been shown, by a small branch from the fifth, by the principal portions of the sixth and seventh cervical nerves, and by the main division of the first dorsal nerve. The plexus, thus formed, resolves itself into several large nervous trunks, generally about seven or eight, which surround the axillary artery, whose particular course and destination I shall now proceed to demonstrate.

THE EXTERNAL THORACIC NERVES, commonly six or seven in number, arise from the humeral plexus, and are dispersed upon the breast and side. Three or four of them run obliquely downward to give branches to the pectoral muscles. The others take a backward course, and send branches to the serratus and latissimus dorsi. One branch is found turning round the posterior border of the triceps, whence it becomes superficial and rami- 
fies among the fibres of the panniculus, rumning thence into the skin.

ThE SCAPULAR NERVES may be distinguished into the anterior, the posterior, and the subscapular nerves.

The anterior scapular nerve is reflected upward and forward, in company with the artery of that name, winds round the anterior costa of the scapula, between the subscapularis and antea-spinatus, to which muscles it sends off branches in crossing the dorsum scapulæ, and stretches its ultimate filaments into the triceps.

The posterior scapular nerve, after detaching a branch to the subscapularis, dips between that muscle and the triceps. There the trunk splits into several branches which run into the triceps, teres minor, and shoulderjoint : one of them is continued upon the outside, round the cervix humeri, where it becomes subcutaneous, and ends in the insertion of the levator humeri.

The subscapular nerves, two, three, or four in number, run upward from the plexus between the scapula and the chest, and enter the substance of the subscapularis.

THE SPIRAL NERVE, the largest of the nervous trunks furnished by the axillary plexus, arises behind the humeral artery, and runs with the vessel for a short distance. It then leaves the artery and turns round the back of the os humeri, between the bone and the large head of the triceps, and is to be found, covered by that muscle, coursing the outward surface of the hody of the same bone. Next it plunges deep among the heads of the extensors; then, much diminished in size, it insiduously creeps round the neck of the radius, and penetrates the head of the flexor metacarpi externus, among the fibres of which its remaining ramifica- 
trons are expended. Its branches are--1. Near its origin, three or four long branches are sent down to the triceps. 2. Lower down, a branch is separated which pierces that muscle and gains the front of the elbow joint, where it escapes through the fischia and takes its course under the skin covering the arm, and ramifies upon the fore and outward part of the knee: this nerve may be distinguished as the external cutaneons nerve. 3. Several considerable branches which penetrate the heads of the extensor muscles.

TIE RADIAL NERVE clings to the humeral artery, with which it descends to the inward side of the elbowjoint, and runs down behind the vessel, along the back of the radius, to the knee; there it passes under the posterior annular ligament, and then continues its course with the artery, and, as soon as it has descended upon the leg, takes the name of the internal metacarpal nerve. Its branches are-1. Which comes off the trunk a little below its origin, from its size and importance is distinguished as the musculo-cutaneous nerze. After accompanying the trunk for a short way, it leaves the parent nerve and crosses obliquely behind the flexor brachii, passing between that muscle and the coracohumeralis, to both of which it sends filaments; it then winds forward and re-appears upon the upper and fore part of the arm, where it becomes subcutaneous, ramifies upon the faschia, and prolongs its ramifications over the knee upon the metacarpus. 2. A small slip detached opposite to the elbow-joint which is distributed to the heads of the flexor muscles. 3. In its course down the arm, several small twigs to the flexor muscles. 4. Above the knee, a communicating slip separates, which obliquely crosses over the fleshy terminations 
of the flexors and joins the ulnar nerve as it passes the joint.

THE ULNAR NERVE which, at its origin from the plexus, holds a middle situation between the radial and the nerve before described - the spiral, also accompanies for a short distance the humeral artery. In its course to the elbow it preserves the line of the os humeri; there it runs over the inward and back part of the joint, glides down the arm concealed by the posterior borders of the flexores metacarpi, inclines a little inward before the tendon of the flexor medius to reach the back of the knee, where it is found under the posterior annular ligament, within a dense faschial sheath, creeping along close to the inner edge of the trapezium. From this, it gains the border of the tendo perforans, and becomes the external metacarpal nerve. Its branches are -1. Soon after its origin, one that may be called the internal cutaneous nerve, which runs over the elbowjoint and ramifies in the loose cellular substance about the inward part of the olecranon. 2. Three or four considerable branches which penetrate the heads of the flexors, are detached also to the joint. 3. A little above the knee comes off a branch which pierces the faschia and becomes subcutaneous. After having detached some twigs to the back part of the knee, this nerve runs along the outer and back part of the cannon to the fetlock, where it disperses its ramifications. 4. A short but considerable branch to the back of the knee, which sends twigs into the joint. 5. A branch is sent off immediately below the knee, which turns suddenly forward and disperses its ramifications in front of the leg.

THE METACARPAL NERVES, internal and external, 
continue down the leg along the borders of the flexor tendons, over the fetlock-joint, where they become the plantar nerves; these pursue their course, behind their corresponding blood-vessels, to the posterior part of the foot, which they penetrate to the inner sides of the lateral cartilages. About midway between the knee and fetlock, the internal nerve sends a branch obliquely over the flexor tendons which joins the external nerve: with this exception, their branches and ultimate destination are the same on either side. Their branches are-l. Filaments dispersed upon the flexor tendons. 2. A large and important branch that comes off just above the fetlock-joint, and runs obliquely forward, and distributes its ramifications to the outward and fore part of the pasterns, and then ramifies upon the coronet.

The plantar nerve detaches-1. A branch opposite to the fetlock-joint that runs, before the trunk, directly to the lateral cartilage, over which its divisions are dispersed. 2. A large branch from the posterior part of the trunk, just before it dips behind the cartilage, which passes backward, and sends its filaments into the fatty frog. 3. Behind the cartilage comes off a branch which winds forward through a foramen in the ala of the coffin bone, to go to supply the laminæ. The trunk then enters the foramen in the posterior concavity of the coffin bone, in company with the plantar artery, and there divides, and distributes its ultimate branches through the foramina around its edge to the sole.

\section{Nerves of the Hind Extremity.}

WE have now to examine the course and distribution of some large nerves which owe their formation to the concurrence and union of several of the lumbar and sacral nerves, in a way that has already been 
pointed out; nevertheless, in commencing my descriptions of them, I shall arrain briefly revert to the manner in which they are constituted.

The CRURAL NERVE is derived partly from the second, but principally from the third, fourth, and fifth lumbar nerves. It is concealed at its origin by the pson muscles; shortly afterwards however it makes its appearance under the last transverse process of the loins, which it crosses obliquely, and proceeds directly backward, in a line with the external iliac artery, to the outward side of, and rather higher than, the vessel, but not in contact with it. In crossing the tendinous and fleshy insertions of the psoas magnus and iliacus, it splits into several parts, and these plunge into the thigh between the rectus and vastus internus, distributing their branches laterally to them and to the vastus externus behind them. Its branches are1. Which comes off at its root and runs still more obliquely outward than the trunk, and distributes its filaments to the iliacus, psoas magnus, and pectineus. 2. The most considerable and important branch takes its course along with the trunk, between it and the artery; but, instead of leaving the vessel, continues to accompany it for a short distance below the pubes; it then emerges forward upon the faschia lata, and divides into two cutaneous filaments: of which, one runs into the stifle and ends in ramifications upon the fore part of the thigh; the other is continued down, in company with the vena saphena, distributing twigs to the skin covering the inward and anterior part of the thigh and leg, where it often splits into two branches, and is traceable with the vein as low as the fetlock.

THE OBTURATOR NERVE, contributed to by the, 
third, but principally formed by the fourth lumbar nerve, sweeps round the brim of the pelvis, first above, and then to the inner side of, the external iliac artery, and passes through the anterior nook of the foramen magnum ischii, detaching some twigs to the obturator muscles in its passage, and is then found sunk in the haunch, behind the pectineus, but before the short and long heads of the triceps, to which muscles it gives branches, and afterwards creeps under the gracilis, upon the inner surface of which it branches out and expends its ultimate filaments.

THE GLUTEAL NERVE receives a tributory ramus from the last lumbar, but owes its formation principally to the first sacral nerve. It leaves the cavity of the pelvis through the foramen in the anterior part of the sacro-sciatic ligament, and winds round upon the dorsum ilii, in company with the gluteal artery, and is entirely expended in the substance of the gluteus maximus.

The scratic nerve, the largest in the body, having derived its origin from the last of the lumbar and three anterior of the sacral nerves, immediately after its formation quits the cavity of the pelvis through a hole in the anterior part of the sacro-sciatic ligament, proceeds backward in contact with that ligament, passes between the hip-joint and the tuberosity of the ischium, and plunges deep into the substance of the haunch. Here we find it split into three large branches-the popliteal nerves, which are lodged in an inter-muscular hollow, bedded in adipose membrane, having the semitendinosus and semimembranosus posteriorly, the biceps to the outward 
side, and the large head of the triceps to the inward side : in passing through the foramen it detaches three or four branches to the head of the biceps, about the same number to the semitendinosus, and two or three to the semimembranosus. Of the three large nerves into which the trunk divides-The first and principal one takes an oblique course between the bellies of the gastrocnemii, leaves those muscles where they become tendinous, and runs to the hock between their tendons and the muscles of the deep posterior crural region, clinging to the faschia which envelopes the latter. At the hock it separates into two nerves-the internal and external metatarsal nerves: the former runs over the tendon of the flexor pedis, and upon the leg creeps along the inward and anterior border of the flexor tendons; the latter passes between the tendon and the base of the os calcis, and pursues a like course upon the outer side. Their subsequent course and ultimate distribution are the same as those of the plantar nerves of the fore extremity. Its branches are-1. A long branch which at first runs with the popliteal trunks, then passes between the gastrocnemius externus and lower end of the semimembranosus, and makes its appearance upon the faschia covering the outerside of the thigh, where it sends off many cutaneous filaments, and ramifies over the outer part of the hock. 2. A long slender branch to the gastrocnemius internus. 3. Several filaments to the skin and faschia, above and about the hock.

The second popliteal nerve passes also between the bellies of the gastrocnemii, above the first, detaching twigs to them in its passage, and then spreads into 
many branches which penetrate the heads of the flexor muscles of the foot, and send filaments into the stiflejoint.

The third popliteal nerve, leaving the others, winds round to the outer part of the thigh, between the gastrocnemius externus and the semimembranosus; there it crosses the head of the peroneus, and then suddenly turns down, running between that muscle and the extensor pedis, along with the anterior tibial artery, and takes its course with it to the middle of the cannon, whence, instead of accompanying that ressel between the metatarsal bones, it pursues its way along the side of the large metatarsal bone, over the fetlock-joint, and terminates subcutaneously upon the side of the pastern. Its branches are-1. A small one to the semimembranosus. 2. A filament, or two, to the gastrocnemius externus. 3. A large branch that runs along the peroneus, under the faschia, and ends superficially in front of the hock. 4. Branches to the flexor metatarsi and extensor pedis. 5. Various small subcutaneous filaments in the remainder of its course.

\section{Sympathetic Nerve.}

Tніs nerve, designated sympathetic from the universal influence which it has in the nervous system, is one no less remarkable for its vast and vital importance in the animal economy than it is for its extensive distribution all over the body, from the head to the tail, for its ganglia, for its plexuses, the number and complication of its branches, and its frequent intercourse and connexion with other nerves belonging to the head, neck, chest, abdomen, and pelvis. Indeed, late experimental researches lead us to consider it as a nervous system of itself, or at least one that renders 
the parts to which it furnishes nerves, independent organs, less under the influence of the sensorium, and altogether free from the controul of the will. The sympathetic nerve may be said to take its beginning from an oblong reddish body, which is found at the base of the cranium, beneath and in front of the atlas, denominated the anterior cervical ganglion. I would observe here however, that the several nerves connected with the ganglia, (however I may treat of them in my descriptions) may be considered either as emanating from the substance of the ganglion, or as contributing to its formation.

The anterior cervical ganglion may be said to be formed principally by two branches which are sent down from the fifth pair, the larger of which accompanies the internal carotid; by two or more fine filaments which are traceable to the edges of the petrous portion of the temporal bone, and are probably derived from the sixth pair; and by two or three other nervous threads accompanying the eighth pair, which seem to spring from the medulla oblongata*. These branches then, we will say, it receives. It transmits filaments

* I am by no means satisfied however about the origin of these threads of nerves, which are so fine that I have not hitherto been able to follow them distinctly through the dura mater, whose fibres so much resemble the nerves themselves. Of the two branches, said to come from the fifth pair, Girard traces the larger one to a ganglion under the occiput, opposite to the origin of the Eustachian tube, which he calls the sub-ocripital ganglion; the other, he says, joins a little ganglion in the carernous sinus. From the sub-uccipital ganglion, he traces two or three filaments onward to the fifth pair, at their origin; and one or two, much larger, he finds, which seem, he says, to be destined to the medulla ublongrata. Vide Giraro's Anal. Gen. Edit. II. Tom, II. pace 1:? 
of communication to the par vagum and its laryngeal branch, to the glosso-pharyngeus, the accessory, and the inferior branches of the sub-occipital and first cervical nerves. Posteriorly, it sends off a filament which crosses to the carotid artery, where it divides and anastomoses with the carotid branches of the par vagum. At its posterior extremity the ganglion grows smaller and ends in the sympathetic nerve, which, at first, has the appearance of being a continuation of it. At its origin, this nerve is deeply lodged between the carotid artery and par vagum; it takes its course along the neck likewise between then ; incleed, it is so closely united with the latter that, being invested in the same cellular sheath, at first view they appear but as one nerve: they are readily distinguished, however, in being disunited, by the comparative small size of the sympathetic. At the bottom of the neck, this nerve lies within the angle formed between the carotid and vertebral arteries; it then runs outward above the vertebral artery, over which we find a bulbous enlargement of it, which takes the name of the posterior cervical ganglion. The only branches given off by the cervical trunk of the sympathetic, are several threads of communication which it has with the par vagum, as they proceed backward together.

The posterior cervical ganglion, smaller than the allterior, is formed under the first dorsal vertebra. From it pass-1. A large branch, and sometimes a smaller one with it, to the sixth cervical nerve, by which it has a mediate communication with the five anterior cervical nerves. 2. A branch to the seventh cervical nerve. 3. A filament to the first dursal nerve. 
4. Another to the second dorsal nerve. 5. Branches to the tracheal plexus. 6. Filaments to the recurrent nerve. 7. To the cardiac plexus.

THE TRACHEAL PLEXUS is an assemblage and intercommunication of nerves, furnished by the par vagum and recurrent as well as the sympathetic, which ramify over the under surface of the trachea as it passes between the two first ribs, from which filaments are transmitted to the cardiac plexus. It supplies this part of the air-tube, and also the contiguous portion of the esophagus.

ThE CARDIAC PLEXUS, constituted of larger branches from the same nervous trunks, and also of filaments from the tracheal plexus, which are readily traceable to the roots of the large bloodvessels, is formed for the supply of the heart.

From the posterior cervical ganglion, the sympathetic continues its passage backward, under the articulations of the ribs with the spine, to the diaphragm, which it pierces in company with the posterior aorta. Between the heads of the ribs it presents little knots-dorsal ganglia, which correspond in number to the intercostal spaces. These ganglia are of very small size when compared with the cervical, and give off, each of them, two filaments to every intercostal nerve.-As the sympathetic nerve pursues its route along the spine, it grows flat, spreads out its fibres, and at length separates into two branches, one of which, by means of frequent reinforcements from the ganglia as it passes by them, ultimately attains a larger size than the original parent nerve: this, from being destined to supply the abdominal viscera, is named the greater SPLANCHNIC nerve. Before it 
leaves the chest, while running along the crus of the diaphragm, the sympathetic detaches another branch, which turns backward upon the crus in the form of an arch in escaping from the cavity, denominated the lesser, secondary, or accessory splanchnic nerve, whose destination is nearly similar to that of the former.

These three nerves, the two splanchnics and the sympathetic, make their entry into the abdomen along the under side of the crus of the diaphragm: the splanchnics are then thus distributed. The greater splanchnic turns downward, and contributes its branches to the formation of the semilunar ganglion; the lesser splanchnic likewise sends most of its branches to that ganglion, but it also transmits two or three long filaments backward to the renal plexus: and both the splanchnics detach communicating filaments to the sympathetic.

The semilunar ganglion, then, is constituted of branches from the left division of the par vagum, and of almost all those of the splanchnic divisions of the sympathetic. This ganglion partakes of the semilunar figure, lies close under the posterior aorta, at the root of the coliac artery, and occupies the space between that vessel and the anterior mesenteric artery. It consists of a number of small ganglia, connected one to another, and is surrounded by a plexus of nervous filaments; which little ganglia have been denominated the coliac. From the irregular convex border of the semilunar ganglion, nervous filaments shoot in various directions, which, from having been compared to the rays of the sun, have been denominated altogether the solar plexus; from which divergent filaments, the several minor plexuses of the abdomen may be said to derive their for- 
mation, taking names according to the viscera they are particularly designed to furnish with nerves. The first I shall notice is

The splenic plexus, which is a network of small nerves surrounding the splenic artery. It sends some filaments to the pancreas, but the majority of its detachments run to the spleen.

The hepatic plexus consists of a similar nervous interlacement around the hepatic artery: it is very extensive; for it winds round the vessel from its origin to its termination in the porta of the liver. It transmits filaments - 1. Along the phrenic arteries to the diaphragm. 2. To the stomach. 3. To the pancreas. 4. It then divides into right and left hepatic plexuses, which cling: to the arteries of the same names, and accompany them in their ramifications through the liver.

The anterior mesenteric plexus is a vast assemblage of nervous filaments, intricately wound round the trunks of the anterior mesenteric arteries, which furnish numerous filaments that proceed along with these vessels, greatly exceeding them in number, to ramify within the substance of the small intestines, the cocum, and coecal portion of the colon.

The uortic plexus, a name given to the frequent intercommunication of some fine filaments which are sent backward along the posterior aorta from the solar plexus, with two or three contributory branches from the trunk of the sympathetic. Most of the filaments derived from it, pass on to form

The posterior mesenteric plexus, one that is likewise re-inforced by branches, which are comparatively large in size, from the sympathetic. It transmits nerves to that part of the colon left unsupplied by the anterior 
mesenteric plexus, and to the rectum: some few of them also ramify in the substance of the mesocolon, and others run into the lynphatic glands seated within the folds of that membrane.

The hypogastric plexus, removed still further backward than the posterior mesenteric plexus, consists of divers delicate nervous filaments ramifying upon the aorta, and spreading themselves, entangled in cellular substance, about its bifurcation. This plexus is also multiplied by branches coming from the sympathetic. The filaments which depart from it run to the pelvic viscera and the organs of generation.

The renal plexus is a more important one ; it is produced by the intercommunication of a few filaments from the lesser splanchnic nerve with numerous others from the solar plexus. It is found close to the aorta, immediately behind the anterior mesenteric plexus. It conceals the origins of the emulgent arteries, spreads round them in their passage, and sends filaments in company with their branches into the substance of the kidneys. It also furnishes with nerves, the capsulæ renales, the renal glands, and the beginnings of the ureters.

The sympathetic nerve in the abdomen travels over the sides of the bodies of the lumbar vertebræ, below the articulations of the ribs, covered by the crura of the diaphragm, and thus pursues its course into the pelvis. Here likewise it forms ganglia, which correspond in number to that of the lumbar nerves; and from every ganglion come off two filaments: one of which runs to the correspondent lumbar nerve; the other crosses the aorta, and, by joining the aortic plexus, communicates with nerves coming from the sympathetic of the other side. 
From the loins, the sympathetic descends into the pelvis, where it takes its course laterally along the body of the sacrum. Five sacral ganglia, corresponding to the five sacral nerves, with which they communicate, are found here, and between them run in arches across the sacrum several intercommunicating filaments. In quitting this bone, the nerve grows so small, and is so firmly adherent to the ligamentous covering of the os coccygis, that I have not yet succeeded, to my mind, in ascertaining the precise manner in which it finishes its course : it is said, however, to do so by forming a union with its fellow.

*** I take this opportunity of acknowledging the services afforded me in the dissection of the nerves by Mr. BEAN, V.S., who, I believe, is now in practice at Durham. Unwearied assiduity and unusual zeal had qualified him in an especial manner for such an undertaking; and I hope that he is now enjoying that support which his professional acquirements will always render him deserving of. 


\section{LFCIUURE LATX.}

\section{Distribution of Arteries.}

I $\mathrm{T}$ has been observed, in former lectures, that the more perfect animals are formed with two distinct and complete arterial and corresponding venous systems; and that these vascular systems, though both transmit the same blood, have no intercommunication but through the medium of the heart, which, in fact, is a double organ; and that one system is solely employed in the pulmonary circulation, but that the other pervades every other internal organ and extends to the remotest parts of the body: the pulmonary system was described with the lungs; it is now my intention to trace the ramifications of the

\section{Aortic System.}

THESE may be compared, as a whole, to a short but straggling and very branchy shrub or tree of luxuriant but extremely irregular growth; and their number and distribution may be presented in idea to the mind by recalling to memory, that no organized part, the lungs even not altogether excepted, is without few or many of them. To follow the course or give distinct names 
to the very small branches, would answer no useful practical end, while it would heavily encumber our minds to carry such a vocabulary about with us; and though we succeed in a general way in discovering their destinations, to say precisely in what structure or arrangement they ultimately end in too many instances yet needs further demonstration: I shall therefore not dwell on these points of description, (many of which have already been discussed) but direct my observations to those vessels in particular whose size, situation, or connection, may render a knowledge of them important in operations, cases of hemorrhage, or physiological and pathological researches.

The AORTA, the main trunk of the large arterial system, rises out of the base of the left ventricle in a vertical direction in the space between the left auricle and the pulmonary artery, which vessel, by inclining forward as the aorta leans backward, leaves visible on a side view a small angular portion of the latter. The length of the trunk is about two inches; and the ascent takes place opposite to the body of the fourth dorsal vertebra, below which it bifurcates into two curved vessels of unequal dimensions, the aorta anterior and the aorta posterior.

The only branches furnished by the main trunk are the coronary arteries, which arise near its root, just over two of the semilunar valves. The right coronary artery emerges from between the pulmonary artery and the right auricle, winds round the fissure separating that cavity from the right ventricle, and turns down under the termination of the vena cava, withinthe furrow dividing the ventricles, upon the side of the heart, distributing lateral ramifications in its course which pene- 
trate the substance of the parietes, and ending in small spiral branches near the apex cordis. The left coronury artery in passing out between the pulmonary artery and left auricle, sends off a large branch which encircles the other auriculo-ventricular fissure; it then takes its course downward within the ventricular furrow upon the left surface of the heart, distributing lateral branches which supply the parietes forming this side, and ending in spiral ramifications which extend quite around the apex cordis, and anastomose with those upon the opposite side.

\section{Anterior Aorta.}

THE anterior, smaller, and shorter division of the main trunk, whose length falls somewhat short of an inch, in ascending, bends forward, and divides below the body of the third dorsal vertebra into the right and left arteriæ innominatæe vel communes. The course of this vessel is under the windpipe and rather to the left of it, within the space of the mediastinum. The trunk itself furnishes no branches; but its bifurcations give origin to those large arteries which are distributed over the breast, neck, head, brain, and anterior extremities.

\section{Arteria Innominate vel Communes.}

Tн $\mathrm{E}$ right artery is considerably longer than the left, and measures nearly as much again in circumference in consequence of its forming the common carotid artery or the trunk from which the two carotids spring, after it has detached branches to the right side correspondent in size, number, and distribution to those into which the left division resolves itself. These vessels form a 
very acute angle as they leave the anterior aorta, and pursue their course horizontally forward, diverging very gradually as they advance: the right is placed lower than the left, and lies more immediately under the windpipe, having the vena cava anterior upon the other side of it; the left describes a curve upward in passing forward, inclines outward and gets under the esophagus: both branch out about the middle of the space between the two first ribs, where the right ends in the two carotid arteries, the left in the vessels I shall now describe, which, as was observed before, are nearly the same on one side as on the other.

1. THE DORSAL ARTERY generally comes off in one common root with the next vessel. From its origin, it curves upward and backward to perforate the second intercostal space, which it does very obliquely close to the posterior edge of the first rib, a little below its head; out of the chest it pursues the same oblique course, crossing over the transverse process of the second dorsal vertebra, and then ascending upon the spines of the withers, among the muscles attached to which its ramifications are expended. Its branches worthy of names are-a. Superior Mediastinal, a twig detached near its origin to the mediastinum. b. Anterior intercostal, a branch sometimes nearly equal in size to the dorsal itself, given off just before the vessel penetrates the intercostal muscle, by which the second, third, fourth, and fifth intercostal arteries are furnished: the reason for this is, that the posterior aorta, from which all the posterior intercostals spring, is too far removed from these ribs to send vessels to them; their deficiency therefore was conveniently supplied by the anterior intercostal. The intercostal branches follow 
the posterior margins of their correspondent ribs, each running within a shallow groove; midway however between the vertebræ and sternum they leave the ribs for the middle of the intercostal spaces, and end in several slender ramifications, some of which may be traced as low as the sternum, there anastomosing with similar ones coming from the internal pectoral artery. The intercostals in their course detach twigs to the pleura, but more especially supply the intercostal muscles.

2. The Posterior CERVICAL ARTERY, arising in common with the dorsal on the right side, but by a separate root on the left, takes an opposite direction to that vessel, inclining forward and upward. It traverses the upper part of the first intercostal space, piercing the muscle filling it with considerable obliquity, and then ascends in a winding course between the transverse processes of the first dorsal and last cervical vertebræ, upon the body of the latter, where it turns forward, and runs as high as the vertebra dentata, close to the roots of the ligamentum nuchæ, covered by the complexus major to which its ramifications are principally distributed. Besides some small and unimportant branches within the chest, it gives off the first intercostal artery, which descends behind the first rib and anastomoses with twigs from the internal pectoral.

3. The vertebral artery is a vessel of considerable size, and is important from being one of the principal conduits of blood to the brain. It arises with a sort of bend from the upper part of the arteria innominata, immediately opposite to the first rib, so that if a knife were thrust into the chest behind the rib the artery would just escape puncture. The right is at its origin placed lower than the left, and deviates some- 
what less from a straight line in proceeding close under the transverse process of the seventh cervical vertebra to enter the foramen through that of the sixth. They then continue their passage to the head, passing directly through the foramina of the fourth, third, and second vertebræ, wherein they run securely barred from external injury, and, arriving at the atlas, make a curve upward upon its transverse processes, proceed through the posterior pair of foramina, and enter the foramen magnum. In its course along the neck, the vertebral artery detaches several short branches of considerable size which turn round the transverse processes and ramify among the deep-seated muscles. It also sends some smaller ones inward, which enter the intervertebral holes for the supply of the medulla spinalis and its membranes. Under the atlas, the vertebral trunk receives a large vessel of communication from the internal carotid : but I shall suspend its further description until we come to investigate the vessels of the brain.

4. The internal pectoral artery, less in volume than the vertebral, leaves the trunk below, opposite to the origin of that vessel above, protected by the first rib, along the posterior and inner margin of which it descends in a perpendicular direction towards the sternum. Leaving the lower extremity of the rib, it makes a broad sweep downward and backward, and descends upon the internal surface of the second bone of the sternum; it afterwards diverges gradually from its fellow, traverses the ends of the cartilages of the posterior true ribs, and, having crossed that of the last, splits into two divisions. One of these ascends upon the internal part of the chest along the border of the 
seventh true cartilage, thence it crosses the false cartilages, detaching slender ramifications to them which anastomose with some of the posterior intercostals, and makes its way towards the flank, and disperses its ultimate branches among the muscles there, some of which reach far enough to communicate with the ramifications of the circumflex artery of the ileum. The other division, generally considered as the continuation of the trunk, being rather the larger one, pierces the sheath and makes its appearance upon the upper surface of the rectus, over which it ramifies extensively, sending branches out that anastomose with the epigastric. Exclusively of these divisions, the trunk detaches muscular branches along the first rib, inwardly anterior mediastinal vessels, larger branches to the muscles of the sternum, and inferior mediastinal, and also thymic twigs.

5. The external Pectoral artery, comparatively small and unimportant, comes off from the under part of the trunk, makes a turn downward in front of the sternum, and distributes its ramifications among the pectoral muscles: this artery is in some subjects derived from the internal pectoral.

6. The inferior cervical artery, longer and larger than the afore-described vessel, arising from the common trunk opposite to or in company with it, makes its exit from the chest below the vertebral artery, and then bends outward. At the bottom of the neck it divides into several branches: these run principally to the contiguous muscles, though some few ramify within the adipose substance in the neighbourhood, and others are destined to the absorbent glands hereabouts.

7. The seventh and last branch, and largest if we 
except the carotids on the right side and the continuation of the main trunk on the left, is the

\section{Axillary Artery,}

THE source from which spring all THE ARTERIES OF THE FORE EXTREMITY. This vessel is so situated between the scapula and the trunk that not only is it out of the way of all injury, but it cannot be got at for the purpose of demonstration without detaching the limb from the side. It arises within the chest from the arteria innominata, gains exit by making a sudden turn around the first rib, rather below its middle, crossing the lower border of the scalenus in the turn; it is first directed outward in this flexure and then backward, and at length reaches the inner part of the head of the humerus where it makes another turn backward, and afterwards takes the name of brachial artery. Its branches are-1. The external thoracic extends backward across the arches of the ribs, taking the same course as the vessel called the "spur vein," and supplying the muscles thereabouts. 2. The humeral thoracic runs to the point of the shoulder and gives its branches to the levator humeri and shoulder-joint. 3. The dorsaTis scapula ascends in a flexuous manner obliquely upon the shoulder-joint, crossing the insertion of the subscapularis; it then runs for a short way along the anterior costa, around which it afterwards continues to reach the antea-spinatus. 4. I he subscapularis, a large artery which also arises from the upper part of the trunk, but near to its termination. It creeps upward along the posterior costa, screened from view by the edges of the subscapularis and teres major, to both of which muscles it detaches laterally several small branches, 
and ends near the posterior angle of the bone. It gives off a considerable branch at a short distance from its origin which proceeds in a waving line across the inner surface of the triceps, and ends in the panniculus carnosus; and another, a deep-seated one, about the middle of the costa, which is principally expended in the head of the triceps.

THE HUMERAL ARTERY descends from the inner and back part of the head of the os humeri in an oblique direction to the inferior and anterior part of the body of the bone, where it divides into the ulnar, spiral, and radial arteries; on its inner side, it has the spiral and ulnar nerves; in front, the radial nerve; and behind, the humeral veins : and it is covered internally by the pectoralis magnus, to which it sends some small branches. But its principal branches are-1. One near its origin which crosses the bone to get to the flexor brachii, and sends twigs to the shoulder-joint. 2. A posterior branch, arising a little lower down, which bifurcates and enters the triceps. 3. Near its termination, another anterior branch to the flexor brachii. Where the artery divides, it is covered by the humerul plexus of veins, and by the absorbent glands of the arm.

THE ULNAR ARTERY consists of a common root from which spring three or four vessels of considerable size rumning in waving lines upon the inner side of the lower end of the humerus. The upper one, commonly the largest, is directed to the ulnar, splitting however before it reaches the bone, and sending one branch upward upon the olecranon, and another downward to the heads of the flexors; to which muscles the other branches of this vessel are cxclusively distributed. 
THE SPIRAL ARTERY, the outermost division, turns round the os humeri, passing under the flexor brachii and sending a recurrent branch to it, to arrive at the front of the head of the radius, where it splits into several branches of which-1. Some run into the elbowjoint. 2. Others larger and more in number penetrate the heads of the extensors. 3. Two long slender ones descend upon the radius, under the extensor muscles to which they give branches in their course, as low as the front of the knee, and there end in ramifications about and into the joint, anastomosing with others coming from the radial.

The RADial aRTERY, the principal division of the humeral, continues its descent along the radius, inclining as it descends from the inner to the back part of that bone; so that if it were designed to cut down upon it about the middle of the arm, the incision should correspond to the inner edge of the radius, from which the faschia must be first detached, and afterwards the flexor metacarpi internus. The radial nerve, which here accompanies it, runs first upon its outer side and subsequently gets behind it. A short way above the knee, it splits into the metacarpal arteries. It gives off-1. Small vessels to the elbow-joint. 2. Various branches to the flexor muscles during its course. 3. The medullary artery of the radius, about one-fourth of the length of the bone downward.

THE SMALL METACARPAL ARTERY descends, within a cellular sheath, along the inner and back part of the knee, more outwardly situated than the large one, from which it is separated by the posterior annular ligament. It continues its descent along with the metacarpal vein (which runs to its inner side) till it gets 
below the knee, where it bifurcates and transmits its divisions down the front of the suspensary ligamentbetween it and the cannon bone. It sends off- -1 . Cutaneous branches over the front of the knee, which anastomose with the spiral. 2. Ramifications to the front of the cannon. 3. To the suspensary ligament.

THE LARGE METACARPAL ARTERY, which may be regarded as the continuation of the radial trunk, continues it course down the leg by the side of the tendo perforatus, passing with it at the knee under the posterior annular ligament, inclosed within a cellular sheath of its own. As it proceeds, it inclines to the side of the tendo perforans, and in approaching the fetlock, gets in advance of that tendon; just above the joint, where it is situated between the tendo perforans and the suspensary ligament, it splits into three vessels. The middle division gets between the bifurcated terminations of the ligament, and between it and the bone forms a transverse arch, from which three recurrent arteries retrace in a flexuous line the suspensary ligament, and form communications with the small metacarpal artery: the lateral divisions become the plantar arteries. From the arch below come off two lateral branches which descend into the joint.

The plantar arteries, external and internal, in the fore extremity result from the bifurcation of the metacarpal, in the hind from that of the metatarsal artery: I prefer this appellation for them because it denotes their destination at once, and because it saves us the useless division of a trunk only six inches in length into three arteries, and because our descriptions and memories are not clogged by it as they are by the "large pastern," and the "small pastern," and the 
"coffin arteries." Their general distribution is the sanie both in the fore and hind feet.

These arteries, in emerging from their origin behind the flexor tendons, form the two sides of a triangle, the base of which, turned downward, is represented by a line drawn across the backs of the sesamoid bones, the apex being the point of bifurcation. They descend the fetlock upon the sides of the sesamoids, in company with their viens which run in front of them, and with the plantar nerves which proceed behind them; in this part of their course they describe corresponding curves outward to conform to the prominence of the fetlock, and henceforward pursue the same uniformity in course and distribution, so that we need only for the future make mention of one. In its passage over the sesamoid, the outer edge of that bone rose between it and the flexor tendon, but in quitting the fetlock, the artery again approaches the tendo perforatus and runs beside it to its termination in the head of the os coronæ, at which place the artery sinks behind the cartilage into the substance of what is called the "fatty frog." Inclining forward in its subsequent descent, the artery next passes the inner and upper extremity of the ala of the os pedis, where it enters a groove scooped in that bone which conducts it, obliquely forward and inward, to the foramen in the posterior concavity of the bone. Here we lose sight of it altogether-the knife and forceps no longer avail us to discover its progress and destination: either the bone should be chiselled away or (the vessels being previously injected) be croded by maceration in an acid; we shall then detect the artery in the interior of the coffin-bone, making a turn outward again, and subsequently another 
inward, in the course of which it meets with its fellow; the trunks then coalesce, and in so doing form an arterial semicircle corresponding to the circumferent line of the edge of the os pedis, which has been very properly named by the Professor, the circulus arteriosus*. The plantar vessels and nerves are invested in their descent to the foot by cellular substance, which bind them loosely to the parts contiguous to which they pass; this accounts for their canals being flexuous when distended with injection or when the foot is flexed upon the fetlock : a circumstance that seems to have escaped the notice of writers on the foot. The branches of the plantar artery are many and important. After detaching some small ramifications inwardly to the fetlock, posteriorly to the flexor tendons which anastomose with their fellows, and anteriorly to the extensor tendon which are also anastomotic, it sends off-

1. The perpendicular artery, a little above the middle of the os suffraginis : this slender branch descends upon the side of the bone, inclining forward, and ends over the coronary ligament in anastomosis with its fellow, forming an arch, presenting its convexity downward, called the superficial coronary, from which emanate about eighteen small descending arteries that run directly over the coronary plexus of veins, whose principal function it is said is "to secrete the crust." I would remark here however, that, although I believe what I give to be the general arrangement of these arteries, there is so much variation that I cannot vouch for the correctness of this description, however I may stand amenable for its accuracy. The trunk

* Professor Coleman, "On the. Font of the Horse." Vol. II. 
having detached various small unimportant branches backward, one great design of which appears to have been to preserve free and continual intercouse with the opposite trunk, it next sends off, below the pastern-joint.-

2. The transverse artery, which proceeds directly across the front of the os coronæ, underneath the extensor tendon, to join the corresponding branch from the other side, the two together forming the superior coronary circle: this pours most of its blood through two short lateral conduits, the communicating arteries, into the inferior coronary artery; other short twigs are sent both upward and downward from it. The two that have been described may be considered as the anterior branches of importance; let us now proceed to those arising posteriorly: the first we need notice particularly is-

3. The artery of the frog. It comes off opposite to the pastern-joint, and descends obliquely inward through the substance of the fatty frog, wherein it bifurcates: both divisions take nearly the same direction, one passing down upon the side of the cleft along which it continues, distributing branches in its course to the toe of the frog, and forming communications with the vessels of the sole; the other ramifies over the heel of the frog, and sends branches outward to the cartilages.

4. The lateral laminal artery leaves the trunk just as the latter reaches the os pedis, passes through the foramen in the ala, and proceeds within a superficial groove to the front of the foot, distributing branches upward and downward to the laminæ, and disappearing through a small foramen in the anterior and lateral part of 
the coffin, which it enters to form a communication with the circulus arteriosus. From this vessel, a branch descends upon the side of the bone to join the circumflex artery.

5. The circulus arteriosus, resulting from the coalition of the main trunks, preserves to a certain extent around the toe of the coffin the same curve interiorly that is made by the exterior edge of the bone itself, at the distance of about an inch above it. From it arise two principal sets of vessels.

1. The anterior laminal arteries, numerous, small, short branches springing from the front of the circulus, and making their exit through the various foramina in the front and sides of the coffin to ramify among the laminæ, anastomosing with the descending and lateral coffin arteries.

2. The inferior communicating arteries, "thirteen, and sometimes fourteen" in number, according to the Professor, arise from the convexity of the circulus arteriosus, and descend through the foramina in front of the coffin, a little above its boundary edge; having made their exit, they continue the same direction to gain this edge around which they are all received by the-

3. Circumflex artery, which is commonly described as encircling "the toe" of the coffin-bone. Then, again, from this vessel spring-

The solar arteries, (which may be so named from their radiated arrangement,) thirteen or fourteen in number, which, though they run from the same channel, do not "take their origin immediately opposite the termination of the vessels into the circulus arteriosus." These vessels are destined for the supply 
of the sole, upon which they run in radii, at pretty equal distances, whose common centre is the toe of the frog, where they end in communications with the alteries of that body.

One great and evident design in the distribution of arteries, is, so to direct them in their course that they may be out of the way of injury and pressure, or else, so to arrange their communications that the pressure to which they are on occasions unavoidably subjected may not altogether arrest the circulation. No part of the body exhibits a more striking illustration of the latter than the artérial arrangement we have just been examining. This object indeed seems to have been carried further in the foot than elsewhere; the ultimatum being rather to furnish an unceasing flow of blood (and an equable secretion of horn consequent on it) than to provide against unavoidable or accidental impediment, a point that has been argued in a very convincing manner by the Professor in his observations on the foot. I do not however concur altogether in opinion with this ingenious physiologist when he infers, that this arrangement of the arteries "must impede the passage of the blood, and render the circulation slower:" certainly, "on mechanical principles," such would be the result; but we must remember that the arteries are not passive tubes, indeed there appears reason to believe, on the contrary, that they themselves take a very active part in the circulation, and if such can be shown to be the case (knowing that they are constantly filled with blood) their distribution or arrangement can have, I apprehend, no such effect: in opening the circumflex artery I have invariably found that the blood flowed from it as fast as 
from any other vessel of the same diameter in the body. - Furthermore, the Professor justly remarks, that the circulus arteriosus is "more complete than is found in any part either of the horse or the human subject. If one trunk be totally stopped, the circle below will not be distended with the same force, but as completely filled as before."-That " the anterior coffin (circumflex) artery must be perpetually filled with blood, so long as one vessel remains entire."-And that the arteries pass through the substance of the os pedis "to prevent pressure on them;" but "if the veins had returned their blood through it, the bone must have been made so porous as to have rendered it too weak to support the weight of the animal." 


\section{LECTURE LXX.}

\section{The Carotid Artery.}

THE right arteria innominata, having detached seven important branches which vary but little in their mode of origin, and not at all in their general course and distribution, from the seven arteries into which the left division resolves itself, becomes the common carotid, a large vessel about an inch in length, emerging through the upper part of the anterior opening of the chest, having the trachea between it and the spine above, the vena cava anterior below it, and dividing as it quits the cavity into the right and left carotids. These arteries bend upward from their origin, diverging as they mount the neck so as to leave a space between them for the windpipe; having climbed the sides of which, they closely embrace and cling to it for the first part of their course; towards the middle of the neck however we find them gradually inclining, as they ascend, to its posterior and lateral borders, the line of direction of which they preserve during the remainder of their course*. Having reached the top of the larynx, the

* The carotids lie more deeply the higher they proceed up the 
carotid of either side splits into three divisions:-the external and internal carotids, and the ramus anastomoticus: here, though the trunk itself becomes deeply lodged in soft parts, its situation is well indicated by the larynx, with which it is in contact, below, the transverse process of the atlas above, the angle of the jaw a little in advance of it, and the coronoid process farther removed above and before it: a deep incision corresponding to the posterior border of the stylo-maxillaris, would sever the artery at or very close to its division. This vessel detaches-1. Several unimportant muscular branches in its progress up the neck. 2. The thyroideal artery, coming off opposite to the top ring of the trachea; a branch of no mean size which turns round the windpipe and enters the substance of the thyroid gland. In its way, it furnishes the laryngeal, a small artery that perforates the ligament uniting the cricoid and thyroid cartilages, and is dispersed upon the membrane lining the larynx: in some instances this forms a branch of the main trunk. The common division of the carotid is into the three vessels just named; but not infrequently we meei with a fourth coming from the point of ramification, the additional one being the facial artery; and occasionally we only find a simple bifurcation of the vessel into the external and internal carotids.

neck: each is covered by the sterno-maxillaris; and the readiest way to find the vessel is to make a cut along the upper border of this muscle, and depress it with the finger or handle of the knife. The artery is separated, except at the bottom of the neck, from the jugular vein by a thin partition of muscular fibres; the par vagum accompanies it outwardly, and the sympathetic nerve runs between the two. 


\section{The External Carotid Artery}

Is the large division, which may justly be regarded as the continuation of the trunk of the common carotid itself. It takes a flexuous course, first curving downward behind the angle of the jaw, where it crosses the insertion of the stylo-maxillaris, by which it is here defended from injury; it next makes a curve upward and forward, crosses the membranous sac of the fauces, and passes between the stylo-maxillaris and cornu of the os hyoides, deeply buried under the parotid gland; it then makes a third curve which directs it forward, along the posterior border of the branch of the jaw, and to that it afterwards corresponds until it bifurcates, which it does immediately behind the neck of the condyle. This artery is so bedded in glandular substance, surrounded by venous and nervous trunks, and protected by neighbouring bony prominences and muscles, that but a small, and that the upper, portion of it is safely accessible to the knife : rather more than an inch below and behind the condyle, it is comparatively superficially lodged, being there only covered by the anterior thin border of the parotid and a thick aponeurosis, in addition to the common integuments. The first branch of the external carotid is the

1. Submaxillary artery: it comes off behind the cornu of the os hyoides, just as the carotid is going to make its second curve, and ranks next in size to the trunk itself. It takes an oblique course downward and forward within the sub-maxillary space, preserving at first the line of the cornu; it then crosses the lower portion of the pterygoideus, and reaches the posterior border of the branch of the jaw, about one-third of its length downward, which it turns round to arrive upon 
the face: here it becomes subcutaneous, distinctly perceptible to the feel, and very conveniently compressible, on which account it is the vessel ordinarily selected to convey a knowledge of the state of the pulse. In ascending upon the side of the jaw, the artery corresponds to the anterior border of the masseter; it does not reach however the alveolar processes before it ends, by a pretty equal division, in the facial and inferior labial arteries.-Its branches are- $a$. The ascending pharyngeal, which mounts obliquely over the cornu of the os hyoides, and ramifies upon the side of the pharynx, giving off commonly a laryngeal twig or two in its way, and another to the velum, which in some instances is derived from the submaxillary itself. $\quad b$. Various inconsiderable branches to the pterygoid muscle and parotid gland. c. The lingual, nearly or quite equal in magnitude to the submaxillary itself. It first bends its course obliquely inward, detaching a few twigs into the submaxillary space; it then splits into two arteries, the ranine and the sublingual. The ranine, the larger one, the apparent continuation of the lingual, turns downward and proceeds in a flexuous manner along the under part of the tongue, serpentining between the muscles and transmitting many branches into the interior; it continues of large size even to the tip of the organ, wherein its extreme ramifications are expended. Its ramifications have no anastomosis in ordinary cases with those of its fellow of the other side; though I have a head before me in which the two ranine arteries communicate by a large cross branch at the root of the tongue. The sublingual artery winds along the under and outer border of the tongue, preserving a more superficial course than the former. 
It supplies the sublingual gland, and distributes its longer branches over the membrane and papillæ of the tongue. $d$. The submental artery leaves the submaxillary contiguously to the internal side of the jaw, a little before the latter vessel begins to make its turn. It follows the course of the branch of the jaw, nearly preserving the line of its middle, detaching twigs principally to the pterygoideus and mylo-hyoideus, and transmits its furthest ramifications into the substance of the gums internally. e. Anterior masseter branches, one large, or two or three small vessels, coming off in the course of its ascent upon the external side of the jaw.

THE INFERIOR LABIAL ARTERY courses the side of the jaw, occupying nearly the same site externally to what the submental does internally, invested in the cellular and fleshy substance belonging to the buccinator. It is principally destined for the supply of the glandular substance of the under lip, wherein it anastomoses with the corresponding vessel. It gives offa. Slender ramifications to the investing cellular substance. b. Buccinator arteries. c. A large branch to the angle of the mouth, which distributes buccal twigs in its course, and then bifurcates, sending its divisions respectively to the upper and under lips, along their lateral borders: these form the superior and inferior coronary arteries of the lips.

THE FACIAL ARTERY ascends upon the side of the face, with an inclination forward and downward, crossing the buccinator a little in advance of the anterior border of the masseter. Having run as high as the level of the bony ridge from which the masseter arises, it detaches a large branch, and then winds upward and spreads into an arborescent expansion upon the upper 
and fore part of the face. $a$. It mostly sends one or two musseter branches backward. b. Buccal twigs from both sides. $c$. The superior labial, the branch whose origin has just been shown, takes its course below the false nostril, to which it sends ramifications, to the upper lip, wherein it anastomoses with the terminating branches of the palatine arteries and with its fellow. d. Long slender branches, commonly two, to the false nostrils. e. Terminating ramifications to the cellular substance and skin covering the fore part of the face, which anastomose with others making their exit from the infra-orbitar foramen, and also with some straggling twigs escaping from the cavity of the orbit.

2. The Parotideal branches are those we may consider next to the submaxillary branch of the external carotid. They are too variable in number, size, and mode of origin, to admit of particular descriptions : they come off as the vessel continues its course under the gland.

3. A large branch, internally, to the pterygoideus.

4. One much longer, externally, the posterior masseter artery.

5. THE POSTERIOR AURICUlar commonly comes off immediately opposite to the last-mentioned branch. It emerges from underneath the parotid gland, and ascends in a direct line to the back of the concha of the ear, where it splits into three divisions, which thence proceed along its dorsum to its tip, distributing branches right and left which anastomose freely with one another and the other auricular arteries, and thereby form a beautiful vascular network. It gives branches to the parotid gland, muscles of the concha, meatus auditorius externus, and membrana tympani. 
6. THE TEMPORAL ARTERY, the anterior auricular, and the internal maxillary, may be considered as the terminating branches of the external carotid. The temporal leaves the trunk just as it is emerging from the depth of the parotid gland, curves upward and forward around the neck of the jaw, a little below the condyle, which serves as a guide to cut down upon it; from this, it runs in a straight line towards the outer circumference of the orbit, just opposite to which margin it dips into the substance of the masseter so as to elude further trace without the aid of dissection. We find it henceforward corresponding in course to the line of the maxillary ridge, sending branches down to the muscle, and freely anastomosing with the anterior and internal masseter arteries.

7. THE ANTERIOR AURICULAR ARTERY, arising in common with the former, inclines in an opposite direction, upward and backward, and is deeply seated at first under the parotid gland. It ascends to the fore part of the root of the ear, and ends in short ramifications to the anterior muscles of the concha, and in other superficial ones which anastomose with the anterior auricular arteries. It sends off- $a$. Branches to the temporal muscle. b. An internal auricular branch, which enters the concha. c. A subcutaneous branch, which descends upon the forehead and anastomoses with the supra-orbitar arteries.

8. THE INTERNAL MAXILLARY ARTERY is deeply placed to the inside of the articulation of the jaw, a little below the condyle, between the neck of the inferior maxilla and the upper extremity of the cornu of the os hyoides. It pursues a winding course, first bending inward then downward, to the bottom of the 
orbit, where it separates into four arteries. In its way to the orbit, it gives off-1. Deep temporal branches, several small arteries, variable in number, some of which run into the pterygoid muscle, while others, longer ones, ascend in the space behind the orbit, ramifying within the adipose matter there, and penetrating the lower portion of the temporal muscle.

2. Long slender twigs to the soft palate, to the ear, and to the articulation of the jaw.

3. The inferior maxillary, a small artery of considerable length, which creeps down the branch of the jaw, crossing the pterygoid muscle, to enter the foramen maxillare superius in company with the nerve of the same name; within which canal it distributes branches to the roots of the molar teeth and to the diploe, and then makes its exit, greatly diminished in size, through the foramen maxillare inferius, upon the side of the mouth, where it is lost in anastomosis with the inferior labial artery: in some instances, the latter artery sends a twig into this hole in place of one coming out. The vessels in which the internal maxillary terminates are-

4. The supra-ortitar artery, which traverses the upper and inner part of the roof of the orbit, and leaves the cavity through the foramen supra-orbitarium, and is lost in slender ramifications in the cellular membrane upon the forehead, anastomosing with the temporal branches of the anterior auricular, and ascending deep temporal arteries.

5. The ocular, under which name are included a bunch of arteries arising from the bottom of the orbit, which are distributed to the fatty matter thereabouts, to the muscles of the globe, and to the lachrymal gland, 
eyelids, and ductus ad nasum. One in particular, larger than the others, named the lateral nasal branch, enters the cavity of the cranium through the foramen orbitale internum, and after forming a communication there with the anterior cerebral artery, turns round and enters the nose through the ethmoidal cells to be dispersed upon the Schneiderian membrane.

6. The infra-orbitar, a considerable branch which enters the infra-orbitar canal, in order to supply the anterior molar teeth and medullary substance of the bone with blood: having served this purpose, it sends its remaining twigs out upon the cheek, through the maxillary hole there, where they anastomose with the ramifications of the facial artery.

7. The palato-maxillary, the largest of the terminating divisions of the inferior maxillary artery, enters the foramen palatinum superius, descends through the palatine canal, re-appears upon the roof of the palate, and follows the tract of the palatine groove, some short distance removed from the sides of the molar teeth, and inferiorly makes a curve inward, just above the roots of the front teeth, to take its passage through the foramen incisivum, in front of which it joins its fellow: from this remarkable arterial union issue several branches, some of which run down to supply the glandular structure of the upper lip, while others are directed upward upon the external nares, and the deepseated ones penetrate the dilatator narium and depressor labii superioris; many of them anastomose with the terminating ramifications of the superior labial artery. This artery gives off some few branches to parts at the back of the orbit, before it enters the fora- 
men; and other short twigs, as it courses the palate.Sportsmen and farriers have a practice of cutting through the bars and severing this vessel in order to detract blood on any occasion of emergency : the artery however seldom bleeds much-it soon retracts into its cellular case and forms a coagulum, on which account the operation is, generally speaking, both harmless and ineffectual.

T'he second and smallest division of the external carotid is the

\section{Ramus Anastomoticus.}

IT leaves the trunk of the carotid, commonly at the angle formed by the external and internal carotids, crossing the latter in proceeding to the spine. Deeply seated under the parotid gland, in its course it describes an are backward, which, in the ordinary position of the head, nearly corresponds to the under border of the stylo-maxillaris. From below the coronoid or styloid process, it turns under the transverse process of the atlas, where it joins the vertebral artery, as soon as the latter has passed through the foramen in that bone. From about the middle of the arch comes off

THE OCCIPITAL ARTERY, a vessel nearly equal in magnitude to the trunk itself, which pursues a flexuous course to the occiput, first ascending upon the coronoid process, and then climbing the occipital ridge to reach the vertex. Its branches are-1. A long, slender, deep-seated one, which mounts upward and enters the cranium through the foramen lacerum to be dispersed upon the dura mater: this is sometimes a branch from the anastomotic trunk. 2. Twigs forwarded to the 
temporal muscle. 3. Terminating ramifications to the straight and oblique muscles of the occiput.

The third division of the carotid is the

\section{Internal Carotid Artery.}

THIs vessel, whose calibre is not more than half that of the external carotid, is deeply lodged within the submaxillary space, whence it ascends to the base of the skull, crossing the upper extremity of the cornu of the os hyoides inwardly in its course, which is rendered remarkable by several tortuous turns : it first curves backward, next inward, then upward, and lastly forward, to reach the anterior part of the foramen lacerum, through which it enters the cavity of the cranium. It is accompanied in its course by one of the principal forming branches of the jugular vein, by the eighth pair of nerves, and by the sympathetic. At its entrance into the skull, we find the artery lodged within the cavernous sinus, wherein it makes two more turns, one forward, the other inward, from which last flexure comes off a vessel named the

1. Arteria communicans, which runs in a direction upward and backward, passing under the crura cerebri, to join the basilar, with which it makes one continued vessel, thus forming one side of the circulus arteriosus, to be presently described.-After having given off this vessel, the internal carotid pierces the dura mater, and takes its course upward alongside of the optic nerve, and just over the optic decussation splits into four principal divisions. At the point of division however, or prior to it, are sent off-2. Two or three long branches of small size which run forward 
and spread their ramifications upon the anterior and inferior portions of the dura mater.

The four principal divisions are

1. THE ANTERIOR CEREBRAI, which advances by the side of the optic nerve, and in front of its decussation transmits a considerable branch across, which unites with a similar one coming from the opposite division, thereby forming a vessel nearly equal in size to the internal carotid itself, named the arteria corporis callosi : this is reflected upward and forward around the corpus callosum, and pursues through its entire length the tract of the raphe, detaching as it proceeds numerous lateral twigs for the supply of that body. The anterior cerebral artery now subdivides into several branches, which, with the exception of one, are distributed over the anterior lobes of the brain. This one,

The opthalmic artery, leaves the cranium through the foramen lacerum orbitale; in company with the nerve of the same name, and, at the bottom of the orbit, after forming some anastomoses with the orbital artery, furnishes a twig to the lachrymal gland, long ciliary arteries to the choroid coat and iris, and the central artery of the retina.

2. The MiddLe ARTERIES OF THE CEREBRUM, which come off together at right angles from the trunk, take a flexuous passage between the anterior and middle lobes, and ramify extensively within their substance.

3. ThE INTERNAL ARTERY OF THE CEREBRU, which arises a little higher than the former, pursues the course of the tractus opticus, and winds round to the tubercula quadrigemina. 
The remaining vessels of the brain are derived from the

\section{Vertebral Artery.}

THis artery enters the cranial cavity through the foramen magnum, and makes its appearance upon the cuneiform process of the occipital bone, where, about opposite to the middle of the medulla oblongata, it unites with its fellow, the two forming a single trunk denominated the basilar artery. The vertebral itself gives off-1. Posterior arteries to the dura mater. 2. Ramifications to the medulla oblongata.

The BASILAR ARTERY sends off-

1. The posterior arteries of the cerebelLUM, which leave it as it passes under the medulla oblongata, wind upward round that body, and cross over to the cerebellum, to the posterior portions of which they are distributed.-The basilar artery then continues its course under the tuber annulare, detaching lateral branches as it advances, and next passes between the crura cerebri, where it bifurcates. Its bifurcations proceed but a short way before each of them subdivide into two vessels: one runs forward to meet the communicating artery; the other is the

2. Anterior artery of the cerebellum, which winds outward, around the crura cerebri, thence crosses to the cerebellum, and is principally expended within its lateral lobes.-Below the origin of this artery, from the sides of the arterial circle come off three or four considerable branches, the principal of which is the

Posterior artery of the cerebrum. This 
is directed obliquely backward, across the crus cerebri and tractus opticus, and then mounts upon the posterior lobe of the cerebrum and distributes its ramifications.

The circulus arteriosus, then, is completed anteriorly by the transverse branch of the anterior cerebral divisions ; laterally, by the communicating arteries; and posteriorly, by the bifurcations of the basilar artery. 


\section{LECTURE LXXI.}

\section{The Posterior Aorta,}

\section{Considerably longer and larger than the ante-}

rior, is the main trunk from which are derived the arteries of the abdomen, pelvis, and posterior extremities, in addition to the posterior intercostals and some few of the thoracic arteries. It commences opposite to the fourth dorsal vertebra, at some little distance removed from the spine; from its origin, it curves first upward and then backward, having the pulmonary artery on its left, the termination of the windpipe on its right; having reached the bodies of the dorsal vertebræ, it enters the superior mediastinum, and afterwards takes a straight course along the spine, inclined to the left side, having now the esophagus and vena azygos to the right, the thoracic duct to its left. This portion of the vessel, often distinguished as the THORACIC AORTA, gives rise to many branches, but they are but small.

1. The bronchial arises from the inferior part of the curvature of the trunk, bends its course downward and forward to the root of the left bronchial tube, where it divides into two-the right and left bronchial 
arteries. These vessels penetrate the lungs in company with the bronchiæ, to the branches of which they cling in the course of their ramification within the substance of the parenchyma.

2. THE ESOPHOGEAL also springs from the concavity of the arch, near to the former, sometimes before it, and then proceeds backward and to the right, to reach the esophagus. Here it splits into a superior and inferior artery, which course the entire length of the tube, distributing branches right and left over its surface, and terminating at the cardiac orifice in anastomosis with the gastric artery.

3. The intercostal, the remaining branches, come off in pairs from the sides of the vessel, to supply all those intercostal spaces posteriorly to the last, which received a branch from the anterior intercostal artery. These arteries preserve the lines of the ribs, running close to their posterior borders, and end about the inferior parts of the chest and abdomen in anastomosis with the internal pectoral and epigastric arteries. They furnish, near their origin, small branches which enter the vertebral canal through the spinal holes, and also numerous muscular twigs during their course.

Having detached these small vessels, the posterior aorta continues its passage between the crura of the diaphragm into the abdomen : in making its exit from the chest however it gives rise to two small arteries, or to a single branch which afterwards forms two, named the

Phrenjc or Diaphragmatic arteries, right and left. These vessels penetrate their respective crura, in whose substance they ramify, and nltimately disappear upon the cordiform tendon. Within the abdo- 
men, the aorta continues to be firmly fixed to the spine by means of its several vessels and cellular attachments, and thus proceeds, still inclined to the left side, as far as the last lumbar vertebra, under the body of which it splits into four large arterial trunks. Prior to this division, the ABDOMINAL AORTA gives off-

1. THE CELIAC ARTERY, which is nothing more than the common root of the splenic, gastric, and hepatic-arteries that in some instances have separate origins: it is springing from the under part of the trunk, a little posteriorly to the origin of the phrenic.

a. The splenic artery, the middlemost of the divisions of the cœliac, takes a winding course to the left side of the cavity, turns forward along the greater curvature of the stomach, running between it and the concave part of the spleen, and at length ends in the left gastric artery. Soon after its origin, it gives two or three pancreatic arteries as it passes that gland. Many considerable branches to the spleen from the convexity of its flexure; whilst, from the concavity of it are passing smaller but longer branches upon the greater curvature of the stomach. It is prolonged beyond the tapering termination of the spleen, distributing shorter branches to the stomach as it proceeds, and continuing to encircle the organ towards its right extremity, from which is coming to inosculate with it, the right gastric artery.

b. The gastric artery, the smallest of the cœliac divisions, runs forward to the small curvature of the stomach, between the layers of the omentum, splitting before it reaches the organ into two branches that take the names of superior and inferior gastric: they spread their ramifications, in an arborescent form, upon the up- 
per and under surfaces of the stomach, and they anastomose with those of the right and left gistric.

c. The hepatic artery, the largest of the cocliac divisions, proceeds before the pancreas to the right side of the cavity, and winds forward over the pyloric end of the stomach. It gives off-1. Divers small branches to the pancreas, as it passes by the gland. 2. Near the pylorus, it sends a considerable branch to the beginning of the duodenum, which, as soon as it reaches the intestine, bifurcates: one division, the duodenal, retrogrades along the gut, and ends in anastomosis with branches coming from the anterior mesenteric; the other, the right gastric, crossing the gut, proceeds to the great curvature of the stomach, where it inosculates with the left gastric. The hepatic artery itself is continued forward to the porta of the liver, where it divides into the right and left hepatic: the right, the larger and shorter one, after giving off a considerable branch to the portio media, turns back to reach the right lobe; the left also gives off a branch or two to the middle portion, but they are but small, and then runs to the left, along the left fissure, to penetrate the other lobe.

2. TIE ANTERIOR OF GREAT MESENTERIC is the next vessel to the coliac, a little behind which it is arising from the under part of the posterior aorta. The trunk of this artery is extremely short; but it is of large size-aneurismal indeed in the ass species, and is the parent stock of a numerous collection of extensive. ramifications. From its origin it passes directly down-ward within the layers of the mesentery, detaching from behind some slender twigs to the pancreas in its descent, and then suddenly separates into a set of larger branches, (commonly from eight to twelve in 
number) varying in length and size, from which are derived-a. A branch that runs to the duodenum, within the concavity of which it divides into two small ones, which turn in opposite directions and form arches, one with the duodenal artery, the other with the next mesenteric, from the convexities of which arise other small transverse vessels, which encircle the intestine and ramify to great minuteness under its peritoneal covering, forming altogether a beautiful vascular network. b. A collection of branches of considerable length, more uniform than the rest, run backward for the supply of the other small guts: these in like manner run in arches and inosculate, and furnish an abundant supply of nutrient and secerning vessels. c. 'Two branches, shorter than the others, proceed to the cœcum caput coli, whence they run along the sides of the cœecum, sending off numerous transverse branches which encircle the gut and anastomose freely with each other. $d$. Several long and short branches to the colon, which furnish with lateral ramifications, distributed after the same manner, the cœcal portion of the gut, and its two great flexures or arches: the last of these branches, which is directed towards the sigmoid flexure, is met in anastomosis by the first of the posterior mesenteric.

3. ThE RENAL or EMULGENT ARTERIES leave the aorta at right angles, one on each side, either immediately opposite or somewhat posteriorly to the origin of the preceding vessel. They pass in straight lines directly outward, and each enters the notch of its appropriate kidney, and therein divides into three or four large branches which penetrate the glandular substance. The right is the longer one, in consequence of 
the inclination of the aorta to the left side and its having to cross the vena cava posterior in its course. It furnishes in its way- $a$. Twigs to the enveloping adipose membrane. $b$. A small artery destined for the supply of the capsula renalis: in some instances this last vessel comes from the aorta ; rarely, from the anterior mesenteric.

4. The spermatic arteries, right and lefi, in comparison to their diameter, are the longest in the body, springing near to each other from the under part of the aorta, about midway between the origin of the renal arteries and its bifurcation: in some instances these vessels come from the posterior mesenteric. They pursue divergent and flexuous courses backward, pass out of the abdomen at the internal abdominal rings, and there enter into the constitution of the cords with which they proceed to the testicles. In the female, these arteries run within the layers of the broad ligaments to the ovaries to which they are principally distributed; but they also transmit branches to the Fallopian tubes and to the horns of the uterus.

5. The Posterior or SMall mesenteric, the last of the abominal arteries, a much longer vessel than the anterior mesenteric, comes from the under part of the aorta, commonly very near to the roots of the spermatic arteries. It descends within the folds of the mesocolon, and distributes its principal branches to the left - to the sigmoid flexure of the colon; the others run backward and ramify upon the anterior portion of the rectum: their arrangement and distribution are similar to the colic ramifications of the anterior mesenteric. The anterior division anastomoses with the pos- 
terior of the great mesenteric; and the posterior forms communications with the other arteries supplying the rectum.

6. Five or SIX PAIRS OF LUMBAR ARTERIES, according to the number of vertebra, are also furnished by the posterior aorta. Arising in pairs from its sides they wind upward, covered by the psoas parvus, follow the inter-vertebral spaces, pierce the fibres of the intertransversales lumborum, and ramify and expend their branches among the muscles covering the loins. In the course of their ramifications they contract communications with the last of the intercostals and circumflex of the ileum. Near their origins, each detaches a branch which enters the vertebral canal by the correspondent spinal foramen, also twigs to the psoas parvus and intertransversales.

Under the last lumbar vertebra the aorta bifurcates into two pairs of large and important arterial trunks :the external and internal iliacs.

\section{The Internal Iliac Arteries}

Arise by a kind of second bifurcation from the trunk; for there is still a continuation of the aorta after it has given off the external iliacs; and this forms a remarkable difference between it and the division of the corresponding vessel in the human subject, the horse having no common iliac arteries. The internal iliacs, though larger than the extemal, are extremely short; they diverge from the parent trunk backward and outward, and hardly quit the body of the vertebra before each gives off a large branch, the artery of the bulb; it then runs a little further, and by the side of 
the sacral articulation splits into three nearly equal divisions:- -the obturator, gluteal, and lateral sacral arteries.

THE ARTERY OF THE BULB, I repeat, springs from the under and outer part of the internal iliac, and then runs backward along its outer side, where we find it placed between the trunk and the external iliac vein, which latter at the same time is a little above it. It makes a sweep backward, around the sides of the pelvis, to which it is closely bound by peritoneum and cellular adhesions, then insinuates itself between the laminæ of the sacro-sciatic ligament, and continues along the upper border of the ischium to the ischial arch, the side of which it turns round to reach the bulb of the penis, wherein it terminates. In the female, this artery sends its terminating branches to the bulb of the vagina and lining membrane of that canal. It gives off-1. The umbilical artery, or rather the remains of that vessel, which in the foetus nearly equalled in size the iliac arteries themselves, and was surpassed by none in regard of importance. In the young subject these vessels commonly remain pervious as far as the bladder; but in process of time they degenerate into the round ligaments of the bladder; in the description of which organ notice has already been taken of them. 2. Sundry small vesical branches, in passing, to the bladder. 3. In leaving the pelvis, the prostatic artery, which detaches twigs to the vesiculæ seminales, but distributes its ultimate ramifications to the prostrates. 4. Divers branches, anal and perineal, to the posterior portion of the rectum, anus, and parts composing the perineum. 
THE obTuRator ARTERY, the lowermost of the divisions of the internal iliac, corresponds in the first part of its course to the brim of the pelvis, in which it is accompanied by the crural nerve; it subsequently inclines upward to gain the posterior nook of the foramen obturatorium vel magnum, where it runs through the perforation in the obturator ligament. It then turns round the branch of the ischium, and in front of the bone ends in division into the ischiatic, pubic, and internal pudic arteries. Its branches are-1. The arteria innominata, one of very large dimensions which comes off not far from its origin from the iliac. This proceeds backward and outward, close under the pelvic margin, crossing obliquely the external iliac artery and the psoæ and iliacus muscles, and dips deeply into the substance of the haunch, wherein it sends branches downward to the rectus, upward to the tensor vaginæ. 2. Ramifications to the obturator muscles and ligament, in passing through the foramen. Its divisions are1. The ischiatic, which turns down upon the back of the haunch, passing opposite to the hip-joint, and distributes its branches to the triceps. 2. The pubic runs backward along the branch of the ischium, and is expended in the large head of that muscle. 3. The internal pudic artery turns inward and backward round the ischial arch to the root of the penis, where it splits into two sets of branches. a. One set penetrate and end in the crus penis. $b$. Two or three branches belonging to the other set run further forward and pierce the fibrous case of the corpus cavernosum. $c$. One or two slender branches of the same run along the dorsum penis. $d$. Another accompanies the pudic 
nerve to the extremity of the organ. $e$. Besides which there are sundry cutaneous twigs.

The gLUTEAl a RTERY, the middlemost of the iliac divisions, shortly after its origin leaves the pelvis through the hole in the sacro-sciatic ligament, at the anterior nook of the notch, in company with the sciatic nerve, which runs behind it: immediately that it has made its exit, it splits into two or three branches of large size whose ramifications are destined principally for the gluteal muscles, though some descend to aid in the supply of the posterior femoral muscles.

THE LATERAL SACRAL ARTERY runs directly backward, following the sides of the sacrum, to which being closely bound by cellular adhesion it necessarily takes the slight curve of that bone: having reached the coccyx it splits into two long, slender, terminating branches. It furnishes-1. Sacro-spinal branches, five or six in number, which enter the spinal canal through the internal sacral foramina. 2. The peroneal artery, a branch as large as, or even larger than, the trunk itself, of which it might be more correctly perhaps considered as the continuation. It soon divides into several ramifications, of which-a. many run to the gluteal muscles; $b$. while others descend upon the back of the thigh; $c$. and a third set are distributed to the anal muscles, and to the skin and cellular substance of the perineum. 3. The lateral coccygeal artery, one of the terminating branches of the lateral sacral, runs close to the side of the os coccygis, even to its extreme point, preserving the line of direction of the trunk, diminishing in diameter as it recedes, and distributing numerous branches laterally to 
the coccygeal muscles. 4. The inferior coccygeal artery, rather larger than the lateral, takes the same course along the inferior and lateral part of the bone, and may also be traced to the tip. It sends down a long slender twig or two to the anus; the remainder of its branches are distributed to the coccygeal muscles.

THE MIDDLE SACRAL ARTERY is a very inconsiderable branch coming off, generally, from the trunk at its angle of bifurcation into the internal iliacs. It proceeds a little way backward upon the middle of the sacrum, and then vanishes.

\section{The External Iliac Artery,}

Right and left, results from (what may be significantly described as) the first bifurcation of the posterior aorta, which takes place underneath the body of the last of the lumbar vertebræ. It takes the same oblique direction (outward and backward) as the internal iliac, but it is less in diameter, and bears no comparison in respect to length. The best guide to its situation is the brim of the pelvis; considering the bowels to be removed, it runs along this brink of the cavity, uncovered by any thing but peritoneum, following the bony curvature outward to its termination, which we mark upon the same brim about midway between the symphysis pubis and anterior spinous process of the pelvis, from which place it plunges into the prominent mass of muscle forming the inside of the thighs. The vessel gives off -

1. The CiRCUMFLE ARTERy OF ThE ILEUM, a large branch that departs from the outward side of the trunk, near its origin. It winds directly across the loins 
to the flank, covered only by peritoneum, crossing in its way the two psoæ and the iliacus; ariving in front of the anterior spinous process of the os innominatum, it splits into two ressels, which take opposite courses: one inclines backward, penetrating the transversalis, to which and to the originating portion of the internal oblique muscle its ramifications are distributed; the anterior and longer division curves forward between the transverse and internal oblique muscles, and sends its ramifications to them, but principally to the latter, with the exception of its ulterior twigs, and they run on and form communications with the last intercostals. These vessels also anastomose, among the muscles, with the lumbar arteries.

2. THE ARTERY OF THE CORD, which in other cases comes from the last-described branch, or from the aorta itself, arises from the under part of the vessel, a little further backward than the circumflex. This is a very slender vessel, but of sufficient length to run obliquely back ward to join the spermatic cord and proceed with it through the inguinal canal, for the general supply of the parts entering into its constitution.

3. THE ARTERIA PROFUnda FEMoris is a very large branch which may be said at its origin to mark the extent of the iliac artery posteriorly and the femoral anteriorly, or one that may be considered to be given off by either of the trunks at the place where they individually change their names. The profunda plunges at once into the thick of the haunch, and then makes an oblique curve round the neck of the os femoris, passing under the short and long heads of the triceps. Having reached the posterior quarters, it sends its ra- 
mifications principally into the biceps. Before this vessel dips into the substance of the thigh it gives rise to a large branch,

The epigastric artery, which at first makes a curve backward, downward, and outward, in doing which it turns round the inward margin of the internal ring, running at the time between the peritoneum and the tendon of the transversalis; next, it makes a sweep outward and subsequently upward, in the course of which it gradually insinuates itself between the disgregated tendinous fasciculi of that muscle until it at length is found pursuing its way between the transverse tendon and internal oblique muscle. It then continues its passage forward, within the sheath of the rectus, along the upper and inner border of that muscle, furnishing as it goes ramifications from its sides, and at length ends in anastomosis with some ramifications coming in a contrary direction from the internal pectoral. In passing the ring, it gives origin to a considerable branch which turns round the crescentic borders of the tendons forming that aperture and then splits into several small arteries. Of these- $a$. A twig runs to the groin and ramifies among the adipose membrane and absorbent glands there. $b$. A long slender branch makes its way to the ring and descends upon the cremaster; this is furnished however in some instances by the external iliac. $c$. A subcutaneous twig to the thigh. $d$. The external pudic, the largest and longest of these divisions, when this is their arrangement, for the origin of these vessels is attended with much variety: it runs forward, invested in the subcutaneous cellular substance of the scrotum, sending ramifications to the tunica va- 
ginalis, and the skin, and continuing forwarditupon the penis, where it inosculates with the superficial ramifications of the internal pudic.

\section{The Femoral Artery.}

REGARDING the profunda femoris as a limb of the external iliac, we descend to the femoral artery, the subsequent continuation of the same trunk. This artery proceeds in an oblique direction down the haunch, preserving nearly the line of its middle; it might appear from its origin, and from no part of it being brought into view by the removal of the faschia, that it directly plunged deep among the muscles; this however is by no means the case, for the upper half of the vessel may be considered, in an anatomical sense, as superficial: an incision carried along the prominent anterior border of the gracilis, separating it from the sartorius and pushing the latter forward, at any point in the upper half of the thigh, will immediately expose the vessel. The beginning of the trunk, which is curved outward, is covered by lymphatic glands of the groin; it is then overlapped by the thin posterior edge of the sartorius, and subsequently by the anterior border of the gracilis, running rather below the middle of the thigh within a triangular space formed by the approximation of the two; here the vessel dips deeply into the muscular substance, having the long head of the triceps on its inner part, the bone on its outer, and afterwards takes its course between the heads of the gastrocnemius externus into the hollow at the back of the stifle, wherein we find it close to the joint, but nearer to the outer than the inner side: opposite to the 
head of the tibia it bifurcates into the anterior and posterior tibial arteries. The femoral trunk is accompanied by its vein, which is placed at first behind and then gets to the outer side of it; and the principal branch of the crural nerve runs for nearly one-third of the way along the outer side of it also: they are all three invested in some loose cellular substance. The anterior branches are-1. The inguinal, one of large size arising in the groin, covered at its origin by the lymphatic glands, to which it sends twigs; it then crosses under the sartorius, to which it also sends ramifications, and gets between the vastus internus and rectus, where it splits into two divisions which penetrate those muscles. 2. Three or four small branches to the sartorius. 3. A long slender one to the side and front of the stifle. Its posterior branches are-1. A considerable one to the gracilis, which detaches twigs to the long and short heads of the triceps. 2. A small one that turns inwardly to the vastus internus. 3. A branch supplying both the long and large heads of the triceps. 4. One that turns round the back of the bone about the middle of the thigh for the supply of the biceps. 5. One large or two smaller branches sent along the posterior border of the gastrocnemius externus, from which recurrent ramifications ascend to the triceps. At the back of the stifle come oft the popliteal branches, four or five in number, taking opposite directions, which are destined for the supply of the joint: one ruus down upon the posterior tibial muscles; another, the recurrent branch, climbs the back of the os femoris and anastomoses with the descending ramifications of the profunda femoris. 


\section{The Tibial Arteries,}

Anterior and posterior, are to be regarded in no othei light than the bifurcated continuation of the femoral trunk : this division, I before remarked, happens at the back of the head of the tibia.

The posterior tibial antery, the smaller of the two, is curved outward at its origin; it then descends along the posterior deep region of the thigh, inclining all the way from the outer to the inner side, at first between the flexor pedis and the popliteus, which is behind it, subsequently between the former muscle and the flexor pedis accessorius, and at length between the tendon of the last-named muscle and the inner, posterior, and inferior part of the body of the tibia. Just above the hock, it inclines inward again and gets deep-seated between the lower end of the flexor pedis and the bone, where it ends in bifurcation. Its branches are-1. One, which comes off a short distance from its origin and runs into the flexor pedis. 2. The medullary, which enters the medullary foramen in the upper and back part of the tibia. 3. Unimportant twigs to both the flexors. Of its terminating branches-the external one proceeds round the outside of the hock, ramifying there subcutaneously and anastomosing with some articular twigs of the anterior tibial; the internal continues down the leg over the tendon of the flexor pedis, within a cellular sheath formed between that tendon and the root of the os calcis, in company with the internal metatarsal nerve, and creeps along the inner edge of the metatarsal bone, between the flexor tendons and suspensary ligament, 
ending at the lower part of the cannon in divers small ramifications. At the hock, this artery sends off one or two recurrent branches which ascend upon the root and back of the os calcis, and anastomose with others coming down from the posterior tibial.

THE ANTERIOR TIBIAL ARTERY no sooner leaves the common trunk than it suddenly turns forward and passes between the tibia and fibula to the fore part of the thigh; here we find it between two veins, crossing with very gradual obliquity the outer part of the bone to gain the front, which it does about midway between the stifle and hock; it then continues to descend between the outer border of the flexor metatarsi and the bone until it reaches the front of the hock; here it makes another sweep outward, and ultimately arrives in the channel between the external and large metatarsal bones, where it becomes the metatarsal artery. Its branches are-1. Some small recurrent articular, which ascend to the stifle and communicate with the popliteal branches. 2. Various muscular branches, in its course down the thigh. 3. Divers, small, articular and cutaneous branches, as it obliquely winds from the front of the hock to its outer and anterior part. 4. A slender metatarsal artery which descends upon the front of the cannon, adhering to the bone, along the inner edge of the extensor tendon, whose ramifications, mostly cutaneous, are distributed over the inner and fore part of the leg, the terminating ones reaching as low as the fetlock.

The METATARSAL a RTERY pursues its course, unaccompanied by any vein, along the above-noticed channel for two-thirds or thereabouts of the length of the 
leg; it then passes between the internal and large metatarsal bones, and gains the posterior part of the latter, between which and the suspensary ligament, a little above the fetlock, it divides into three vessels: one forms an arch (as in the fore extremity) from which come off the recurrents, and they anostomose with the posterior tibial artery; the lateral divisions become the plantar arteries.

Henceforward, the arteries correspond in the fore and hind extremities; one description therefore suffices for both. 


\section{LECTURE LXXII.}

\section{Distribution of Veins.}

THE veins over the body generally have a similar arrangement and distribution to the arteries, though they are perhaps at least double the number of those. vessels: where we find an artery, we almost invariably find a vein, and very frequently meet with two veins; besides which, there are many veins that have no arteries accompanying them. In our descriptions of the arteries, every now and then, we met with some notable variations from the ordinary or general distribution; but the veins vary greatly more in their course and division into branches than the arteries, inasmuch, indeed, that any description of them in one subject, however accurately drawn in that instance, will never hardly be found correct in every particular when applied to another. Like the arteries, they are arranged in two general systems : the one, consisting of the pulmonary veins, exclusively belonging to the lungs, has been already described; the other, distributed over the body generally, forms the subject of our present inquiry. With this last is connected a peculiar venous arrangement subservient to the liver, which is often 
The Anterior Vena Cava.-The Jugular Vein. 355 made a distinct consideration of, under the title of the system of the vena porta.

The two main venous trunks, THE VENÆ CAVE, anterior et posterior, correspond to the anterior and posterior aortæ; the one is the main returning venous conduit belonging to the fore parts of the body, the other that into which the veins ultimately run coming from the hind parts: these trunks, with their various ramifications, consequently, are totally unconnected, one with the other, and this circumstance furnishes us with a convenient division of the whole into the vessels of the anterior and those of the posterior vena cava.

\section{The Anterior Vena Cava}

Forms the main or common trunk of the veins returning the blood from the head, neck, parietes of the chest, and fore extremities. It is a short vessel, but one of considerable volume, ending by a bulky termination in the summit of the right auricle, having the trachea above, and the anterior aorta to its right, and with both included between the layers of the mediastinum. The anterior cava is principally formed by the concurrent union of the jugular and axillary veins, and is situated at its formation in the space between the two first ribs, about midway between the sternum and vertebræ; it also receives the pectoral, vertelral, dorso-cervical, and inferior cervical veins, and the vena azygos, which augment its volume in its course to the heart.

\section{The Jugular Vein.}

THis forms the principal venous conduit from the head and neck, corresponding in course and ramifica- 
tion to the carotid; we have therefore a right and a left jugular vein, and it is quite unimportant which we take by way of description. The jugular vein takes its rise at the foramen lacerum basis cranii, from the termination there of the lateral sinus of the dura mater, from which it receives the blood returned principally from the cerebrum, partly from the cerebellum. Concealed at its origin by the condyle of the jaw, it descends to the inward side and behind the neck of the condyle, deeply buried under the parotid gland; lower down, it makes its appearance behind the branch of the jaw, and there joins the external carotid artery, along with which it continues its course into the neck. In this part of its course it receives the following branches of importance-1. THE AURICULAR VEINS, anterior and posterior, and also internal, varying in their number and mode of termination, which are seen descending over the root of the ear. 2. THE TEMPoRAL, a vein of considerable size, taking its course along the upper side of the temporal artery. 3. THE INTERNAL MAXILLARY, a large vein found in company with its artery: in its course it receives the blood of many small veins-the palato-maxillary, infira and supra orbitar, ocular, inferior maxillary, and deep temporal. 4. THE PAROTIDEAL, numerous veins from the parotid, and some also from the submaxillary gland. 5. Branches from the masseter and pterygoid muscles.

6. The occipital vein, a long flexuous branch descending from the head along with the occipital artery. It brings blood from the occipital sinuses, receives veins from the posterior lobes of the cerebrum, and from the cerebellum; also veins from the dura 
mater, and several muscular branches in the course of its descent.

7. Thl submaxillary VEI, the most considerable branch of the jugular, so large indeed compared to the continued trunk that some regard the two together as a bifurcation of the common jugular. It is formed upon the side of the face by the concurrence of the facial, Inbial, and varicose veins, whence it turns round the branch of the jaw, between the artery of the same name and the parotid duct, and joins the trunk by the side of the trachea, just below the parotid wland. In its course it receives other veins which likewise contribute to its volume, of which the principal are-the submental, sublingual, lingual, pharyngeal, and superior laryngeal veins. The facial vein results froma ramous expansion of small veins upon the side of the face, receiving one or two branches, one of which is commonly varicose, from the masseter. The labial vein is formed by the union of a plexus of venous branches coming principally from the angle of the mouth, joined by others both from the upper and lower lips. The varicose vein is a vessel deeply buried in the substance of the masseter, descending obliquely along the furrow made by the approximation of the jaws when the mouth is shut, and is one remarkable for having naturally a large varix or reservoir for blood at its origin: not however that this is the only varicose vein, for the temporal commonly bulges more or less, as well as a vein from this muscle already noticed; but this is the largest specimen of so remarkable a formation. I believe that these varices serve to guard against stagnation or congestion in the brain and its membranes, and from 
consequent rupture of their sinuses and delicate veins, by receiving the blood at the time that the circulation is interrupted or impeded from the motions of the jaw.

The jugular trunk having received the submaxillary vein in the space between the larynx and vertebra dentata, proceeds down the side of the neck, covered by the cervical portion of the panniculus carnosus, within the channel formed by the lower border of the levator humeri and the upper one of the mylo-hyoideus; by a layer of oblique fibres coming from the former of which muscles it is separated from the carotid artery and trachea, which run to its inward side and rather below it ; in its course down the lower half of the neck however it gets deeper-seated, approaches the windpipe, and afterwards runs in company with the carotid artery. It terminates in the anterior vena cava within the space between the two first ribs. At or near the junction of the submaxillary, the jugular vein receives some small veins, principally thyroideal; during its course it also receives many small branches-cutaneous, muscular, and tracheal reins. Near its termination it receives a considerable branch, the superficial brachial vein, which arises in front of the radius, about twothirds of the length of the bone upward, from the main brachial vein, and ascends along the anterior border of the biceps, and continues upward in the hollow between the breast and the arm, winding as it proceeds a little outward, to reach the jugular: this by farriers is called the plat-vein-it is the one we are in the practice of opening for lameness in the shoulder. It receives some unimportant muscular and cutaneous branches in the course of its ascent. 


\section{The Vertebral Vein}

Is the fellow vessel to the vertebral artery, taking the same course, on either side of the neck, from the head to the chest, through all the foramina in the transverse processes of the cervical vertebræ with the exception of the last. This vein has conmunications with the occipital sinus and posterior cerebral veins, but it uwes its formation principally to the veins coming from the medulla oblongata, and the spinal marrow and its membranes: it also receives vessels from the deepseated muscles in the vicinity. It takes its course along the upper side of the vertebral artery, and ends in the anterior vena cava, just behind the first rib.

\section{The Axillary Vein}

Forms the main channel for the return of the blood distributed by the divisions and ramifications of the axillary artery (to which it corresponds) to the various parts of the fore extremity. Its forming branches may be arranged into sets, a superficial and a deep-seated set: the former run for the most part immediatcly underneath the skin; the latter, exceeding them in number and size, accompany the arteries among the muscles; we meet however with divers vessels of communication between them in their course. It will facilitate the description of these vessels, and be the most natural mode of proceeding, for us to descend at once to the foot and trace them from their origin; I shall therefore first direct my attention to the

Plantar vesns. There is not perhaps a piece of vascular structure in any part of any animal that can be exhibited as a specimen of greater beauty of venous 
arrangement than the foot of the horse: the sole displays a curious and intricate network of small veins, and the laminæ in every part show the same reticular venous ramification, altogether giving the foot really quite a covering of venous netting. We have seen that the arteries of the foot elude pressure, and consequent impeded or interrupted circulation, by running through the interior of the os pedis; we now perceive that the veins, running all outside, (for, to have admitted them also through it, the bone must have been perforated in so many places that it would have been inadequate to the superincumbent burden,) guard against the impediments necessarily occasioned to the circulation in some of them by the numbers of communicating canals which are provided in every direction: in addition to which, we may recall to mind, that they have no valves.

The veins of the sole pour their blood into the veins of the laminæ, with the exception of some few of the posterior most, and they end in the veins of the frog.

The veins of the lamina increase in size towards the coronet, and gradually unravel themselves so as to collect into a great many plexuses or bunches; these run directly upward, crowd through the substance of the coronary ligament, and afterwards form the superficinl coronary vein. From them, larger branches proceed laterally which are again joined in communication by the deep coronary, a vein commonly double; after which, the larger veins upon the sides diminish in number, and all conjoin into two or three branches which, opposite to the pastern-joint, unite into a single vein.

The veins of the frog, after ramifying in the form 
of network over that body, ascend along the inward sides of the cartilages into the heel, converging and growing larger as they leave the foot, and making a single branch at the pastern-joint, which runs up and unites, a little above the head of the os suffraginis, with that coming from the laminæ, thereby forming the plantar vein.

The plantar vein in its ascent preserves the line in front of the plantar artery, accompanying that vessel till they both unite with their fellow trunks of the op posite side and become metacarpal. In its course, it is joined by the perpendicular vein, besides other small unimportant branches: at its termination, it reeeives one or two veins of considerable size which emerge from the fetlock-joint.

The metacarpal veins, two in number, result from the union of the plantar, which takes place just over the tops of the sesamoids, in the channel between the flexor tendons and the suspensary ligament. These veins pursue their course up the leg, one on either side, along the same channel between the tendons and the ligament, to the back of the knee, where they end in a remarkable anastomosis, which is formed by a transverse branch equal in diameter to either of the trunks. The internal metacarpal vein accompanies tho artery, having that vessel behind and the inner small metacarpal bone in front; the external vein, the smaller and deeper-seated one, also preserves the line of the splint bone on the other side, but it is more inwardly placed. These vessels receive in their course cutaneous veins from the front of the cannon, and at their origin one or two descending veins from the back of the $\mathrm{lcg}$. There is some variety commonly found in regard to the 
number and disposition of the anastomosing and other branches at the back of the knee; but there are ordinarily two principal venous trunks, one of which ascends over the inner and back part of the joint, having the artery behind it, the other mounts close to the inward border of the trapezium. As they pass the joint, these vessels receive the blood from the anterior and posterior articular veins. A little above this, they communicate by a short and free anastomosis, and afterwards run on to form the superficial and deepseated veins of the arm.

THE SUPERFICIAL BRACHIAL VEIN, the cephalic of the human subject, the plat-vein of our farricrs, continues to ascend along the inner side of the radius, inclining very gradually forward, until it has arrived at the elbow-joint; here, it crosses obliquely over to the front of the biceps and pursues its ascent upon that muscle towards the point of the shoulder, to the inmer part of which it creeps round, and then plunges inward to reach the jugular vein, in which it terminates. This is one of the superficial veins of the arm, it being covered only by the skin from its origin to its reaching the shoulder-joint; and is, as I observed before, the vein we commonly let blood from. In its course, it receives divers cutaneous and muscular branches which contribute to augment its volume a little; and besides, contracts anastomoses with the other veins of the arm, among which should not be overlooked a large and remarkable communication it has with the humeral vein, just before it leaves the radius, through the medium of a branch which runs directly upward.

THE RADIAL VEIAS, arising from the junction of 
the metacarpal veins above the knee, two in number, take the course of the radial artery, and a little above the shoulder-joint concur with the anastomotic branch coming from the superficial brachial to form the humeral vein. They receive many muscular branches as they ascend, and also anastomosing vessels from the ulnar and superficial reins.

THE ULNAR VEINS are divers small vessels taking the course of their respective arteries. One of them, apart from the others, is connected with the humeral by a separate canal, and makes a curve upon the inward part of the elbow-joint towards the olecranon, and afterwards runs down over the side of that projection along the backs of the flexors, where it becomes superficial, to its termination at the knee. The others all end by a common trunk in the humeral vein, about its middle.

TIE HUMERAL VEIN, the production of the union of the anastomotic branch from the superficial brachial with the radial veins, is a large vessel extending along the inner side of the humerus, in contact with the bone. The humeral artery, which accompanies this vessel, is at first concealed between the bone and the vein, but higher up the artery gets in front of the vein. It receives small veins from the joint, and divers others from the muscles around, whose number is indeterminable, and whose course is best learnt by a reference to that of the arteries. It receives a considerable branch from the triceps.

THE AXILLARY VEIN is the continuation of the humeral, augmented by the accession of the triceps vein. The axillary artery joins the limb directly opposite to the shoulder-joint, and the vein runs imme- 
diately below and alongside of it. It follows the future course of the artery, turning with it round the first rib, to join the vena cava anterior, which it does at the place where that vessel receives the jugular. Its branches are-1. The subscapular vein, one of considerable size accompanying the subscapular artery, receives as it runs along branches from the triceps and. subscapularis, corresponding to the arteries, and either joins the root of this vein or the termination of the humeral. 2. The dorsalis scapulce follows the course of the artery of that name, bringing blood from the antea. and postea spinati, and terminates about midway between the chest and shoulder. The remaining branches of this vein come from the parietes of the thorax. They are-a. The humeral thoracic, comprising two or three veins, corresponding to the arteries so called, the original ramifications of which come from the muscles about the point of the shoulder. b. The external thoracic is a long vein traversing the lower part of the chest, in a horizontal direction behind the arm. A great portion of it runs subcutaneously, and is distinctly traceable in the living animal through the skin, from which circumstance it has been noticed by horsemen and named from its situation the spur vein. It is originally formed upon the abdominal parietes by the union of two convergent branches, which are derived from the integuments of the sheath, or, in the female, the mamma: it receives also, as it crosses over the ribs, other small veins which likewise contribute more or less to its volume.

\section{The Pectoral Vein}

Answers in course and distribution to the pectoral 
The Dorso-Cervical \& Inferior C'ervical Veins. 365,

artery. It originates in branches from the abdominal parietes, continues to receive laterally accessory vessels in its course, and ascends along the inner and posterior border of the first rib, in front of the artery, to reach the lower side of the vena cava anterior.

\section{The Dorso-Cervical Vein}

Consists of two divisions ramifying with the dorsal and posterior cervical arteries, and bringing blood from the muscles which they supply. It also rcceives the anterior intercostal vein, the companion of the artery so named, which is the common trunk of the veins belonging to the second, third, fourth, and fifth intercostal arteries.

\section{The Inferior Cervical Vein}

Is the vessel or vessels running down the inferior part of the neck in company with the inferior cervical artery. The principal branches are muscular, though some come from the skin and absorbent glands in the vicinity.

\section{The Vena Azygos.}

${ }^{\mathrm{T}} \mathrm{TH}$ e last and only single branch of the vena cava anterior, in the upper and back part of which it ends, just as the trunk opens into the auricle. It arises, as far backward as the loins, by some small straggling veins, and takes its course across the bodies of the dorsal vertebræ, on the right side of the posterior aorta, growing somewhat larger as it advances towards its termination, where it forms a remarkable curvature downward, to reach the trunk. It receives at its origin many veins from the neighbouring muscles, those of 


\section{The Vena Azygos.-The Posterior Vena C'ava.}

the loins in particular; but its destined office is to bring the blood from the posterior intercostal veinsfrom 12 to 13 of them on the right side, from 8 to 9 only on the left, in consequence of its being placed itself inconveniently for the reception of more of the latter : had the vena cava posterior been placed alongside of the posterior aorta, a vena azygos would not have been required; it may be said, therefore, to supply the want of that vessel along the spine.

\section{The Posterior Vena Cava.}

THIS is the correspondent venous trunk to the posterior aorta, returning the blood from the parietes of the abdomen and pelvis, the urinary and genital organs, and the posterior extremities. The internal and external iliac veins, unlike their accompanying arteries, join and form two common iliac trunks, from the union of which, upon the last vertebra of the loins, results the main trunk now under consideration. Thus formed, it takes its course under the bodies of the lumbar vertebræ, runs along the great fissure of the liver, perforates the cordiform tendon, and pursues its way directly across the cavity of the chest to the posterior and inferior part of the right auricle. In its passage it is joined by the lumbar, spermatic, renal, hepalic, and diaphragmatic veins.

THE LUMBAR VEINS, correspondent in number and course to the lumbar arteries, result from the union of small veins coming from the muscles clothing the loins; they receive in their subsequent passage, each of them, a branch emerging from the vertebral canal, and afterwards join the main trunk at right angles.

THE SPERMATIC TEIN ascends in company with 
its artery, runs forward with it within the abdomen, and terminates in the under side of the vena cava posterior, occasionally uniting first with its fellow into one common vessel.

The renal or emulgent veins, formed by branches emerging from the pelvis of the kidney, which commonly exceed in number the divisions of the artery, accompanies the renal artery to the spine, and ends in the under and outer part of the main trunk. The left vein is longer than the right in consequence of having to cross the aorta. They receive the veins belonging to the capsulæ renales.

THE HEPATIC VEINS, many in number, spring from the terminations of the vena portæ and hepatic artery, pervade the parenchyma of the liver, and end in the vena cava, as it runs along the great fissure, by numerous orifices resembling pinholes.

The Phrenic or diapilragmatic veins. From the arborescent venous display upon the surface of the diaphragm ascend six branches of considerable size; these however conjoin afterwards and make but two on each side, which enter the trunk as it perforates the tendinous substance.

\section{The Common Iliac Veins}

ARE formed under the sacro-iliac symphysis by the union of the external and internal iliacs. Their course, which is very short, is obliquely forward under the last vertebra of the loins, where they unite and constitute the main trunk. They receive however-1. A considerable vein resulting from the union of small branches coming from the psoæ and iliacus. 2. The circumflex vein of the ileum, from ramifying with its artery. 3. 
At their junction, the middle sacral vein, an azygos vessel of small size, corresponding to the artery bearing the same name.

\section{I'he Internal Iliac Vein}

Is extremely short-in fact it is nothing more than the common root of the two venous trunks by which it is said to be formed, viz. the ischiatic and lateral sacral veins. Its situation is the entrance of the pelvis, a little outwardly to the sacro-iliac symphysis.

THE ISCHIATIC VEIN, situated upon the side of the pelvic cavity, midway between the external iliac and lateral sacral veins, consists of a short but bulky trunk; and of a collection of large branches uniting to form it at the upper opening of the great sacro-sciatic notch. These branches may be distinguished into an internal and an external set. The internal comprise veins coming from the bladder, anus, perineum, and, in the male, from the bulb and prostrates-in the female, from the vulva and corpora cavernosa vaginæ. The external consist of veins coming principally from the gluteal and obturator muscles.

THE LATERAL SACRAL VEIN originally comes from the tail, being formed there by two or three slender coccygeal veins; it runs forward along the side of the sacrum, receiving other veins in its course, viz. 1. Perinenl veins, a collection of small branches coming from the back of the thigh, gluteal muscles, perineum, and anus. 2. Sacro-spinal branches, small veins issuing from the spinal canal, through the internal sacral foramina.

\section{The External Iliac Vein}

Is found running along the inner and upper side of 
the external iliac artery, taking, indeed, all the way precisely the same course as the artery. It extends from the bifuncation of the posterior vena cava to the place where it leaves, in company with the artery, the brim of the pelvis, the vein after that beconing femoral. As it departs from the cavity of the belly, this vessel receives the

IYGUINAL VEIN, one of rather large size coming from the groin, which owes its formation to a considerable branch energing from the muscles of the thigh; and a superficial or cutaneous abdominal vein, which runs in a serpentine manner along the abdomen, after taking its rise as far forward as the cartilages of the ribs, where its branches form communications with the cutaneous veins of the thorax : it is a very conspicuous vessel in milch cows, and is vulgarly pointed out as the milk vein.

\section{The Femoral Vein}

Is the continuation of the iliac trunk below the brim of the pelvis; and the main chamnel into which the deep-seated veins of the hind extremity pour their blood. I shall commence my description of the veins of the hind extremity from below, having already proceeded so with those of the fore extremity; but it will not be necessary for us to descend so low as the foot, for the plantar veins are similar both behind and before: we may begin therefore at the leg.

THE LARGE METATARSAL vein having ascended about half-way up the cannon, by the side of the flexor tendons, leaves them and takes an oblique course over the bone to reach the anterior and inner part of the hock, where it sends down an anastomotic branch un-

PART IY: 
der the tendon of the flexor metatarsi, from which anastomosis with the small metatarsal vein, and from the accession of a branch coming from the hock, results the

Anterior trbial vir or veins, for there are commonly two veins accompanying the anterior tibial artery. They run between the tibia and fibula to the inferior and posterior part of the os femoris, and there are joined by the posterior tibial vein, and all three unite to form the femoral.

The POSTERIOR TIBIAL VEIN is a continuation of the small metatarsal vein, which latter corresponds in comparative size and course to the small metacarpal vein. It runs in company with the posterior tibial artery, receiving various muscular branches in its course and also the medullary vein of the tibia.

THE FEMORAL VEIN is one of large size. It begins at the place of union of the two last-described vessels, runs behind the femoral artery, and ends in the external iliac vein. It receives various large muscular veins, corresponding to the principal branches of the arterial trunk, as well as veins from the stifle-joint, and the medullary vein of the os femoris. Also, about twothirds of its length upward, it is joined by the saphena vein.

The vena saphena Major, the principal superficial vein of the hind extremity, takes its rise, at the inner and fore part of the hock, from the large metatarsal vein, where a branch from this trunk ascends obliquely round the inner side of the joint to join the vena saphena minor. It then makes an oblique ascent up the middle part of the thigh, covered only by the skin, and higher up plunges inward among the muscles to join the femoral trunk. At the hock, it has a 
remarkable anastomosis with the anterior tibial vein, thus establishing a communication between the deepseated and superficial veins: it also receives many cutaneous and muscular branches in its course; but these are too variable in their number and distribution to come very usefully into any general description.

THE VENA SAPHENA MINOR springs from the small metatarsal vein. It runs directly up the back of the hock, over the root of the os calcis, and pursues its course upward along the front of the gastrocnemii, and ultimately joins the femoral vein. It anostomoses with the greater saphena, and receives in its course both cutaneous and muscular branches.

\section{The Vena Porta.}

TнE veins of the chylo-poietic organs have a peculiar and distinct arrangement from those of the body in general, and on this account a separate description is commonly given of them. They receive blood, as we have already seen, in a similar manner to other parts, but they return their blood into a channel which conveys it all through the liver, instead of conducting it immediately into the rena cava posterior; this channel is the vena portæ, a vessel principally formed by the union of the splenic and mesenteric veins, though it is contributed to also by veins coming both from the stomach and pancreas.

The splentc vein, in company with its artery, occupies the fissure of the spleen, makes a circuit in a flexuous manner towards the right side, receiving in its way branches from the stomach, omentum, and pancreas, besides all the veins of the spleen, and at length ends in the vena portæ. 
The MESENTERIC veINS, anterior and posterior, bear much corresipondence to their arteries. The anterior one is of large size, being constituted of those numerous ramifications which are distributed over the small intestines together with the arteries; it also receives branches from the coecum and coecum caput coli, some few from the stomach, and some from the pancreas. The posterior mesenteric runs obliquely forward, after having received branches correspondent to those of its fellow artery.

The vena porte, at its origin, is concealed by the pancreas, immediately above which its formation takes place. From this, it runs under the beginning of the duodenum, on the right of the hepatic duct and artery, to the concave part of the liver. About opposite to the centre of the right lobe, the vein splits into two divisions: the right immediately enters the substance. of that lobe'; the left is continued forward with the hepatic artery, and, as that vessel does, bifurcates to supply the left and middle lobes. 


\section{ISCIURE LXXIII.}

\section{Distribution of the Absorbents.}

I HAVE already spoken in general terms of the structure and physiology of these vessels, and given an account of their diseases; it only remains for me, therefore, to point out their distribution. Prior to commencing this inquiry however, I shall make a few observations on their ramification, and point out the methods usually adopted to discover them.

The absorbents in general from every part of the body become ultimately united into two main trunks, by which the absorbed fluids are poured into the large veins in the vicinity of the heart. Generally speaking, they accompany the veins; but they are comparatively minute to those vessels, vastly more numerous and valvular, still less constant in their calibre and particular distribution, and, lastly, form more frequent anastomoses and tortuosities in their course. Furthermore, they perforate or end in and take fresh origin from lymphatic glands in their way; though some terminate in the contiguous venous trunks: some, indeed, even retrogade for a certain distance; while others split into two or more branches which re-unite farther on and form once more a single tube. 
The lymphatic glands are lodged in various unexposed places in the body, enveloped in loose cellular and adipose tissue, which renders them moveable to a certain extent in accommodation to the motions of the parts to which they are attached. We find these glands, commonly in groups or clusters, sometimes solitary, under the tongue, in the throat, along the neck, in the axillæ, about the roots of the bronchire, within the mediastinum, at the roots of the mesenteric vessels, within the pelvis, and in the groins.

The superficial and deep-seated lymphatics communicate frequently with one another, and both send off anastomotic branches to whatever solitary absorbents there may be running in the vicinity; but the glands themselves are mutually linked together by many ramifications which run from one to another, and form thereby a continued series of communications.

The following are the best methods of demonstrating the absorbents*. In an animal opened alive, some hours after it has been fed, the lacteals are seen turgid with chyle; they may also be made visible by throwing colored thin fluids into the intestines; or, by making ligatures on the trunk of the anterior mesenteric artery, which must necessarily include the trunk of the absorbents. "An eye accustomed, readily distinguishes lacteals upon the intestines from arteries and veins, even when they are collapsed and empty: punctures may be made with a lancet, and the vessels injected with quicksilver by means of a tube formed expressly for that purpose. I have sometimes injected lacteals from punctures made by the sides of veins, where $I$

* These methods are drawn from the accurate researches of Mr. Cruiksuank. 
knew they must be, though they were then invisible to the naked eye."

Upon the liver and lungs lymphatics are frequently visible, and may be injected by poncturing one of the small branches; but the valves almost always make the injecting of them from the trunk to the branches impracticable. Pressure in the course of the absorbent circulation will commonly force from the extreme branches into the trunks some little reddish or brownish fluid, making the latter visible, which may then be punctured and injected.-Watery fluids thrown into the arteries, veins, or ducts of glands, very commonly get into the absorbents and render them visible.-One of the best methods is to previously inject the arteries and veins of the part, and afterwards macerate it for some days; putrefaction then takes place, air is generated in the cellular membrane, whence it gets into the orifices of the lymphatics, and uniformly fills their branches.-The best subject for these injections is one whose limbs are without fat and are dropsical, but not too much so.

In parts where glands are to be found, it is only necessary to puncture the gland and introduce a tube filled with quicksilver, or push the pipe into its substance without any previous puncture. The mercury thus fills the cells of the gland, and from these the lymphatics. "The thoracic duct itself is most successfully injected in the same way, that is, either from glands upon the mesentery, upon the bodies of the lumbar vertebræ, or those upon the inside of Poupart's ligament." "When vessels are injected, and very much resemble lymphatics, the best method of determining whether they are or are not lymphatics, is to trace them to the 
nearest lymphatic glands: if they terminate in them in the usual manner, they are lymphatics."

No English veterinarian, to my knowledge, has up to the present day been at the pains to demonstrate practically the particular distribution of the absorbing vessels of the horse; we have hitherto travelled onward analogically, and so, I have a notion, should we continue to do, had not our French professional contemporaries furmished us, as well as themselves, by their praise-worthy exertions in the cause of science, with materials to fill up this lamentable hiatus in veterinary anatomy: Professor Gira R D, whose "Traité d'Anatomie Vétérinare" does no less credit to the talent and industry of its author than honor to the veterinary school over which he presides, has presented us with an article on the ramification of the lymphatics which I shall translate, and take the liberty to transcribe into this lecture.

\section{"The Thoracic Duct.}

"Тне largest, longest, and most remarkable of the lymphatic vessels, in which terminate the majority of the lymphatics of the body, is situated within the thorax, on the right side of the dorsal vertebræ, between the aorta and the vena azygos; it receives the lymphatics from the posterior extremities, pelvis, parietes and viscera of the abdomen, head, neck, withers, and left anterior extremity.

"It takes its origin under the loins in a dilatation or sinus, very variable in its form and dimensions, situated at the root of the great mesenteric artery, and named the receplaculun chyli; it directs its course forward, enters the thoracic cavity by the aortic perforation through 
the diaphragm, extends along the bodies of the dorsal vertebræ until it arrives opposite to the base of the heart, where it curves downward to cross over to the left side, in its way to the anterior opening of the thorax, and, as it leaves the spine for this purpose, runs obliquely over the trachea and esophagus; having reached the left side, it stretches forward to the beginning of the anterior vena cava, and terminates, against the middle of the anterior border of the left first rib, in the base of the left axillary vein: not infrequently, it ends in the right axillary; in some instances, even, in the beginning of the anterior cava. At its termination it dilates and forms a sinus, whose mouth, opening into the vein, is guarded by a broad valve so disposed as to prevent any, reflux of blood into the duct *; it has also a ligamentous band around it at this part which confines it to the vein receiving its contents.

" This canal shows but little uniformity in its calibre; in some places it is strait, in others varicose: it is not uncommon to find it detaching one and even several branches in its course, large or small, which either remain separate, or else after running a certain distance rejoin the main channel.

\section{"The Receptaculum Chyli.}

“This reservoir, (also named 'la citerne lombaire,') forms the point of general confluence of all the lymphatics of the posterior limbs and abdomen, and that from which originates the thoracic duct: it is maintained by the aorta on one side, the vena cava posterior on the

* "Nutwithstanding this valve, blood often gains admission into the canal; this is ulservable in all cases of violent death, or in which urugges and convulsions attend expiration." 
other, and is formed by the union of five or six large lymphatics, of which two or three come from the entrance of the pelvis, two or three others from the mesentery, a single one from the environs of the stomach and liver."

The Professor here makes a classification of all the lymphatics of the body into those that proceed to the abdominal and those that run to the thoracic portion of the duct.

“I. Lympiatics discharging their Contents into the Abdomina Portion of the ThoRACIC DUCt.

"1. Lymphatics of the Posterior Extremities.

"THESE are distinguishable into the superficial and deep-seated. The first originate particularly from the skin and subcutaneous cellular tissue. They form divers ramifications which accompany the superficial veins; of which the most remarkable attend the vena saphena major, frequently anastomosing with one another, and forming altogether an anastomotic network. All these lymphatics run to the subcutaneous inguinal glands, which are lodged upon the superior and anterior part of the thigh.

"The deep-seated lymphatics take their rise from the foot, ascend along with the plantar veins, continue upward among the muscles in company with the deepseated veins, corresponding in their principal divisions to those vessels, and proceed to the inguinal glands.

"All the lymphatics of the posterior limbs assemble at these glands, and here form a plexus, from which several large branches depart and traverse the iliac clands, clinging to the sides of the iliac vessels, and 
discharge their contents into the pelvic branch contributing to the receptaculum ehyli.

\section{"2. Lymphatics of the Pelvis.}

" $\mathrm{T}_{\mathrm{HE}}$ vessels coming from this cavity run in part to the inguinal glands and in part to the internal pelvic glands. The superficial lymphatics about the pubes and the outlet of the pelvis rum and join those of the extremity; those of the perineum and anus enter the cavity, and are accompanied by those coming from the croup and tail, both proceeding to the glands within the interior of the pelvis. All the deep-seated lymphatics accompany the veins, make for the pelvic glands, form unions with the others, and run and empty themselves into the main pelvic branch, wherein their lymph mixes with that coming from the inguinal glands.

"The lymphatics of the urinary and genital organs, included in the pelvic cavity, also traverse the glands lodged therein, and unite with those of the parietes of the pelvis. Those of the scrotum enter the inguinal glands, as also do those belonging to the sheath and penis. The ramifications derived from the testicle and spermatic cord take the course of the veins, and penetrate one or two of the lumbar glands lodged at the entrance of the pelvis. The lymphatics of the mammæ, which are also divisible into superficial and deep-seated, run to the inguinal glands, and anastomose with the superficial set belonging to the inferior parietes of the abdomen; but, before they reach these last glands they pervade those of the mammæ.

"3. Lymphatics of the Parietes of the Abdomen.

"THESE vessels, in general but little developed, for 
the most part run to the inguinal glands. The superficial set of the lower parietes accompany the cutaneous (inguinal) vein, anastomose with the lymphatics of the scrotum and manima, and traverse the glands in the groin : some of them direct their course forward along with the cutaneous (external thoracic) vein of the thorax, unite with the superficial lymphatics of that part, and proceed to the axillary glands. The deep-seated vessels of the belly run in company with the epigastric vein, and go to the inguinal glands, or else they accompany the pectoral vein, and pervade the glands in front of the thorax.

"The superficial or subcutaneous lymphatics of the loins join either those of the croup or those of the flanks : the deep-seated, which spring from the peritoneum, muscles, or spinal canal, perforate one of the internal lumbar glands, and pass onward to terminate in the main pelvic branch.

"Among the lymphatics of the abdominal surface of the diaphragm, those issuing from the peritoneum and muscular texture make almost all for the main hepatic branch; some others take the course of the phrenic veins and form a union with those of the thoracic side of the muscle.

\section{"4. Absorbents of the Mesentery.}

"THe mesenteric branches, ordinarily two or three in number, the most considerable of which is constantly united to the great mesenteric artery, receive all the vessels continued from the mesenteric glands, as well as those coming from the mesentery and intestines.

"The mesenteric absorbents, extremely numerous, are sustained between the layers of the mesentery 
where they form a vascular network ; many of them issue from the exhalent surface of the mesentery and intestinal tube; others take their rise from the interior of the intestine, from which they imbibe chyle. All these vessels converge towards the lymphatic reservoir, clinging in their passage around the mesenteric veins; some however taking a solitary course at a greater or less distance from any bloodvessel: having arrived at the root of the mesentery, they pass through one or two, sometimes three, of the mesenteric glands, and afterwards join the principal lumbar lymphatics. The absorbents of the cœcum and cœcum caput coli run to the glands set at intervals along the intestinal tube, whence they procoed to the receptaculum chyli.

"5. Lymphatics of the Liver, Stomach, Spleen, and Omentum.

"The hepatic trunk comprises the lymphatics issuing from the liver, stomach, spleen, and omentum : this branch of the receptaculum chyli not uncommonly consists of two divisions, and receives, in addition to the above-mentioned vessels, many ramifications from the crura of the diaphragm.

"T'he lymphatics of the liver, extremely numerous, are distinguishable into a superficial and a deep-seated set. The first arise more particularly from the exhalent surface of the organ, creep along upon its peritoneal capsule, and there form a plexus of close and intricate network. Those from the anterior surface make one or two large branches which perforate the diaphragm, enter the thoracic cavity, unite with the lymphatics from the cordiform tendon, and proceed to the fore part of 
the thoracic duct; whilst those of the posterior surface enter the glands placed around the great fissure, where they unite with the deep-seated set.

"The deep-seated hepatic spring from the parenchyma of the gland, cling around the divisions of the hepatic artery and hepatic veins, issue from the interior of the viscus by the great fissure, run to the glands there, and afterwards proceed with the superficial to the main hepatic trunk.

"The lymphatics of the stomach, of which the superficial come from the external surface, the deep-seated from its cavity, follow the veins, and are distinguishable into a superior and an inferior set. The former take the direction of the lesser curvature, perforate the glands thereabouts, and go to join the absorbents of the liver; the latter run over the greater curvature, enter the glands arranged along the fissure of the spleen, form communications with the lymphatics of that viscus and those of the omentum, and depart to contribute to the hepatic trunk.

"As to the lymphatics of the spleen, the superficial, like those of the liver, come from the periphery of the organ and enter into a thick and complicated network; the deep-seated issue from the interior, and contract with the former numerous anastomoses. Along the fissure of the spleen these vessels become united, form large branches which follow the course of the splenic veins, and anastomose with the lymphatics of the great curvature of the stomach; both sets then run to the great fissure of the liver, and there terminate in the hepatic trunk.

"The lymphatics of the omentum accompany the ve- 
nous ramifications, and either join those of the great curvature of the stomach, or the superficial absorbents coming from the cœcum caput coli; those around the pylorus anastomose with the pancreatic, and accompany them to the hepatic trunk.

"The lymphatics of the pancreas also run with the divisions of its veins, and join either those of the liver or those of the spleen; some proceed directly to the common hepatic trunk.

"Besides these three portions or lymphatic trunks which constitute the receptaculum chyli, the abdominal portion of the thoracic duct also receives the lymphatics of the kidneys and renal capsules. These vessels, distinguished into superficial and deep-seated, perforate the glands placed, internally, next to the parts from which they spring, and empty themselves into the superior side of the thoracic duct.

"II. Ramifications terminating in the Thoracic Portion of the Maln Common Duct.

"THss, the terminating portion of the thoracic duct, receives the lymphatics transmitted from the internal dorsal, bronchial, and cardiac glands, and those either from the left axillary glands, or from the sublingual and guttural glands. In this numerous series we find the lymphatics of the parietes and viscera of the thorax, of the head, neck, and left and anterior extremity:

"1. Lymphatics of the Parietes of the Thorax.

"THE superficial absorbents of the chest take their rise either from the surface of the skin or else from the subcutaneous muscles; they form several large 
branches which accompany the thoracic cutaneous vein, unite with the superficial lymphatics coming from the anterior parietes of the abdomen, and proceed to the axillary glands.

"The deep-seated set take divers directions and pass through different sets of glands. The pectoral, which anastomose with ramifications from the abdomen, follow the pectoral vein, and reach one or two glands at the entrance of the chest. The intercosial spring from the pleura and intercostal muscles, accompany the intercostal veins, pervade the internal dorsal glands, and terminate by several branches in the thoracic duct. The lymphatics of the fleshy part of the diaphragm unite, some with the posterior intercostal, others with the pectoral; those coming from the crura run to the dorsal glands, where they anastomose with the intercostal; those from the cordiform tendon anastomose with the deep hepatic, run forward between the layers of mediastinum, nearly to the heart, and enter the cardiac glands.

\section{"2. Lymphatics of the Thoracic Viscera.}

"THE absorbents of the different organs contaiued within the thorax, traverse one or several of the bronchial or cardiac glands, and afterwards form divers: branches which end in the thoracic duct. The pulmonary lymphatics, very numerous, are distinguished into superficial and deep-seated. The first take their rise from the surface of the lungs, creep along under their enveloping membrane, and make for one or more of the bronchial glands. The deep set, which originate from the air-cells and from the arcolæ of the parenchymatous tissue, follow the divisions of the pulmonary veins, run to the root of the bronchiæ, there unite with the 
superficial, and perforate one or two of the bronchial glands.

". The cardiac lymphatics derive their origin, either from the surfaces (both exterior and interior) of the heart, or from the muscular substance of the organ; they mount upon the curvature of the posterior aorta, and disappear in the cardiac glands.

"The lymphatics of the superior part of the mediastinum and of the esophagus join, some the intercostal, the others the bronchial; those coming from the anterior part of this membranous partition, from the thymus, trachea, and esophagus, unite, either with the pectoral, or else with the cardiac and anterior intercostal.

\section{"3. Lymphatics of the Head.}

THE lymphatics of the head form two planes, a superficial and a deep one. The superficial pursue the course of the cutaneous veins, and run in part into the sublingual, in part into the guttural glands. The deep vessels, which come from the nostrils, fauces, palate, stc. also run to the sublingual and guttural glands, in which they unite with the superficial. From these two groups of glands, through which pass the lymphatics of the head, depart several large branches, two or three of which descend upon the anterior face of the trachea; others follow the passage of the deep and subcutaneous veins, unite with those of the neck, and descend to the front of the chest. Almost all these vessels terminate in the thoracic duct; some few atone on the right side ending in the right axillary trunk.

:PAR'II. 
"4. Lymphatics of the Left Fore Extremity.

"THE lymphatics of this member present the same disposition as those of the posterior limbs, and are divided into superficial and deep-seated. The former, consisting of divers ramifications, accompany the superficial veins; the more considerable of them forming a plexus which accompanies the cutaneous (superficial brachial) vein of the limb. The deep vessels originate from the foot, muscles, and bones, pursue the divisions of the deep veins, and plunge into the axillary glands, wherein they unite with the superficial, and whence they extend to the thoracic duct.

"The Right Terminating Trunk of the Lymphatics.

"THis very short lymphatic canal is obliquely situated at the entrance of the thorax, upon the transverse process of the last vertebra of the neck, extending in a direction from above downward and from without inward, and terminating most commonly in the right axillary vein; though in some instances it joins the thoracic duct, or else ends close by the side of it.This lesser trunk is formed by the lymphatics coming from the right axillary glands, and some from the right lung, and right side of the neck and trachea." 


\section{LECTURE LXXIV.}

\section{On Fever.}

\section{LEST the title of this lecture may startle the dis-}

ciples of our present school of veterinary medicine I beg to qualify it in limine by premising, that it is my intention herein fully and deliberately to investigate the subject with a view of coming to safe practical conclusions on two long-standing and still-pending questions of very great interest and importance to us, viz. Are horses liable to fever? And, if they are, what is the nature or kind of fever to which they are subject?

These are two very plain questions, and one would think, after between 30 or 40 years' experience, questions that might be promptly, plainly, and satisfactorily answered; so far however are the accounts, both written and oral, from being conclusive that I know of no subject about which there is more wavering, nay, discordance and discrepancy of opinion, than that of fever. Ancient veterinary works yield nothing of interest on the subject; most of the old English writers on farriery, as well as the French, give descriptions of fevers of various kinds; Messrs. Feron, White, and BLAINE treat of fevers; Professor PEAlL, though his 
work includes the principal diseases to which horses are liable, devotes no part of it to fever; Professor CoLEMAN, when I was a pupil, appropriated no lecture in the whole course to the subject of fever. In order that we may set about this investigation with minds in some degree prepared to meet the subject, comprehend its various bearings and relations, and ultimately draw up judicious and safe answers to the foregoing questions, let us first take a cursory view of what the most eminent human nosologists have defined fever to be.

Our word fever appears to have come to us from the French word fierre, for the two are very like, and the latter is derived from the latin noun febris, which is related to the verb ferveo, signifying $I$ am hot: an origin that is perfectly accordant with the symptom most likely to attract the attention, namely, the increased warmth perceptible in the skin. Here we perceive an analogy between the words fever and inflammation; the latter coming from the Latin noun flamma, flame, and that again from the Greek verb $\phi \lambda$ ś $\gamma \omega$, which signifies $I$ burn, as if the one was a local heat or burning, the other a general heat; and this, in point of fact, we shall find to be the case. We must not run away with the idea however, that the essence of fever consists in increased temperature of body; for this is an indication that has frequently been known to be absent during the whole course of the disease, and when present is only to be regarded as one of its prominent or characteristic symptoms. But, let us see what the most eminent physicians have set down as the signs or symptoms collectively constituting fever; for it has been correctly observed by modern pathologists, that, so far from any one of them being essential 
10 its existence, each has been known to be absent, and all occasionally present, where there has been no fever.

BOERHAAVE, the late distinguished professor of medicine at Leyden, says, in his Aphorisms - "In every fever, arising from internal causes, there is always $a$ shivering, a quick pulse, and heat, varying in degree at different times of the fever." And further on-"These symptoms indeed are present in every fever, but the Q 1 ICK P $\mathrm{Pr}$ ISE alone is present through its whole course, from the beginning to the end, and by that only the physician judges of the existence of fever."

Dr. CLLLE, the late learned professor of medicine at Edinburgh, in his Systema Nosologicum, has arranged five orders under his class pyrexice, or febrilc diseases, viz. I. Febres, simple idiopathic fevers. II. Phlegmasice, inflammations attended with fever. III. Exanthemata, eruptive fevers. IV. Homorrhagice, hemorrhages attended with fever. V. Profluria, fluxes attended with fever. His general characterization of pyrexiz is this-" Post horrorem, pulsus frequens, calor major, plures functiones læsæ, viribus presertin artuum imminutis*." To his first order, comprising fevers properly so called, he gives this definition"Prægressis languore, lassitudine, et aliis debilitatis signis, pyrexia, sine morbo locali primario.$+ "$

Dr. FORDYCE, in his "General Doctrine of Fevers," presents us with a description of an ague, a febris intermittens of Dr. CULLEN, as a specimen of pure fever,

- "After shivering (or a sense of coldness) a quick pulse, increased heat, disturbance of several functions, prostration of strength."

$\uparrow$ "Pyrexia, without any primary local disease, following lan. enor, lassitude, and other signs of debility." 
of which he considers the other's (as indeed did Dr.

CULLEN before him) as variations. Now, an ague is a periodical fever characterized by three stages, viz. a cold, hot, and sweating stage or crisis: the symptoms I subjoin in a note in order that we may have this assumed prototype of human fever before us*.

By these eminent physicians continued fevers were regarded as variations of the intermittent; indeed, as I

* Fever generally commences with "languor, weariness, weakness, diminished sensibility, cold and trembling, pain in the back. Rigor, horror, horripilatio, (the technical terms for shivering) succeeds; the skin becomes pale and dry; the tongue, dry and foul, with thirst; transparent urine; costiveness; and suppression of other secretions; pain,in the limbs, joints, and forehead-delirium. Anxiety follows; oppression and swelling about the præcordia; frequency of pulse; quick and laborious respiration; thirst, flatulencies, loss of appetite, nausea and vomiting. Unless these, the symptoms of the first stage, destroy the patient, they are followed by the second stage or hot ft, consisting in-Rigor and horror, heat arising from the præcordia and diffused from thence over the body irregularly, unequally, and with flushing; a strong, full, obstructed pulse; or a very frequent, small one; pain in the head, and joints; stupor and dilirium; universal soreness; redness arising in different parts irregularly; the urine high-colored, but perfectly transparent; sweating in the head and breast, or over the whole body; partial secretions. - The symptoms of the first stage are gradually relieved. At last-the crisis-the pulse becomes free; all the secretory organs are relaxed; hence the skin grows soft, and moist, and returns to its natural color; the tongue likewise is soft and moist; the belly is open; and the urine is discharged in greater quantity; if transparent when voided, after a little time it becomes turbid and opaque, and at last depusits a cupious sediment: the secretions are often greatly increased; there arises a copious and universal sweat, or a purging, or a great Alow of urine."-Elements of the Practice of Medicinc, hy Geo. Fordyce, M.D. F.R.S 
observed before, all febrile diseases were referred to the paroxysm of this one as the archetype. And this appears to have been assumed as the standard by which we are to determine the question of the occurrence of fever in horses. But, what can be more unpathological than such a notion as this? In the human frame even, fever puts on such various aspects that (as an ingenious writer remarks) "fancy rather than sound observation" seems to have led to the above ascription of such affinity between them; but, to pretend to make a human intermittent the prototype of fever in horses, is surely stretching analogy in medicine to an unwarrantable extent! If we can prove that fever in horses is essentially the same in its nature, so far as we can judge from its principal symptoms, its general causes, its common progress and termination, we do all in my mind that is required to establish its existence; but, we can do more, its symptoms individually will even admit of a comparison with those of human fever, and to me too plainly indicate the same derangement of the system at large to be reasonably ascribed to any other origin.

There is a fever to which man is more subject than to any other, commonly called inflammatory fever, technically named by Dr. Culden, synocha, and thus defined by him-"Calor plurimum auctus; pulsus frequens, validus, et durus; urina rubra; sensorii functiones parum turbatæ*." In fact, this is a fever characterized by a greater development of the hot stage of the intermittent; for the cold stage is here frequently absent altogether, and when present is always considerably less marked; and the hot fit is not followed by any

* "Heat, much increased; pulse, frequent, strong, and hard; nrine, high-colored; sensorial functions, but little disturhed." 
regular sweating stage. During its progress, remissions may be evident or not; and the inflammatory excitement may continue all through, or the latter stages may be marked by symptoms of weakness and nervousness, which belong to a fever of debility denominated typhus.

The numerous forms of human fever distinguish themselves in practice into two general classes: those arising from causes operating on the system at large, or that originate without any apparent cause, denominated primary or idiopathic fevers; and those arising from inflammation or any other local affection of a particular organ, hence named secondury or symptomatic fevers.

With these observations before us on the definition, symptoms, and classification of human fever in general, I shall, prior to submitting opinions practice has long established in my mind, make a few extracts from the most popular veterinary authorities on the subject of fever in horses.

Gibson makes three classes of fevers: simple continued fevers, compound fevers, and epidemic fevers. The first, he says, "appears from the writings of Seignior Russixi, SOCLEYSEL, and other foreign authors, to be very frequent in hotter climates; and I have frequently met with it among our own horses, in the summer especially, when the weather has been hot and sultry, and the air much rarified." He ascribes their origin to hot and stimulating liet, and certain atmospherical temperaments, "for I have known," he adds, "several horses in this fever, at the same time, and in different places, where no other visible cause could be assigned for it." The symptoms he gives, are-restlessness, beating at the flanks, eyes red and inflamed, tongue parched and dry. The horse looks lively instead of being dull, and is "very attentive to any noise," the reverse of which is the case in complicated fevers. Loss of appetite. "His ears and feet generally feel cold, or alternately hot and cold."-Other parts commonly hotter than ordinary. Urine 
high-colored. Thirst.- "Complicated or compound fevers are denuminated malignant, putrid, or pestilential."-"Malignant fevers are not unconmon in horses, though few practitioners, for waut of a competent knowledge in such things, have been able to distinguish them from severe colds, or other diseases attended with feverish symptoms."

Bracken, Gibsun's contemporary, thungh he does not question -indeed lie acknowledges the occurrence of the other fevers inentioned by GIBson, only however gives a detailed account of one which he calls symptomatic fever, though it is evident, that, according to the views entertained by the furmer writer, it is also idiopathic; for he says that very hot weather, suppressed perspiration, \&c. may bring it on. The signs of fever, according to this author; are " violent heat, and fulness of the vessels, a beating of the lieart and flanks much quicker than ordinary; a dryness in the mouth with roughness of the tongue; continual watchfulness and restlessness." He concludes with saying,- "I think it needless, to enumerate all the different kinds of fevers, such as putrid, pestilential, hectic, intermitting, and the like."

Bartlet, whose compendious and pithy work contains no more concerning fever than the treatment, observes that "warm clothıng, with dilution, and abstinence from diet, will go great lengths; but when bleeding and gentle evacuants are joined to these, the end is generally accomplished in a few days."

That keen and clever but censurious writer, $\boldsymbol{T}_{\text {A PLIN }}$, commences his description of fever thus truly and remarkably. "The observations and reflections that first induced me to perceive the absolute necessity of some reformation in the practice of farriery, never iufluenced me more in a conviction of that want than did a thorough invostigation of the subject before us." He divides "febrile disquietudes simply into two kinds, the symptomatic and the inflammatory."-" The pathognomonic or certain signs are, a universal heat and disordered pulse, a palpable disquietude and measiness; shifting from place to place; the horse labors under difficult respiration, his mouth is very dry, his tongue is parched and hot, he declines food, but receives water: sometimes seizes his hay with seeming eagerness, then drops it in disappointment; the body is generally costive, and in the early state of the disease there is a proportional obstruction of urine." 
Ferow says, "Horses are subject to one species of fever only.But when a horse is taken with a fever, it is much more dangerous than when that mulady risits man.-Fever in animals is seldom a primary disease, being always associated with and originating from a variety of diseases, such as catarrh, strangles, pneumonia, fractures, \&c."

Wнгте says, "The fevers of horses bear very little analogy to those of the human body, and require a different treatment.-I can distinguish only two kinds of fever, the one idiopathic or simple; the other, symptomatic. The simple fever does not occur so frequently as the symptomatic, nor is it by any means so formidable in its appearance.-The following are the symptoms of simple fevershivering, succeeded by loss of appetite, dejected appearance, quick pulse, hot mouth, and some degree of debility : the horse is generally costive and voids his urine with difficulty. The disease is often accompanied with quickness of breathing, and in a few cases, with pain in the bowels, or symptoms of colic.-When a pain is symptomatic it is not perceived by shivering, nor is it so sudden in its attack as simple fever. When the symptoms meritioned are joined by difficulty of breathing and quick working of the flanks, with coldness of the legs and ears, we may conclude that an inflammation of the lungs is the cause of fever."

Mr. Blarne brings up this cursory exposition of the opinions of our latest veterinary writers who have treated on fever, and observes, "that fever in the horse does very seldom exist as a primary affection; and that it still less frequently remains in an original idiopathic state without being transferred to some one particular organ. Mr. B. divides fevers into simple or idiopathic, epidemic or catarrhal, malignant epidemic or catarhal, and symptomatic." In re. gard to the first, Mr. B. observes-" Pure fever is certainly a rare occurrence in the horse; but I am disposed to think that it is not: so much its extreme rarity which renders its existence disputed, as that his constiutional tendencies are such as seldom allow general fever to prevail in him, without a more active and local translation of the inflammation to some vital organ.-In my own practice I have ccrtainly met with this (inflammatory) fever.-The symptoms that present themselves are, first, a shivering fit, in which the skin is corrugated, and shakes violently; the hair stares, and the lega, ears, and muzzle, feel intenscly cold-Tu thesc some 
degree of heat succeeds, and the horse resumes nearly his usual habits; until the second cold fit occurs, after which the pulse becomes increased in frequency and fulness. The skin is found alternately hot and cold, with occasional partial sweatings; the bowels rumble and are flatulent, and general uneasiness without acute pain is manifested by restlessness and frequent shitting of position. The inner surface of the eyelids and nostrils is slightly increased in redness; thirst is present, and costiveness is also common, but the respiration is not greatly accelerated." Should this, the first stage of fever, not prove to be the prelude to any local disease, but remain "purely idiopathic, which, though very seldom, does now and then happen, a series of symptoms supervene, seldom invariably the same in any two subjects, but with sufficient general characters to describe them as follows:-The pulse continues to have a corded feel, but loses still more of its fulness and increases in quickness; the skin also becomes moist, the eyes weep and appear red and inflamed; and while the nose secretes a thin acrid fluid, the mouth is usually hot and dry. The urine at first is gradually secreted in larger quantities, and becomes of an opaque color. It is not unusual also for the hind legs to swell, and sometimes likewise serous deposits take place about the head, the throat, or along the chest and belly; and when these occur early in the complaint, I should consider them in this, as in the epidemic catarrh, rather as favorable indications; that is, I have found the irritative fevers of the horse, so accompanied, more tractable than others without: but I think I have observed the reverse of this to be the case in the fevers in horned cattle.-What I have just described may be considered as constituting the middle and principal stage of this fever, to which, under favorable circumstances, there succeeds a softer and less frequent pulse; the countenance looks more lively; and although the muscular weakness rather increases, the irritability lessens; the secretions also return to their natural state; the mouth feels rool and moist, and the heat of the body becomes lessened, as well as regular and equable throughout ; slight symptoms of returniug appetite likewise appear: under which circumstances a resolution of the fever is formed. An unfavorable termination I have never seen, and consequently I cannot describe the more mortal symp. toms."

Tu there accounts, I have considerable satafaction in having it in 
my power to subjoin the results of a similar investigation, contained in the French Veterinary Journal, in an ingenious article, entitled, "Existe-t-il, en médecine vétérinaire, des exemples bien constatés de fièvres essenticlles?" by the lamented son of the present Professor Granan, whose talents and professional ardor manifested great promise in the cultivation and improvement of veterinary science. He winds up a cursory review of the principal French velerinary writers on fevers with these conclusions.

“1st. That Solleysel, Garsault, Bourgelat (and DefabIERE Blaine), though they admit the existence of essential (idiopathic) fevers in domestic animals, leave us to discern, by the manner in which they express themselves, that they do not write from actual observation, but only conform to the general opinion.

"2d. That Lafosse and Volpi (veterinary professor at Milan) do not believe in its existence.

3d. That Vitet describes fevers as they are found in works on humah medicine, and that he has been followed by Rozier and others.

" 4 th. That the later authors who have mentioned fevers, are all copists, their readings being merely varied conformably to certain new doctrines of the day.

" 5 th. That the observations reported in the "Instructions Vet'trinaixes,' so far from offering any proofs of the existence of fever, militate in favor of a contrary opinion.

" 6 th. That the malignant, petechial, and typhoid fevers, and the epizootics of the eighteenth century, \&c. have been found, on examination after death, to be attended with marks of violent in. fammation in different organs, more especially in the digestive viscera.

" 7 th. Lastly, that we are not warranted, by the observations in our possession, in considering that fever can exist in domestic animals independently of organic lesion somewhere; that the word fever, whether it be used to denote diseases attended with danger, or a salutary effort of nature to free the system of some noxious agent, ought to be interpreted, a group of symptoms which indicate or represent more or less faithfully an inflammation. (local?) Recueil de Médecine Vétér. Cahier de Septembre, 1824."

M. Damotseau has presented us with a case of intermittent fever in the hurse, the only accredited observation of the kind. I 
believe, recurded in veterinary medicine, and one of considerable importance to us here, on which accounts I shall transcribe it.

" On the ofl of December, 1807, about three o'cluck in the afternoon, a stallion suddenly manifested inquietude. The pulse was small, contracted, and intermittent; mouth hot; tongue coated with a llackish fur; mucous surfaces saffiron-colored; painful tension of the right hypochondrium; stiffness about the spine; respiration short and laborious. This was followed by universal coldness; he stood all of a heap; the coat lost its gloss and stared; the pulse became almost imperceptible, and the eyes greatly downcast. After three quarters of an hour, the vital energies appeared revived; the coat shone, the pulse grew very quick. (90 in a minute.) This stage lasted nearly half an hour, and then he broke out into a profuse sweat; the pulse was full and soft, natural in frequency; the symptoms gradually disappeared; appetite returned, and vigor and animation. On the 6 th inst. at the same hour, another paroxysm. On the 7 th and 8 th, intermission. On the 9th, paroxysin less violent than the two preceding. From which day the paroxysms became irregular; the ague appeared to assume the tertian type; at length, it became quotidian : it left extreme weakness behind, and the recovery was not completed until March. Correspond. de Fromage de Fugré. Tom. iv. puge 48."

On this, and two other cases of minor interest, (one of meningogustric fever, the other of mucous fever,) M. GraArd comments thus. "The observations of MI. Damorseau are incomplete, inconvincing, and valueless, (sans force et sans valeur, ) because the subjects could not be anatomically examined, and because they appear to have been no more than cases, either simple or compound, of gastro-enteritis.-And we can much better explain the intermissions of fever by regarding them as the results of divers phlegmasire, than by considering them as idiopathic, since we have an inflammatory affection (specific opthalmia,) in which the intermission is well defined, and which nevertheless is never preceded and rarely accompanied by febrile commotion." Loc. cit.

Here, then, we have presented to us the observations and opinions of the principal veterinary writers, French and Italian as well as English, on the subject 
of fever: and it constitutes a mass of evidence altogether that I cannot, in justice to its authors, and in consideration of what $I$ have of $m y$ own to adrance, pass orer without comment. It is with considerable hesitation that I join in sentiment with GIRARn, and with him have to pass such a sweeping censure as he has done in his second corollary, that most of these writers, "s"expriment de manière à laisser entrevoir qu'ils n'en ont jamais observé eux mêmes, et qu'ils ne font qu'obéir à l'opinion générale;" but, really, when one comes to critically examine their accounts, and compare them one with another, one can hardly refrain from coming to the conclusion, that their descriptions were originally derived from human medicine, and have been but variously modified to suit the prevailing doctrines of the day, from the time of GiBson and BRAChEN and BARTLET. It is pretty evident that GrBsoN, to use the words of TAPLIN, "has gone through a system of imaginary fevers; and regularly transferred the observation and language of ancient authors upon the diseases of the human species to the constitution of quadrupeds." TAPLIN dismissed the complicated or compound-the imaginary fevers of these authors, and simply considered them as idiopathic and symptomatic; he however has passed by the main question without any practical remark whatever, and seems totally to have neglected the study of morbid anatomy. FERON quite staggers credit when he states that fever is much more dangerous in a horse than in a man. WHite has prudently abstained from "dabbling in troubled waters:" he has not forgotten however to bait his chapter on fevers with some antimonial powders, which he knew are sn grateful to the palates of his Newmarket readers, for 
which they ought, in veneration to his memory, out of common gratitude, erect him a statue in the middle of the round mile. I am sorry to see so respectable and experienced and persevering a practitioner as Mr. BLAINE, led away by the accounts to be found in various old authors of epidemic and malignant fevers, because the descriptions, unsupported as they are by the evidence of actual observation and morbid anatomy, are not of that stamp that deserve implicit reliance, and because modern practice, so far from confirming, goes directly to gainsay them. There seems no reason to doubt that epidemic, or rather epizootic and sporadic diseases have made such ravages as GIBSON and OsMer and several old French authors have reported, but there is great reason to doubt that these diseases were not idiopathic fevers: the symptoms, such as are put down to them, are mostly referable to certain phlegmasiæ, and their true seat and origin would have been discovered in all probability had these authors been more diligent inquirers after such practical facts as can alone render such accounts of value to us.

M. GIRARD, who has taken up the subject in a very proper and praise-worthy manner, and has manifested considerable penetration in reviewing the accounts of the French author's, is, it is plain to perceive, a disciple of M. Brocssars, who would place the fors el origo of fever in the stomach and bowels, and explain the phenomena of intermissions and remissions by asserting the intermittent character of the local inflammation, which Gira R further exemplifies by a reference to the specific opthalmia. But, we have no more reason, indeed, in my humble opinion, not near so much to give fever a habitation in the abdomen as we have to enthrone it 
in the head, with Dr. CLUTTERBUCK; for the brain, rather than the stomach, seems to stand foremost in the order of organic derangement. But, it would appear from the full range of observation, that no part of the body can be said to be insusceptible of inflammation in human fever, though, at the same time, no organ is invariably or exclusively affected: nay! indeed, some tell us that inflammation is not essential to fever at all -that it is merely a casual occurrence in fever! Be this however as it may, all I wish to contend for is, that both idiopathic and symptomatic fevers exhibit the same form, characters, and species, and require the same general means of cure ; and that, were it not for the local affection, it would be difficult or impossible to distinguish them. And if there be no apparent or discoverable difference in these fevers in a man, though we know that one proceeds from some local malady, while the source of the other is wholly a mystery to us, are we not, to reason at least analogically, warranted in ascribing the presence of an idiopathic fever to a horse, when the animal shows the same set of symptoms, without manifesting signs of local inflammation, which he exhibits after a violent local injury? However, it is not my intention to rest the question upon any negative evidence; and therefore let us now come fairly to the point, and see how far we can demonstrate it by the tests of actual practice.

We will suppose, for the sake of illustration, that a horse leaves his stable perfectly sound and well, but in the course of his journey "picks up a nail;" and that this nail penetrates the foot deeply, and occasions poignant pain with excessive lameness: I could scarcely have selected a more common case than this. Surl 
an accident, as every one knows, is speedily followed by acceleration of the pulse and respiration, by heat of skin, loss of appetite, derangement of the secretions and excretions, and sings of more or less sensorial disturbance. I should like to know by what name I am to designate this constitutional affection if I am not to call it fever? And, if I may be allowed to give this the appellation of fever, how an 1 to distinguish in my nomenclature the very sume set of symptoms when they arise without any appurent injury, or rationally assignable loral phlogosis? I say rationally assignable local disease because I know that this last by some will be called inflammation of the lungs, and for this sage reason, because the breathing is increased; but, was not the breathing increased in the case of the nail in the foot? And is it not likewise increased in gripes; and enteritis, and stagcrers, and all other acute and painful affections? There is as much reason to regard it as an inflammation of the heart as one of the lungs, and greatly more to consider it as an affection of the stomach or head, or of both. To show that symptomatic and idiopathic fevers are, at least in horses, one and the same disorder, they are continually running one into the other. The case of the nail in the foot is one in which the febrile originate at the same time with or very soon after the local symptoms; such also are burns, injuries of the head, \&ic. And, as Professor Thomson tells us, as local inflammation gives rise to constitutional febrile symptoms, so idiopathic fevers of all kinds, in their turn, often give rise 10 , or at least are accompanied by, local inflammatory symptoms. It is very possible therefore that a lever may prove the prelude to catarrh of pncumonia, indeed it is a common result

PAR'T 11 . 
since we know from practice that these affections are often so ushered in; but, for all this, fever is no more catarrh or pneumonia than it is gripes or staggers, as post mortem examinations will plainly show.

The fever, then, to which the horse is both idiopathically and symptomatically subject, is neither more nor less, than the synocha of Dr. CULLEN, common or simple or inflammatory fever. And so plain a point of practice is this, in my humble opinion, that we might as well attempt to deny the existence of inflammation in horses, as fever. What seems to have led to the denial has been, the over-eagerness to show the palpable absurdity of the accounts of the old writers: in very properly casting away such trash, we have overlooked the real state of the case; or, at least, we have not been patient and unbiassed practical observers, true and faithful reporters of our observations, but have suffered our minds to be prejudiced, and our opinions to be taken into bondage, by those to whom we are too apt to look up with oracular reverence. To contend, as GrRARD has done, that fever is meningo-gastritis, is to reason rather from consecutive disordered function than from general views of the case; and to prove that organic lesions have existed in the very few fatal cases of fever which are known to occur in any individual's practice, is not to demonstrate that such constituted the febrile disorder; or, why are such not found likewise in fever from a nail in the foot? The symptoms of these fevers are precisely similar; they are only characterized by certain superadded marks or expressions of local injury or disease, which define the principal malady to be of some other class, and the febrile connection to be of a secondary or symptomatic kind: when 
these signs of local disturbance are nut present, and yet the symptoms are evidently those of common fever, we are as much entitled to credit in our practice for seating it in the head, as we are for giving it the name of meningo-gastritis, pneumonia, catarrhal fever, \&c. \&c.

Let us now, in conclusion, call to mind, in an order as natural as we can, the general symptoms of this most common disorder, common, simple, or inflammatory fever. Should we perceive its approach, we shall generally discover that the horse first shows signs of dullness and heaviness, by hanging down his head, and manifesting more or less disinclination to move; this will probably be followed by symptoms of chilliness, such as staring of the coat, coldness of the surface of the body and extremities; and I have more than once seen an actual rigor or shivering fit. These symptoms may precede those essential to the constitution of the fever, but they are not commonly heard of, and in many cases do not take place at all. They are succeeded by a warm skin, a hot and dry mouth; a quick, full, and hard pulse; respiration perceptibly increased, but not remarkably so, the animal drawing his breath apparently with more weight or labor than celerity and pain; loss of appetite; costiveness; urinary discharges altered; general sensibility more or less increased. The case of ague related by M. DAMOISEAU is a very remarkable one, and bears so well upon this disputed point, that I see no other way of supporting a denial of the fact than that of doubting the existence of any idiopathic or essential fever, as M. GI RA R D has implicitly done, both in men and horses.

In regard to the causes of fever in horses, in the idiopathic form nothing seems to operate so generally in 
its production as change of temperature; it is however also occasioned by over-exertion, or any cause that may dispose to plethora of system. It would appear to be epizootic at certain seasons, and probably may be also sporadic; but we lack observations upon these points.

The indications of cure are those grounded upon the common principles of inflammation. Venesection is generally advisable: but we have seldom to bleed largely, and not often have to repeat it. Purgatives are the principal internal remedies. They may be succeeded by hellebore and diuretics. Nitre is a favorite nostrum of most horse-owners ; and W H 1т E has gulled them with gratuitous praise about antimony: the one, I need scarcely repeat, is a mild diuretic; the other, in WhITF's doses, is just as effectual as his squills, ammonia, and aniseeds are in the cure of broken wind.

It is very natural for one to ask, as Gira R D has done, by what morbid appearances we characterize fever. Certainly not, I should answer, by any that may be found in the stomach and bowels. Put the same question to any eminent human pathologists, whose minds are unbiased by any favorite theory on the subject, and a similar equivocal reply will be returned. Mr. BLAINE says, that he has never witnessed fatality ascribable to fever.) I have. I have seen several fatal cases-I have had horses brought to me after hunting with the most unequivocal symptoms of fever, and those horses have died, and no organic lesion whatever has been discernable after death: I know that many modern practitioners will say that these were cases of pneumonia, and GIRARD probably would have named 
them meningo-grastritis; though, in the cases to which I allude, neither the lung's nor the boinds bore nuarks of disease. These however are comparatively rare cases; generally speaking, fever 11 horses is a very mild and controllable malady; unless where it is continnally receiving fresh excitement from the source which gave it rise, as in the secondary form, and then it not infrequently resists remedy and ends in death. 


\section{LEC'TURE ISXXV.}

On Munge, and other Cuaneous Affeclions.

MANGE, a name we derive from the French demangeaison, is a cutaneous affection so well known in its confirmed stage that, had I not reason to believe it occasionally presents itself in a dubious form at an early period, any description of its characteristic appearances might probably be dispensed with. Inveterate mange is marked by bare white patches upon the skin, particularly upon those parts which are thinnest and most vascular and sensitive; from these bare places the cuticle, which has turned white, desquamates and peels off in branny scales, leaving the cutis underneath exposed, and in the first instance red, owing to a spot of extravasated blood; afterwards however its surface emits some morbid matter which exsiccates and scabs the spot over. The skin is arid and harsh to the feel, and often, particularly upon the side of the neck, is puckered into wrinkles. The parts remaining covered show more or less progress towards the same morbid condition: the hair is thin and staring upon them, it has an unnaturally dry rough feel, and in places may be plucked out in bunches with the 
greatest ease. The desquamated white cuticle, the red spot or scab, and the unresisting eradication of the hair, may be taken as the diagnostics of mange.

Mange in horses appears to be a disease of similar nature to mange in dogs and itch in man: at least, Professor Coleman says, he has often had reasons for suspecting that persons looking after mangy dogs have transferred the disease to horses; and Mr. Buaine informs us, that he has seen the itch taken from mangy horses more than once. The disease in all three instances appears to originate in little pimples; these form vesicles; and the vesicle bursts and leaves behind a small red spot: the pimples seem to exist in great numbers, more particularly in parts where the skin is thin, and to run one into another, so as to form, in the inveterate stages, broad irregular patches, which become covered with a dry, white, morbid cuticle: The roots of the hair would appear to be secondarily affected from the cutis; for the coat does not come off until mange has existed some time, and even then, the stubborn inherence of some hairs in the very middle of the mangy places is very remarkable. The parts commonly manged are the face, particularly around the eyelids and about the muzzle, the sides of the neck, the withers and shoulders, the armpits, and the thighs.

It seems that mange may be generated either from immediate excitement to the skin itself, or through the medium of that sympathetic influence which is known to exist between the skin and the organs of digestion. We have, it appears to me, an excellent illustration of this in the case of mange supervening upon poverty; a fact too notorious to be disputed, though there may be different ways of theorizing upon it. It may be said, in 


\section{On Mange, and other Cutaneous Affections.}

regard to man, that personal uncleanliness is almost always combined with poverty, and therefore ought to be taken into the account; this argument however cannot be pushed far in respect to horses and dogs, for poverty and uncleanliness in them by no means go consecutively together, and one frequently exists without the other. In the place of poverty, Mr. Bua r. F has put debitity: mange, he says, has three origins; "filth, debility, and contagion." Now, with all due deference to this respectable veterinarian, whose experience has probably been much greater than my omn, I really do not conceive that he is warranted in ranking debility among the causes of mange; for, if it were one, how numerous would be the cases where poverty had no place whatever? Nothing, I apprehend, can more debilitate horses than excessive work, various diseases, and the long-continued use of certain medicines, and vet this debility, which is as direct and extreme as it can be, is not succeeded by mange. But, turn a horse upon a common where the pasture will just serve to keep him alive, and it is probable that his poverty will be attended with mange; whereas, had the same animal been turned into good pasture, though all other circumstances were the same, the certainty is, that he would not have becone manged. It is this last fact that makes me sceptical about the production of mange from a dirty or filthy condition of the coat: one animal is just as likely to foul his skin as the other, and the one that grows poor breeds mange, while the other comes up fat and healthy. It is true, that poverty and filthiness often gro together; but in this and other instances they are dirtinguishable, and this I take to be the only light in which the subject can be fairly viewed. Ana- 
On Bange, and olher Cutuneous Affections. 409

logy may be brought in support of a contrary opinion; but, where facts can be adduced that bear directly upon the point in question, I am little disposed to consult evidence coning from any indirect quarter, however strong the light it may reflect. How poverty operates in the production of mange I do not pretend to know, further than that it seems to originate in some peculiar derangement in the organs of digestion; and that when produced it does not appear to be curable by internal remedies, though the rectification of that derangement, and consecutive improvement of the general health and condition of the animal, indisputably conduce to the restoration of the healthy state of the skin. It is so far a local disease therefore that it does not appear to be removable by constitutional means; but, I think we must admit that it may have, at least in horses and dogs, a constitutional origin.

There is no question, I believe, but that mange is the most contagious disease to which hores are obnoxions. Horses have been known to take it from being curried with the comb used about a mangy subject: the fluid included in the pustules most probably contains the infectious matter ; for it is absolutely neressary for contact, mediate or immediate, to take place before the disease can be communicated. My father, in my recollection, has had as many as two hundred horses manged in the Infirmary at Woolwich at one time: I do not mean to insinuate that these animals all caught the disense, but it appeared to us highly probable that contagion was principally operative in the mischief.

In the humatu suhject, as cvery one has heard, sulphur is the remedy fine itch: and the various experi- 
ments made by surgeons at different times appear to have gone so far to establish this fact that there would seem to be a something in sulphur that combats itch more effectually than any other application with which we are acquainted. Mercurial preparations will also cure itch, but they are not so certain in their operation as sulphur. This has led to a pretty general use of sulphur in mange; but, though I do not mean to call in question the success of those who employ it, I must. contend that it falls short in efficacy of what I am about to recommend: at the same time, those who are prepossessed in its favor will find the subjoined formula, I believe, as neat and convenient, and as curative as any of the sulphurous compositions*. Leaving sulphur, we have displayed to us' a vast variety of applications for mange: we have ointments, liniments, lotions, \&c. in abundance-choice enough even to satiate the nostrum-seeking disciples of W Hrte. FERON tells us, "that the application of blisters in the mange, if properly modified and judiciously applied, will be found a real specific in this distemper, but their application must sometimes be repeated more than once." Had he said cantharides properly modified and ap-

* R Sulphuris žvj.

Ol. Communis to j.

Terebinth. Vulgaris $3 \mathrm{ij}$. M.

R. Sulphuris క̄iv.

$$
\text { or this, }
$$

Veratri Rad. pulv. 3 ij.

Hydrarg. Oxy-mur. pulv, 3j.

Ol. Communis ff j. M.

The Sulphur Vivun, which is cheaper, will answer this purpose quite as well, I believe, as the Sublimed Sulphur. 
plied one might have been inclined to make trial of it; but a blister over the whole surface of the skin appears to me to be such a formidable remedy that I take it the animal had better remain nanged. If it be worth while to insert any of them here, I may as well give a liniment that was commonly used at the Veterinary College some years ago*: what the present remedy is I do not precisely know. After all however, and I can speak with confidence, because several hundreds of horses-perhaps I might say thousands, have experienced its efficacy, I know of no local application equal in effect to a mixture of Barbadoes tar and train oilt: it gently stimulates the surface, suppresses the morbid irritation, restores healthy action, and ultimately so supples and softens the skin that it acquires the feel of chamois leather, and the coat its former fineness and glossy aspect. If the season admit of it, a sunny day is to be preferred for inungation: the operation should be performed with a soft brush, with which the ointment ought to be well rubbed into the affected parts. A second inungation may be required at the expiration of a week, and, about the same interval, should the case be an inveterate one, probably a third, and even a fourth. Horses who are standing in the stable will be benefitted also by soiling and paying some attention to their digestive functions; but the best situation for mangy horses is the grass-field, where they may be turned out together, and even, I believe

* R Ol. Communis thj.

Spts. Terebinth. そ̌iij. Misce, deinde adde Hydrargyri Oxy-muriat. 3rs. solut. in Acid. Muriatic. 3ij.

+ Rx Petrolei ̧̌iv.

Ol. Communis ft.j. M. fiat liniment. tepid, applicand. 


\section{On Mange, and other Cutaneous Afjections.}

without risk, with sound horses, providing they are previously thoroughly anointed. Though it is not absolutely necessary, or invariably considered worth the trouble, to wash these subjects with soap and warm water before the dressing is renewed, I am of opinion that it is a part of the treatmeat that ought not to be disregarded when the weather is warm, unless the horse be at grass, or be about to change his coat, in which cases a single final ablution may be all that is required. Every article of saddlery and stable furniture which may have rubbed against his mangy surface, it will be requisite to soak and thoroughly cleanse before they be used for other horses; and the stall (together with its rack, manger, \&c. in which he stood in a state of disease) ought at least to be properly washed with soap and boiling water, if not fresh limed or painted.

Very often, horses are brought to us on account of rubbing various parts of the body, particularly the root of the tail, the mane, and perhaps the head and neck as well: the hair is ruffled and worn by the friction; indeed, sometimes the places are rubbed quite bare. The cutis however remains commonly unabraded, and the hair, though worn down by friction, still firmly adheres by its roots : to which may be added, there is no appearance of pustule, nor are there any red spots. These cases are generally referable to what is vulgarly called "heat of blood," or, in medical phraseology, to plethora and constitutional excitement; and therefore require some mild antiphlogistic course, such as a dose or two of purgative medicine, perhaps the abstraction of a small quantity of blood; with which may be recommended, increased exercise to produce occasional sweating, and bran mashes in licu of corn once or twice 
a day. I am in the habit of prescribing also for these subjects one of the subjoined lotions*, with which the parts rubbed should be sponged fiequently in the course of the day.

Now and then we see horses, particularly in the spring of the year, suddenly breaking out, over the body generally, into lumps or pimples : this eruption is called by our farriers a surfeit. Though commonly the attendant of full condition, it seems to arise from some mysterious derangement or other in the digestive operations; for it will often disappear as suddenly as it came on, in which respect it bears some analogy to urticaria or nettlerash in the human subject. A case of this description was treated by my father last summer which was no less remarkable for the number, size, and universality of the lumps, which were as large as common marbles, than for disappearing in the course of a few hours on one day, and breaking out afresh on the third or fourth day following; and this was repeated several times. The treatment for these cases consists, in bleeding, according to the apparent disposition to plethora; in exhibiting alterative or laxative medicines, alternated with mild doses of calomel, hydrarg. c̀ cretâ, or blue pill; and in directing waiking exercise for three or four hours in the course of the day.

\section{On Grease.}

Grease may be defined to be, an issue from the cutaneous pores of the heel of a peculiar, greasy, offensive matter; and may be said, invariably, to originate

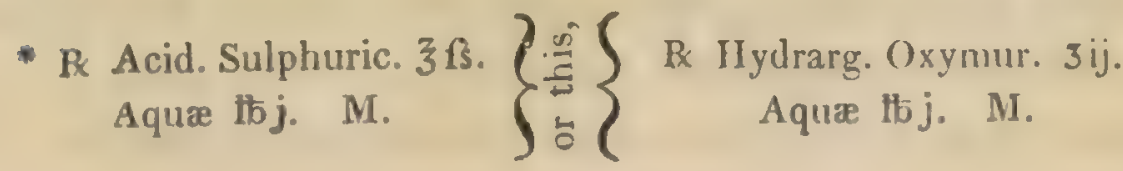


in inflanmation, or at least in increased vascular action.

The heel of the horse bears some analogy to the axilla of the human subject; it is furnished with cutaneous glands of a particular kind which supply a secretion of an oily or ceruminous nature, differing from any other in appearance and odor; and this secretion lubricates the skin, supples and softens it, and protects it from the irritation of dirt, and, to a certain degree, from the contact of moisture. Mr. BLAINE considers the inflammation, of which grease is the consequence, to be of a specific kind; but, looking into its symptoms, its progress, and the circumstances under which it makes its appearance, I really do not see the necessity of calling any specific influence into our ratio symtomatum. The peculiar nature of the morbid matter is what $\mathrm{Mr}$. BLAINE rests this hypothesis upon; but $I$ can see nothing more in the case myself than that, the structure of the part being peculiar, inflammation brings forth a secretion from it which in its natural as well as morbid condition is also peculiar. However, not to dilate on this point, one that admits of a variety of elucidations, grease, I repeat, originates in inflammation, and on the principles of simple phlegmon we shall find its phenomena and causes to be explicable, and its cure certain. Professor Coleman's theory on the production of grease used to be this-The heels being very remote parts from the heart, and the circulation in consequence proportionably weak in them, a congestion or stagnation of blood takes place within the capillaries, which those vessels have no other way of relieving themselves of but by pouring it forth in the form of grease. All I have to say about the actual condition of the capillaries 
myself, is, that, as the case resolves itself into one of inflammation, I should conceive that those vessels may at one time be in a state of increased, at another of diminished action, according to circumstances; that the tumefaction was rather a consequence of effusion than of distension; and that augmented secretion might result from increased action as well as from an oppressed or debilitated circulation. Professor PEALL draws a comparison between grease and chilblain in the human subject. "The disease is, in truth," he says, "the chilblain of the horse." So far that they are both local cutaneous inflammations in parts remote from the heart, and induced by vicissitudes of heat and cold, there certainly is an analogy; but grease differs from chilblain in that which constitutes its very essence, viz. in its characteristic sign being that of a peculiar greasy issue, nothing of which kind happens in chilblain : in fact, one is seated in a part of the skin furnished with a peculiar glandular apparatus; the other affects an ordinary part of the same tissue, simply because it is exposed, and in consequence subjected to a debilitated or disturbed circulation.

The etiology of grease throws considerable light upon its veritable nature. Horses who are at pasture or in strawyards, in situations, in fact, where heat and cold are not naturally, and cannot be made artificially, suddenly operative upon the heels, rarely have grease. Those that have grease in stables are mostly coach and cart horses, having thick fleshy heels, and white legs, who are very subject to get their heels wet and do not commonly have such pains bestowed upon them to dry their legs as hackneys, hunters, and racers have: in- 
deed, among the latter, grease is a very uncommon disease. Such horses also stand in stables hot and filthy from dung and urine, the very exhalations from the litter of which, ammoniacal and stimulating as they are known to be, may, as Professor PEalL plausibly argues, prove an additional excitement. Grease formerly made great ravages in the Cavalry and Ordnance service; whereas, at the present day, the disease is scarcely known. 'This change for the better is ascribable to three causes : to the proper ventilation of the stables, the greater attention paid to grooming, and to the presence of a veterinary surgeon who checks in the outset any such casual occurrence. I have heard Professor ColkMAN say, that that soldier deserves punishment whose horse has become greasy; and I perfectly agree with him-it is, as the cavalry are now stabled and shod in this country, in the power of every dragoon to preserve his horse free both from grease and from thrush.

Light-chesnut and white-legged horses have been remarked to be more particularly the subjects of this disorder where great numbers have been simultaneously affected. The autumnal and winter seasons, and cold and wet springs, are the times when grease prevails, and particularly in frosty weather when snow is present: great numbers of cavalry horses have been attacked on return from camp. The condition of system under the influence of which horses change their coats is said to favor its production. The hind legs are the parts commonly greased; it is seldom found in the fore legs: the rationale of which is, that the hind heels are further removed from the heart, ergo, more predisposed to 
inflanuation; and, at the sante tine, are more exposed to the vicissitudes of heat and cold, from their liability to get wet, and the little comparative pains taken to dry them afterwards, ergo, have the exciting causes applied in a more forcible degree. The same principle of reasoning applies to horses who are lightchesnut-colored, have white leg's, or are changing their coats. By no means an infrequent cause of grease, when proper management is wunting, is a blister to the hind leg; and, if any thing goes to prove the simple or common nature, as well as the locality of the inflammation, it appears to me to be this; Mr. BLArNe however admits this most fully, and yet he contends for the specific nature of the disease: I cannot, I must acknowledge, reconcile all this; analogy, which one might be apt here to resort to, turns decidedly against Mr. Btarve's pathology.

SAINBEL, who wrote "An Essay un Grease," for which he was presented with a prize by the Royal Suciety of Medicine in France, thus commences his paper - The grease is in general a cutaneous chronic alfection, sometimes inflaminatory, sometimes infectious; and I have known it cuntagious: it invades the legs of horses, asses, and mules; but seldom attacks those of the ruminating species." We are told that cow-pock had its origin in the transfer of the matter of grease from the heel of the horse to the teat of the cow, and that the disease may be conmunicated to the human subject by inoculation with this matter, the same as with that taken from the ulcerated teat of the cow; some have gone further than this, and said that glanders and farcy could be generated in this way: the accounts of these strange transactions however have made but lit-

PART III. 
tle impression, for we hear nothing of them nowadays, and this is not a very bad criterion of their want of truth and foundation altogether. I have heard Professor COLEMAN say, that there never was a well authenticated case of cow-pock being produced from grease ; and, I verily believe myself (though I do not know that the fact has been satisfactorily experimentalized on) that there is no truth of its being communicable among horses. In certain seasons and situations the disease is certainly sporadic; but, then, the causes are too manifestly operating among horses under the same circumstances to refer its production to infection or contagion.

The symptoms of grease, in its simplest form, are-an exudation of a greasy, offensive matter from the skin covering and surrounding the heel, attended with preternatural heat, redness, tenderness, and occasionally general tumor of the heel and fetlock. Soreness or lameness on first leaving the stable is commonly perceptible; though it is seldom in sufficient degree to call attention to the disorder, the first intelligence of which is commonly communicated by the groom. And here I may remark, that the presence of grease is a pretty infallible test of negligent grooming; for, as may be inferred from this exposition of its nature and causes, a horse whose heels are kept dry and clean, and never suffered to grow dry of themselves after having become wet, will be in little danger of contracting grease. Should the diseased action not be corrected, the inflammation extends up the leg, which in consequence takes to swell, and grows warm, and tender on pressure; so great indeed is the morbid sensibility of the heel often in this stage that if the part be but touched the animal instantly catches his foot off the ground, evincing 
considerable pain. The greasy issue, by this time augmented in quantity, stands in large drops among the hairs, which grow strong and show an inclination around the point of the heel to become bristly. No affection causes more pain or soreness on motion than grease, the heel itself being so very moveable and sensitive a part; when both hind legs are violently affected, the animal is often so lame that when first taken out he can but with great awkwardness (evidently from excessive soreness felt, particularly in extending the heel) even walk at all; after he has walked for some time however, this soreness diminishes greatly or entirely leaves him, and he will trot with much apparent freedom : this accounts for the many greasy horses we see in constant use. We may explain this relief, by saying, that exercise has produced some absorption, and this has taken off the distention and consequent tenderness and inability to the free motion of the joint, and has moreover provoked a copious secretion which has abundantly lubricated and softened the skin of the heel, and thereby further diminished its morbid sensibility. As this does not therefore incapacitate the animal from working, the case is sometimes neglected and becomes inveterate grease. Pustules arise in various places, mostly around the upper and back part of the heel, in consequence of the irritation occasioned by the discharge; these burst and expose a raw surface from which granulations arise in clusters resembling (as SAINBEL happily expresses it) " the outward coat of a pine-apple:" these pustular excrescences are called by our farriers grapes. The morbid action of the skin continuing unchecked, grapes, which are nothing more in 
their origin than granulations springing from an inflamed surface, shoot out into irregular spherical forms and at the same time swell considerably, so that many of them (though they vary greatly in size) grow as large as serments of marbles; in the course of time they become of a schirrous or rather a cartilaginous texture, and 1 have found them assuming the nature of horn, which would appear to be their natural tendency or ultimate change. The skin itself all this time is gradually altering its texture: it becomes morbidly thickened, and assumes a cartilaginous toughness and hardness in accordance with the grapy excrescences; I have got a preparation before me in which it is thrice its natural thickness, but I have never met with it horny.

Altogether, the heel in this condition puts on the appearance, were it not for the color, of a full-blown cauliflower: and this seems to be the maximum of virulence. In the mean time, also, the hair has gradually fallen off, so that the grapes are either quite bare or very thinly bestrewed with hairs which stand out after the form of bristles from a hog's back. A purulent greasy discharge, here and there tinged with blood, issues from the skin not yet covered or altered by grapes, the fetor of which is intolerably noisome; at the same time, there is something in the odor of it which is peculiarly characteristic of the disease. Inveterate grapes are intensely reddened from their high degree of vascularity, and are exquisitely sensitive, giving great pain when touched or pressed. They commonly sprout from the upper and back part of the heel; rarely from the coronet, (to which the disease extends occasionally,) but very often from the hollow of the heel; though it frequently hap- 
pens that they spread up the hind part of the leg, they rarely reach the hock. The leg acquires in some cases an enormous bulk, so that the limb from extreme soreness as well as swelling, is considerably pained and inconvenienced in action: in fact, the part altogether, to use SAIN BEL's translated expression, is " a cumbersome mass of disease;" and, I may add, a most ubnoxious member from the rancid oily issue which is continually running from the crevices between the grapes, mingled with the purulent bloody matter discharged from the ulcerated surfaces of the grapes themselves.

The treutment will be very simple, or be more or less complicated, according to the duration, stage, and virulence of the disease. Professor Colmuan, in lecturing on this part of our subject, has always shown more than ordinary solicitude in inculcating the doctrine, that, as wet or moisture is the common cause of grease so in any form it will, generally speaking, prove injurious in the treatment. This appears to me however to admit of such qualifications as will go al most or quite to undermine the general assertion. In the first place, we certainly are not warranted in laying it down as an axiom in medicine that what by predisposition gives rise to a malady is not to be enployed as a remedy, else why are snow and ice-water recommended as the best remedies for chilblains?cold affusion in fever, pneumonia, \&c.? for, I must remind you here, that moisture is not the exciting, but the predisposing cause of grease: the disease would not follow its application at all unless the heels had re-action excited in them from other causes. And in the next place, even this wet or moisture under circumstances the most favorable to the production of 
grease, viz. in a hot stable, would not in all probability give rise to any such ill consequences were the circulation kept up until the parts became dry again, instead of suffering it to be rendered weak and tardy by the cold resulting from evaporation : a principle which, once understood, will direct us in the proper use of wet applications for grease, should practice be found to give them the preference.

Let us now proceed to give the remedies; and, in so doing, endeavour to divest the subject of treatment of that complexity in which our best authors seem to me to have obscured it by paying more attention to one another than to the simple dictates of practice. For the sake of simplification, let us put down our cases summarily, and then prescribe for them.

Should the case be a mild and recent one, augmented issue be the only ailment, some simple astringent application will be all that is required*. A powder is generally employed, after which I myself prefer the form of ointment $\S$, and for this reason : that, as the astringent powder has a tendency to suppress the secretion, the discharge will, sooner or later, become arrested altogether, at which time, the heels

Ii Pulv. Aluminis

_-Bol. Armen. àā p.æq. M.

R Pulv. Carb. Ligni

$$
\text { or this, }
$$

- Calaminæ ì p. æẹ. M.

Tr l'ulv. Aluminis $\Xi \mathrm{ij}$.

Ungt. Simplicis $3 \mathrm{j}$. M.

or this,

R Thent. Citrini

— Simplicio H. xy. M. 


\section{On Grease.}

being dry, the skin grows hard and contracted and disposed to crack, all which, I conceive, is in a great measure prevented by the unguent artificially smeared over the surface. Though I ordinarily use the powder, therefore, in the first instance, I substitute some mild astringent unguent as soon as the discharge has become suppressed. Should the legs be vedematous, walking exercise twice a day in a dry and clean place will be beneficial, and a diuretic ball may be administered every other night.

But, in a case in which the heels are hot and tumefied and tender-in other words, in which inflammation is very active, I have no hesitation in saying that we ought to mitigate it in the first instance by assuasive means before we think of employing astringents. Some recommend fomentations, but they are inconvenient applications and possess no advantage over a common poultice. And of all poultices the bran poultice, from an absorbent property it possesses, is one of the best. Where there is much fetid discharge however, and a degree of malignancy about the case, a poultice of charcoal is to be preferred, one that possesses still greater astringency and is commonly attended with the best consequences. At the same time purgation should be put in practice, low diet, and even in some cases the abstraction of a little blood from the thigh will be advisable. As soon as the inflammation has subsided, astringents may be employed; they will require however to be stronger*

* Re Cupri Sulphat. 3ij.

Aluminis 313 .

Bol. Armen. 3j. N. 
than those used for slight cases, and (what is very important to remember) they ought, not merely to be smeared over the heels, but spread upon pledgets of tow and kept applied, by means of bandages, for two, three, and even four days together, without taking the horse ont of the stable; during which time it is a part of my practice to give a strong dose of purgative medicine, should that not have been done before: at all events, the bowels should be kept moved. On the third or fourth day, when the bandages come to be removed, the legs will cominonly be found much swollen, but the heels very considerably improved. It will then depend upon their condition whether the bandaging and liniment are to be renewed, after the horse has been walked out for at least two hours, or whether the cure be thought sufficiently advanced to be completed with mild astringent applications, powders and subsequently ointments. Also give, from time to time, diuretic balls. Rowcls are not adapted to do much good in grease. It is very important that due attention be paid to exercise. After having been under the operation of the bandaged dressings for two or three days, the animal will require, for at least two hours, to be walked

Et contere in pulv, subtiliss. deinde arde

Ol. Commun. ३iij.

Lt frat linimentum.

R Cupri sulphat.

or this,

Calaminæ āà §̧j.

In pulv, redact. et misce cum

Ol. Commun. ऊiv.

Ur, the Citrine ointment, made thiu witl: wh, may he used. 
ibout, prior to any repetition of the dressings; or, should it be a case not requiring bandages, exercise for one hour ought to be given both in the morning and afternoon. It is also of some importance that the animal stand in a clean and ventilated stable; and if it be large enough for him to be let loose in, that is by far the most suitable.

In consequence of grease being comparatively a rare disease 10 what it was twenty years ago, and from the improved state and diffusion of the veterinary art, it is by no means common nowadays to meet with an inveterate case of chronic malignant grease; and, indeed, such cases are not to be desired, for they are vastly troublesome and tedious to treat, and in the hands of timid and inexperienced practitioners are not recoverable at all. In the year 1821 a case of such duration, virulence, and malignancy was sent to my father to treat, which, in the end he completely restored, that I rlo not think I can draw up a more systematic and efficacious plan of treatment than the perusal of this case affords; I shall therefore relate it.

June 19th, 1821. Admitted a chesnut mare, seven years old, rather low in condition, who has had grease for these last two years. The disease originated in the ordinary way, and has arrived at its present height and malignancy from neglect, continued exertion of the parts, and occasional mal-treatment. All four heels, but particularly the hind, are studded with prominent grapes, thinly clad with bristled hairs, through which their bare surfaces appear intensely reddened, and here and there are issuing blood. In places where there are no grapes the skin exhibits a 
red, rough, elephantiasical aspect. The grapes extend for several inches above the fetlock; but.they are all situated at the posterior parts of the legs. The hollows of the heels are entirely covered with ulcerated grapes. A greasy and extremely offensive matter issues from every part of the morbid surface, which lodges in the crevices between the grapes as if so much oil had been poured thereon. On the same afternoon on which she was admitted, my father submitted her to the following painful operation. Having properly secured her standing, with a sharp scalpel he commenced paring off the grapes from the hind heels, upon a level with the surface of the skin, in doing which he found it necessary to have firing irons near" him to staunch the hemorrhage as he proceeded, which, owing to the high vascularity of these excrescences, was very considerable; the cautery however was prepared to be used with another object, that of destroying the morbid surface from which the grapes had so long been growing. All the larger grapes having been in this manner removed, (which proved to be cartilaginous, some even horny, in their composition) the remainder of the diseased surface was lightly seared over, and the mare was returned to the stable, and dressed with the following liniment, which was spread upon pledgets of tow, and they were confined by many-tailed bandages :-

R Cupri Sulphat. in pulv. 3 j.

Aluminis 3iv.

Ol Communis to ij. M. pro Linimento.

Both the hind and fore heels (although the latter had not been operated on) were dressed with this; and a brisk purge was given afterwards. 
24th. The diseased parts were covered over with sloughs; these being wiped off, the surface presented a raw and highly vascular appearance, and now showed distinctly the smaller grapes that had been left unpared. She was cast, the fore legs were operated upon as the hind ones had been, and the hind legs were also rid by the knife and cautery of many more grapes, which before were hidden by larger and more prominent excrescences. She was afterwards dressed as on the first occasion; and some of the Unguent. Ferri Acetatis was applied to her frogs, in which thrushes had made their appearance since last dressing

27 th. Since the 24th some little fever has been hanging about her; her appetite has been impaired, she is tucked up, and has evidently suffered from pain. She was walked out, and afterwards dressed as before.

30th. A raw surface pretty free from grapy excrescences now presents itself. Her legs were simply smeared over, without any pledgets or bandages, with the ointment, and (the weather being warm) she was afterwards turned into a grass paddock.

July 4th. An ichorous discharge, but which is not very offensive, issues from the ulcerated surfaces. Ointment repeated.

9th. The surface looking florid and healthy. What grapes still remained unpruned were to-day touched, by means of a feather, with nitric acid. They instantly turned yellow, and a process of solution began in them. The surface generally was washed with Sol. Cupri Sulphatis-3j.-ad 3j.

11th. Sloughs caused by the acid, turning out, and leaving deep cxcavations. Blue solution repeated. 
15th. The heels have begun to granulate, and in places are showing signs of cicatrization. Unguentum Aluminis.

$30 \mathrm{th}$. Since the $15 \mathrm{th}$ the dressings have been mild, and such as are promotive of the granulating process. The heels are making great progress towards health.

After this, nothing worth mentioning was done. Not only a new surface, but in most places a new skin, was eventually formed, and the mare was ultimately restored to her master perfectly sound, with some trifling induration of the parts which had so long been the seat of this noisome disease. 


\section{LECIURE LXXVI.}

\section{On Staggers.}

THIS is a generic name for affections of the brain, taking its origin from a common and prominent symptom denoting them, that of the horse reeling or staggering in his attempts to walk. The brain is an organ much less exercised in the brute creation than in man: the latter derives from it the various passions and intellectual faculties comprehended under the term mind; the former has simply superadded, by it, over and above common sensibility, instinct and reason enough to provide himself with food, avoid danger, and seek sexual intercourse. As might be inferred from this diminished importance and use, the organ in animals is considerably less subject to disease.

In horses, the brain may be actually or primarily diseased, or it may be sympathetically or secondarily affected; and these affections will, in certain stages, give rise to the same symptoms; so that, 'were it not for other concomitant circumstances, we should be unable to distinguish them. I shall first show how the organ is sympathetically affected.

No fact is more notorious in physiology than the sym- 
pathy constantly operating between the head and the stomach : a violent blow upon the head makes a man sick at stomach, and disordered digestion is the common cause of head-ache. What disorders the human stomach, generally speaking, is food improper in quantity or quality, or taken at improper intervals. Now, let us apply these few simple and general facts to horse pathology. Horses who are kept long fasting and then have provender spread before them in profusion, will be likely to glut themselves until they have crammed their stomachs with food and an uneasy sensation is created which deprives them of any appetite for more. This uneasiness is manifested by the following symptoms. The animal becomes dull and drowsy, and grows insensible to what is passing around him; he hangs his head under the manger, and loathes what food remains before him; the drowsiness and insensibility increasing, he rams his forehead against the rack or any resisting body before him, stands fixed in that position, and falls into a state approaching lethargy : this last is the most remarkable, and may be regarded as the diagnostic symptom. The pulse is either unaltered, or it is slower than in health-about 40. He labours somewhat in drawing his breath, though his respiration in this stage is not commonly accelerated. If he be suddenly roused however, both the pulse and respiration will be momentarily hurried. When led out, he walks unsteadily or staggers about. His extreme parts are cold; his bowels obstinately constipated; and, according to Mr. BLAINE, who sets it down as a constant and invariable symptom, though I must confess I have overlooked it myself, there appears a yellowness of the mucous membranes of the nose, eyes, mouth, and rectum. 


$$
\text { On Staggers. }
$$

Unless he be relieved from this lethargic and insensible state, he is gradually aroused from it by symptoms of inflammation of the brain, which commonly make their appearance in a more or less marked degree before death; but the delirium does not mount to that height which it does in what is called, in contra-distinction to this, which has received the epithet of sleepy, mad staggers.

Professor Colfman considers that this disorder takes its rise from over-distention of the stomach. He was once consulted about some horses among whom there had been a strange and unaccountable fatality, and he found that they were in the habit of continuing at work for ten hours together during the day, and on their return home at night were abundantly supplied with food. The Professor, with his wonted penetration, soon discovered the source of the evil; and ordered, for the future, that the horses should be fed in the middle of the day also, by means of nose-bags ; which salutary practice put flight to the epizootic. Now, this is certainly a strong case to show the stomachic origin of the malady; and yet I cannot refrain from entertaining doubts that, after all, over-distention is the exciting or efficient cause. Else, how comes it that gastric tympany * does not likewise produce staggers? The stomach is quite as much, nay, more distended in one instance by air than it is in the other by food: for I have seen two cases in which it has burst, and in another the organ was full thrice its ordinary volume. If mechanical distention be the cause, surely here it was in full force, and ought to have been effec-

* Vide Part II. Lecture xliv. page 424 , in a note. 
tive! Mr. Coknkux V. S. Rryal Artillery, has lately done me the favor to communicate to me from Ireland an account of two fatal cases, both of which succeeded voracious feeding after long-continued hunger: in one of them, the stomach with its contents weighed $45 \mathrm{lbs}$., the other wanted only a few ounces of 50 lbs.; they were both enomously distended. But, if distention be not the exciting cause of the cerebral derangement, what is it? I cannot divine myself, unless it be the presence of such a load of raw indigested matters in the stomach, whereby its functions are deranged, and its surface for want of secretion probably irritated by them; for Mr. BLAINE tells us, that in every case that he has examined, there has been "an inflamed state of the depending portion of the great curvature of the stomach towards its pyloric orifice," differing from any other inflammation of the part-one sui generis, and he has therefore named the disorder, "SPECIFIC INFLAMMATION OF THE STOMACH." I must protest myself that I have a great aversion to the epithet specific as it is commonly employed in medicine, because it seems to interdict all further inquiry, and yet does not, after all, help us out of our difficulties; and it is for this reason that I do not consider the point worth contending until we come to discover in what this specific inflammation differs from that which is common in its nature. What is called the hove in cattle is commonly mentioned here by way of illustrating this part of pathology; but, I should say that the hove was a tympanitic affection, that air and not food stretched the paunch, and it strikes me that the symptoms are not those expressive of pain in the head, but of pain in the belly: could the head here be proved to 
be affected, it would certainly operate in favor of the theory of distention.

The treatment of this disease has been notoriously unsuccessful: Mr. Blaine says, "I never witnessed a successful issue." It may be deemed arrogant in me to attempt to show cause for this universal failure, but I cannot help thinking but that we have been strangely erring in pointing out remedies for this malady. Of what avail can we expect our aloes and our oil, \&c. to be, introduced into a stomach, as they are, already crammed to distention with indigested food? Phlebotomy certainly will tend to relieve any congestion or inflammatory action there may exist in the brain; but, the main question for us to consider, is, whether it can have any effect in unloading the stomach. Practitioners are in the habit of expressing their surprise at the obstinate apathy of the bowels; whereas, is it reasonable to suppose that 12 or even 20 drams of aloes can have any effect when they can pass no further than the stomach? Assuredly, in some few cases it is possible that active stimulants may make such an impression upon the stomach as to excite contraction in it; in the generality however, the atony consequent upon the over-distention seems to render that hope even but a forlorn one. It is perhaps more easy to expose the inefficacy of such means as these than to point out measures likely to prove effective in removing the cause of the evil. Now that we have got tubular probangs, and can without much difficulty pass them into the stomach, in my opinion it would be advisable so to do, and inject through the tube a quantity of any tepid innoxious fluid, and thus cudeavour to evacuate the contents of the stomach by copious dilution: the insensible state in
PAIT III.
$2 \mathrm{~F}$ 
which the animal is at the time, is very favorable for the experiment; and it is one that I shall certainly put into practice the first opportunity I may have. This should be followed by a potent purgative in a fluid form, combined with some stimulant*, and a detraction of blood, large in proportion to the apparent signs of congestion in the brain, or gastric inflammation, and to the duration of the disorder. These evacuations should be followed up by blisters to the head, and over the region of the stomach; and the purgation may be promoted by enemæ. After this, hellebore will be found particularly serviceable in abating any impulse of blood to the head, combined with aloes or calomel as often as the bowels appear to require their action renewing.

What is vulgarly called mad staggers is neither more nor less than

INFLAMMATION OF THE BRAIN-phrenitis, as it is teohnically named. BRACKEN considered it as an apoplectic affection; but the lethargic stage is not marked by that suspension of sensation and voluntary motion, and that profound sleep, which characterize apoplexy, and we know from experience that this stage is only the prelude to the phrenetic paroxysm which constitutes the veritable mad staggers. Mr. BuAINE, I conceive, has very properly refused to adopt that appellation, and has substituted for it phrenitis.

It appears that what we call sleepy staggers may denote disorder either of the stomach or head; but, that

* R Decoct. Aloës.

Tinct. Cardamomi vel Zingiberis āā tôj. $\mathbf{M}$.

Fiat haust. statim exhibend et repetend. p. r. n, or the tinctura crotonis may be substituted for the decoct. aloës. 
the stomach-affection never gives rise to mad staggers. Now, as the mad staggers is commonly ushered in by the lethargic or sleepy symptoms, it is a point of vital importance in practice to be able to distinguish between these affections, in order that we may know for certain whether our remedies must be directed to the head or to the stomach. Mr. BLAINe gives us as diagnostics, the yellowness of the eyes and mouth, and "spasmodic twitchings" of the skin, which, he says, eminently characterize disorder of the stomach : I cannot charge my memory at the present moment with these characteristics; but, I would add, that here, as in many other disorders, the circumstances of the case ought to form, in my opinion, a very prominent feature in the diagnosis. The horse may be attacked at grass, as well as in the stable, or there may have been a previous long abstinence, or food of a luxuriant nature may have been supplied in improper abundance, all which circumstances would lead us to refer the malady to the stomach. On the other hand, an evident disposition to plethora, an attack immediately following excessive exertion, or continued exposure to a meridian sun, would induce us to look to the head.

There are particular states or affections of the brain in which the animal manifests the same somnolency and lethargy as characterize disorder of the stomach : horses so affected will stand, commonly with their heads thrust against some hard body, with their eyes closed, and doze or sleep until they actually fall down on a sudden, often backward, in the stall; this, suddenly awaking them, startles and alarms them greatly, they hastily get up, but soon relapse into their former stupol. 
There is in the eye of such horses a remarkable stare -a fulness and stupor that seem to express a sense of weight in the head, which is hung down under the manger in a state of extreme dejection. The pulse at this time is often not more than $40^{\circ}$; never, I believe, does it exceed its ordinary frequency, unless anything happens to rouse him, such as the shutting of a door or suddenly approaching him, and then it instantaneously rises into extreme quickness, from which it as suddenly sinks again into its former tardiness. In some cases, the breathing is stertorous. His appetite a!l this time is not so much impaired as one would expect : place hay or corn before him, and rouse his attention to it, and he will probably commence feeding; and yet, such is occasionally the irresistible somnolency that I have seen the animal fall into a doze literally with the hay in his mouth. The bowels participate in this torpor, and are obstinately constipated. These lethargic symptoms may continue for several days, and at length end fatally; or they may be succeeded by the phrenetic paroxysm, which is still more likely to prove fatal. The symptoms are those of wild and furious delirium. The first warning we have of its approach may be commonly gathered from the rising of the pulse, acceleration of the breathing, and the renewed lively aspect of the countenance. The eyes become animated, have a vacant and alarming stare, and the pupils dilated; the conjunctiva is flushed; the nostrils are erected, their lining membrane highly reddened, and the breathing grows quick and painful. The pulse becomes full and strong, and very irregular; at one time it is very quick, at another it intermits; or now it 
is hurried, now it sinks again. Fits of delirium seize him from time to time: he furiously dashes himself about, throws himself down, lies in a state of temporary insensibility, his eyes appearing as if ready to start from their orbits, his pupils dilated, glassy, and insensible; suddenly he rises from this comatose state, and either becomes again convulsed with phrensy and pain, or relapses into stupor and somnolency, from which he is only roused by another fit of delirium. Approach to such an animal is attended with considerable danger; for, now and then, while attempts are making to administer medicine to him, he will throw his head up, be suddenly seized with vertigo, rear up, reel round upon his hind legs, and precipitate backward with such dreadful force that those in attendance conclude every such fall must prove his destruction. As he grows worse, instead of lying in a state of insensibility, after the fall, he will pant and perspire and perhaps foanı at the mouth with pain; convulsions seize him from time to time, and he will often survive in this dreadful agrony for many hours, unable or unwilling to rise again, until death put an end to sufferings which defy all human interference.

The state of the brain in staggers, generally speaking, appears to be, in the beginning, that of simple congestion or unequal distribution of blood; this, if it be not timely diverted, runs into actual inflammation, of which many and various are the consecutive effects : the first of these morbid states would appear to be denoted by the stupor and somnolency present; the approach of the last seems to be manifested by the rising of the pulse and the indications of delirium. In most of the heads I have examined, I have found vascular 
distention of the membranes, minute bloody specks upon the divided surfaces of the cerebrum, turgidity of the plexus choroides, and more water than natural within the lateral ventricles. In one instance I could detect nothing unusual in the brain but a remarkable yellowness of the substance of the cerebellum: this horse also had considerable disease of the liver; the gland was very dark colored, nearly black in places, and its texture had become so soft that the finger easily made way through its substance. Professor ColEMAN relates one case in his lectures in which no other morbid appearance could be discovered but an intense inflammation, approaching gangrene, of the Schneiderian membrane, not only throughout the cavities of the nostrils, but also extending over the frontal and maxillary sinuses; and another case, in which a tumor was found within one of the lateral ventricles.

Soon after my father entered the service of the Ordnance, at Woolwich, it became the custom there to turn horses, who had become low in condition and stale upon their legs from work, into the marshes to recruit their strength. During the months of July, August, and September, no case was more common than an attack of staggers among these horses; which my father attributed to the luxuriant pasture they were suddenly turned into, (which invariably loaded them with fat and consequently plethorised their systems,) combined with the dependent posture of the head, and the sultry heat they were exposed to in marshes destitute of places for shade: this practical observation made on a large scale throws considerable light upon what I have advanced on the etiology of staggers.

The horses above alluded to were speedily relieved, 
providing they were timely attended to, by the free and immediate use of the lancet: indeed, every common farrier knows that you must bleed freely in staggers, but there are none of them, I will venture to say, and but few veterinarians, I am apprehensive, who carry phlebotomy to the extent they might and ought. One would think, from our knowledge of the distribution of bloodvessels, that we could not draw blood from a more favorable source than the jugular vein; it is my duty however to remark here, that some practitioners give the decided preference to the temporal artery. In a late communication from my friend Mr. Cordeaux, he informs me, "that he has seen eminently good effects from bleeding from the temporal artery in six or seven instances; and that two cases, which were considered as hopeless, after several ineffectual bleedings from the jugulars, recovered, he verily believes, from an accident that befel them during the night, by which the pins were torn from their temples and considerable quantities of blood escaped and were found next morning in the manger." This last remark of Mr. CordeAux's corroborates the advice I have given of taking care to bleed profusely: most of these subjects will bear to lose six or eight, and some even ten quarts of blood. In my own practice however, in cases of urgency, in which congestion has existed for some considerable time, and the circulation has become tardy and oppressed, I have at times found the jugular veins to be the only sources from which I could draw blood in any quantity, a circumstance that invariably renders the prognosis highly unfavorable. The next consideration is purgation. The common saying is, "purge a horse with 
staggers and you cure him;" and there is much truth in the observation, for we know that hardly any one thinks of purging before he has bled the animal, so that the purge is acting under the most favorable circumstances. I would give $5^{f s}$. or $\ni i j$. of the pulvis crotonis in combination with the same quantity of calomel, and I should prefer the form of a drink to that of bolus. The decoction of aloes however is little or not inferior, that I am aware of. Promote their action by aloetic enemæ, taking special care that the rectum is first cleared of its contents by manual operation. I would follow up the first dose of purgative medicine by diminished doses exhibited every twelve hours, until copious purgation was established: there is little danger of super-purgation under such circumstances. In the intervals, between the repetition of the purges, hellebore should be administered every six hours in doses of 3 fs. or even $3 \mathrm{j}$. providing the first quantity make no impression: nothing, bleeding excepted, operates more effectually in diminishing the force and celerity of the current of blood to the head than the excitement of nausea at stomach. Having by these means sensibly weakened the impulse of the carotid circulation, the head should be shorn and blisters applied over the forehead, occiput, and temples; and they should be renewed every six hours until vesication be abundantly produced. The legs are generally very cold in the lethargic stage, and then there can be no objection to stimulation of them by applications of turpentine, nuustard, cantharides, or simply hot water; but I should not pursue this practice, did they remain cold, in the phrenetic stagres: I prefer simpler means, such as friction, \&c. which may be practised 
without fear of increasing the vascular excitement. We are recommended by some to insert a rowel under the jaw: rowels and setons too are not likely to be productive of harm in the acute stage of phrenitis, for neither of them will take any effect unless the pulse sink; but, if we would have recourse at once to effectual measures, these harmless means may be postponed until the violence of the inflammation has been subdued.

\section{On Strungles.}

"THE strangles," says Gi Bson, "has been compared by the French and other foreign writers to the smallpox in men;" because to both the young are more incident, and because the strangles " never seizes horses but once. But however that may be, it is certain the strangles is a critical swelling, which when it breaks and imposthumates, discharges somewhat obnoxious to the constitutions of young horses, by which they are usually rendered more healthful than they were before." BRACKEN, commenting on this comparison, (of Soleysel's,) satirically remarks, "that, on strict scrutiny, it will be found only flourishing upon the matter, as is too commonly the practice with longwinded authors. For, the strangles are produced from catching cold, or from what we term perspiration obstructed." TAPLIN, who lashes the pathology of Soleysel and Grison, in his usual unsparing style, tells us that strangles consists in the elimination from the system of an "accumulation of impurities" imbibed with the food in colthood; and that this "lurking viscidity is roused from its latent communication with the juices, and called into action by 
bringing the frame into sudden exertions and constant exercise,"-for, experience demonstrates, he continues, "that twenty horses have this distemper after being taken to work, to every one attacked with it" in colthood. WHITE manifests unpardonable ignorance of the nature of strangles when he begins his description with, "This disease consists in an inflammation of the membrane of the throat and nose, and swelling of the glands under the throat." Professor PEALL leaves the question of its nature and tendency unagitated. Mr. BLAIN $\mathrm{B}$ is the only writer, I have perused, who has offered any thing in the shape of theory consistent with the present state of our pathology. He says, independently of its attacking horses about the adult period, and only once, "it may be characterised as a catarrhal affection, accompanied with a specific phlegmonous inflammation of the cellular substance in and around the parotid and submaxillary glands, tending in most instances to abscess."

About the adult period of life, between the fourth and fifth years, and more particularly at the season when catarrhal and pneumonic affections are most prevalent, horses in general become the subjects of a diffused swelling under the jaw which in farriers' cant is called strangles. This tumor consists in a circumscribed inflammation, having all the characters of simple phlegmon, attacking the subcutaneous cellular substance included between the branches of the jaw, which in consequence becomes gradually filled and distended with effusions of lymphy and serous matters, acquires a firm and solid feel, tenderness on pressure, and a sense of unnatural heat. This commonly procecds to suppuration, ending, to all appearances, in a 


\section{On Strangles.}

common submaxillary abscess. Now this, and this alone, constitutes strangles: there are in books a variety of other symptoms described, but they are all concomitant or accidental, none, but this, being, properly speaking, essential to its existence. The usual concomitants are-membranous inflammation, giving rise to soreness about the throat, reddening and discharge at the nose, and perhaps cough ; tumefaction of the salivary glands, producing pain and difficulty in deglutition; and, lastly, some slight febrile commotion of system. Cynanche trachealis, pneumonia, and, some say, glanders, may supervene upon strangles.

By those who consider it necessary to expatiate upon the diagnosis of strangles and glanders, the diffusive nature of the submaxillary swelling in one, and its circumscribed limits and adhesion to the bone in the other, are generally laid much stress upon; but, really, this of itself is a very insufficient and even a dangerous guide; for, I have known many cases in which strangles has approached so gradually and insidiously, appearing to begin in swelling of the submaxillary salivary glands, that upon this naked and unnatural distinction the case might have been pronounced to be glanders. The true and safe diagnosis, so far as relates abstractedly to the submaxillary tumor, is to wait a while, and the case will soon manifest itself, if it be strangles.

To what this affection, one to which horses appear to be by nature subject at one time or other of their life, is attributable, I will not pretend to conjecture; and I acknowledge myself equally in the dark in regard to what or any salubrious change it may effect 
in the system. All I know about its specific character consists in the universality of its attack, the age of the subject, and the slight accompanying febrile derangement. Even in these-its peculiar characters, however, it manifests great irregularity: BRACKEN says, "although I have bred several colts myself, not one to my remembrance ever had the strangles, and this I attributed to their being kept well and warm in winter." A colt bred by my father had the disease at six months' old; and Mr. Coward informs me, that he has seen the disease three months after birth. In some cases, the attendant fever is so slight that it is hardly discoverable; and in others there does not appear to be any fever at all. So that, I repeat, even the peculiarities, or specific characters of this disease, discover much irregularity.

TAPLIN says, in concluding his account of strangles, "it is necesary to be remarked, and well remembered, that the disease we are now treating of, by neglect and inattention, sometimes degenerates into a confirmed glanders;" and this, I believe, still to be the prevailing opinion: the inquiry would involve us in the latter subject, and therefore I shall postpone it. It is now quite time, I think, that the notions of infection or contagion being a part of the nature of strangles were dropped: at least, everyday occurrences, and some few experiments that have been made, tend to disprove these unqualified vulgar prejudices.

The treatment of an ordinary case of strangles is an affair of peculiar simplicity. The common notion is, that, unless an abscess form and be evacuated, the animal does not derive that constitutional benefit 
which nature intended him: this is one of those vulgar chimeras however that seems not, upon any rational grounds, to be deserving of more attention in practice than is requisite to appease those who are determined to follow the humoral or farriers' pathology. I perhaps may be censured by the profession for what I am going to observe, but I cannot, with justice to my own experience, make a display of what may be really set forth in so few words; viz. that all the treatment a common case of strangles requires is a blister to promote the suppurative action-if I may be allowed the expression, to spur nature onward to the end of the process. Professor PEall has been at considerable pains to show the inutility of poultices and fomentations; but I really have neither space nor inclination to follow him in so unprofitable an exposition. Suffice it to say, that they are troublesome in their application, comparatively of little effect, and quite unnecessary. Steaming the nostrils with scalded bran in a nose-bag is even prejudicial : it aggravates, by its suffocating tendency, that soreness of throat and difficulty of breathing which it is generally intended to relieve. Those who may feel disappointed with this simple view of the case, will find ample satisfaction among the digestive ointments and alterative balls and other farragos recommended by WHrTE. Catarrhal, pneumonic, or other accidental symptoms, must be met by appropriate measures. In regard to openingr the abscess, and the general management of the case, I must refer the student to the account of abscess given heretofore $*$.

* Part I. Lenture ix. "Diseases of the Cellular Membrane." 


\section{LECTURE LXXVII.}

\section{On Glanders and Farcy.}

W ITHOUT taking up time and (what is here of more value to us) space to make any inquiry into the origin and propriety of these vulgar but well-understood appellations for two of our most important diseases, I shall forthwith enter upon this highly interesting subject with an exposition of the doctrine I have carried about with me from my pupillage, for which I am chiefly indebted to the researches of the present Professor of the Veterinary College. It would have come with many advantages from the Professor's pen which I am apprehensive it will now be deprived of; but, situated as I am at present, my duty requires me to put it into the form of a lecture; and I shall use my endeavours to do so, as near as I can, after the manner in which it was orally conveyed to me.

Professor Colmman makes a division of glanders into two forms or species: acute and chronic. That species is acute, which, like other acute diseases, proceeds regularly through its course, and ends in death; that chronic, which, so long as it remains so, will not destroy the animal. This is illustrated 
by what happens in chancre and buboe, and (venereal) gonorrhoea ; the former require the administration of the specific, the latter will in the course of time cease spontaneously.

Acute Glanders may be defined to be, a specific inflammation and ulceration of the Schneiderian membrane, more particularly of that part of it covering the septum : that appearing to possess a higher degree of susceptibility. It is generally attended with tumefaction of the submaxillary lymphatic glands : which tumor is simply the consequence of irritation.

The disease has been long known to be contagious - to be communicable through the medium of contaminated stables, and by inoculation; hence it has been concluded that it had no other origin but contagion. Most physiologists indeed have supposed that cutaneous diseases could not arise from any other cause; and there are certainly some animal poisons whose operation appears to favor this hypothesis; but, in prosecuting our investigations, it would seem that every such poison is governed in its operation by certain laws peculiar to itself : as different medicines produce different effects, so the different poisons of contagious diseases appear to possess peculiar properties, though the diseases themselves all in common admit of propagation by contagion. There are several diseases affecting the human subject, that, according to general opinion, can only be generated by contagion; such are syphylis, cow-pox, small-pox, measles, and hooping-cough. When a person has contracted any one of these diseases, it is said that he has been in the vicinity of contagion or infection; and it may be impossible to prove, beyond all suspicion, the contrary; but, what 
was it that first bred these disorders? Not contagion; but a combination of certain causes; and, being once engendered, they became contagious. : We have examples of this in jail and ship fevers, and in dysentery: these, once generated, become contagious; notwithstanding no such cause could possibly give them origim. Itch, the Professor believes, is often produced in the absence of contagion: it is bred in persomal -uncleanliness. Why, then, may not hooping-cough, small-pox, \&c. arise spontaneously, i. e. from the same - causes which originally produced them? Those who are most conversant in the habits and diseases of horses, snow know, that glanders, although demonstrably constagious, much more frequently larises from other causes: it is a disease that rarely or never spreads anong horses at pasture, though a glandered subject may have been grazing among them; for, we Mearn from experiment, that, although the disease-is communicable by contact, the poisonous matter must be applied to a part bare of hair, and that, even then, the chances are in favor of the animal escaping infection unless the part have previously been; or happen

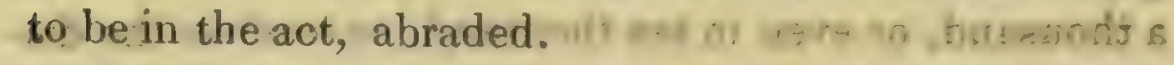

a) There are two casual experimental results by which practitioners, in investigating this subject, haxes suffered their judgment to be misled, and thence have come to erroneous conclusions. The one is, that, because a horse has been subjected without effect to insculation, ergo, the animal from whom the matter of infection was taken, cannot be glandered; the other, that, because the former became glandered; ergo, the latter must of necessity have the disease. There -are-two circumstances absolutely and indispensably 
necessary for the production of a disease by contagion:-the application of the poison, and the susceptibility of the animal or part to which it is applied. You may inoculate without success from insusceptibility of the inoculated subject, on the same principle that you may administer the same doses of aloes to two horses, and effect violent purgation in one, but make no impression upon the other. It is by no means uncommon to see persons inoculated for cowpox and small-pox without effect: the explanation of which is, that they are not susceptible of that degree of poisonous excitation at that particular period. Again, it has been argued that a disease could not be glanders from which a horse recovered, even though it showed every characteristic outward sign of glanders. But the very groundwork of this position is untenable: the Professor has seen (and so have I) several cases of spontaneous cure from chronic glanders; and evidence may be brought forward of recovery even from acute glanders combined with farcy.

So far from contagion being the common cause of glanders, the Professor estimates that not one horse in a thousand, or even in ten thousand, so receives the disease. The poison of glanders is bred and diffused in an atmosphere rendered impure by repeated respiration, and by gaseous impregnations from the dung, urine, and perspiration, emitted in hot and foul stables. No vital being, neither animal nor vegetable, can maintain life in the total absence of pure air; and, according as an animal is from nature habituated to purity, so, generally speaking, it would seem that he suffers from atmospherical contamination. There are several sorts of vegetables that cannot be grown (or at least to perfec-

PART III. 
tion) in the vicinity of London, in consequence, we believe, of the impurities continually floating in the atmosphere; whereas, there are animals (such are rats and mice, and we may add bots) who enjoy health in the most confined and noisome situations. Man can withstand a contaminated or poisonous atmosphere, it would appear from his habits, much better than the horse: we join house to house, form villages, towns, and large cities; and we live thus crowded together with seeming imnocuousness: still, to obtain specimens of wellgrown forms and robust health, we must go into the country and select them from among the husbandmen; for in large and populous towns, instances are always presenting themselves of rickets, scrofula, consumption, \&c. \&c. The horse is an animal destined by nature to breathe an atmosphere of the purest kind; in proof of the salubrity of which to him, suffer him to remain in his native fields, and he will livelong and ail nothing. But, bring him once into a state of domesticity, place him in a confined situation, in which he is compelled to breathe air that has been already respired, not only by himself but perhaps by other horses also, air impregnated with the exhalations from the urine, dung, and perspiration, and you sacrifice him a victim to malignant and fatal maladies. And none of our domestic animals, no more than horses, can tolerate this with impunity. If poultry are kept in a confined place, they breed what is called the pip, which is a very destructive disease among them. Hogs, under similar circumstances, engender the husk, a species of pulmonary phthisis. Even plants, unless they are occasionally supplied with fresh and pure air, will wither away and die in our greenhouses. These morbid consequences arise not from any deficiency in 
the vital or oxygenous part of the air ; for, it is found by experiment, that there is proportionably as much oxygen in the atmosphere of the closest alley in London as in that which encompasses the hills of Highgate: no! these deleterious effects are ascribable solely to the animal poisons contained in the atmosphere, which are not only inhaled with the breath, but probably taken in with the food also; be that as it may, however, through one or both of these channels the poison becomes absorbed into the system, corrupts the whole circulating mass, and breaks out in local forms in various susceptible parts of the body. Therefore it is, that, in the degree in which a stable is foul and heated, from want of ventilation, we find its inhabitants the subjects of glanders, farcy, opthalmia, \&c. we seldom receive these cases from gentlemen's stables, because in general they are well-constructed, and kept clean, and do not contain many horses; but in collieries, breweries, posthouses, coach establishments, \&c. where the stables are filthy from the dung and urine which stagnate in cavities in the pavement, for want of proper sewers to carry them off, and where the men are suffered to add to the mischief by plugging up every air-crevice they can find, we are continually witnessing the ravages of these very formidable diseases. Farmers' stables, though no better or even worse in their construction, do not appear to tum out so many glandered subjects; a fact that admits of reconciliation with what has been advanced, from the circumstance of their stables being in general very capacious, and many of them in too ruinous a state to admit of exclusion of the external air. The Professor was first led to adopt this notion of the spontaneous origin of glanders and farcy from an occurrence

2 ; ? 
in our cavalry service which came to his knowledge. Many years ago (I believe about 1796) there was an extensive encampment upon Dover heights, from which the horses could not be removed until the autumnal season was far advanced, in consequence of the stables intended for their reception not being in a state of readiness : now, the stables were newly erected ones, notwithstanding which, great numbers of these horses, though previously in perfect health, soon after entering them became diseased ; the greater proportion contracted grease, but several were attacked with glanders and farcy. He has since also received peculiarly satisfactory evidence of this in two memorable instances, in which stables which were hot and foul, and had from time to time turned out several glandered horses, were rendered equally salubrious with others adjoining them by proper ventilation and attention to cleanliness.

By this time we shall have received some striking illustrations of what was advanced in the outset, that every animal poison is regulated in its operation by its own peculiar laws: were it not, most wisely, so ordained, the whole animal creation must long before now have been exterminated, If man had been susceptible of contracting disease from horses, oxen, hogs, sheep, dogs, \&c. and these animals, in their turn, could have taken human disorders, all must have lived only to act their dreadful parts in the work of universal devastation.

We now come to the relation of that celebrated experiment of the Professor's, by which, not only the contagious, but the constitutional nature of glanders is proved beyond all doubt and idle speculation-that experiment which goes to disprove the assumption of 


\section{On Glanders and Farey.}

Mr. Hunter, that the blood itself was never diseased. Of a horse affected with acute glanders, the Professor laid bare the carotid artery and jugular vein of the same side, and around each vessel placed a ligature. A pipe, furnished with a stop-cock, was then inserted and fastened into the artery; which was made to communicate, through the medium of a tube (a ureter, I believe) with another pipe introduced into the jugular vein of an ass: this animal having been previously bled until he fell from exhaustion. In this manner, blood was conducted from the artery of the horse into the vein of the ass until the latter showed every sign of perfect resuscitation. A circumstance occurred however in the revival of the ass, which, though it did not affect the issue of the experiment, may serve as a warning to future experimentalists ; and that was, that, in consequence, it was thought, of transfusing more blood than was requisite, the ass appeared to be puffed out or swollen all over the body; this however was relieved in the course of four or five days, in which time the animal became glandered in a most violent degree: in confirmation of this, other asses were inoculated from this one, and they all unexceptionably shared the same fate. The loss of blood sustained by the horse was not sufficient to take away life.

Acute glanders, as was said before, consists in a specific inflammation and ulceration of the membrane lining the nose ; by specific is meant, an inflammation not attended with the usual phenomena. If the inflammation could be as well recognised by any characteristic appearance as the ulceration is, then the horse ought to be pronounced glandered prior to the supervention of the ulcerative stage: to this there are analogous instances in the human subject. No surgeon decides on a case 
of syphylis before chancre makes its appearance, or on small-pox until pustules have formed. We may safely lay claim to two discoveries respecting these diseases. One is, that the whole mass of blood has been found to be contaminated. The other is, that glanders and farcy may be, and are commonly, produced without the agency of contagion. Mr. HuNTER concluded that the blood was never in itself diseased because he could inoculate with it in small-pox and syphylis without infecting the subject; whereas, if he made use of lymph, he produced the disease. This is no proof however that the blood contains no morbific matter ; for the poison mixed with it may be, as we now know it to be, in too diluted a state to take any effect; though, in the purulent discharge, it appears to exist in a concentrated form. On the same principle, a person may drink a tea-cupful out of a pailful of water containing a certain quantity of arsenic with impunity; but, should he take a quart or a gallon of the same fluid, he may probably experience from it deleterious effects. Aloes itself is a poison exhibited in large doses. Another proof of the blood being diseased, is, that syphylitic infection will frequently create disease in the throat: how could the poison get there but through the medium of absorption and circulation? Be this explicable however as it may, we have proved the fact beyond all doubt and dispute by the test of direct experiment.

Glanders is not so prevalent in the summer as in the winter season; and it has, in several instances, been known to be epizootic, particularly when horses brought from camp or other external situations have been returned into warm and unventilated quarters. If precautions were taken to properly ventilate stables, the disease 
might be altogether eradicated. In further proof of the disease originating without contagion, we have instances of glanders breaking out among horses that have been embarked in a perfectly healthy condition on board of ships entirely new. In the expedition to Quiberon, the horses had not been long on board of the transports before it became necessary to shut down the hatchways: the consequences of this were, that the horses were almost suffocated with heat, and that almost all of them disembarked either glandered or farcied. The malady which broke out among the men engaged in the Walchren expedition, attacked almost all of them, hence it was considered to be a contagious disease; afterwards however it proved not to be, nor was this assumption required to explain its endemic character, for they all (if the expression may be allowed) ate it, they all drank it, and they all breathed it. It is for want of reflection upon these points that people are so often differing about the contagious and non-contagious nature of diseases. It has been observed, that glanders is mostly present where grease is prevalent; indeed, this fact led SAYNBEL to say, that grease was a cause of glanders: but, in truth, it is no more a cause than dung and urine are causes; it is simply operating as another source of atmospherical impurity. Under such circumstances, the fetor of grease will predominate, as the stench of a goat will, over the effluvia arising from the excretions and secretions; and it was the observance of this fact probably that gave origin to the vulgar notion of the salutary influence of goats kept in stables.

The acute glanders is the same disease, in regard to the nature of the poison, as farcy and chronic glanders; the Professor however is not so confirmed in his opinion 
concerning the affinity of the last as he is in respect to farcy. Acute glanders hardly ever proves fatal without farcy making its appearance before death; on the other hand, farcy rarely or never of itself puts an end to life, it being superseded commonly by acute, sometimes by chronic glanders. Independently, however, of these practical observations, we have shown their essential identity by direct experiment: we have produced farcy by inoculation with the poison of acute glanders; and acute glanders by inoculation with the matter of farcyl: we do not always succeed, it is true, but one case proves as much as a thousand.

1. Chronic Glanders commonly affects but one side of the head; if, therefore, a discliarge makes its appearance from one nostril alone, that of itself is strong presumptive evidence of the presence of this disease. This partial flux cannot come from the lungs, for, if it did, the other nostril would discharge too; it must have its issue from some part anterior to the larynx, consequently it can have no other source but the membrane of the nose or that portion of it lining the sinuses. Should it come from the nose, the menabrane; most likely, will have a redder aspect upon that side of the septum than upon the opposite, or there may be a disposition to, or actual, ulceration. If the nasal discharge is considerable, and, at the same time, the animal to all appearances is in the enjoyment of good constitutional health, that is a circumstance which should serve to strengthen your suspicions: people cannot conceive how it is a horse can have glanders so long as he eats and drinks and does his work like one in perfect health; this very fact, however, I repeat, is corroborative of an unfavorable prognosis: Another circumstance to be attended to, is, that the nasal flux 
bas little or no fetor: offensiveness of breath is pretty certain evidence that this disease is not present; not but what the two may exist in the same subject, but; fetid breath commonly proceeding from the lungs; and this chronic discharge coming from the sinuses of the head, the one is not in any way necessarily connected with the other. In glanders, the nostrils are contracted and gummed with inspissated discharge; but the flux is not offensive, or, at least, to the same degree as pulmonary fetor is. Again, in glanders; generally speaking, there is on the same side from which the discharge comes, a defined swelling of the submexillary lymphatic glands, which is attached closely and immoveably to the side of the jaw : if it is a tumor of considerable size, one that is diffused and extends inwardly, or one that is very moveable nnder the fingers, most likely it is not lymphatic, and therefore not connected with this disease. So far as the Professor's abservation has gone, no such glandular swelling happens in common inflammation. .... In chronic glanders, then, the general health, appetite, spirits, \&ce. remain unimpaired; there is simply a discharge from one nostril, which is unaccompanied by fetor, and a circumscribed immoveable tumor under the jaw on the same side. In some cases, however, the flux comes from both nostrils; here, commonly; both sets of glands are tumefied, the nature of which swellings will serve to direct the diagnosis; in addition to which, most probably, the animal's health continues good, and the discharge is not offensive. But, if cough is present with such a discharge, the submaxillary tumefaction uniformly diffused between the sides of the jaw, and there are feverish symptoms 
and evident impairment of the general health, the lungs are probably in this case the seat of disease. Still, in order that we may be certain about the existence of chronic glanders, we have no occasion to implicitly rely even upon these signs; for we may at once decide the point by the test of practical investigation. We have only to perforate (with a spill-gimblet) the frontal sinuses, and inject some clean tepid water into them : should the sinuses be healthy interiorly, the fluid will run from the nose either limpid as it was thrown in, or merely be tinged with blood; whereas, in a case of disease there, the water will carry down with it the matter lodged in the cavities. It is not uncommon, in this disease, to obseve a horse discharging profusely for several days and then suddenly cease running altogether: this arises either from inspissation of the matter collected, or from the effusion of adhesive matter, within the sinus, which settles at the bottom of the cavity and plugs up the aperture by which it communicates with the chamber of the nose. During the interval of suspension no sign of disease remains but the submaxillary tumefaction; there is no. discharge, and consequently there can be no source of contagion: but, the collection of matter continually augmenting, at length the plug is forced out, and the flux returns with more virulence than ever. In Smithfield, it used to be a common cheat to sell a horse having this disease for a sound one, the trick consisting in stopping up the nostril of the affected side with a piece of sponge, which, of course, received and imbibed the discharge.

Though we have no specific remedy for chronic glanders, no more than we have for acute, the Professor 
has seen more cases of recovery from this than from the latter disease. When the discharge early in this affection becomes profuse, and continues long so, it will end, every now and then, in a spontaneous cure, as is the case so often with gonorrhoa; at other times, the flux will persist and run for years, and the horse, so long as the disease remains chronic, maintain his full health. Many horses of this description are to be found, in various parts of the country, working in road-waggons, brick-carts, agricultural machines, \&c. \&c. nevertheless, the disease is not only incurable by art, but is contagious; the matter emitted from the nose has the property of propagating by contact either acute glanders or farcy, or even both.

FARCY affords, of all three affections, the most conclusive evidence of the production of the poison in the absence of contagion. It may be defined to be, an inflammation and suppuration of the superficial order of absorbents: it does not affect the deep-seated order - they do not appear to be susceptible of the irritation. This fact is not more extraordinary than many analogous ones to be found in human medicine, such is the case in respect to itch, small-pox, measles, gout, \&c. itch does not disease the stomach or bowels, gout is not a cutaneous malady: certain parts are liable to certain diseases, and the skin of the horse, as well as that of man, has its peculiar affections, among which we may rank mange, grease, various eruptions, and farcy. The extraordinary circumstance connected with the history of farcy, is, that, though it is a contagious disease, it is rarely or never generated by contagion; indeed, the Professor even doubts the fact, though not the possibility, of its ever, under ordinary circumstances, 
taking its rise from contagion. For, in order to com municate farcy, it would be necessary that the matter from the nose should come into actual contact with some bare or abraded part of the skin; and this, when we come to consider how uniformly the skin is covered with hair, and the mode in which we confine and stall our horses in this country, is next to impossible in the stable, and is very far from being likely to happen even at grass. There would be greatly more chance of glanders being communicated in this manner; for, as the horse is an animal that takes cognizance of every thing: by his nose, one subject might besmear the pannels or walls of the stable with the poisonous matter and another sniff it off. With respect to one farcied horse communicating the disease to another, we may be very positive in refusing credence to this; indeed, one might go here so far as absolutely to deny everything but the interference of art. In fine, where there is one case of farey that can be satisfactorily traced to contagion, the Professor reckons there are a thousand in which its origin is spontaneous, or in which it is a concomitant. of glanders. In inoculating for farcy, simple abrasion is all that is necessary; indeed, should the puncture with the lancet be made so deep as to penetrate through the skin, the probability is that glanders, instead of farcy, will be produced.

No part of the body can be said to be exempt from farcy, though it shows a predilection for those places where the skin is thinnest, and appears in situations remote from the heart: its common seat is the inside of the thigh; but we very often find it upon the inner parts of the arms and upon the breast, also along the neck, arid upon the cheeks and lips, in which last case greater 
apprehensions are always to be entertained of the supervention of glanders. Occasionally, it commences its attack upon the body. The ordinary appearances or signs of farcy are-corded swellings in the skin, following the course of the superficial veins, and traceable into lymphatic glands, either in the groin or breast, or under the jaw, which, as well as the tumefied absorbents, are hotter than the surrounding skin, and tender on compression. After a time, these cords assume a knotted appearance; regular chains of knots or lumps are formed in the skin showing most distinctly the course taken by the superficial absorbents: these knots are what farriers have denominated farcy-buds. When first formed, they consist of effusions of adhesive matter but, as the disease advances, they gradually take on a suppurative action, the progress of which change is denoted in them by their growing softer, hotter, and greatly more tender to the touch. At length, abscesses are produced : these burst, and the limb or part affected becomes lined with a chain of small, circular, foul ulcerations. The characteristic knotted tumefaction of the absorbents is attributable to the intercepting valves, parts which do not seem to be susceptible of farcied irritation; for, if one of these diseased vessels be examined, perfectly sound partitions of membrane will be found between the knots, which cannot be any thing else but the valves. It often happens, however, that the buds, instead of undergoing an inflammatory process; degenerate into hard lumps, having but little heat or sensitiveness, which ultimately become converted into schirrous substances; in which state you may cut or burn them without the animal evincing much pain or uneasiness, or perhaps seeming consciousness at 
all of what is doing: so void are they of sensation when they have undergone this change.

There is a form of this disease which the farriers call watery farcy, as if it was a distinct species; whereas, the pathology of such cases appears to be, that so many of the absorbents being unable from disease to perform their functions, the result is œdema in combination with farcy : these cases manifest a more fearful disposition than others to run into glanders.

Farcy may be said to have various terminations. The most common of all sequels is that of glanders. Cases ending in apparent health however are by no means uncommon; nay, cures are every day said to be performed; and, no doubt, there are many cases in which perfect health is restored; but the majority, the Professor apprehends, are only instances of temporary arrest or suspension of the disease; for, in too many of them, months, and even years, afterward, swellings are discovered under the jaw, and the subjects ultimately become the victims of acute glanders. The fact of our seemingly being able to cure one disease, and certainly not the other, would appear to operate against the affinity of farcy and glanders: (venereal) gonorrhœa and chancre however furnish us with analogous cases in human medicine; one will cure itself, the other can only be eradicated by the exhibition of the specific. At other times, these cases will end in geneval thickening and induration of the affected parts; the skin covering the enlarged absorbents will become morbidly thick, and grow callous to all ordinary stimuli; and, in this condition, the animal may enjoy, to every appearance, good health for months, or even years: but, let it be remembered, that the seeds of glanders 
are still probably luxking in the system, and only awaiting the stimulus of re-action to break out again in some visible and more destructive form, and carry the animal to his destined fate; as if it were to show us the radical inefticacy of our remedies, and the instability of any remission, however long, we may obtain on such dubious grounds. You may have acute and chronic glanders and farcy in the same individual; though it never happens, I believe, that acute glanders or farcy ends in chronic glanders.

In incipient farcy, it is proper to inflame the skin covering the affected absorbents by rubbing the blistering infusion upon it, or by the employment of the actual cautery-firing in the course of the corded swellings : these escharotic remedies destroy the cutaneous surface, and, so far as regards the local affection, are likely to excite healthful and restorative action. Unless there be pus effused; and then the system is most probably infected, (by absorption of the matter) even should the disease bave been purely local in the first instance. Here then, certainly, (and probably at all times and in all cases) constitutional remedies should be resorted to. That which in the Professor's opinion holds out the most favorable prospect, is copper, either in the form of the cupri sulphas or cupri acetas, which may be combined with some terebinthinate: in its administration, it is advisable to commence at once with large doses*, and repeat them every one or two days, according as
* R Cupri Sulphat. 3vj.-3viij
Terebinth. Vulg. $\overline{3} 1\}$.
Farinæ q. s. ut. f. Bol.

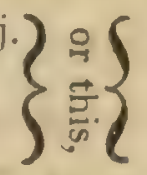
Bx Cupri Acetat. $3 j$.
Terebinth. Vulg. 313 .
Farinæ q. s. ut. f. Bol.

or, the copper may be given in a state of solution, should the ball occasion nausea and loss of appetite. 
the stomach will bear the action of the metal. Exercise, good air, full diet, and turning the subject loose into a roony box, are serviceable auxiliaries. - If abscesses have already formed, apply the heated budding iron to them singly, so as to burst them and destroy the morbid secreting surface; and, at the same time, cover the parts with a liquid blister: by such measures, in combination with the internal exhibition of either blue or green vitriol, the Professor has seen the disease, in many cases, to all appearances, subdued : any prognosis however drawn from such results should always be properly guarded. When the sloughs have separated. and open ulcers are exposed, they may be dressed daily with a solution of copper, or alum, or zinc; or the castery may be re-applied; according as the case seems to require.

With regard to remedies for glanders, either in its acute or chronic form, the Professor has nothing to offer. He has prescribed, by way of experiment, almost or quite all the mineral and known vegetable poisons: preparations of arsenic, antimony, copper, zinc, mercury, \&c. hellebore, aconitum, digitalis, hyoscyamus, cicuta, belladonna, \&c. \&c. have been, in various condemned subjects, internally administered; but all without any specific or curative effect on this awful and obstinate malady. 

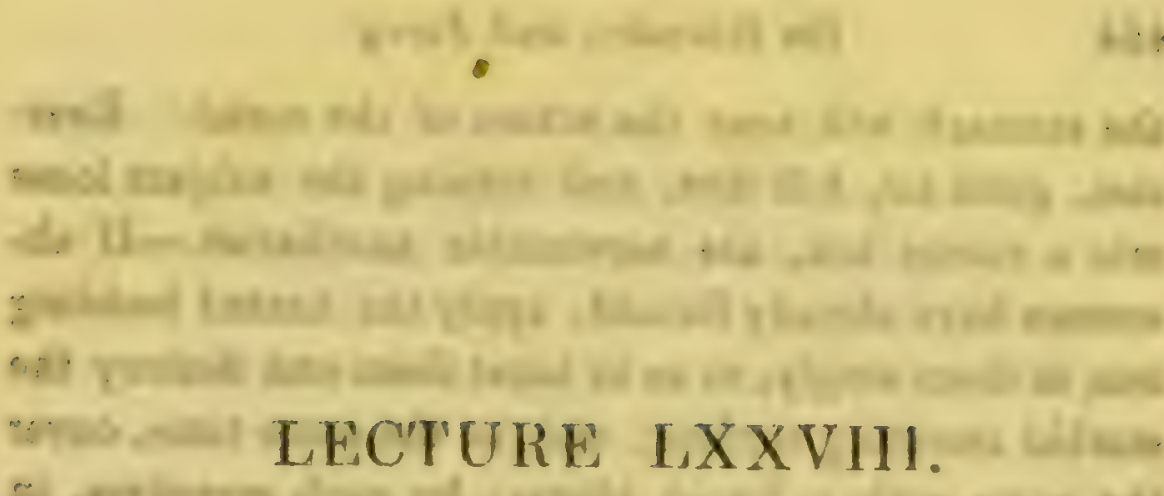

Glanders and Farcy continued.

I. $\mathrm{N}$ the past lecture I have endeavoured to convey a knowledge of the doctrine propounded on these subjeets by Professor Coleman ; I shall in this, my concluding lecture, submit an exposition of that promulgated on the same topics by Professor DUPUY, whose celebrated work*, which was published in 1817, none to our eredit on judgment, remains to this day virtually cunknown in British veterinary medicine. This pro ceeding will render the doctrines of the highest British and Continental authorities subservient to reciprocal relucidation, and at the same time will afford an opportunity of drawing some comparison between their mopinions: The facts and observations presented to us by the French Professor are for the most part of a novel

* "De l'Affection Tuberculeuse, vulgairement appelée morve, pulmonic, gourme, farcin, fausse gourme, pommelière, phithisic du singe, du chut, du chien, et des oiseaux domestiques; comparéc à l'affection hydatideuse on pourriture du mouton, du lapin, du lièure, et à la ladrerie "du cuchon. Par M. Dupur, Médecin-Yétérinaire. et Professor à l'Ecole Royale d'Economie Rurale et V'cterinaire d'Al. fort, Membre de la Soriété Médicale d'Fmulation. A Parrs, 1817." 
and most interesting description, and many of the conclusions deduced from them such as are likely to prove of considerable importance to us in our future practice.

The end proposed by M. DuPry in this research was to obtain correct pathological views from a multitude of observations, both on the living and dead subjects, extended to domestic animals in general; and with this view he commences his work with a rapid exposition of the different opinions of the most popular French authors on the subject, not comprising those however who are still living, and brings his historical account to the following conclusion. "It is evident that the ancient authors have not directed their researches to determine the nature of glanders: they observed the outward symptoms, the obvious signs of disease, and they framed hypotheses, and theorized upon conjectures; not one of them has viewed it as an organic lesion, nor suspected it to be so common as it actually is. The moderns have nothing enriched their ideas; they have pursued the same tract: like those who have preceeded them they have not sought for the source of the evil; their writings are really nothing but commentaries; they have developed, amplified, presented us in other forms with what was written before.

"I shall make a division of my labors on the glanders of horses, generally considered," says M. DUPUY, "into two parts. In the first, I shall view the tubercle as an organized production, formed or developed at an indeterminate period; after which it degenerates, deranging the functions of the organ itself, as also those of the economy in general. In the second, I shall show the changes the tubercle undergoes; and relate the phenomena preceding, accompanying, and following its 
degeneration and subsequent ulceration: towards the end of this second period it is that we observe the most striking derangements in the animal economy. These derangements make more or less impression, influenced by a multitude of circumstances connected with the animal and its surrounding agents. In another place, I shall develop the predisposing and determining causes. My principal object being to point out the progress of the tuberculous affection constituting glanders.

\section{"FIRST DIVISION.}

\section{"T'he T'ubercle forms and multiplies.}

"ТнЕ organized production we have named tubercle is the product of a cause which to me appears unknown. Nevertheless, it is under this unknown influence that its component elements coalesce; and to the same cause must be ascribed the different material changes it undergoes.

"The primitive tubercle is a little, firm, grey, hard body, composed of a fine cellular tissue, in which is deposited a substance that proves to be phosphate and carbonate of lime: it is mostly inclosed in a small cyst, appearing to be a production of the tissue in which it is formed.-These incipient tubercles exist in small numbers, and interfere but little with the functions of the affected part. - At this time, the animal enjoys a flourishing state of health, and glander's is unknown to exist. - We next discover, without being able to assign any satisfactory rationale for it, that the tubercles grow more numerous: in which case they occasion a discharge or flux, most commonly from one nostril: this is regarded as catarrh or chronic defluxion, or, should the horse be young, as strangles." The space of time 
during which the tubercles preserve this organization, grow, and increase, (five or six years perhaps) constitutes the first stage: during which they resemble so many poppy seeds.

In the second stage, the tubercle grows soft-disorganized, and degenerates and becomes converted into an ulcer: this ulcerative degeneration is what has been seized upon by LAFOSSE, CHABERT, and other authors, and made the basis of their divisions and distinctions of glanders.- " The ulcerations observed upon the mucous membrane of the nose in the last period of glanders, consequent upon the degeneration of the tubercles, are insulated or united, grouped, or so near one another that in meeting they disorganize a broad patch of the Schneiderian membrane. These alterations depend upon the variety of the tuberculous affection.

"The variety I shall name miliury is the one oftenest met with. The degeneration of this variety of tubercles converts them into little ulcerations, with thin edges, unevenly excavated, resembling pin-holes; with this difference however, that the hole made by a pin would be deep and pointed, whereas these ulcerations are shallow and have indented edges. They are commonly regarded as erosions, often mistaken for the dilated orifices of mucous follicles; but, if they be examined after the mucus in which they are sheathed has been removed and the membrane has been cleansed with water, they will be found to be so many little ulcers. The membrane clothing the septum is frequently covered with these exulcerations, and its surface in places elevated. But they are superficial, penetrating merely through some thin layers of the cellular tissue of the 
membrane, thereby rendering its surface irregular, uneven, and scabrous. Cateris paribus, they exist in greatest numbers over the large veins pervading the septum. They are also found grouped within the doubling forming the internal ala of the nostril, particularly on the left side, following the course of the bloodvessels, and upon the turbinated prominences and their appendices.

"These tubercles, by interunion and simultaneous ulceration, may produce a broad ulcerous patch, threatening destruction to the parts beneath. Though for a time the perichondrium preserves the cartilage, at length it likewise degenerates, even should such a change not be accelerated by the existence of tubercles within its own proper tissue, of which I have received numberless casual demonstrations. - Similar destruction pervades the turbinated, frontal, and maxillary bones. They grow puffy, sivell in volume, and turn spongy; the surfaces of them forming the sinuses become covered (internally) with spongy, unsubstantial, very porous excrescenses; and the bone is deprived of its earthy composition : such are the different lesions met with in horses who die or are destroyed from glanders.

"In this variety of the ulcerative decline, the membrane bordering, even very close, upon the ulcerated places preserves its organization. Prior attacks of inflammation, either of the acute or chronic type, may also leave traces upon the membrane, which then becomes the seat of two perfectly distinct lesions; of ulcerations resulting from the tuberculous affection, and of a fibrous induration, radiated in the form of stars, which is really the result of the consecutive chronic inflammation that has attacked the membrane at differ- 
ent times, and converted it into a fibrous, white, firm, lardaceous tissue, bearing great resemblance to schirrus.

"It follows, then, from what has been said,

"1st. That glanders is really a tuberculous affection.

"2ndly. That it bears the closest analogy to phthisis pulmonalis in the human subject.

" $3 d l y$. That the miliary variety is the most conmon.

"4thly. That the left is the nostril ordinarily affected.

"5thly. That the membrane lining the sinuses is rarely found tuberculated or ulcerated.

"6thly. That a glandered horse may contract a malady of a gangrenous nature.

"7thly. That this gangrenous affection, which is contagious and idiopathic, should be carefully distinguished from the true glanders.

"I hope to set this last proposition out of the reach of doubt by some special cases I have to report of acute glanders, which I take to be an angina gangrenosa or analogous affection.

"I have also observed tubercles in other tissues besides the nasal membrane, principally within the parenchyma of the lungs, within the lymphatic glands, cellular membrane, skin, testicles, lining membrane of the alimentary canal, \&c.

"The lymphatic glands commonly diseased are the sublingual (submaxillary) and the guttural, and the tubercle is mostly the miliary. They first change color, tumefy, and grow firm, and albugineous in appearance, displaying, interiorly, dense, yellowish, encysted miliary tubercles. I cannot determine how long the tuber- 
cles continue in this first stage; though I have ascertained, that, once developed, they become insusceptible of resolution.

"Should glanders be complicated with a tuberculous affection of the lungs, the animal coughs frequently; tires early; perspires freely; later, he loses his vigor and energy; grows washy, soft, and lazy; becomes subject to catarrh, weak eyes, cutaneous eruptions, farcy, œclema, cracks, \&c. At length the digestive functions manifest disorder; the animal has a remarkable bloated aspect; the cellular tissue is blown up, as it were, and pits; the conjunctiva is infiltrated ; the caruncula lachrymalis discolored; the eye has the expression of that of a sheep suffering from rot; the gums are pallid; and the under eyelid of the affected side juts into a prominent circle or duplication. If put to hard work, such horses rapidly decline in condition; and yet, should they be destroyed, fat is found in abundance upon the belly, and about the heart. It is at this period that the glanders becomes complicated with farcy.

"I cannot regard farcy buds in any other light but as scrofulous tubercles. They become developed under the same influences, possess the same organization, undergo similar conversions, and decline in the same manner as pulmonary tubercles. As for their frequent degeneration into cancer or carcinoma, cannot we attribute that change to caustics or the actual cautery, which are frequently applied to the buttons of farcy in a manner at once inconsiderate and abusive in the extreme ?I shall conclude these considerations by observing, that the glanders or phthisis of the nasal membrane will turn, under some circumstances, into an affection of a 
cancerous nature; while, under other circumstances, it becomes complicated with a disease analogous to the typhus of horned cattle, and the confluent scab (clareau comfluent) of sheep, which proves very fatal in combination with typhus.

"Farcy is often a local and an original affection. Nevertheless, will any one pretend to draw an induction from these facts to prove that it is a different disease from glanders, and that it cannot originate from the same cause, although farcy admits of being cured, while glanders has resisted every means hitherto opposed to it? This is not the first time that we have heard of one variety of the same disease being curable, and another not. Schirrus disappears without relapse under many circumstances. And yet it is not so with cancer, carcinoma, \&c. These observations serve to reconcile the contradictory accounts of veterinarians, when we find one declaring that farcy is easily cured, and another maintaining a directly opposite opinion. The probability is, that they have been treating different varieties of the disease : in one case the farcy may have been local ; in the other, constitutional."

M. DUPUY next observes, that the process of degeneration in the absorbent glands seems to proceed from the circumferent parts to the centre; the fact however is hardly confirmed; if it were, as he adds, "it would form a remarkable particularity. One important consideration however is, that these tubercles multiply under the influence of cold and wet, at least I have found that horses so exposed had a greater number of tubercles in their lymphatic glands and other parts of their economy." These (glandular) tubercles grow soft and degenerate, become abscesses, and convert the 
whole interior of the gland into little cysts, whose parietes are dense, cartilaginous and occasionally bony. The cysts in inveterate cases are found in a state of ulceration, similar to that which destroys the membrane of the nose.

So far from being favorable, the Professor says, " suppuration announces the advanced state of the dissease, and tends, not to heal, but on the contrary to destroy the morbid tissues. It is a bad practice therefore to promote suppuration. In fact, at this period, the frontal and maxillary bones form a very perceptible prominence, most commonly on the left side, which latter circumstance adds to the ominousness of the prognostic.

"I have been pointing out all that relates to the different varieties of tubercles. Furthermore, the proper tissue of the lungs is altered by, their presence; the parts surrounding the tubercles change their condition; they become red, dense, hepatized, and more friable: the same as it does after fatal acute peripneumony. In other horses, the contiguous tissue becomes firm, condensed, whitish, and resembles fibrous substance. The indurated part is with difficulty divisible with the bistoury, and seems to me to be the effect of chronic inflammation. I have observed a third alteration, especially after the ulcerative decline of the tubercles; in which case the parenchyma is softened, blackish, as if it were decomposed, exhaling an insupportably fetid odor, which I cannot compare to any thing else but cariosity.

"It is now time to return to the description of the pulmonary tubercles. - I have observed three varieties of tubercles in the lungs: the miliary, the pisiform, and the unciform. These morbid formations are com- 
posed of an envelope or cyst, and of a whitish substance, easily crushed between the fingers, which Messrs. Dilong and Lajillardiere have found to resemble osseous matter. Very considerable depositions of this bony substance are occasionally seen in the proper pulmonary tissue; especially in the ox species. -When these tubercles are large, their number is limited; but the miliary kind are innumerable. While forming, they are firm, organized, and always found in the course of the blood-vessels, whose calibre is singularly increased; they grow and become developed like any other organized bodies, without our being able to offer any rationale of the process, or of the space of time they continue organic, prior to their mollification and degeneration : they commonly end in ulceration and destruction of the pulmonary substance. The lungs of such animals present vomicæ or cysts of various sizes, contain a thick reddish matter, at other times a more liquid cheese-like matter."

M. Dupuy has also found small, white, hard, miliary tubercles within the parenchyma of the liver, often in great numbers; encysted pisiform tubercles in some cases within the parenchyma of the kidney; and, much oftener than either within the liver or kidney, encysted tubercles within the testicles, constituting in them a kind of sarcocele, which is not always eradicated by castration. "The epididymis also participates in this affection.- Sarcoccle occasioned by external violence admits of being cured by castration; but if it originates in the tuberculous affection, the operation proves inefficacious.

"As I said before, if farcy is regarded as a sequel of scrofulous disease, the cellular membrane and the cutis vera, which are its seat, may he said to frequently un- 
dergo similar changes; indeed, perhaps grease (e'tux an $x$ jombes) results from the same caluse. -1 may add that the same scrofulous action is also marked in the bones, periosteum, and cartilages, \&c. There is a skeleton, in the Veterinary Museum at Alfort, the bones of which are crooked, especially the radii and tibir: these bones, together with the under jaw, are covered with many, irregular, spongy exostoses. There is in the same cabinet, a scapula perforated from the effects of a farcy tumor; also enlarged puffy cannon bones, sequels of the same disease.-Specimens of nasal septa softened and destroyed by miliary tubercles." - In fine, "it is manifest that this scrofulous or tuberculous evil spares no part of the system.-Animals who are the subjects of it mostly die about the sixth or seventh year of their age: an additional consideration that ought to engage us in researches in. what relates to this fatal malady, in order that we may be able to decide, by positive experience, whether or not it be hereditary; for, then, we shall have hit upon le véritable presérvatif.

" In reporting particular authenticated cases, it appears to me to be of great importance to prove, that glanders, at the time it first invades internal parts, is latent; from which it may be inferred that the elements composing what I have named scrofulous tubercles, coalesce under the influence of causes which appear to me to be hereditary, as also under that of cold and humidity. Though the cause were unknown to us, however, the effects I am describing might equally the same exist. The tubercle forms without our being able, by any certain signs, to direct attention to the time of its development. In fact, the animal, the subject of these active morbid phenomena, cnjoys good health up 
to the disorganization of the tubercle, and those internal changes of it which end in its mollification and ulceration.

"How to determine what constitutions are most liable to become glandered? How to obtain a knowledge of the circumstances concerned in working this tuberculous conversion in organized parts? under what influences it takes its origin, proceeds, and terminates? how far it is hereditary? how cold and humidity can operate in disposing the animal economy to breed this malady? to what extent the impressions of cold and humidity require to be made to excite the development of tubercles, the absorption of the earth of bones, or the production of hydatids? These are questions I cannot answer."-Though observations would lead us to reduce these changes to two principal causes: "namely, the influences of external agents and the crossing of breeds." Change of climate is certainly one general cause of constitutional derangements, especially when the emigration takes from the North to the South.

Horses disposed to glanders are characterized " by their ill-conditioned, lank appearance; by their being soft and early fatigued; by the irregular and difficult protrusion of their teeth*; by the coarseness of their heads, and their heavy inexpressive looks. The specific opthalmia (la fluxion lunatique) affects the horses of certain northern countries; their growth is not kind; these horses are called washy; (chevaux decousus;) the carcass is let down, the limbs become long and lank.op. cit.

* "La protrusion des dents est irregulière, difficile." pag. 58. 
I shall conclude this part of our subject by observing, that the different names which have been given to incipient glanders have contributed to banish truth from our inquiries. Affections, which are but consecutive, have been described as essential. Authors in general have regarded strangles as a distinct disease, but GilberT, who has taken a more enlightened view of the subject, considers it as an effect of the same specific cause as glanders. The symptoms, he observes, of strangles do not differ from those of glanders. And, if the differences between them be not essential, sufficiently so to constitute two distinct maladies, he cannot but view glanders as an imperfect strangles. Bastard-strangles appears to him to verify this proposition. The malady comes on with all or nearly all the characters of genuine strangles, and yet it is milder in this form. How often, asks this author, does not one witness the disappearance almost all at once of the fever and inflammatory symptoms? The enlarged glands, whose heat and elasticity seemed to announce a speedy suppuration, become, suddenly almost, cold and indolent; sometimes they vanish very rapidly; the flux continues and resists all repellent means; in a word, the horse becomes the subject of the most decided glanders. This, the ordinary sequel of bastard-strangles, is sometimes the termination of true strangles. If, with these facts, notorious to every practitioner, we consider that glanders is acute, inflammatory, and has really all the characters of strangles; that in asses and mules, glanders almost always takes this course, it seems to GrLBERT that it is not possible to overlook the perfect identity existing between two diseases which to this day have been viewed as essentially different. 
"I coincide in opinion with GringR in one point; and that is, I believe strangles, bastard-strangles, farcy, grease, and specific opthalmia, to be very frequently the effects of the same specific cause; and these effects to be ascribable to the tuberculous affection. This is not a speculative or gratuitous opinion; I have reported observations placing it beyond doubt or dispute.

"The tuberculous affection varies in its symptoms according to the part or tissue in which it is seated. I have observed-1st. That it attacks the mucous membrane lining the frontal sinuses, the maxillary, sphenoidal, \&c.

" $2 d l y$. That the mucous membrane of the chambers of the nose is frequently its seat.

" $3 d l y$. That the lungs are often affected.

"4thly. That the sublingual lymphatic glands are constantly altered, disorganized by this disease.

"5thly. That other systems in the economy are equally subject to it: I may mention the skin, the subcutaneous cellular tissue, the testicles and the epididymes.

"One would expect that a disease which attacks parts so unlike in their organization would assume very different forms: this is observed in the present instance.

"I have no need to show that the disease will be more serious and more quickly mortal in proportion to the number of tissues affected in the same animal: proofs of this are not wanting, if such averments required demonstration.

"The progress of glanders in the mucous membrane lining the sinuses is very tardy; which I attribute to the 


\section{On Glanders and Farey.}

liminished vascularity and consequent interior ornanization of that part, to the membrime extended over the chambers of the nose.

"Glanders is observed to be more active when seated in the portion of the nasal membrane investing the turbinated bones or the septum.

"The tuberculous affection advances very slowly when its seat is the parenchyma of the lungs.-In this instance, it assumes the appearance of catarrh, peripneumony, pleurisy even, mourning of the chine, gangrenous peripneumony, \&c. all consecutive effects, in certain cases, of the present affection.

"It is difficult to point out clearly the signs which denote this disease in the sinuses; they are so obscure, that the observation and dissection of a great number of subjects are required to make this variety recognisable. While the animal is living, a slight prominence of the affected sinus may be remarked; the eye is diminished and humoury; (chassieux;) the lids are tumefied ; the inferior one has a prominent doubling, (bourrelet) in it; the conjunctiva is pale and infiltrated, and so are the membrana nictitans and caruncula lachrymalis; the tears flow over the face; the skin covering the affected sinus is thickened, puffed, and likewise the cellular membrane and periosteum. In tapping with the finger upon the region of the diseased sinus, the animal flinches from the impressions, thereby evincing pain there; should he submit to this test, he shuts his eye, with a blinking movement of the lids, when the blow is received upon the diseased cavity; the sound occasioned by the experiment is morbidly dull and obscure, which is an indication that the membrane is thickened, or that the sinus is full of puriform matter. After death, we find that the membrane has undergone 
rather the cancerous than the scrophulous degeneration.

"When the mucous nembrane of the chambers of the nose is affected, the sublingual (submaxillary) glands becone tumefied, and undergo some very variable changes: in fact, they grow, in the same animal. swollen, firm, painful and moveable. Shortly afterwards, they become insensible, diminished in volume, and appear to resume their natural condition; then again, all on a sudden, they begin to swell once more, and in the course of a few days grow larger than ever. "In order to become well acquainted with all the characters which may serve to distinguish incipient glanders from catarrh and peripneumony, whose forms it assumes, it will, I believe, be necessary to revert to the constitution of horses most subject to glanders, and afterwards point out the most remarkable changes the disease effects in the constitution or organization of subjects laboring under it.

"If, after an attentive examination, we find that the diseased horse has been reared in a low, wet, marshy country; if he shows the characters of such an origin, viz. flat feet, long-hairy legs, exuberant chesnuts*, \&c ; or if he has a narrow chest, razor back; if he is high upon his legs, loose-made, \&c.; if he coughs easily;

* "Chataignes." So the modern French writers designate the oviform, horny, hairless eminences, found in the horse species upon the inside of the arms and the hind legs, just below the hocks. For my own part, I am quite at a loss for an English appellation for them, and so I have followed the French comparison, and named them chesmuts. GarSaUlt, speaking of them, says, "Quelques-uns appellent cette corne ergot; mais il vaut mieux, comme plusieurs auteurs, l'appeler chataigne ou lichen, pour la distinguer des ergots, autres parties que nous verrons ci-après." Le Nourveau Parfait Marechal. pag. 5. 
if he becomes soon fatigured, Xc.; if he has been some time exposed to cold and humiclity, and fed through necessity on over-dried, musty, rushy provender, and on that in sparing quantities; if one perceives marks of suppressed œdema, \&c.; if, combined with all these signs, the mucous membrane of the nose is thickened, infiltrated, discolored, as likewise the conjunctiva and membrana nictitans; if one of the eyes appears sunk, gummy, Sc.; if the external nostril is curled in and fouled with the mucus sticking about it, notwithstanding neither tubercles nor ulcerations (vulgarly ealled chancres) are apparent; almost to a certainty an animal uniting these symptoms is affected with glanders in the first stage. If the subject thus characterized is a gelding, and of the northern race; and has inhabited a stable, cold, humid, low, by the side of some ditch or river, \&c. any remaining doubts become dispersed. If the nasal flux is found to be the sequel of catarrh, strangles, or cynanche, and the animal, after recovering his spirits on the cessation of the inflammation, eats and drinks and works as if he were in health, notwithstanding the discharge continues undininished; if the skin is dry and adherent, we may boldly and without apprehension of being deceived, regard the horse as glandered, especially if any ulceration is perceivable towards the ethmoidal sinuses.

"We must not take the disappearance of the outward symptoms of these maladies for radical cures; a case of frequent occurrence, but one in which the tuberculous disposition still remains. In fact, I have seen several times glandered horses considered as cured, and symptoms characteristic of the disease have appeared again after an interval of a month or two. One

PART 111 . 
case is here reported which shows that all apparent symptoms may be suspended for nearly a twelvemonth. This happens when the tuberculous affection attacks some internal viscera, such as the lungs. This suspension or intermission also takes place when the tubercles undergo conversion: so that we find the internal disease increasing, while the external affection is vanishing: away. We must not therefore look upon these changes as certain indications of cure.

"I have related cases of horses destroyed in a few days by acute diseases, which on dissection have presented tubercles in the nasal membrane or pulmonary parenchyma. These lesions evidenced the existence of the tuberculous affection; which proves that, with every appearance of excellent health, these horses were attacked with a disease which would have destroyed them at some subsequent period, if the acute affection had not carried them off prematurely.

"From the foregoing observations and facts appear deducible:

"1st. That it is difficult to detect glanders in its primitive form.

" $2 n d l y$. That it remains long latent within the substance of the affected tissues without disturbing their functions.

" $3 d l y$. That in the course of this long period, one of two or three years and sometimes longer, it is confounded with many other diseases supposed to be idiopathic; whereas they are but symptomatic.

" 4thly. That the distinctions between them are not simply affairs of curiosity, but even serve to elucidate the diagnosis.

"5thly. That it is at all times easy to recognise the 
disease on dissection, since tubereles atre found in several of the tissues.

"6thly. That incipient glanders in living animals is not so easily detected.

"7thly. That the perusal of the works of those authors who have written on the subject, convinces us that they have overlooked the first stages of glanders.

" 8thly. That strangles, or rather bastard-strangles, is often an effect of this disease.

"9lhly. That glanders take's on many different forms before it assumes that by which it is characterized.

"10thly. That the incipient or first stuge of glander's of authors, is already of long standing.

"1lthly. That at this period, apparently its onset, it is almost always incurable.

"12thly. That it is of great importance to treat it while the tuberculous diathesis remains.

"13thly. That, once developed, tubercles are no longer susceptible of a favorable resolution.

"14thly. That if glanders can remain latent in horses who enjoy vigorous health, experiments made to determine its contagiousness, may prove fallacious and lead to erroncous conclusions.

"15thly. That latent glanders may, at the very moment the experiment is made, when the poison has been applied to the nasal membrane, break out in the most unambiguous form: thus it will make its appearance during the experiment; but it existed heretofore.

. "16thly. That I have received proof that such experiments are not so practicable as some would have us believe.

"17thly. That the contagiounness of glanders yet remains an unsolved problem. 
" 18 thly. That almost all veterinarians have adopted the idea of contagion; though some have advanced quite the opposite opinion.

"19thly. That veterinarians regard this as a part of the established doctrine; though there are many proofs to the contrary.

"20thly. That I know of no well-conducted experiment in favor of contagion, whereas there are some against it.

" 21 st. That if I raise doubts about the contagiousness of glanders, it is with a view of inducing Government to order some experiments to be directed to this object.

"It is manifest, then, that all has not yet been said on the subject of glanders; and that my work is not come too late.-I shall even leave many points yet to be cleared up.

"Furthermore, observation has shown that the puriform matter coming from the bronchiæ, which is discharged by the nose, does not cause glanders in passing over the nasal membrane, as veterinarians have imagined. Glanders, in my opinion, is a specific disease ; and not a termination of strangles, bastard-strangles, cynanche maligna, farcy, watery farcy, catarrh, \&xc. When the lungs are affected, it is a sequel of the tuberculous disposition, and not a termination of peripneumony. On the contrary, acute or chronic peripneumonies are very often consequences of the tuberculous affection."

\section{Latent Glanders.-Morve Cachée.}

UNDER this head M. DUPUY presents us with twelve cases in detail, which go chiefly to show-The latent nature of the disease, which is such that it is not dis- 
coverable during life by the most experienced practitioner-That tubercles not only exist in the first, but even in the second degree of development in the internal viscera, without deranging their functions, particularly in the lungs-And that the affection disappears for a time, and afterwards breaks out again without any determinate causes.

\section{"Conclusions from the First Part.}

"THE symptoms announcing the development of the tuberculous affection, are very slight and difficult of detection. Taken singly, it would be impossible to make any satisfactory induction; but in the aggregate, they may furnish an able practitioner with some useful eclaircissements. - If, then, the nasal membrane is pale, thickened, and infiltrated; if the submaxillary glands are tumefied and collected in bundles; if the coat is discolored and arid, the skin tight, and there is no perspiration; if the gums are infiltrated and pallid; if, with these slight characteristics, we find that the animal has sojourned in a marshy, shady country; if he has been at bivouac, exposed to cold and humidity; if his provender has been of bad quality, rushy, musty, \&c. ; if he has had frequent catarrhs; if he has had relapses of strangles; if he has had bastard-strangles, chronic or malignant nasal discharges; if he perspires from little work ; if he coughs habitually; if he shows signs of grease or farcy; if his constitution marks him to be of northern origin, to have been reared in a shady pasturage, where he has undergone a sort of over-growth; (étiolement;) if the chest is narrow, the withers razored, the belly sunken, the feet flat, the chesnuts prominent, the coat and long hairs strong and abundant; if he has heen castrated; if he 
las a slight flux from the left nostril alone; it is very probable that in such a horse tubercles are developed, notwithstanding even that the eye cannot discern any in the nose. It becomes our duty therefore to place him in such a state, and submit him to such a regimen, as will be hereafter described, when I come to treat of the curative, and, above all, of the preservative means.

\section{"SECOND DIVISION.}

"The Tubercles grow soft, and, in becoming disorganized, ulcerate.

"IT requires likewise some share of penetration to discover the commencement of the period now under consideration: the tubercles being in small numbers, and still preserving their organization, they occasion but very little disturbance to the functions of the organs in which they are seated. It is towards the end of this second period, when the tubercles become disorganized, that the phenomena are rendered manifest, and the functions seriously disordered; the animal still keeps his embonpoint, during the whole of this period, though the mollification the tubercles undergo interiorly disposes him to inflammatory disease: his embonpoint is doubtlessly the reason why these inflammations or phlegmasiæ are attributed to quite another cause and treated as such; little are they thought to be the effects of the tuberculous affection; it will therefore prove very useful to determine well the nature, of these diseases; since at this period we might yet succeed in suspending the progress of the tuberculous affection, though it were not possible to eradicate it. When we come to examine horses who have died of such acute diseases, we find tubercles softened into suppuration. 
if I may view their disorganization or destruction as a veritable suppuration: an expression I introduce here in accordance with general usage.-The tuberculous affection is not then a termination of acute or chronic phlegmasiæe, of nasal fluxes or bastard-strangles, as veterinarians believe, who say, when the inflammatory symptoms are dissipated, that the case is doubtful, suspicious of glanders.-By reading over the cases I have collected, we may satisfy ourselves that the tuberculous affection displays different lesions which may lay the foundation for several varieties. I believe that, in the mean time, I might insist that it often degenerates into a schirrous or cancerous affection. This transformation is frequently manifested in the disease we call farcy.In summing up the cases I have reported, it will be remarked, that of those horses who had only the nasal membrane affected, there is but one case in which the right nostril was the seat of disease; whilst there are eight cases having the disease on the left side. It is without doubt a particularity that the membrane of the left chamber of the nose should be most frequently the seat of the tuberculous affection; it is not very favorable to the idea of glandered contagion; it even contradicts what is advanced on that subject. In the greatest number, the membrane of both nostrils was diseased; in almost all, the lungs were filled with tubercles, either in a hard or suppurated condition. I have annexed thirteen cases, the subjects of which had, at the same time, the tuberculous and schirrous affections combined in the nasal membrane; and eighteen, in which glanders was complicated with farcy.

"In my opinion, then, glanders ought to be: vicwed as a specific affection; and not considered as an un- 
toward termination of strangles, bastard-strangles, grease, and farcy. I would rather admit that all these diseases are different forms assumed by the tuberculous affection.-And then, bastard-strangles, malignant strangles, specific opthalmia, and grease, would be nothing more, in my mind, than effects of the tuberculous affection.

"I have insinuated that the progress of this affection is very tardy; that the tubercles seem not to degenerate until the organic tissues have undergone almost an entire renovation. It would be curious to observe if these animals sink four, six, or seven years after the development of tubercles*. I have remarked, that what gives rise to the tuberculous affection, has a singular influence upon nutrition, and consequently upon the vital action, these phenomena being mutually dependent. But all these operations elude our observation; we must be content to examine the results, and investigate how deranged particular functions affect the economy of general life, and influence the generative operations.

"Perhaps these considerations will show the difficulty of curing such maladies, and engage us in the employment of preservative means, which are greatly more interesting and serviceable; since we might, by a judicious selection of mares and stallions, breed a race of horses insusceptible of them.

\section{"THIRD DIVISION.}

"Considerations on the Acute Glanders.

"GLAN DERS called acute is a disease of another order.

* "I think I have received evidence that animals so affected from hereditary causes rarely survive the seventh year." 
We must not confound it with the tuberculous affection: it has rather some analogies with the typhus of horned cattle, or with the general epizootics, which, at different times, have ravaged France and Europe. We may also discover some affinity between glanders named acute and the angina and peripneumony called gangrenous. All I am desirous of impressing, is, that this disease cannot be considered as glanders; the nature, symptoms, lesions, and means employed in one and in the other, all conspire to distinguish them. Another reason for adopting a distinction would be, were it proved, that a horse once cured is not liable to be affected a second time. And if observation should show that the acute glanders is highly contagious; this and the latter circumstance would approximate it to the rot and typhus in horned cattle."

From several fatal cases, reported with great accuracy, M. DUPUX sums up his more important observations after death, as follows.

"Muscular System. Muscles blanched, fibres softened, pale-colored along the back, and upon the shoulders and withers; their interjacent cellular membrane infiltrated with a yellow serocity or distended with an aeriform fluid. Those of the withers appeared as if they had been scorched.

"Mucous System. The mucous membrane of the trachea, as well as that of the bronchiæ, violet-hued; the internal membrane of the aorta, red, infiltrated, thickened, and easily lacerable, as was also its fibrous tunic. The internal membranes of the crural arteries and carotids, the venæ cavæ and diaphragmatics, were equally reddened. These vessels were full of blood, black, and perfectly liquid, frothy and spumous, as if it had been 
beaten up with so much air; the blood contained in the heart has had the sime observation made upon it. The tissue of the heart was quite flabby, softened, pale, blanched; nevertheless, blood was found extravasated among its fibres, especially upon the internal surface, there being numerous ecchvmoses within both the ventricles.

"The parenchyma of the lungs appeared violet-hued and softened; air was found within the cellular tissue connecting the lobules. This remark is important. How was this gas produced? The same pulmonary emphysema is observed in the typhus of horned cattle. Has the disease in question any analogy to typhus? This question merits our serious attention, and especially when we remember that a reddish sanies abounds in the cellular tissue of the affected regions. This yellowish sanies had become concrete and firm upon the withers, where the spines had grown carious from the alteration within the cavity of the abdomen. The mesenterics were red at their origin.

"The brain was pale and softer than ordinary; the plexus choroides of the cerebrum and cerebellum, yellowish and infiltrated; the pituary gland alike discolored, which formed a contrast with the violet hue of other parts: the sinuses of the bones of the head have also been discovered full of this red serocity.

"The sanious matter obtained from these swellings, introduced under the skin of the neck of a horse in health, produced a similar affection, of which the animal died on the fifth day. The symptoms and lesions observed, manifested no differences of importance. I have repeated these experiments on several other horses, and also on sheep. I have uniformly obtained the same. 
results; whilst a dog who licked and swallowed the: matter, escaped infection." The

\section{"FOURTH DIVISION,"}

Treating of the "Tuberculous Afjection of Domestic Inimuls, compured with thut of the Horse," I shall pass over, and proceed to the

\section{"FIFTH DIVISION.}

"Treatment.

"Il veterinary pathology has for its object the detection, description, classification, and treatment of diseases according to the divers indications shown by them, it will be agreed that a crowd of circumstances conspire to embarrass even the best informed practitioner, which claim from him no ordinary sagacity to enable him to institute a judicious method of cure.-The effects of this destructive disease are sometimes manifested by tubercles upon the exterior of the body : these morbid productions are readily dispersed, especially when their development commences. But such cures are rarely complete; the tubercles very often become reproduced. This relapse of a malady believed to have been cured, manifests extreme obstinacy; it resists means holding out to all appearances the most flattering prospects. It is also observable, that the remedies which prove successful in one case, will often have the very contrary effects in another : this is owing to the variation in the virulence of the disease, and to the greater number of tissues affected, as the lymphatic glands, the mucous membrane of the nose, the pulmonary tissue. The veterinarian therefore should endeavour to frame a system of therapeutics capable of cm- 
bracing every indication, and cast away the specific remedies offered to us with such boasted assurance, which, so far from being beneficial, are very often injurious."

After having laid before us (avec trop de complaisance peut-ĉtre) the different means proposed, by the ancients as well as the moderns, for combatting the tuberculous affection, M. DUPU y continues-"The experience of past ages cannot be set off against me. What advantages do we gain from such experience? What does the mind glean from the perusal of these different veterinary works? I repeat, two leading hypotheses are discoverable (in these works). The one attributes glanders to an acrimony circulating in the blood: to which account they lay all disorders that may supervene upon it. The partisans of this proofless supposition imagine that they will achieve a cure if they can but manage to eliminate or expel this pretended morbific humor; to the fulfilment of which grand desideratum, every remedy employed is directed. According to the second supposition, glanders is viewed as a local affection, attacking the mucous membrane of the nose. Those who have adopted this opinion employ fumigations, and trepan the frontal and maxillary sinuses. Moreover, they have invented instruments to conduct these vapors and fumigations into the different windings of the nasal cavities, with the intention of locally affecting and bathing the diseased parts, and in the end of cicatrizing the chancres by vapours charged with medicinal exhalations; but unfortunately, all these means, whose combination appears so happy, have not been followed by the anticipated success. It certainly is surprising to one who has attentively perused the different works on glanders, from the time of 
Aristotle to the present, that he has met with nothing but a multiformity of suppositions, and not one author who has busied himself about the NATURE of this mortal malady. They have all disregarded this important part of patho$\log y . "$

In laying down the treatment, "I shall commence with an exposition of the preservative methods. And these I shall consider in two different points of view. First, I shall inquire if the disease be hereditary, and afterwards examine the simplest and most practicable means within our reach to preserve the species. Secondly, I shall endeavour to decide whether glanders in horses, or tubercular phthisis, can be the product of a contagious miasm.

"It is more prudent and advantageous to prevent a disease than to be compelled to combat one." - The influence of climate is very remarkable on animals, not only in altering their special characters, but as it affects their morbid dispositions. The first is evinced in sheep more particularly, whose breed alters with the country into which they may be transported; and the second is evidenced in negroes and monkeys who are brought over to England, where many of them contract scrophula, a disease there endemic. Such also is the humidity of the climate that the rot is very prevalent there among the sheep, which, on that account, are sent at an early age to the butchery. "This corroborates my view of the subject, and evinces the great influence that cold combined with humidity exercises over the animal economy.

"That person will render an important service to his country, and to rural economy in general, who may 
show by incontestible evidence that these organic diseases are very often hereditary: the veritable preservative would be discovered were this point once established. -I knew a mare whose body on dissection presented every appearance characteristic of glanders: her filly died at the age of $4 \frac{1}{2}$ years of the same tuberculous affection. The other offspring of this mare inherited her particular conformation, and her propensities to bite and kick. Another mare and her foal discovered on being opened the same organic lesions as the preceding animals. I have made the same observation on the examination of a third mare and her foal. These facts were attested by the Professors and Pupils of the Veterinary School at Alfort; so that they combine unquestionable authenticity. The lesions I have been mentioning were too evident and well-marked to admit of the possibility of any serious mistake."-Here follow other similar observations in relation to os, t1 : sheep, and swine, showing that the tuberculous disease in them, which takes other names-la pommelière des vaches, the rot, and the husk, is likewise transmitted from one generation to another. Indeed, says DUPUV, "I have gathered some facts together proving that the specific opthalmia affecting the horses of certain parts of France, is very often likewise the product of an analogous cause.- M. CoRvisa RT tells us, that from the defective conformation which we derive from our parents, springs the germ of every species of organic disease. I am thoroughly convinced, says this author, that hereditary influence is more powerful and extensive than physicians of the present day have yet conceptions of. I firmly believe that a rery great propor- 
won of diseases, especially of those that resist the efforts of art, arise from causes which, whether they be organic or humoral, are hereditary, and consequently insurmountable. Take for example phthisis pulmonalis.

"These considerations to us are of the greatest moment, since we have it in our power, by coupling and crossing well-known breeds, to lessen the number of animals predisposed to these diseases. Acting up to such ideas, our line of conduct is marked out. We must banish from establishments designed to improve the breed, such animals as show any signs of tuberculous disease or any analogous affection. Above all, no stallion should be allowed to remain in a wet or cold situation, in consequence of the evils likely to result therefrom. - I quit with regret so interesting a subject, one which I propose one day or another to return to again. These preservative measures, if they were generally adopted, might at least afford us consolation for the unsuccessfulness of the different modes of treatment hitherto employed.

"The question of hereditableness has not yet been viewed in the light of positive physiology; it is to be presumed that the reader will readily receive the conclusions I have laid down. But will this be the case with the question I am now going to agitate?"”

The Professor follows this remark with several detailed observations and experiments, received or extracted from some of the most respectable veterinary authorities in France, which go to disprove the opinions, in favor of which the public are so prejudiced, of glanders being a contagious disease. In the course of these relations, it is said, that in regiments of cavalry, to preserve consistency, when the appointments of a horse, 
sacrificed for having glanders, are destroyed, the clothes of the man who looked after him should also share the same fate, and even the stable itself, adds DUPUY, be burnt, since boiling water is said not to annihilate the poison, and the lime which is spread over the surface of the stall, soon falls or breaks off again: this would have been reasonable enough at the time that VITET counselled all the sovereigns in Europe to have slaughtered on the same day every glandered and suspicious subject in their respective dominions, in order to exterminate the disease altogether. In closing this part of his subject, M. DUPUY observes-" "What I have just related is the bare truth, resulting from observation and experience, the two principal tests we possess for proving these points with accuracy and precision. I trust I have submitted my evidence with all possible circumspection; I declare that I have considered the subject medically, and not in accordance with the views of the administrative police. It has been my wish to combat with prejudices. It will be seen that my object has been utility. Have I fulfilled this object without intentional offence to any person whatever? The reader is to decide; and I trust that the essay will meet with his indulgence.

\section{" General Observations relative to Glanders.}

"From my own experience and that of some of my colleagues, it would appear that glanders is rare in very cold countries, and absolutely unknown in hot climates. From and throughout Poland to about the middle of France, glanders is frequently seen; it is not commom on the other side of the Pyrenees; it is unknown in Africa. M. VUILlAUME, my colleague and friend, 
ascertained this lact during his stay at T'unis and 'Tangiers. M. Simon, veterinary surgeon to the king of Portugal, assured him that he had not seen al glandered horse during the thirty years he had been residing in Lisbon.

"Is a cold and humid temperature the only causse? Might not we suspect that castration contributed nuch towards it? I have observed, that this disease morc frequently attacks geldings than stonehorses and mares. We know that in countries wherein glanders appears to be enzootic, it is the custom to submit horses to this enervating operation, one which stamps his form and character with the seal of imbecility and pusillanimousness. We know that the immense tribes of wandering Tartars, the Cossacks upon the borders of the White Sea, the Kirguses, the Bashkirs, and the Nogravans, the Basians, the Karakilpaks, and the Kalmucks, who make as much and perhaps more use of horse: than we do, are unacquainted with any means of depriving them of their organs of reproduction. We are assured that they have no knowledge of the disease under consideration, which, if it be true, will confirm what I have just been advancing. In Spain, Barbary, Egypt, and Arabia, all the horses are entire.

"We observe that glanders less frequently affects horses of small stature, those of slender make, but muscular and high-couraged, than large, coarse, phlegmatic subjects. It would appear that a certain period of age is favorable to its development. I have nothing very positive to offer on this point; so far as my observations go, they show that among forty glandered horses there werc but two under five years old, and but five above twelve. 
"Since the publication of the interesting work of $\mathbf{M}$. Goonv:, Jun. we have remarked that out of eleven cases of glanders there were but two affected in the right nostril: seven discharged from the left, and two from both sides.

"Most of the authors who have written on glanders have servilely copied one another. They have thrown together symptoms to characterize it which belong to a host of other disorders. I can reckon upwards of sixty causes of glanders enumerated by them. Among'so many, they were certain almost of naming the real one." - Among the causes enumerated, are - "damaged provender; over-fatigue; the infuence of cold and humidity; low, wet, dark habitations, infested with currents of air charged with effluvia from the animal matter's uccumulaled about the door and windows of stables, the utmosphere of which is not sufficiently often renewed, and which are situated by the side of rivers, rampart walls, garrison towns, \&c.

\section{"Conclusions.}

" $W_{E}$ learn from experience that certain horses live a long time affected with glanders, while others sink shortly after the appearance of the primary symptoms. It would be important, as regard therapeutics, to discover the cause of these remarkable differences in the progress of the disease."

It has been shown in the first division, how difficult or impossible it is to detect the origin of a disease which in its incipient stage does not sensibly disturb the functions of the organs wherein it is seated. "It is at this period rather a morbific commotion, (un travail morbitique, ) a predisposing cause, than a real malady." 
In the second period, the disease perhaps does not appear to the owner serious enough to submit the animal to treatment, even should we be able to detect it, or distinguish it from catarrh or pnemunonia, and vice versut, which we are not always certain of doing.

"It is the duty of the veterinary surgeon to take steps to remove the predisposing causes, or to resist the pending attack, and treat the disease as soon as it makes its appearance. Siow, the exciting causes of tubercles or hydatids, I have stiated to be, cold, humidity, super-aqueous aliments, stables situate low, illrentilated, wanting light, and placed over drains, near terrass-walls, ramparts, rivers, \&c. lastly, the exposure of horses at bivouac, in wet encampments, or their inhabitation of ill-pared stables, whose atmosphere is confined, and vitiated with the efluria from the dung; and where food is insufficient, and that often of bad quality. It is of the first consequence to remove diseased animals away from such deleterious influences before we set about combatting, with suitable measures, the general diathesis to which the disease owes its origin.

"Let us bear in mind, in the treatment, that the tuberculous affection consists in a molecular movement going on in the affected organ, whence springs a morbific production, cudowed with an action opposed to that one by which the organ is nourished in a state of health. It is then this intestine movement that must be rectified in order to make a radical cure. Is this rectification well understood by us? How is it to be carried into effect? What means are we to employ to restore the nutritive action to its ordinary siandard? These indications are extremely difficult to accomplish. 
"To be successful, it is evident that the beneficial influences of regimen and nedicaments must be continued long enough to work a sort of metamorphosis in the affected parts. In fuct, what can one expect from remedies which operate but in a subitaneous manner? They can only excite a superficial action, one that can have no effect in altering the composition of the organ. This excitement might even do harm; a result always to be expected when prescriptions are hazarded, without having first learnt from experience the changes it is expedient to bring about to arrest the progress of these affections.

"The treatment should be distinguished into what relates to regimen or dietetic means, and into medical treatment. The regimen should be corroborative; the aliments of good quality; the infirmary in a dry airy situation, avoiding low wet grounds, and be well ventilated; the work or exercise moderate, and during fine weather; dressing, hand-rubbing, and wisping are useful, and supply the want of exercise when that cannot be given. These means have a powerful influence on the constitution of subjects of the tuberculous affection; they retard its progress; the disease seems to be arrested, though it muns on enfeebled for several years.

"Could we not introduce into the regimen of these animals vegetable substances containing a large proportion of azote, such as wheat and gluten? And why not add to this, vinous panadas and broths?" ViceD'Azir assures us, that he has seen a great number of beasts recover (from epizootics) after having taken meat soups. In Sweden, according to the same author, bread is made from oatmeal, by which it is found that 
horses may be kept at one-half the ordinary expense.To these matters I propose some day to return.

"Among the great number of medicaments which have been recommended for this affection, the best, which are far from succeeding under a multiplicity of circumstances, are tonics, such as gentian, elecampane, tansy, aromatic powders, quinquina, hemlock, digitalis, and the phellandrium aquaticum. Among the mineral substances, sal ammoniac, carbonate of ammonia, ammonia, kermes mineral, emetic antimony, corrosive sublimate, mercurius dulcis, the sulphurets of mercury - cinnabar and ethiops mineral, sulphur, and sulphuret of soda.

"Aloes, ammoniacum, assafœetida, resin, nitre; revulsive means, such as setons, blisters, firing the sides to diminish the pulmonary inflammation often complicated with this affection.

"Let not these medicaments, taken singly, be looked upon as specifics; (we have no such thing;) but as agents capable of producing beneficial effects in the hands of scientific practitioners, when judiciously combined and skilfully exhibited. I might have affixed the doses and prescribed such formulæ as I have found most successful ; but, as I am persuaded that veterinarians stand in no need of this, I thought it superfluous to insert them, thereby depriving empirics of arms they might use injuriously to farmers, and acting up to the laws in force which are in opposition to the institution of any treatment likely to prove successful in combatting the various complications of which the tuberculous affection is susceptible."

Having at length arrived at the end of M. DuPUY's profound, accurate, and scientific researches into the 
origin and nature of glanders, by which we are led into an entirely new channel of investigation, and therein considerably enlightened in our progress, I shall (purposely refraining from offering anything of my own on the subject until such opinions as I may entertain shall have been further matured) bring this series of lectures to a conclusion. I may now with DuPUY say, "Ici se termine la tâche que nous nous sommes imposée. Elle auroit exigé peut-être de notre part et plus d'expérience et plus d'instruction." If I have evinced but accuracy in my anatomical descriptions, correctness in my physiological views, and faithfulness in reporting what is known in veterinary medicine and surgery, I have ac. complished my original design. I am not aware of having made more than one very important omission; and that is the subject of the ear: though a part, as far as my observations have gone, in the horse exempt from disease, yet it is one entitled to a place in a regular course of anatomy. I hope, one day or another, to take an opportunity of supplying the deficiency. The foot has been past over by design. I considered that what new or original matter I might have found to offer on the subject, would hardly warrant me to break up that ground afresh which has already been so extensively cultivated through the labors of Messrs. Coleman, CLARKE, and GoODWIN; and therefore I have refrained from making this important part an unprofitable addition to the present series of lectures.

'THE END. 




